\title{
Photocatalytic dehydroxymethylative arylation by synergistic cerium and nickel catalysis
}

\author{
Yuegang Chen ${ }^{\dagger}$, Xin Wang ${ }^{\dagger}, \mathrm{Xu} \mathrm{He}^{\dagger}$, Qing $\mathrm{An}^{\dagger}$ and Zhiwei Zuo ${ }^{\ddagger \star}$ \\ ${ }^{\dagger}$ School of Physical Science and Technology, ShanghaiTech University, Shanghai 201210, \\ China
}

$¥$ State Key Laboratory of Organometallic Chemistry, Shanghai Institute of Organic Chemistry,

Chinese Academy of Sciences, Shanghai 200032, China 


\section{Contents}

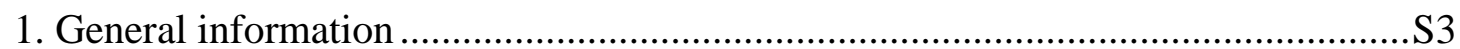

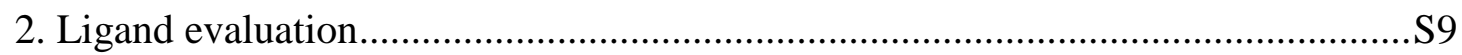

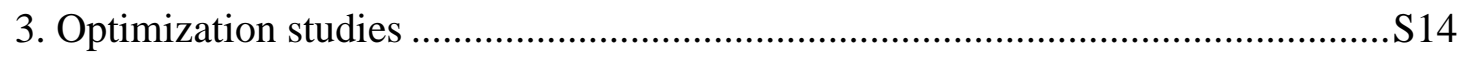

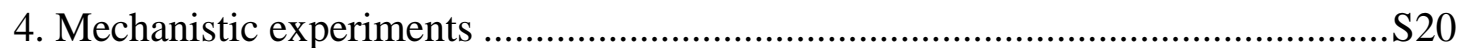

5. Experimental procedures and spectral characterization....................................S29

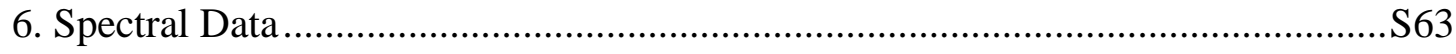

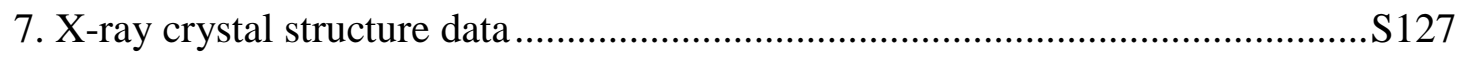

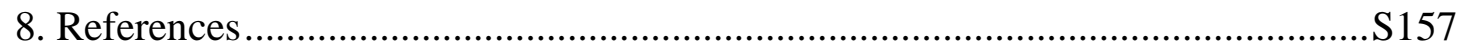




\section{General information}

\subsection{Materials and Methods}

All commercial reagents and solvents were used as received unless otherwise indicated. $\mathrm{CeCl}_{3}$ was purchased from Alfa Aesar. 9,10-diphenylanthracene (DPA) was purchased from TCI. Dimethyl sulfoxide (DMSO) was purchased from Energy. Other reagents were purchased from Aldrich, TCI, Adamas, Energy and $\mathrm{J} \& \mathrm{~K}$ chemical. $\mathrm{CeCl}_{3}$ were stored and weighed out in glovebox. Acetonitrile was purified by a Vigor solvent purification system.

Gas chromatography (GC) was carried out on an Agilent 7890B system using HP5 column (5\% phenyl - 95\% methyl polysiloxane; $30 \mathrm{~m}, 0.32 \mathrm{~mm}$ ID, $0.25 \mu \mathrm{m}$ df). Yields and selectivity ratios were determined by calibrated GC-FID analysis after the addition of anisole as internal standard.

NMR spectra were obtained from Bruker Avance III HD 500. ${ }^{1} \mathrm{H}$ NMR spectra were recorded at $500 \mathrm{MHz},{ }^{19} \mathrm{~F} \mathrm{NMR}$ were recorded at $470 \mathrm{MHz}$, and ${ }^{13} \mathrm{C}$ NMR spectra were recorded at $125 \mathrm{MHz} .{ }^{1} \mathrm{H}$ NMR chemical shifts were determined relative to the signal of the residual protonated solvent: $\mathrm{CDCl}_{3} \delta 7.26 \mathrm{ppm}$ and DMSO- $d_{6} \delta 2.50 \mathrm{ppm} .{ }^{13} \mathrm{C}$ NMR chemical shifts were determined relative to $\mathrm{CDCl}_{3}$ at $\delta 77.0 \mathrm{ppm}$ and DMSO- $d_{6}$ $\delta 39.52 \mathrm{ppm}$. Data for ${ }^{1} \mathrm{H}$ NMR are reported as follows: chemical shift $(\delta \mathrm{ppm})$, multiplicity $(\mathrm{s}=$ singlet, $\mathrm{d}=$ doublet, $\mathrm{t}=$ triplet, $\mathrm{m}=$ multiplet, $\mathrm{q}=$ quartet, $\mathrm{br}=$ broad $)$, integration, coupling constant $(\mathrm{Hz}) .{ }^{13} \mathrm{C}$ NMR spectra were reported as chemical shifts in ppm and multiplicity where appropriate. ${ }^{19} \mathrm{~F}$ NMR spectra were reported as chemical shifts in ppm. 
High Resolution Mass spectra were obtained from Thermo Fisher Q-Exactive Highresolution MS.

Chromatographic purification of products was accomplished by flash chromatography using silica gel. Thin-layer chromatography (TLC) was performed on Silicycle $250 \mathrm{~mm}$ silica gel F-254 plates. 


\subsection{Photoredox setup for batch reactions}

Photoredox reactions were performed using a customized photoreactor (Figure S1) with maximum capacity of 24 parallel reactions. The reaction vials were irradiated by the LED chips underneath. The reaction vials and LED chips were cooled by the aluminum plate with continuous water flow, maintaining ambient temperature.
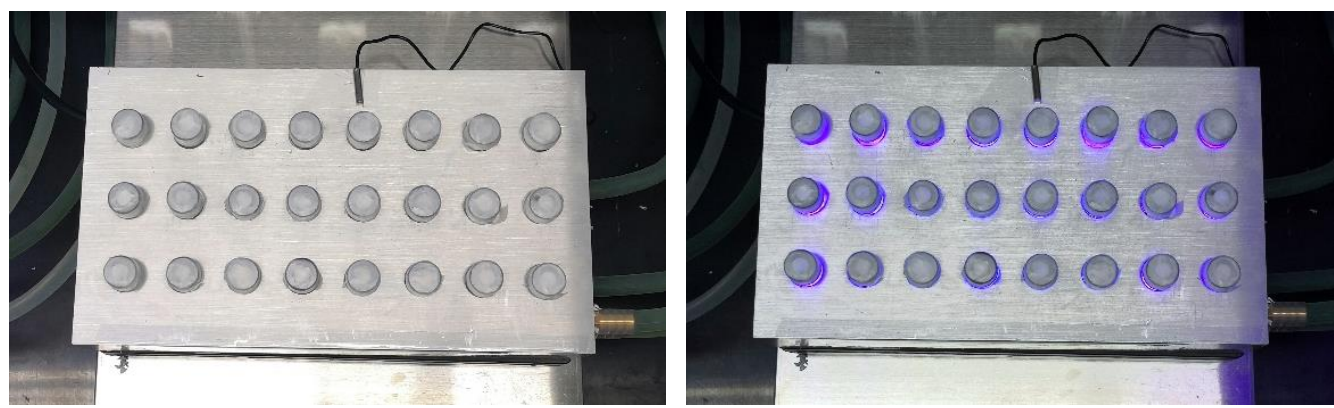

Figure S1. Details for the photoredox setup. 


\subsection{Automated screening set assembly using Chemspeed SWING ISYNTH platform}

A customized high-throughput experimentation platform (Chemspeed SWING ISYNTH, Figure S2) was used to evaluate a large collection of ligands. When the front hatch of the chamber is closed, nitrogen is allowed to flow inside the chamber through inert gas distribution tubing around the lower perimeter of the deck, and then an inert atmosphere is obtained.

The Chemspeed SWING ISYNTH platform uses a GDU-P fine dosing (more than $10 \mathrm{mg}$ ) or GDU-S (less than $10 \mathrm{mg}$ ) that incorporates an internal balance to weigh and transfer the desired amount of chemical. Chemspeed AutoSuite was used to set all the required variables including quantities and screening plate positions to transfer the targeted amount of substance to the destination vials. For GDU-P fine dosing, the extruder screw rotates within the cap of the solid dispensing bottle to distribute the solid material. The internal balance monitors the amount of solid dispensed throughout the dispensing process. For GDU-S, solid material is picked up by sticking a transfer tip (SWIN) into source vial and then measured by the balance. When the substance is released into the target vial, the (now empty) tip is weighed again to determine exact amount of substance added. After the targeted mass is transferred, the used SWIN tip is discarded to a waste position and a new tip is picked up.

With the assistance of Chemspeed SWING ISYNTH platform, a typical 48 reaction condition screening set required more than 150 solid materials including catalysts, ligands and bases was completed in 1 hour. 


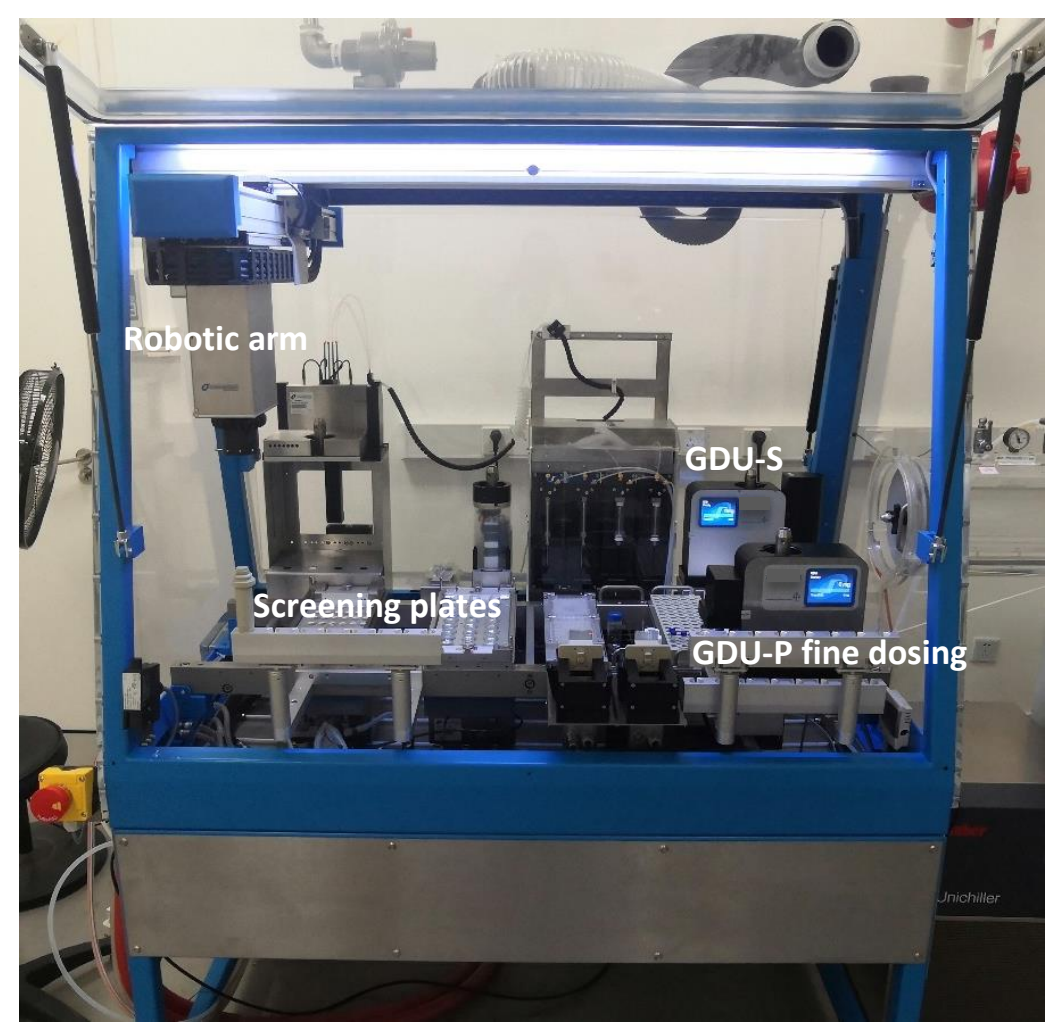

Figure S2. Chemspeed SWING ISYNTH system.

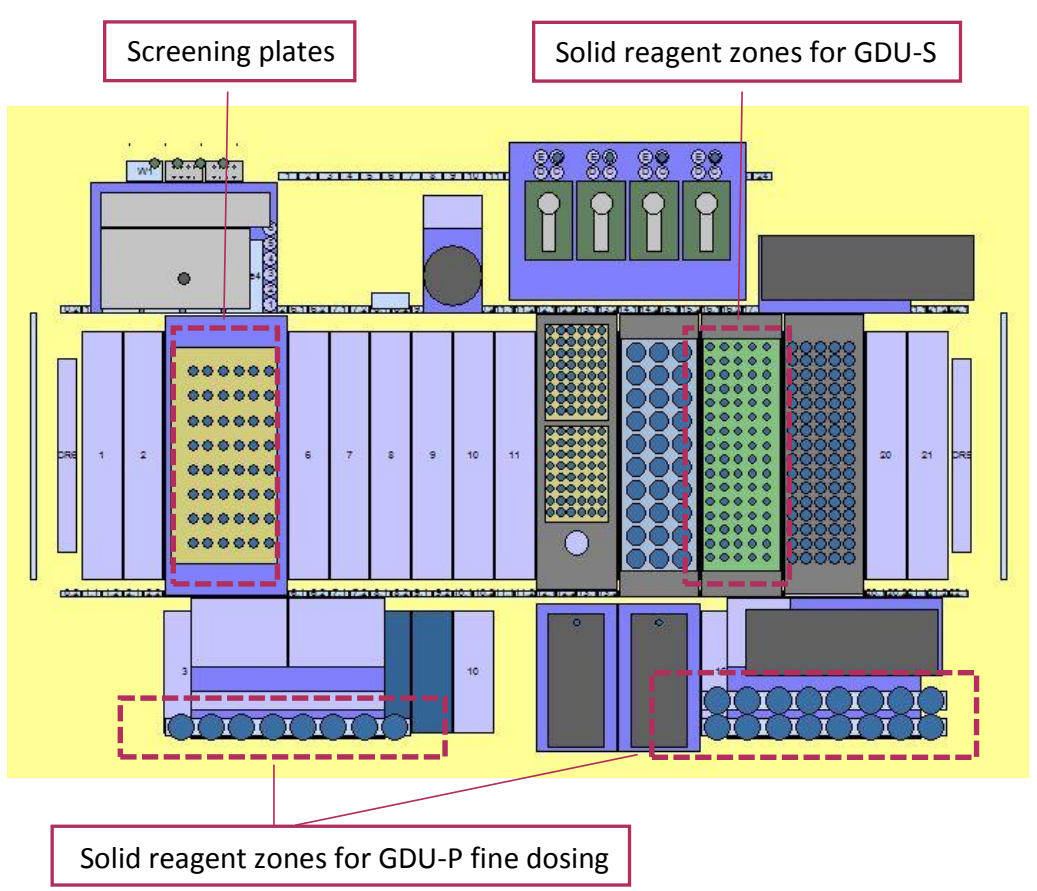

Figure S3. Screenshot of AutoSuite control software. 


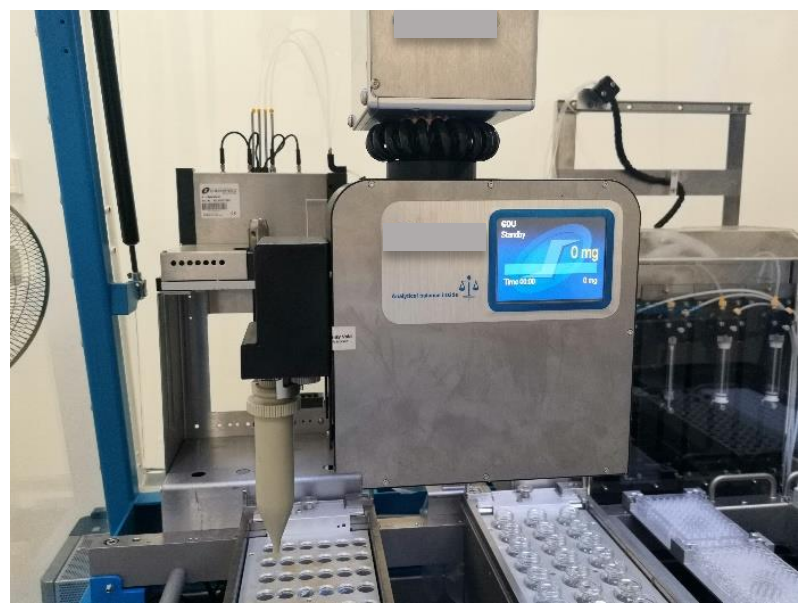

Figure S4. Chemspeed SWING ISYNTH GDU-P fine dosing.

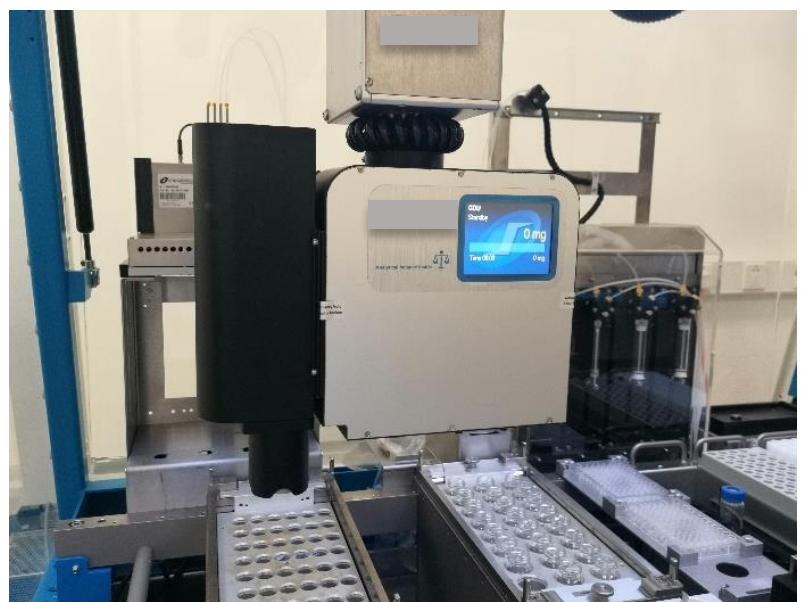

Figure S5. Chemspeed SWING ISYNTH GDU-S.

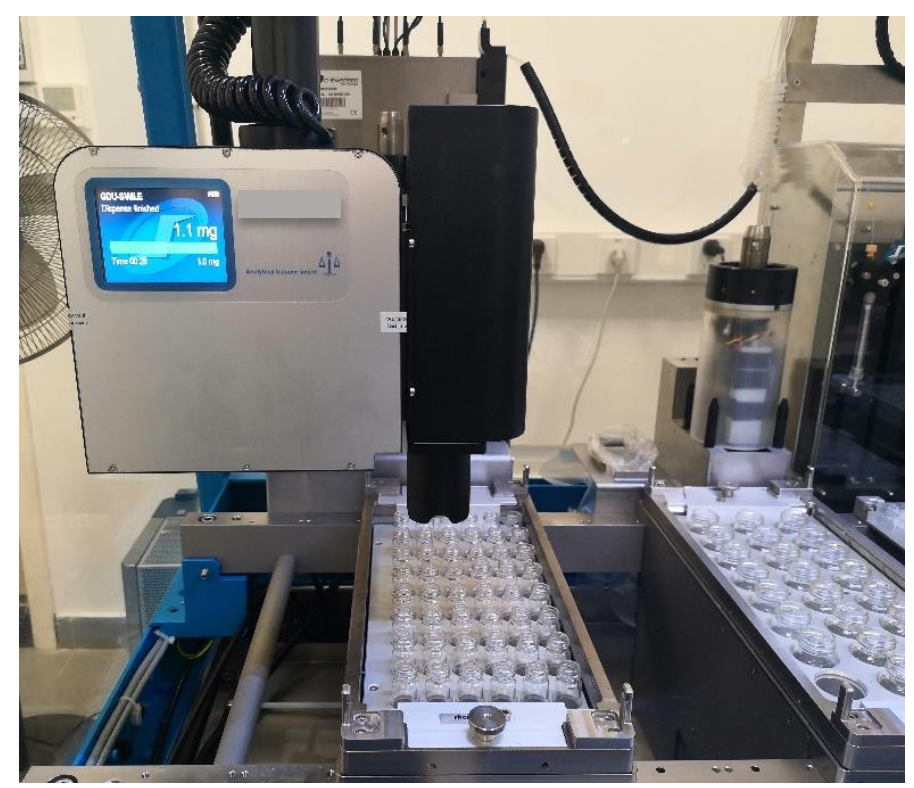

Figure S6. Chemspeed SWING ISYNTH GDU-S dispensing solid materials to the designated positions on screening plates. 


\section{Ligand evaluation}

\subsection{General procedure for automated high-throughput screenings}

In a typical high-throughput experiment, reagents were transferred into $8 \mathrm{~mL}$ vials under the control of Chemspeed AutoSuite. After the reactions were assembled, the reaction vials were placed in a 48 well metal block which was placed on an orbital shaker. A watercooling $400 \mathrm{~nm}$ LED lamp plate matched with the 48-well metal block was then placed directly above the vials and irradiating from the top $(4 \mathrm{~cm}$ distance from the liquid surface, $0.30 \mathrm{~W} \mathrm{~cm}^{-2}$ ).

After 12 hours, internal standard (anisole) was added to each reaction vial using microliter syringe. An aliquot of the reaction mixture was then analyzed by GC-FID.

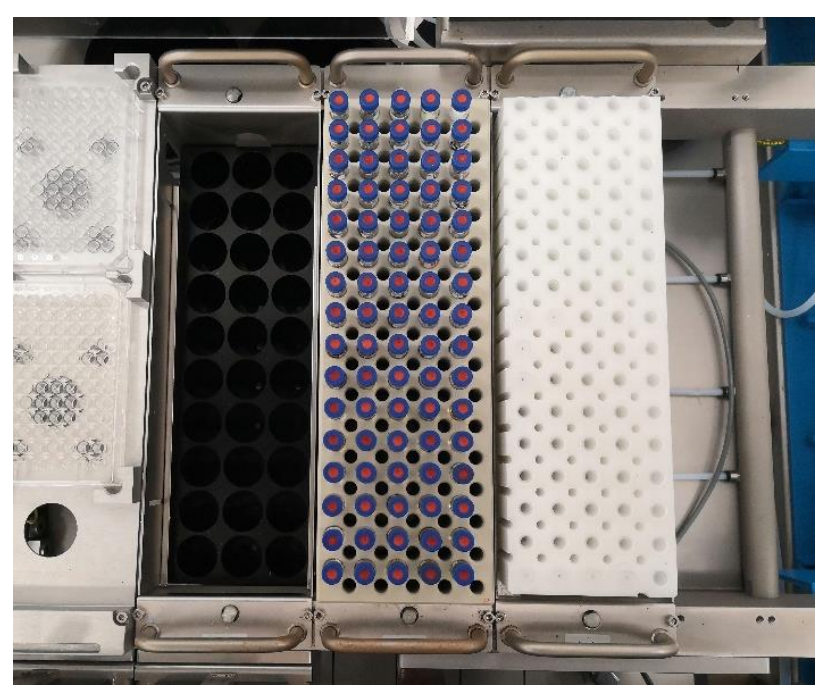

Figure S7. Assembled screening set with ligands. 


\subsection{Evaluation results}

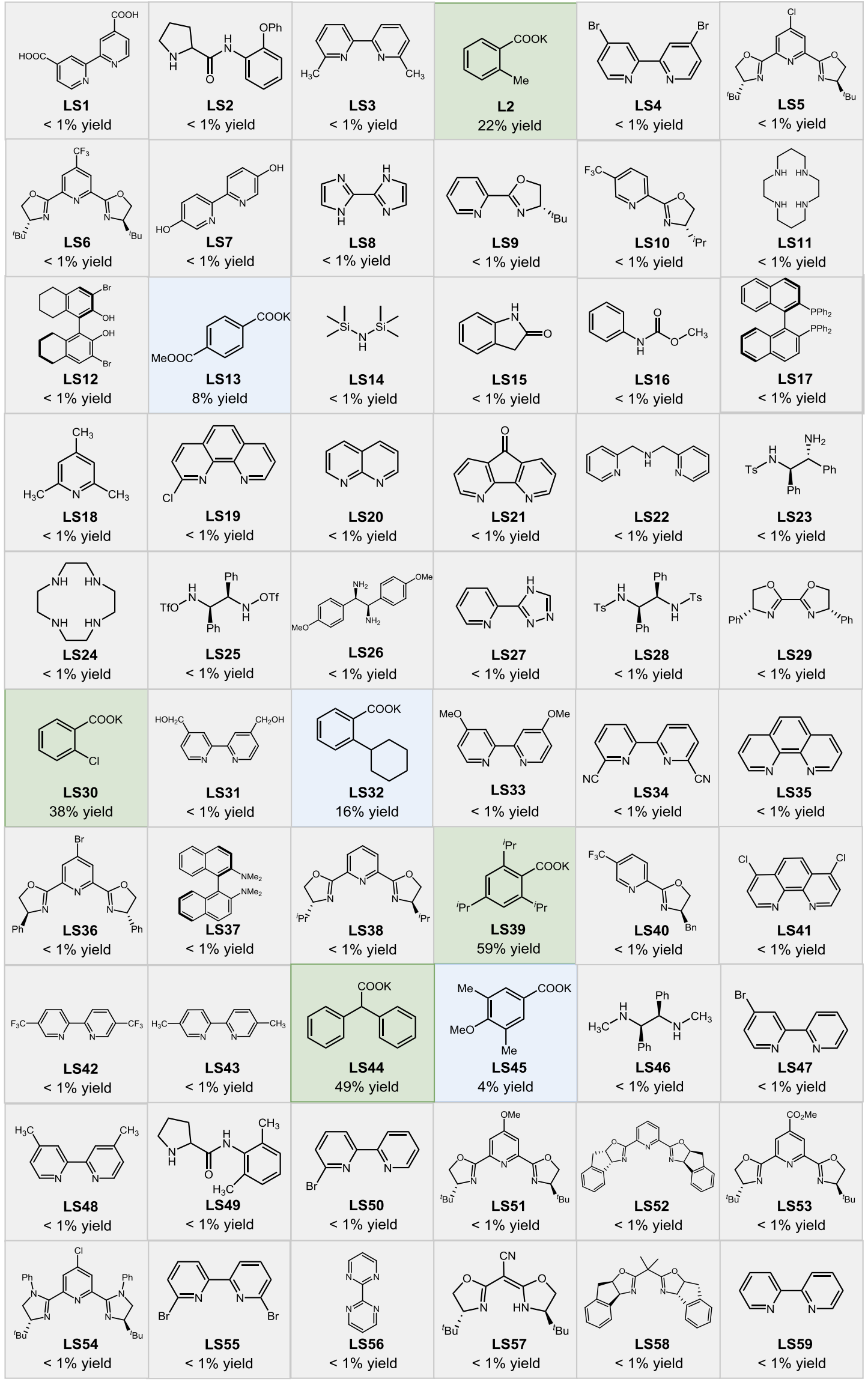




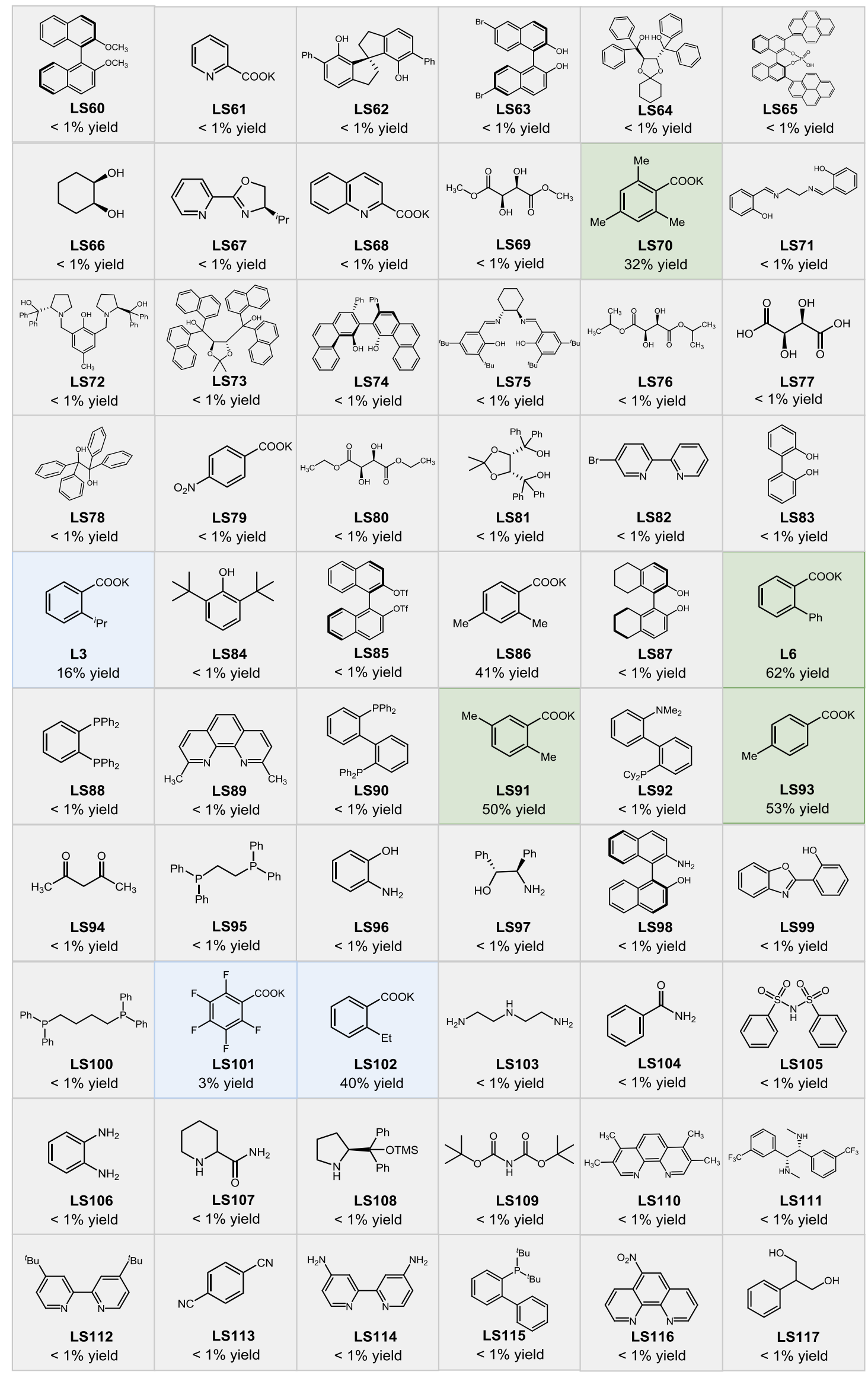




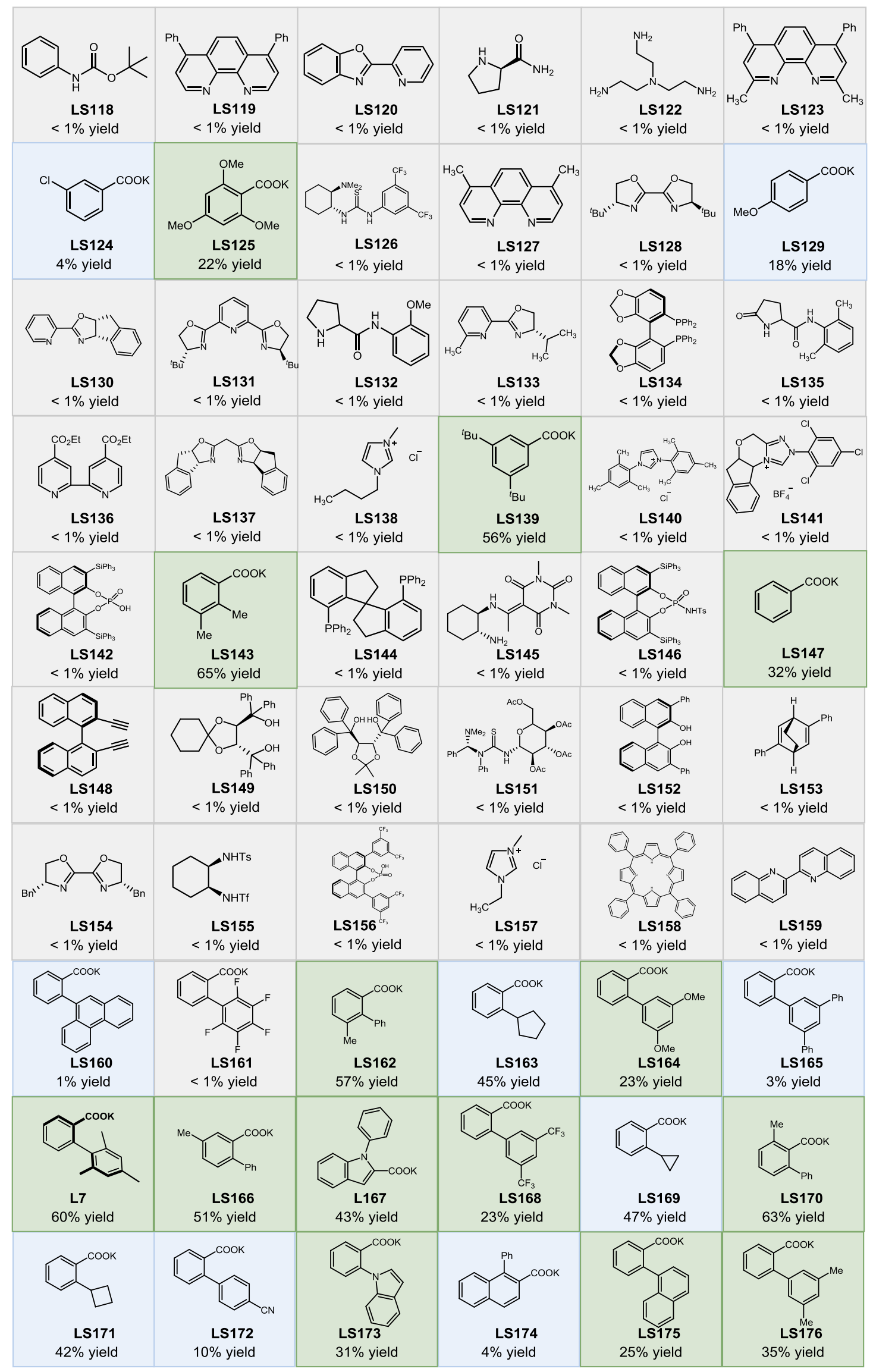




(18)




\section{Optimization studies}

\subsection{Optimization for ligands}

Table S1. Ligand optimization.

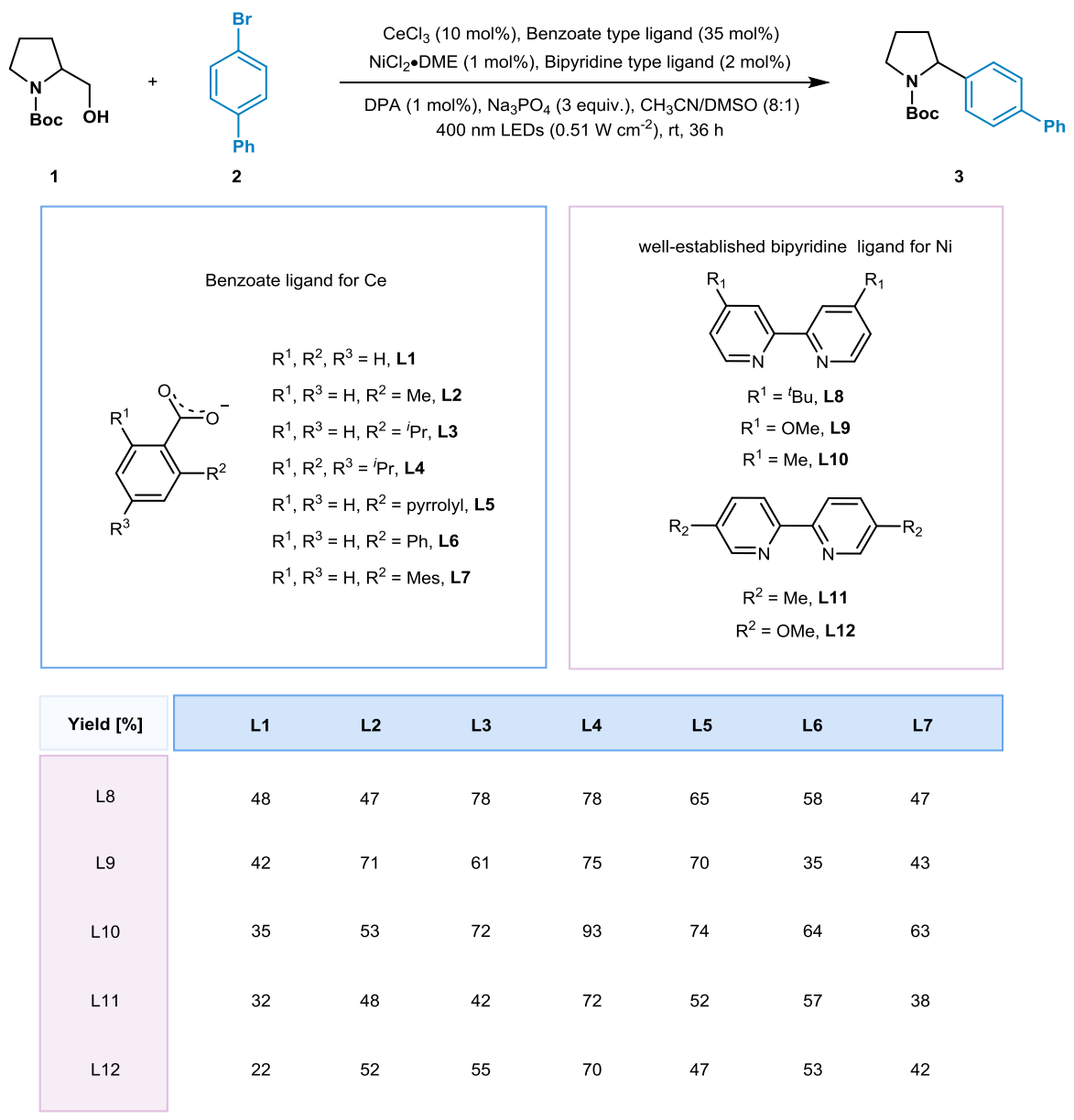




\subsection{Control experiments}

Table S2. Control experiments.

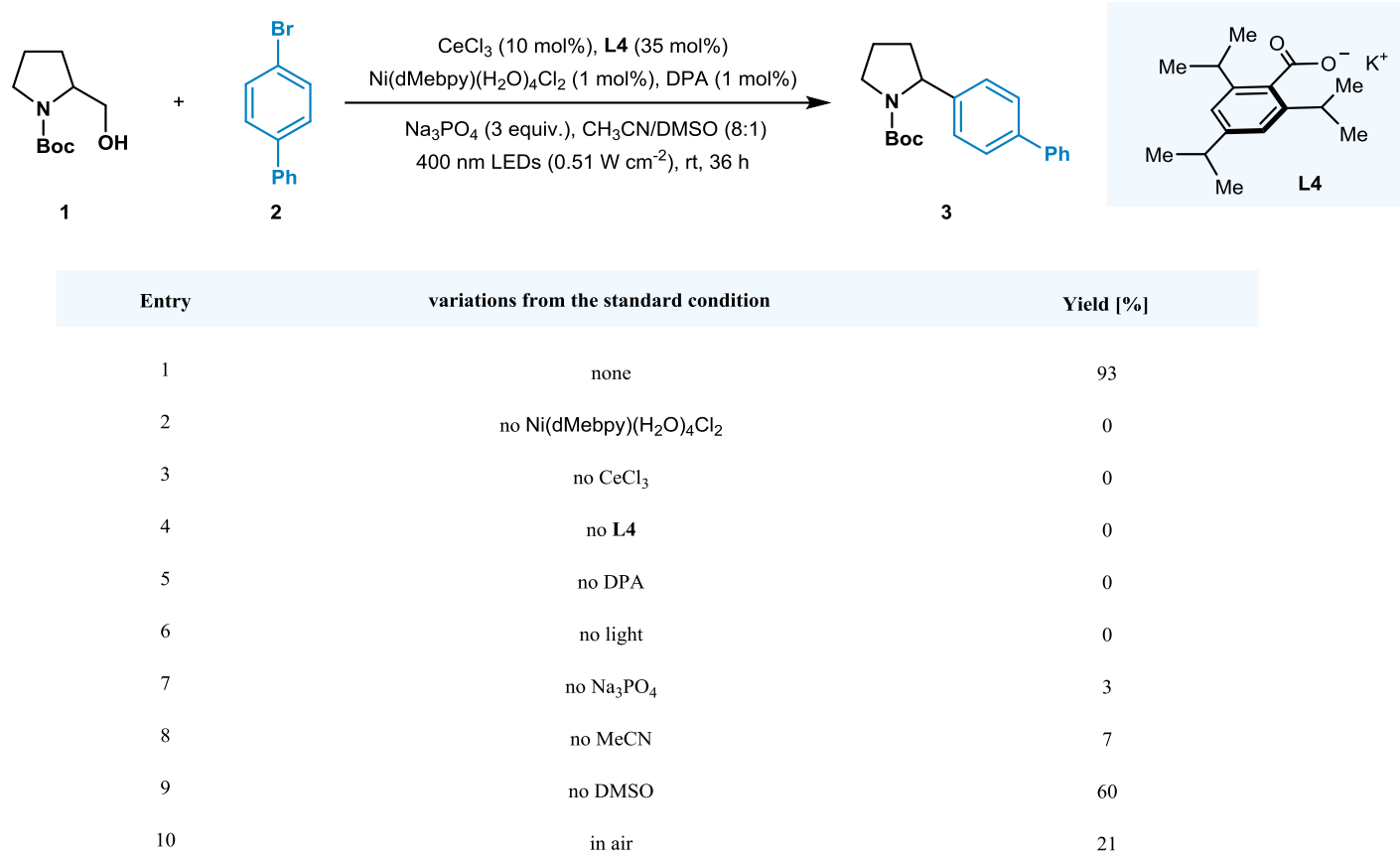




\subsection{Evaluation of metal catalysts}

Table S3. Evaluation of metal catalysts.

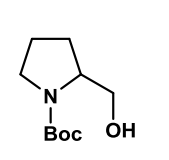<smiles>Pc1ccc(Br)cc1</smiles>

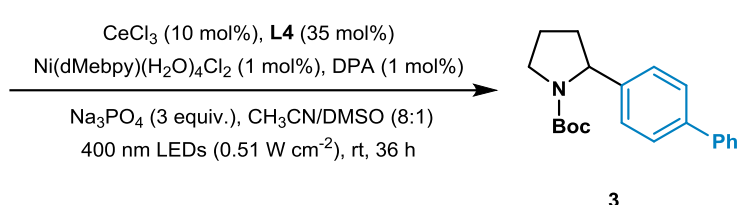

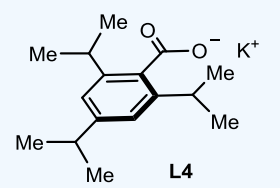

Yield [\%]

variations from the standard condition

none

93

TRIPCOOLi instead of TRIPCOOK $\left(\mathbf{L}_{\mathbf{4}}\right)$

21

TRIPCOONa instead of TRIPCOOK $\left(\mathbf{L}_{\mathbf{4}}\right)$

TRIPCOOH instead of TRIPCOOK $\left(\mathbf{L}_{4}\right)$

$\mathrm{CeBr}_{3}$ instead of $\mathrm{CeCl}_{3}$

$\mathrm{Ce}(\mathrm{OTf})_{3}$ instead of $\mathrm{CeCl}_{3}$

$\mathrm{Ce}(\mathrm{OTf})_{4}$ instead of $\mathrm{CeCl}_{3}$

$\mathrm{Ce}(\mathrm{OAc})_{3} \bullet \mathrm{H}_{2} \mathrm{O}$ instead of $\mathrm{CeCl}_{3}$

$\mathrm{Ce}\left(\mathrm{NO}_{3}\right)_{3} \bullet 6 \mathrm{H}_{2} \mathrm{O}$ instead of $\mathrm{CeCl}_{3}$

$\mathrm{CeI}_{3}$ instead of $\mathrm{CeCl}_{3}$

$\mathrm{Ce}_{2}\left(\mathrm{C}_{2} \mathrm{O}_{4}\right)_{3} \bullet \mathrm{H}_{2} \mathrm{O}$ instead of $\mathrm{CeCl}_{3}$

$\left(n-\mathrm{Bu}_{4} \mathrm{~N}\right)_{2} \mathrm{CeCl}_{6}$ instead of $\mathrm{CeCl}_{3}$
64

53

80

50

73

15

46

2

0

47 


\subsection{Evaluation of benzoate ligands}

Table S4. Evaluation of benzoate ligands.

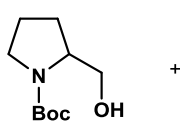<smiles>O=C(O)c1ccccc1</smiles>

L1 $35 \%$ yield<smiles>O=C(O)c1ccccc1-n1cccc1</smiles>

L5, $74 \%$ yield<smiles>COC(=O)c1c(OC)cc(OC)cc1OC</smiles>

LS125, $28 \%$ yield<smiles>Brc1ccc(-c2ccccc2)cc1</smiles>

2

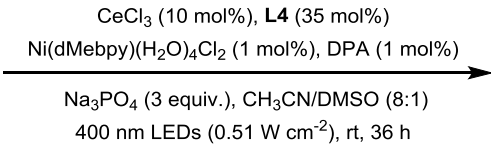

$400 \mathrm{~nm}$ LEDs $\left(0.51 \mathrm{~W} \mathrm{~cm}^{-2}\right)$, rt, $36 \mathrm{~h}$

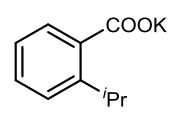

L3, $72 \%$ yield

L2, $53 \%$ yield<smiles>CC(=O)OC(=O)c1ccccc1-c1ccccc1</smiles>

L6, 64\% yield<smiles>CC(C)(C)c1cc(Br)c(C(=O)OCc2ccccc2)c(C(C)(C)C)c1</smiles>

LS193, 10\% yield<smiles>CC(=O)OC(=O)c1ccccc1C</smiles>

L7, $63 \%$ yield<smiles>O=C(O)c1c(O)cc(Cl)cc1Cl</smiles>

LS $180,0 \%$ yield

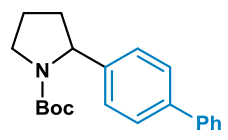<smiles>CCCc1cc(CCC)c(C(=O)OC)c(CCC)c1</smiles>

L4, 93\% yield<smiles>CC(=O)Oc1c(C)cc(C)cc1C</smiles>

LS194, 64\% yield<smiles>CC(=O)c1ccccc1Cl</smiles>

LS195, 33\% yield 


\subsection{Evaluation of benzoate ligand loading}

Table S5. Evaluation of benzoate ligand loading.

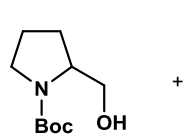<smiles>Pc1ccc(Br)cc1</smiles>

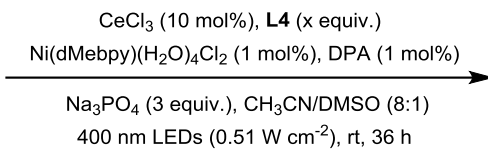

$400 \mathrm{~nm}$ LEDs $\left(0.51 \mathrm{~W} \mathrm{~cm}^{-2}\right), \mathrm{rt}, 36 \mathrm{~h}$

Benzoate ligand loading

0.1 equiv. L4

0.2 equiv. L4

0.3 equiv. L4

0.35 equiv. $L 4$

0.4 equiv. L4

0.5 equiv. $\mathbf{L} 4$

0.6 equiv. $L 4$

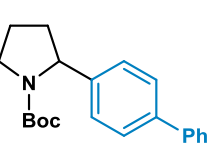

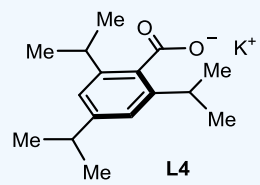

Yield [\%]

4

29

78

93

85

72

62 


\subsection{Recovery of the benzoate ligand}

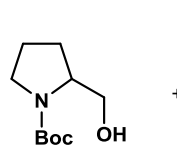<smiles>Pc1ccc(Br)cc1</smiles>

2

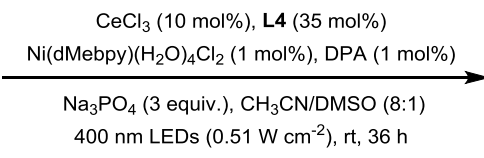

$400 \mathrm{~nm}$ LEDs $\left(0.51 \mathrm{~W} \mathrm{~cm}^{-2}\right), \mathrm{rt}, 36 \mathrm{~h}$

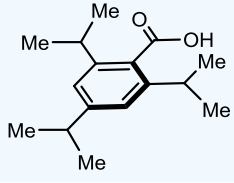

$93 \%$ recovered

Figure S8. Recovery of benzoate ligand. 


\section{Mechanistic experiments}

\subsection{Preparation of cerium complex}

CeL3 (56): A solution of $\mathrm{CeCl}_{3}$ (246.5 mg, $1 \mathrm{mmol}$, 1 equiv.) in water (5 mL) was added to a stirred solution of potassium 2',4',6'-trimethyl-[1,1'-biphenyl]-2-carboxylate (834 $\mathrm{mg}, 3 \mathrm{mmol}, 3$ equiv.) in water $(5 \mathrm{~mL})$. Instantly, a white precipitate was produced. Stirring was continued for a further $12 \mathrm{~h}$, then the solution mixture was filtered. The white solid was washed with $\mathrm{H}_{2} \mathrm{O}$ to yield product $\left(720 \mathrm{mg}, 84 \%\right.$ yield). ${ }^{1,2}{ }^{1} \mathbf{H}$ NMR (500 MHz, DMSO-d $d_{6}$ and CD3OD) $\delta 9.24(\mathrm{~s}, 1 \mathrm{H}), 7.74(\mathrm{~m}, 2 \mathrm{H}), 7.37-7.11(\mathrm{~m}, 1 \mathrm{H})$, 6.79 (s, 2H), 2.16 (m, 9H). ${ }^{13} \mathrm{C}$ NMR (125 MHz, DMSO-d $d_{6}$ and CD CDD $_{3} \delta$ 173.0, 143.8, 141.3, 140.8, 135.7, 135.1, 131.0, 130.9, 130.2, 127.6, 127.2, 20.8, 20.4.

${ }^{1} \mathrm{H}$ NMR $500 \mathrm{MHz}$, DMSO- $d_{6}$ and $\mathrm{CD}_{3} \mathrm{OD}$ :

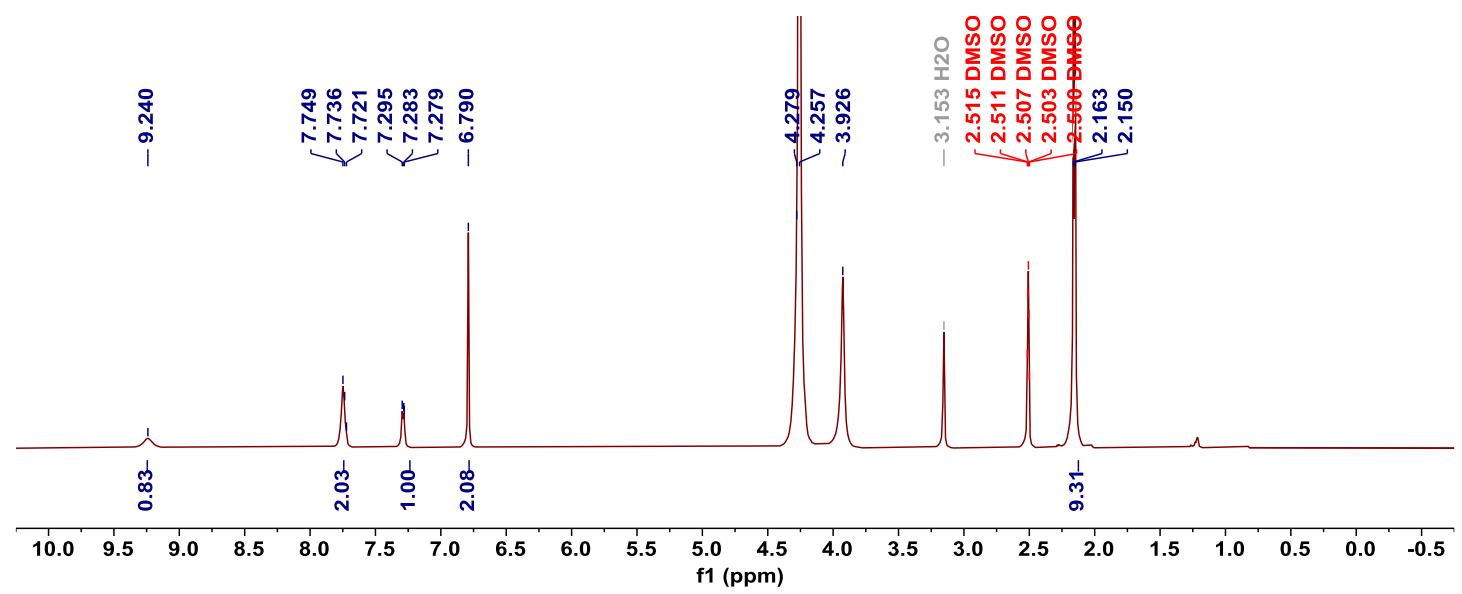

Figure S9. ${ }^{1} \mathrm{H}$ NMR spectrum of the $\mathrm{CeL}_{3}$.

${ }^{13} \mathrm{C}$ NMR $125 \mathrm{MHz}$, DMSO- $d_{6}$ and $\mathrm{CD}_{3} \mathrm{OD}$ :

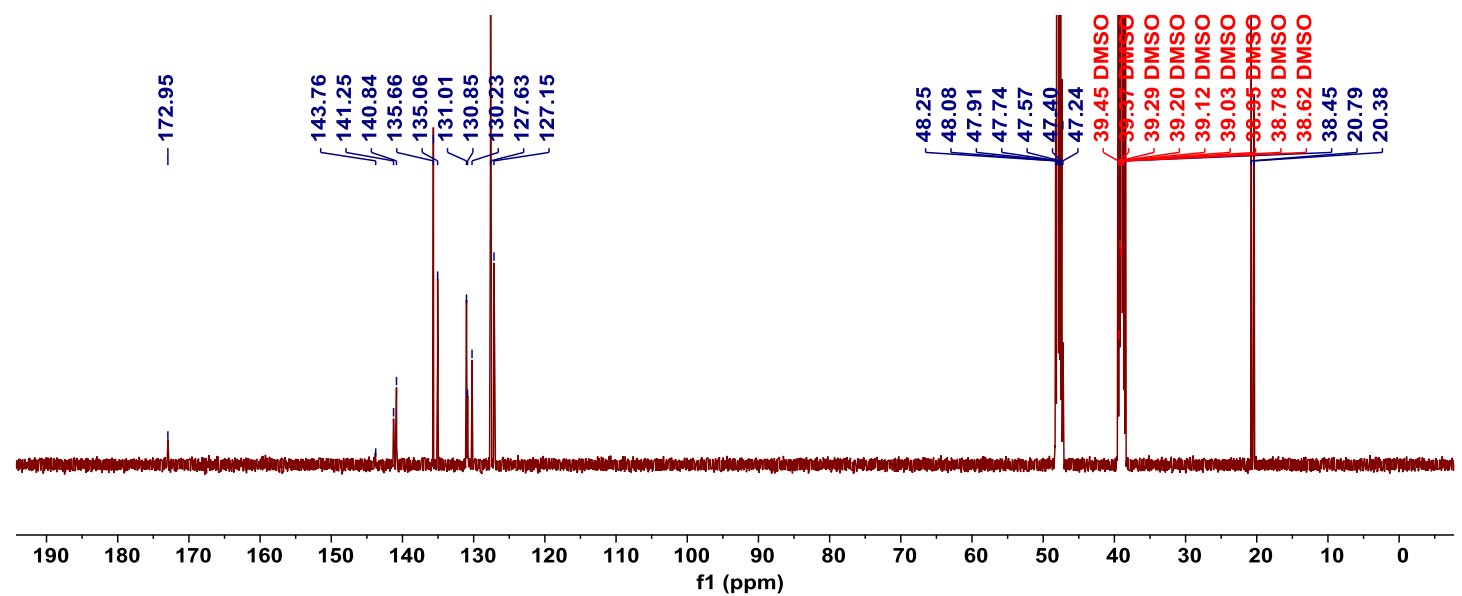

Figure S10. ${ }^{13} \mathrm{C}$ NMR spectrum of the $\mathrm{CeL}_{3}$. 
[Ce(OMe)L3]: A mixture of $\mathrm{CeL}_{3}(171.4 \mathrm{mg}, 0.2 \mathrm{mmol}, 1.0$ equiv.), methanol (128.0 mg, 4.0 mmol, 20.0 equiv.) and $\mathrm{Na}_{3} \mathrm{PO}_{4}$ (163.9 mg, $1.0 \mathrm{mmol}, 10.0$ equiv.) in $\mathrm{CH}_{3} \mathrm{CN} / \mathrm{DMSO}(8: 1,4 \mathrm{~mL})$ was stirred under an nitrogen atmosphere in dark for $2 \mathrm{~h}$. The solution was filtered and diluted to appropriate concentration with $\mathrm{CH}_{3} \mathrm{CN} / \mathrm{DMSO}$ (8:1) for further mechanistic experiments.

$\left[\mathbf{C e}(\mathbf{I V}) \mathrm{L}_{3} \mathrm{Cl}_{3}\right]^{2-:}$ : A mixture of $\left(n-\mathrm{Bu}_{4} \mathrm{~N}\right)_{2} \mathrm{CeCl}_{6}(417 \mathrm{mg}, 0.5 \mathrm{mmol}, 1.0$ equiv. $)$, Potassium 2',4',6'-trimethyl-[1,1'-biphenyl]-2-carboxylate (417 mg, $1.5 \mathrm{mmol}, 3$ equiv.) in $\mathrm{CD}_{3} \mathrm{CN}(10 \mathrm{~mL})$ was stirred under a nitrogen atmosphere in dark for $12 \mathrm{~h}$. The solution was filtered and diluted to appropriate concentration for further mechanistic experiments. (Note: we found that Potassium 2',4',6'-trimethyl-[1,1'-biphenyl]-2carboxylate is slightly soluble in $\mathrm{CD}_{3} \mathrm{CN}$ and the free carboxylic acid is soluble, their ${ }^{1} \mathrm{H}$ NMR spectrum have been attached for comparison). The integration reveals an approximate carboxylate $/ n-\mathrm{Bu}_{4} \mathrm{~N}^{+}$ratio of $3: 2$, suggesting the composition $\left[\mathrm{Ce}(\mathrm{IV}) \mathrm{L}_{3} \mathrm{Cl}_{3}\right]\left(n-\mathrm{Bu}_{4} \mathrm{~N}\right)_{2}$.

${ }^{1} \mathrm{H}$ NMR $500 \mathrm{MHz}, \mathrm{CD}_{3} \mathrm{CN}$ :

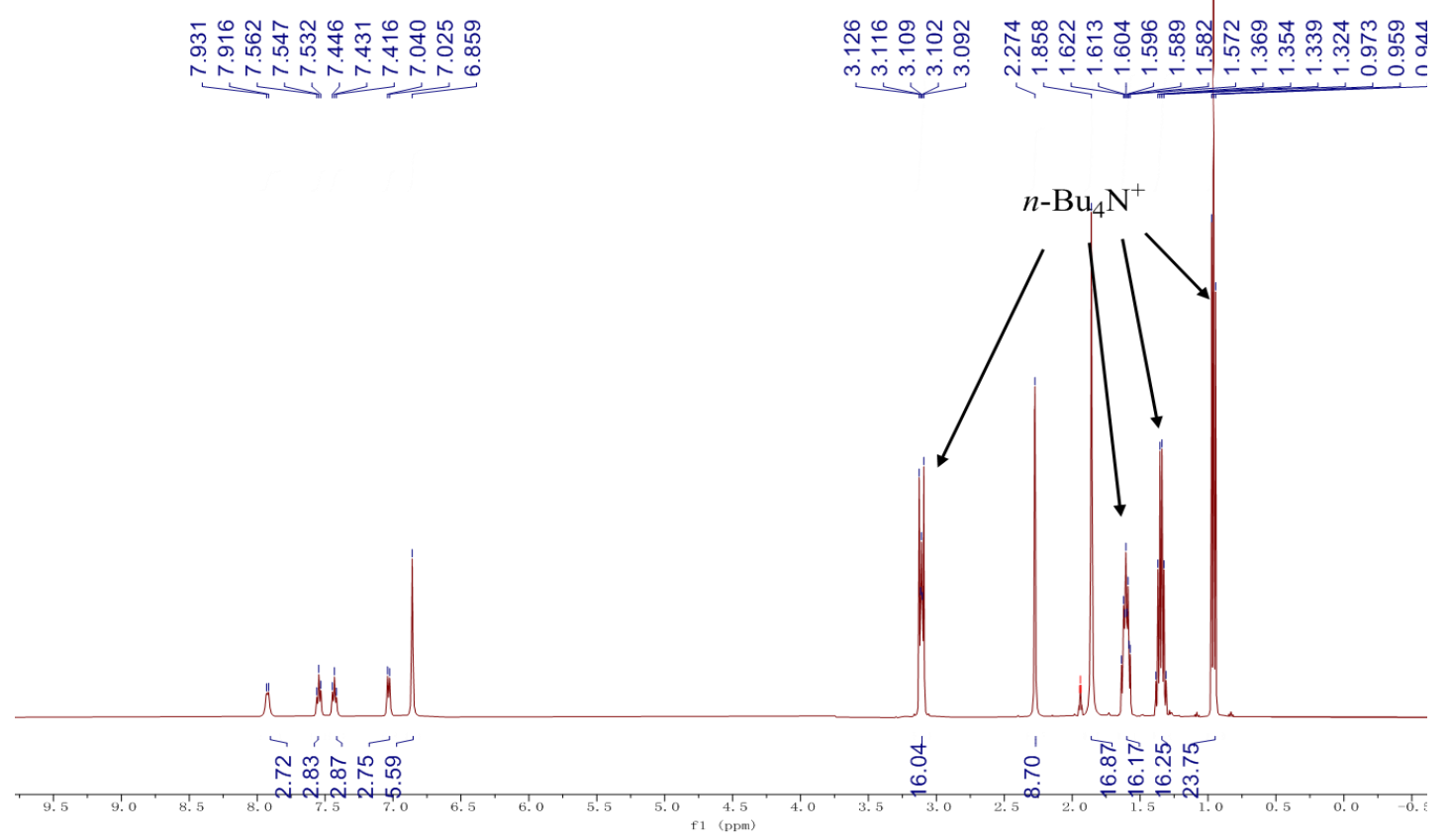

Figure S11. ${ }^{1} \mathrm{H}$ NMR spectrum of the $\left[\mathrm{Ce}(\mathrm{IV}) \mathrm{L}_{3} \mathrm{Cl}_{3}\right]^{2-}$ solution. 
${ }^{1} \mathrm{H}$ NMR $500 \mathrm{MHz}, \mathrm{CD}_{3} \mathrm{CN}$ :

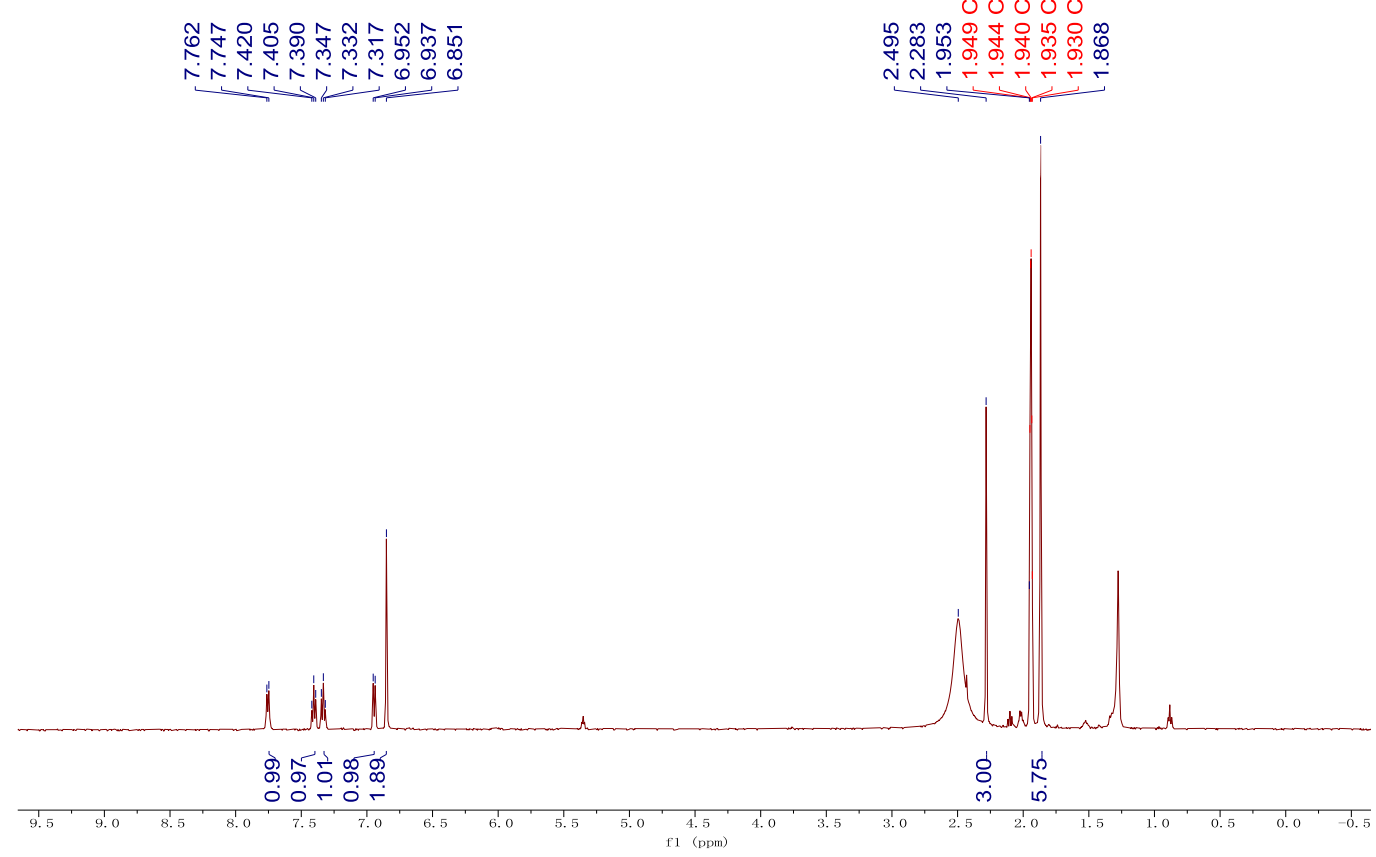

Figure S12. ${ }^{1} \mathrm{H}$ NMR spectrum of the Potassium 2',4',6'-trimethyl-[1,1'-biphenyl]-2carboxylate.

${ }^{1} \mathrm{H}$ NMR $500 \mathrm{MHz}, \mathrm{CD}_{3} \mathrm{CN}$ :

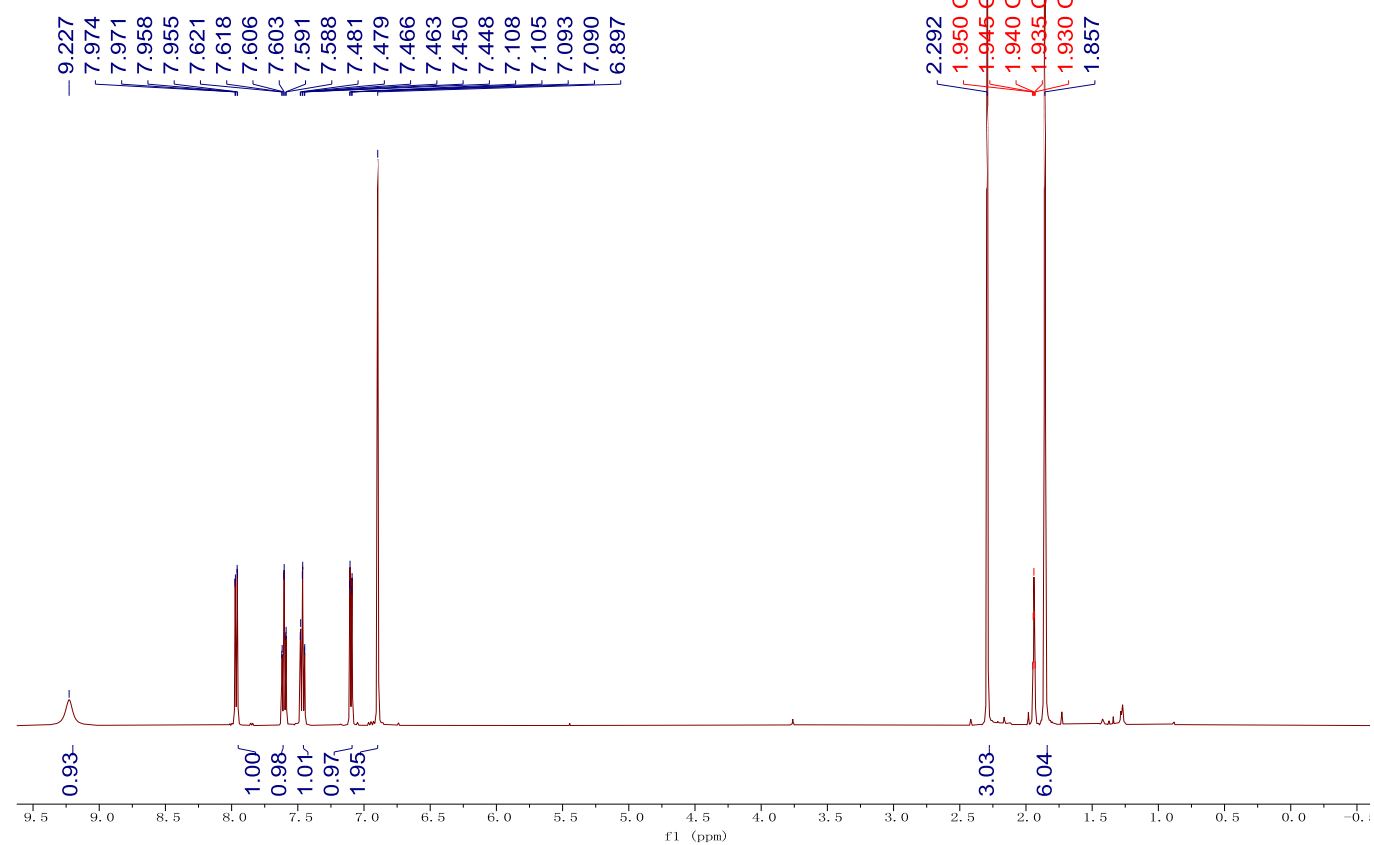

Figure S13. ${ }^{1} \mathrm{H}$ NMR spectrum of the 2',4',6'-trimethyl-[1,1'-biphenyl]-2-carboxylic acid. 


\subsection{Evaluation of the catalytic efficiency of cerium complex}

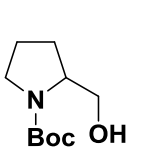

1

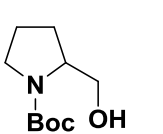

1

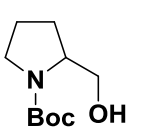

1

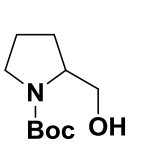

1

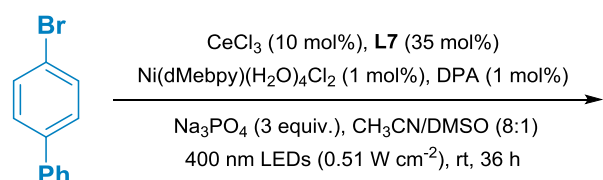

2<smiles>Pc1ccc(Br)cc1</smiles>

2

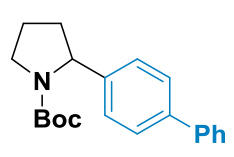

$363 \%$ yield

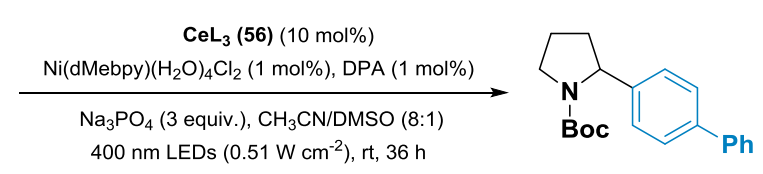

$357 \%$ yield<smiles>Brc1ccc(-c2ccccc2)cc1</smiles>

$\mathrm{CeCl}_{3}(10 \mathrm{~mol} \%), \mathbf{L} 7$ (35 mol\%)

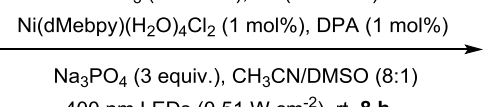

$400 \mathrm{~nm}$ LEDs $\left(0.51 \mathrm{~W} \mathrm{~cm}^{-2}\right), \mathrm{rt}, 8 \mathrm{~h}$

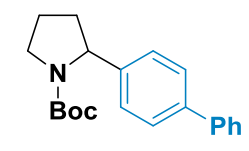

$345 \%$ yield

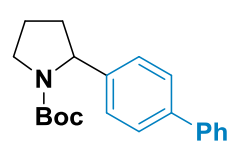

$340 \%$ yield

Figure S14. Evaluation of the catalytic efficiency of cerium complex. 


\subsection{UV-Visible Absorption Spectra}

UV-Vis spectra were collected on an Agilent Cary 5000 spectrophotometer.

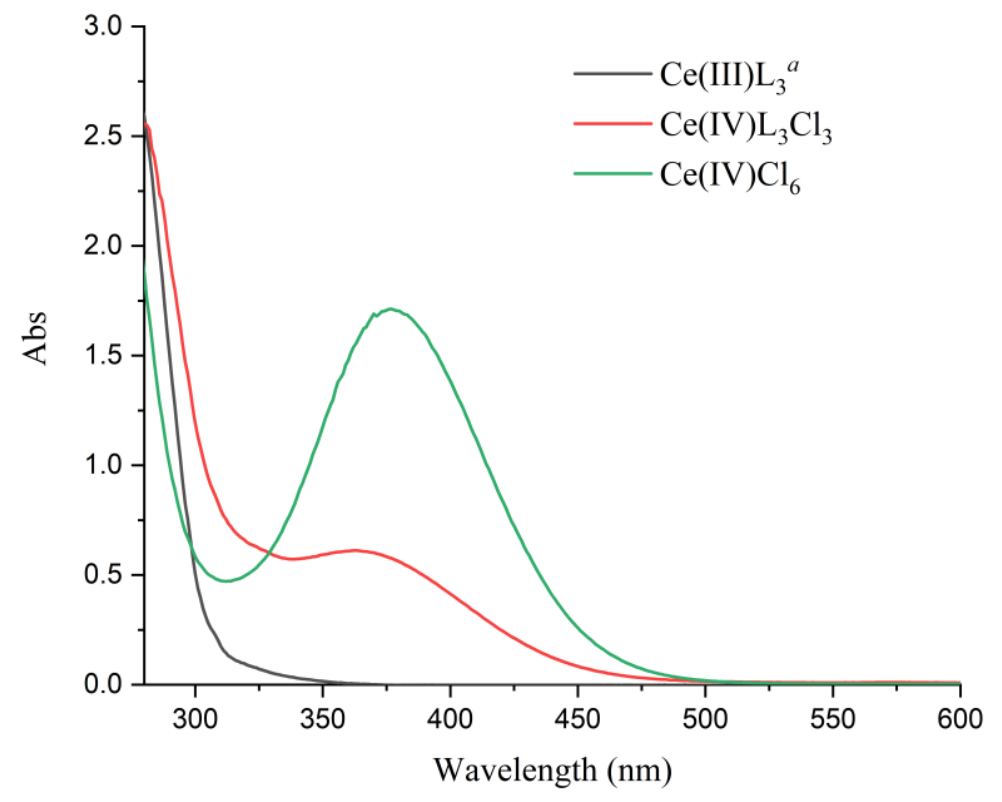

Figure S15. UV-Vis absorption of a solution of cerium(III) complex and cerium chloride complex in $\mathrm{CH}_{3} \mathrm{CN} .{ }^{a} \mathrm{CH}_{3} \mathrm{CN} / \mathrm{DMSO}$ (8:1) 


\subsection{Luminescence quenching experiments (Stern-Volmer studies)}

\subsubsection{Steady-state emission quenching experimet}

Emission intensities were recorded using a Fluorolog-3 luminescence spectrometer. Solutions of DPA were prepared and introduced to a $1 \mathrm{~cm}$ path length quartz cuvette equipped with a Teflon ${ }^{\circledR}$ septum in the glovebox (all solutions were prepared in dark). In a typical experiment, to a $0.1 \mathrm{mM}$ solution of DPA in $\mathrm{CH}_{3} \mathrm{CN} / \mathrm{DMSO}$ (8:1) was added the appropriate amount of cerium complex in a screw-top quartz cuvette in the glovebox. Then the sample was excited at $365 \mathrm{~nm}$ and the emission of the sample was collected at $428 \mathrm{~nm}$.

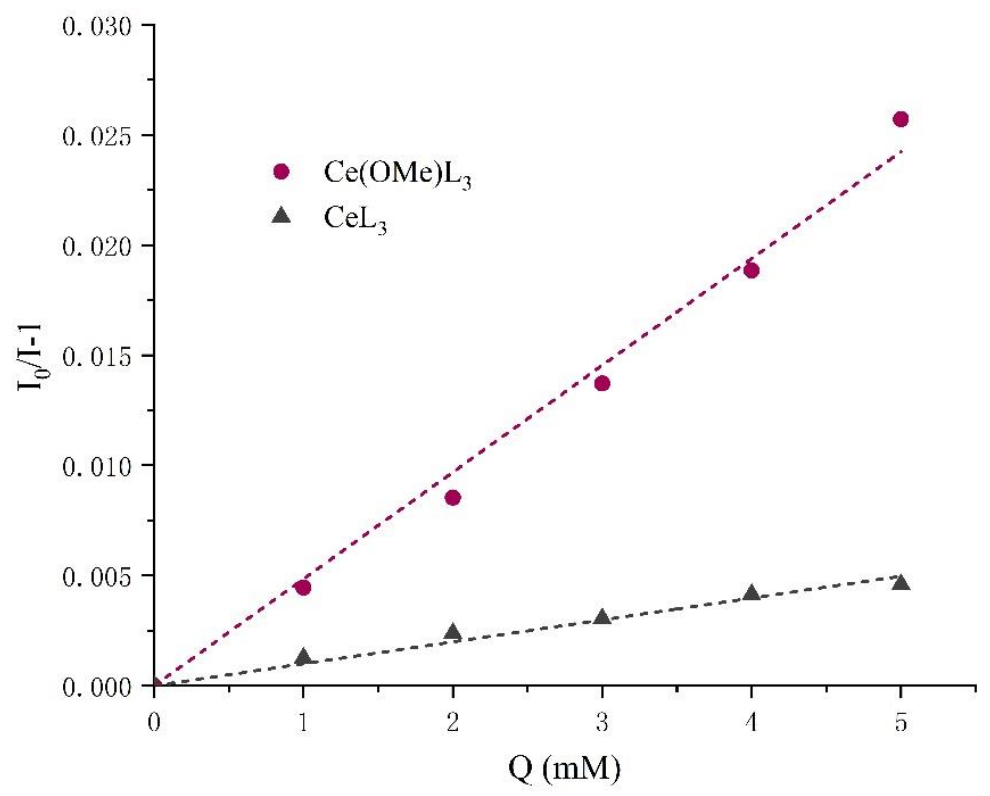

Figure S16. Stern-Volmer plot of steady-state quenching experiments.

\subsubsection{Time-resolved emission quenching experiment}

Fluorescence lifetimes were recorded using a DeltaFlex-011x time-resolved fluorescence spectrometer. Solutions of DPA were prepared and introduced to a $1 \mathrm{~cm}$ path length quartz cuvette equipped with a Teflon ${ }^{\circledR}$ septum in the glovebox (all solutions were prepared in dark). In a typical experiment, to a $0.1 \mathrm{mM}$ solution of DPA in $\mathrm{CH}_{3} \mathrm{CN} / \mathrm{DMSO}(8: 1)$ was added the appropriate amount of cerium comp in a screw- 
top quartz cuvette in the glovebox. Then the sample was excited at $367 \mathrm{~nm}$ and fluorescence lifetime of the sample was recorded at $425 \mathrm{~nm} .^{3}$

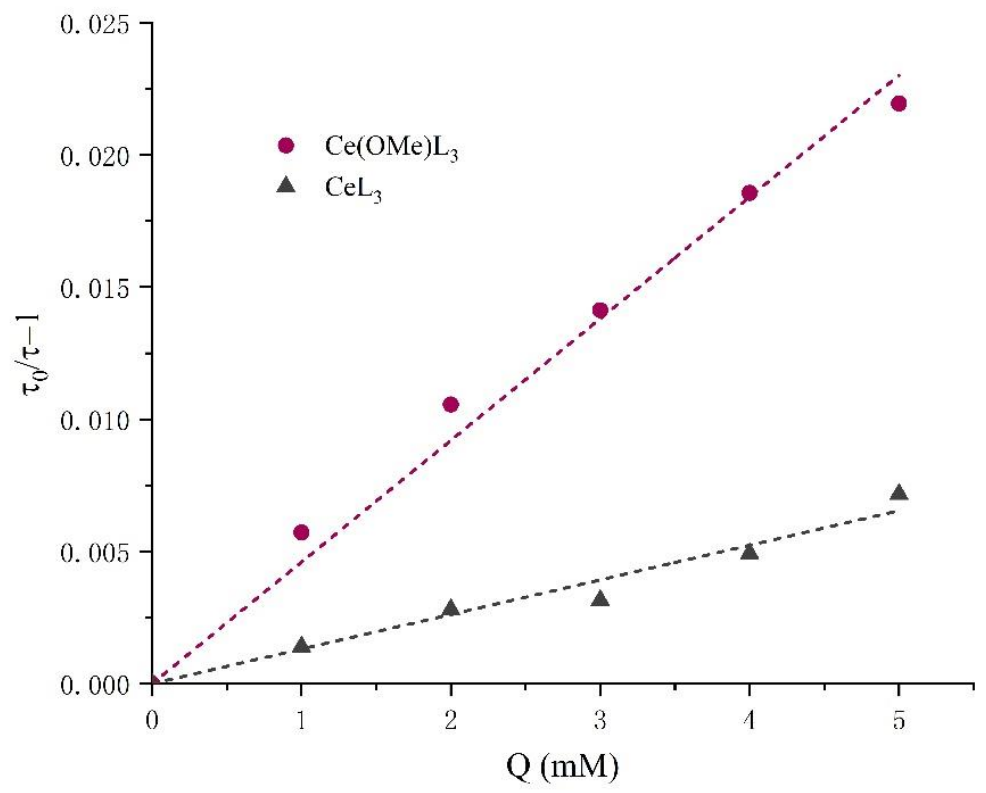

Figure S17. Stern-Volmer plot of time-resolved excited-state quenching experiments. 


\subsection{Electrochemistry}

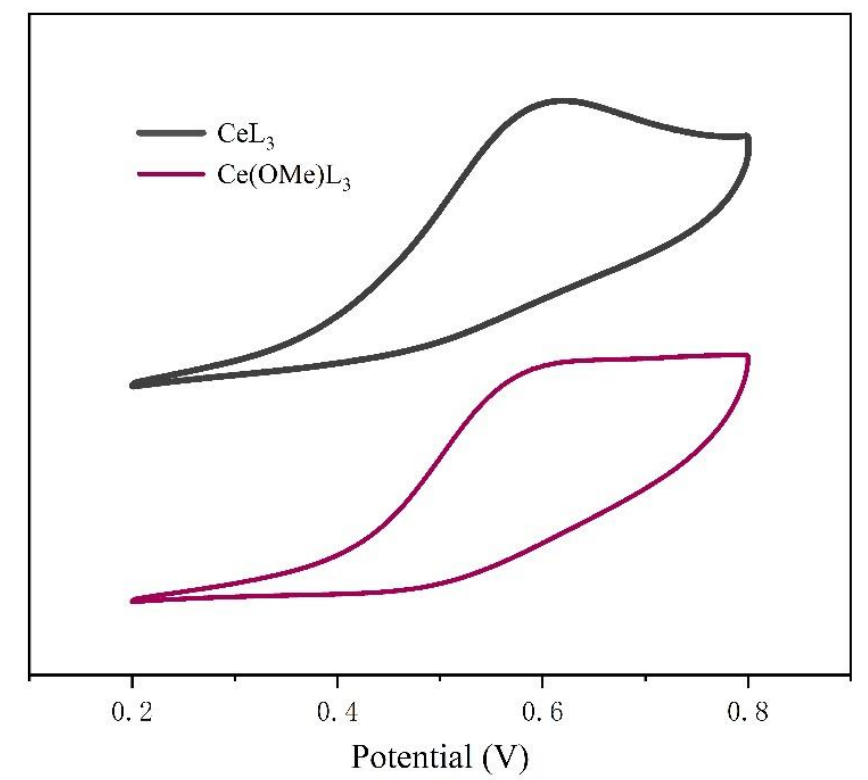

Figure S18. Cyclic voltammetry curves of cerium complexes $(c=0.05 \mathrm{M})$ in $0.1 \mathrm{M} n-\mathrm{Bu}_{4} \mathrm{NPF}_{6}$ DMSO solution. Scan Rate: 0.05 V/s. Glassy carbon electrode working electrode, $\mathrm{Ag} / \mathrm{AgCl}$ reference electrode, $\mathrm{Pt}$ wire counter electrode. 


\subsection{Reaction profile studies.}

The reaction profiles of this remote functionalization transformation with the cerium(IV) chloride complex and cerium(III) chloride are compared. To avoid introducing air into reaction mixture while taking aliquots for GC analysis, 5 identical reactions were run at the same time, and stopped over time intervals of $15 \mathrm{~min}, 30 \mathrm{~min}, 1 \mathrm{~h}, 1.5 \mathrm{~h}$, and $2 \mathrm{~h}$. The reaction mixtures were analyzed by GC against an internal standard. A marked induction period is observed for cerium(III) chloride. These observations indicate that cerium(III) chloride was functionalized as the precatalyst.

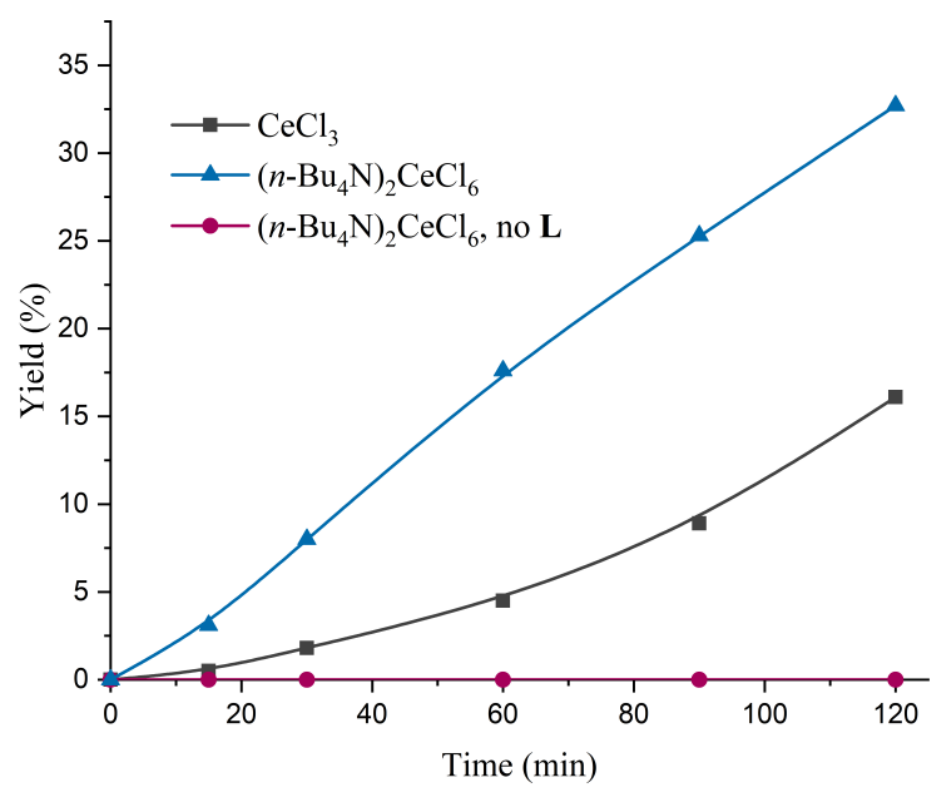

Figure S19. Reaction profile of the reactions with $\left(n-\mathrm{Bu}_{4} \mathrm{~N}\right)_{2} \mathrm{Ce}(\mathrm{IV}) \mathrm{Cl}_{6}$ and $\mathrm{CeCl}_{3}$. 


\section{Experimental procedures and spectral characterization}

\subsection{Ligand synthesis}<smiles>O=C(O)c1ccccc1I</smiles><smiles>Cc1cc(C)c(Br)c(C)c1</smiles>

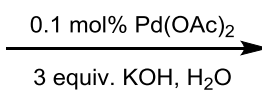<smiles>Cc1cc(O)c(-c2ccccc2C(=O)O)c([N+](=O)[O-])c1</smiles>

1.05 equiv.

2',4',6'-trimethyl-[1,1'-biphenyl]-2-carboxylic acid: A mixture of 2-iodobenzoic acid (3.72 g, $15 \mathrm{mmol}, 1$ equiv.), $75 \mathrm{ml}$ of water, mesitylboronic acid (2.58 g, $15.75 \mathrm{mmol}$, 1.05 equiv.), and $\mathrm{Pd}(\mathrm{OAc})_{2}(33.5 \mathrm{mg}, 0.15 \mathrm{mmol}, 0.01$ equiv.) was stirred at $\mathrm{rt}$ for 5 min under argon, followed by the addition of $\mathrm{KOH}(2.53 \mathrm{~g}, 45 \mathrm{mmol}, 3$ equiv.). The temperature was increased up to $75^{\circ} \mathrm{C}$. The mixture was stirred for $12 \mathrm{~h}$. After the reaction had been completed, the mixture was acidified and the product was extracted by ether. The product was isolated by flash chromatography as a white solid (1.48 g, $41 \%$ yield). ${ }^{4}$
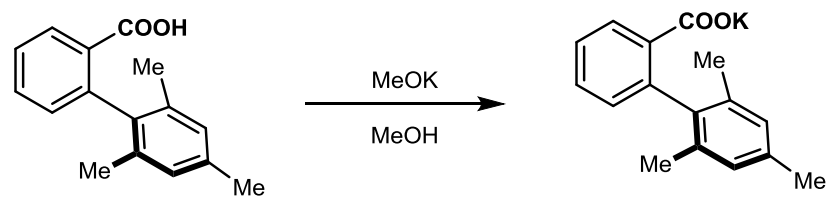

Potassium 2',4',6'-trimethyl-[1,1'-biphenyl]-2-carboxylate (L7): A solution of $\mathrm{CH}_{3} \mathrm{OK}(350 \mathrm{mg}, 5 \mathrm{mmol})$ in $\mathrm{CH}_{3} \mathrm{OH}(10 \mathrm{~mL})$ was added to a solution of 2',4',6'trimethyl-[1,1'-biphenyl]-2-carboxylic acid (1.2 g, $5 \mathrm{mmol})$ in $\mathrm{CH}_{3} \mathrm{OH}(20 \mathrm{~mL})$. The solution was stirred for $4 \mathrm{~h}$. Then the solution was evaporated and diethyl ether $(50 \mathrm{~mL})$ was added to the remaining white solid and the mixture was stirred vigorously for $2 \mathrm{~h}$. Then the white solid was filtered and washed twice with diethyl ether and dried under vacuum giving $1.33 \mathrm{~g}(95 \%, 4.78 \mathrm{mmol})$ pure product as a white powder. 


\subsection{Experimental procedures and spectral characterization of the products}

General procedure: In a glovebox, a 8-mL vial equipped with a magnetic stirring bar was charged with tert-butyl 2-(hydroxymethyl)pyrrolidine-1-carboxylate ( $80.5 \mathrm{mg}, 0.4$ mmol, 2.0 equiv.), 4-bromo-1,1'-biphenyl (46.6 mg, $0.2 \mathrm{mmol}, 1.0$ equiv.), $\mathrm{Ni}(\mathrm{dMebpy})\left(\mathrm{H}_{2} \mathrm{O}\right)_{4} \mathrm{Cl}_{2}(0.8 \mathrm{mg}, 0.002 \mathrm{mmol}, 0.01$ equiv.), DPA (0.7 mg, $0.002 \mathrm{mmol}$, 0.01 equiv.), $\mathbf{L} 4$ (20.0 mg, 0.07 mmol, 0.35 equiv.), $\mathrm{CeCl}_{3}$ (4.9 mg, $0.02 \mathrm{mmol}, 0.1$ equiv.), $\mathrm{Na}_{3} \mathrm{PO}_{4}$ (98.4 mg, $0.6 \mathrm{mmol}, 3$ equiv.), $\mathrm{CH}_{3} \mathrm{CN}$ (2 mL) and DMSO (0.25 mL). The vial was sealed with a polytetrafluoroethylene-lined cap, then irradiated with 400 $\mathrm{nm} \operatorname{LED}\left(0.51 \mathrm{~W} / \mathrm{cm}^{2}\right)$. The reaction was stirred under irradiation at ambient temperature for the $36 \mathrm{~h}$. The reaction mixture was concentrated in vacuo. Purification by flash chromatography on silica gel provided the desired product.

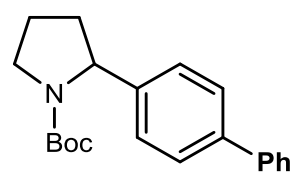

3

tert-butyl 2-([1,1'-biphenyl]-4-yl)pyrrolidine-1-carboxylate (3): According to the general procedure, tert-butyl 2-(hydroxymethyl)pyrrolidine-1-carboxylate $(80.5 \mathrm{mg}$, 0.4 mmol, 2.0 equiv.), 4-bromo-1,1'-biphenyl (46.6 mg, $0.2 \mathrm{mmol}, 1.0$ equiv.), $\mathrm{Ni}(\mathrm{dMebpy})\left(\mathrm{H}_{2} \mathrm{O}\right)_{4} \mathrm{Cl}_{2}(0.8 \mathrm{mg}, 0.002 \mathrm{mmol}, 0.01$ equiv.), DPA (0.7 mg, $0.002 \mathrm{mmol}$, 0.01 equiv.), $\mathbf{L 4}$ (20.0 mg, $0.07 \mathrm{mmol}, 0.35$ equiv.), $\mathrm{CeCl}_{3}$ (4.9 mg, $0.02 \mathrm{mmol}, 0.1$ equiv.), $\mathrm{Na}_{3} \mathrm{PO}_{4}$ (98.4 mg, $0.6 \mathrm{mmol}, 3$ equiv.), $\mathrm{CH}_{3} \mathrm{CN}(2 \mathrm{~mL})$ and DMSO (0.25 mL) were used. After $36 \mathrm{~h}$, the product was isolated by flash chromatography as a colorless oil (60 mg, 93\%). ${ }^{1} \mathbf{H}$ NMR (500 MHz, Chloroform-d) $\delta 7.59(\mathrm{t}, J=10.2 \mathrm{~Hz}, 2 \mathrm{H})$, $7.53(\mathrm{~d}, J=7.8 \mathrm{~Hz}, 2 \mathrm{H}), 7.43(\mathrm{t}, J=7.8 \mathrm{~Hz}, 2 \mathrm{H}), 7.33(\mathrm{t}, J=7.3 \mathrm{~Hz}, 1 \mathrm{H}), 7.27-7.21$ $(\mathrm{m}, 2 \mathrm{H}), 4.80(\mathrm{~m}, 1 \mathrm{H}), 3.65(\mathrm{~m}, 2 \mathrm{H}), 2.34(\mathrm{~m}, 1 \mathrm{H}), 1.89(\mathrm{~m}, 3 \mathrm{H}), 1.47(\mathrm{~s}, 3 \mathrm{H}), 1.20(\mathrm{~s}$, 6H). ${ }^{13}$ C NMR (125 MHz, Chloroform-d) $\delta$ 154.6, 144.2, 141.3, 140.8, 139.3, 128.7, 127.2, 127.1\&127.0, $126.9 \& 126.8, \quad 126.0 \& 125.7, \quad 79.3, \quad 61.1 \& 60.4, \quad 47.3 \& 47.1$, 36.0\&34.8, 28.5\&28.1, 23.5\&23.2. HRMS (ESI+): calcd for $\mathrm{C}_{21} \mathrm{H}_{25} \mathrm{NO}_{2} \mathrm{Na}(\mathrm{M}+\mathrm{Na})$ 346.1778 , found 346.1781 . 


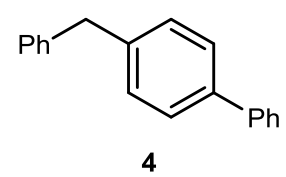

4-benzyl-1,1'-biphenyl (4): According to the general procedure, 2-phenylethan-1-ol (48.9 mg, $0.4 \mathrm{mmol}, 2.0$ equiv.), 4-bromo-1,1'-biphenyl (46.6 mg, $0.2 \mathrm{mmol}, 1.0$ equiv.), $\mathrm{Ni}(\mathrm{dMebpy})\left(\mathrm{H}_{2} \mathrm{O}\right){ }_{4} \mathrm{Cl}_{2}(0.8 \mathrm{mg}, 0.002 \mathrm{mmol}, 0.01$ equiv. $)$, DPA (0.7 mg, $0.002 \mathrm{mmol}$, 0.01 equiv.), $\mathbf{L} 4$ (20.0 mg, 0.07 mmol, 0.35 equiv.), $\mathrm{CeCl}_{3}$ (4.9 mg, $0.02 \mathrm{mmol}, 0.1$ equiv.), $\mathrm{Na}_{3} \mathrm{PO}_{4}$ (98.4 mg, $0.6 \mathrm{mmol}, 3$ equiv.), $\mathrm{CH}_{3} \mathrm{CN}(2 \mathrm{~mL})$ and DMSO (0.25 mL) were used. After $36 \mathrm{~h}$, the product was isolated by flash chromatography as a white solid (42 mg, 86\%). ${ }^{1}$ H NMR (500 MHz, Chloroform-d) $\delta 7.56(\mathrm{~d}, J=7.6 \mathrm{~Hz}, 2 \mathrm{H})$, $7.51(\mathrm{~d}, J=7.7 \mathrm{~Hz}, 2 \mathrm{H}), 7.41(\mathrm{t}, J=7.5 \mathrm{~Hz}, 2 \mathrm{H}), 7.30$ (q, $J=7.3 \mathrm{~Hz}, 3 \mathrm{H}), 7.24(\mathrm{dd}, J$ $=15.0,7.7 \mathrm{~Hz}, 5 \mathrm{H}), 4.01(\mathrm{~s}, 2 \mathrm{H}) .{ }^{13} \mathrm{C}$ NMR (125 MHz, Chloroform-d) $\delta$ 141.0, 140.2, 139.0, 129.3, 128.9, 128.7, 128.5, 127.2, 127.1, 127.0, 126.1, 41.6. HRMS (EI-QTOF): calcd for $\mathrm{C}_{19} \mathrm{H}_{16} 244.1252$, found 244.1248.

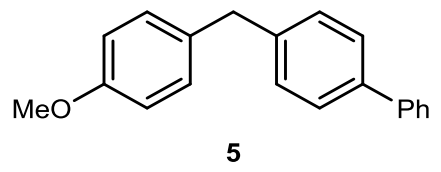

4-(4-methoxybenzyl)-1,1'-biphenyl (5): According to the general procedure, 2-(4methoxyphenyl)ethan-1-ol (60.9 mg, 0.4 mmol, 2.0 equiv.), 4-bromo-1,1'-biphenyl (46.6 mg, 0.2 mmol, 1.0 equiv.), $\mathrm{Ni}(\mathrm{dMebpy})\left(\mathrm{H}_{2} \mathrm{O}\right)_{4} \mathrm{Cl}_{2}(0.8 \mathrm{mg}, 0.002 \mathrm{mmol}, 0.01$ equiv.), DPA (0.7 mg, $0.002 \mathrm{mmol}, 0.01$ equiv.), L4 (20.0 mg, $0.07 \mathrm{mmol}, 0.35$ equiv.), $\mathrm{CeCl}_{3}$ (4.9 mg, 0.02 mmol, 0.1 equiv.), $\mathrm{Na}_{3} \mathrm{PO}_{4}$ (98.4 mg, 0.6 mmol, 3 equiv.), $\mathrm{CH}_{3} \mathrm{CN}$ $(2 \mathrm{~mL})$ and DMSO $(0.25 \mathrm{~mL})$ were used. After $36 \mathrm{~h}$, the product was isolated by flash chromatography as a white solid (44 mg, 80\%). ${ }^{1} \mathbf{H}$ NMR (500 MHz, Chloroform-d) $\delta 7.57-7.56(\mathrm{~m}, 2 \mathrm{H}), 7.52-7.50(\mathrm{~m}, 2 \mathrm{H}), 7.43-7.40(\mathrm{~m}, 2 \mathrm{H}), 7.34-7.30(\mathrm{~m}, 1 \mathrm{H})$, $7.25-7.24(\mathrm{~m}, 2 \mathrm{H}), 7.15-7.14(\mathrm{~m}, 2 \mathrm{H}), 6.86-6.84(\mathrm{~m}, 2 \mathrm{H}), 3.96(\mathrm{~s}, 2 \mathrm{H}), 3.78(\mathrm{~s}$, 3H). ${ }^{13}$ C NMR (125 MHz, Chloroform-d) $\delta$ 158.0, 141.0, 140.7, 138.9, 133.1, 129.9, 
129.2, 128.7, 127.2, 127.1, 127.0, 113.9, 55.2, 40.7. HRMS (EI-QTOF): calcd for $\mathrm{C}_{20} \mathrm{H}_{18} \mathrm{O} 274.1358$, found 274.1355.

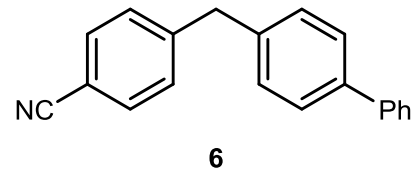

4-([1,1'-biphenyl]-4-ylmethyl)benzonitrile (6): According to the general procedure, 4-(2-hydroxyethyl)benzonitrile (58.9 mg, $0.4 \mathrm{mmol}, 2.0$ equiv.), 4-bromo-1,1'-biphenyl (46.6 mg, 0.2 mmol, 1.0 equiv.), $\mathrm{Ni}(\mathrm{dMebpy})\left(\mathrm{H}_{2} \mathrm{O}\right)_{4} \mathrm{Cl}_{2}(0.8 \mathrm{mg}, 0.002 \mathrm{mmol}, 0.01$ equiv.), DPA (0.7 mg, $0.002 \mathrm{mmol}, 0.01$ equiv.), L4 (20.0 mg, 0.07 mmol, 0.35 equiv.), $\mathrm{CeCl}_{3}$ (4.9 mg, 0.02 mmol, 0.1 equiv.), $\mathrm{Na}_{3} \mathrm{PO}_{4}$ (98.4 mg, 0.6 mmol, 3 equiv.), $\mathrm{CH}_{3} \mathrm{CN}$ $(2 \mathrm{~mL})$ and DMSO $(0.25 \mathrm{~mL})$ were used. After $36 \mathrm{~h}$, the product was isolated by flash chromatography as a white solid (38 mg, 70\%). ${ }^{1}$ H NMR (500 MHz, Chloroform-d) $\delta 7.60-7.53(\mathrm{~m}, 5 \mathrm{H}), 7.45-7.42(\mathrm{~m}, 2 \mathrm{H}), 7.36-7.32(\mathrm{~m}, 3 \mathrm{H}), 7.2-7.22(\mathrm{~m}, 3 \mathrm{H})$, 4.08 (s, 2H). ${ }^{13}$ C NMR (125 MHz, Chloroform-d) $\delta$ 146.6, 140.7, 139.7, 138.4, 132.4, 129.7, 129.4, 128.8, 127.5, 127.3, 127.0, 119.0, 110.1, 41.6. HRMS (EI-QTOF): calcd for $\mathrm{C}_{20} \mathrm{H}_{15} \mathrm{~N} 269.1204$, found 269.1202.

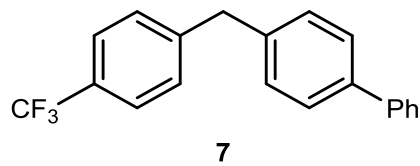

4-(4-(trifluoromethyl)benzyl)-1,1'-biphenyl (7): According to the general procedure, 2-(4-(trifluoromethyl)phenyl)ethan-1-ol (76.1 mg, 0.4 mmol, 2.0 equiv.), 4-bromo-1,1'biphenyl (46.6 mg, $0.2 \mathrm{mmol}, 1.0$ equiv.), $\mathrm{Ni}(\mathrm{dMebpy})\left(\mathrm{H}_{2} \mathrm{O}\right)_{4} \mathrm{Cl}_{2}(0.8 \mathrm{mg}, 0.002 \mathrm{mmol}$, 0.01 equiv.), DPA (0.7 mg, $0.002 \mathrm{mmol}, 0.01$ equiv.), $\mathbf{L 4}$ (20.0 mg, $0.07 \mathrm{mmol}, 0.35$ equiv.), $\mathrm{CeCl}_{3}$ (4.9 mg, $0.02 \mathrm{mmol}, 0.1$ equiv.), $\mathrm{Na}_{3} \mathrm{PO}_{4}$ (98.4 mg, $0.6 \mathrm{mmol}, 3$ equiv.), $\mathrm{CH}_{3} \mathrm{CN}(2 \mathrm{~mL})$ and DMSO $(0.25 \mathrm{~mL})$ were used. After $36 \mathrm{~h}$, the product was isolated by flash chromatography as a white solid (44 mg, 71\%). ${ }^{1} \mathbf{H}$ NMR (500 MHz, Chloroform- $d$ ) $\delta 7.58-7.52(\mathrm{~m}, 6 \mathrm{H}), 7.42(\mathrm{t}, J=7.5 \mathrm{~Hz}, 2 \mathrm{H}), 7.33(\mathrm{dd}, J=7.7,4.9$ 
Hz, 3H), 7.25 (s, 2H), 4.06 (s, 2H). ${ }^{13}$ C NMR (125 MHz, Chloroform-d) $\delta$ 145.1, 140.8, 139.4, 139.0, 129.3, 129.2, 128.8, 128.5 (q, $J=31.5 \mathrm{~Hz}), 127.4,127.2$, 127.0, 126.8, 125.5 (q, $J=3.8 \mathrm{~Hz}), 124.3$ (q, $J=272 \mathrm{~Hz}), 41.3 .{ }^{19} \mathbf{F}$ NMR (470 MHz, Chloroform- $d$ ) $\delta$-62.29. HRMS (EI-QTOF): calcd for $\mathrm{C}_{20} \mathrm{H}_{15} \mathrm{~F}_{3} 312.1126$, found 312.1127 .

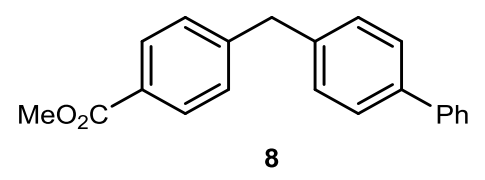

methyl 4-([1,1'-biphenyl]-4-ylmethyl)benzoate (8): According to the general procedure, methyl 4-(2-hydroxyethyl)benzoate $(72.1 \mathrm{mg}, 0.4 \mathrm{mmol}, 2.0$ equiv.), 4bromo-1,1'-biphenyl (46.6 mg, $0.2 \mathrm{mmol}, 1.0$ equiv.), $\mathrm{Ni}(\mathrm{dMebpy})\left(\mathrm{H}_{2} \mathrm{O}\right)_{4} \mathrm{Cl}_{2}(0.8 \mathrm{mg}$, $0.002 \mathrm{mmol}, 0.01$ equiv.), DPA (0.7 mg, $0.002 \mathrm{mmol}, 0.01$ equiv.), L4 (20.0 mg, 0.07 mmol, 0.35 equiv.), $\mathrm{CeCl}_{3}$ (4.9 mg, 0.02 mmol, 0.1 equiv.), $\mathrm{Na}_{3} \mathrm{PO}_{4}$ (98.4 mg, 0.6 mmol, 3 equiv.), $\mathrm{CH}_{3} \mathrm{CN}(2 \mathrm{~mL})$ and DMSO $(0.25 \mathrm{~mL})$ were used. After $36 \mathrm{~h}$, the product was isolated by flash chromatography as a white solid (40 mg, 66\%). ${ }^{1}$ H NMR (500 MHz, Chloroform-d $) \delta 7.92-7.90(\mathrm{~m}, 2 \mathrm{H}), 7.50(\mathrm{~d}, J=7.6 \mathrm{~Hz}, 2 \mathrm{H}), 7.48-7.42(\mathrm{~m}, 2 \mathrm{H})$, $7.35(\mathrm{t}, J=7.5 \mathrm{~Hz}, 2 \mathrm{H}), 7.26(\mathrm{~d}, J=7.5 \mathrm{~Hz}, 1 \mathrm{H}), 7.22(\mathrm{~d}, J=7.9 \mathrm{~Hz}, 2 \mathrm{H}), 7.17(\mathrm{~d}, J=$ $8.2 \mathrm{~Hz}, 2 \mathrm{H}), 4.00$ (s, 2H), 3.83 (s, 3H). ${ }^{13}$ C NMR (125 MHz, Chloroform-d) $\delta$ 167.0, $146.4,140.8,139.3,139.2,129.9,129.3,129.0,128.7,128.1,127.3,127.2$, 127.0, 52.0, 41.5. HRMS (EI-QTOF): calcd for $\mathrm{C}_{21} \mathrm{H}_{18} \mathrm{O}_{2} 302.1307$, found 302.1308 .<smiles>c1ccc(-c2ccc(Cc3ccc4ccccc4c3)cc2)cc1</smiles>

9

2-([1,1'-biphenyl]-4-ylmethyl)naphthalene (9): According to the general procedure, 2-(naphthalen-2-yl)ethan-1-ol (68.9 mg, 0.4 mmol, 2.0 equiv.), 4-bromo-1,1'-biphenyl (46.6 mg, $0.2 \mathrm{mmol}, 1.0$ equiv.), $\mathrm{Ni}(\mathrm{dMebpy})\left(\mathrm{H}_{2} \mathrm{O}\right)_{4} \mathrm{Cl}_{2}(0.8 \mathrm{mg}, 0.002 \mathrm{mmol}, 0.01$ equiv.), DPA (0.7 mg, $0.002 \mathrm{mmol}, 0.01$ equiv.), L4 (20.0 mg, $0.07 \mathrm{mmol}, 0.35$ equiv.), 
$\mathrm{CeCl}_{3}$ (4.9 mg, $0.02 \mathrm{mmol}, 0.1$ equiv.), $\mathrm{Na}_{3} \mathrm{PO}_{4}$ (98.4 mg, 0.6 mmol, 3 equiv.), $\mathrm{CH}_{3} \mathrm{CN}$ $(2 \mathrm{~mL})$ and DMSO $(0.25 \mathrm{~mL})$ were used. After $36 \mathrm{~h}$, the product was isolated by flash chromatography as a white solid (49 mg, 83\%). ${ }^{1}$ H NMR (500 MHz, Chloroform-d) $\delta 7.83-7.74(\mathrm{~m}, 3 \mathrm{H}), 7.67(\mathrm{~s}, 1 \mathrm{H}), 7.57$ (d, J = 7.6 Hz, 2H), $7.52(\mathrm{~d}, J=7.8 \mathrm{~Hz}, 2 \mathrm{H})$, $7.42(\mathrm{dt}, J=12.1,7.5 \mathrm{~Hz}, 4 \mathrm{H}), 7.37-7.26(\mathrm{~m}, 4 \mathrm{H}), 4.18(\mathrm{~s}, 2 \mathrm{H}) .{ }^{13} \mathbf{C}$ NMR (125 MHz, Chloroform- $d) \delta 140.9,140.1,139.0,138.5,133.6,132.1,129.4,128.7,128.1,127.6$, 127.5, 127.2, 127.1, 127.0, 126.0, 125.4, 41.7. HRMS (EI-QTOF): calcd for $\mathrm{C}_{23} \mathrm{H}_{18}$ 294.1409, found 294.1405.

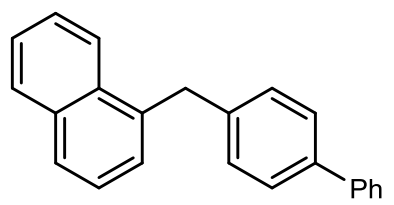

10

1-(2-([1,1'-biphenyl]-4-yl)ethyl)naphthalene (10): According to the general procedure, 2-(naphthalen-1-yl)ethan-1-ol (68.9 mg, 0.4 mmol, 2.0 equiv.), 4-bromo1,1'-biphenyl (46.6 mg, $0.2 \mathrm{mmol}, 1.0$ equiv.), $\mathrm{Ni}(\mathrm{dMebpy})\left(\mathrm{H}_{2} \mathrm{O}\right)_{4} \mathrm{Cl}_{2}(0.8 \mathrm{mg}, 0.002$ mmol, 0.01 equiv.), DPA (0.7 mg, $0.002 \mathrm{mmol}, 0.01$ equiv.), L4 (20.0 mg, $0.07 \mathrm{mmol}$, 0.35 equiv.), $\mathrm{CeCl}_{3}$ (4.9 mg, $0.02 \mathrm{mmol}, 0.1$ equiv.), $\mathrm{Na}_{3} \mathrm{PO}_{4}$ (98.4 mg, $0.6 \mathrm{mmol}, 3$ equiv.), $\mathrm{CH}_{3} \mathrm{CN}(2 \mathrm{~mL})$ and DMSO $(0.25 \mathrm{~mL})$ were used. After $36 \mathrm{~h}$, the product was isolated by flash chromatography as a white solid (37 mg, 63\%). ${ }^{1} \mathbf{H}$ NMR (500 MHz, Chloroform-d) $\delta 8.08-7.99(\mathrm{~m}, 1 \mathrm{H}), 7.92-7.85(\mathrm{~m}, 1 \mathrm{H}), 7.78(\mathrm{~d}, J=8.4 \mathrm{~Hz}, 1 \mathrm{H})$, $7.56(\mathrm{~d}, J=7.4 \mathrm{~Hz}, 2 \mathrm{H}), 7.45(\mathrm{~m}, 7 \mathrm{H}), 7.37-7.29(\mathrm{~m}, 2 \mathrm{H}), 7.27(\mathrm{~d}, J=8.4 \mathrm{~Hz}, 2 \mathrm{H})$, 4.50 (s, 2H). ${ }^{13}$ C NMR (125 MHz, Chloroform- $\left.d\right) \delta$ 140.9, 139.7, 139.0, 136.5, 133.9, $132.1,129.1,128.7,127.4,127.2,127.1,127.0,126.0,125.9,125.6,124.3,38.7$. HRMS (EI-QTOF): calcd for $\mathrm{C}_{23} \mathrm{H}_{18} 294.1409$, found 294.1410.

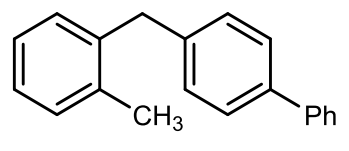

11 
4-(2-methylbenzyl)-1,1'-biphenyl (11): According to the general procedure, 2-(otolyl)ethan-1-ol (54.5 mg, $0.4 \mathrm{mmol}, 2.0$ equiv.), 4-bromo-1,1'-biphenyl (46.6 mg, 0.2 mmol, 1.0 equiv.), $\mathrm{Ni}(\mathrm{dMebpy})\left(\mathrm{H}_{2} \mathrm{O}\right)_{4} \mathrm{Cl}_{2}(0.8 \mathrm{mg}, 0.002 \mathrm{mmol}, 0.01$ equiv. $), \mathrm{DPA}(0.7$ mg, 0.002 mmol, 0.01 equiv.), $\mathbf{L 4}$ (20.0 mg, 0.07 mmol, 0.35 equiv.), $\mathrm{CeCl}_{3}$ (4.9 mg, 0.02 mmol, 0.1 equiv.), $\mathrm{Na}_{3} \mathrm{PO}_{4}$ (98.4 mg, $0.6 \mathrm{mmol}, 3$ equiv.), $\mathrm{CH}_{3} \mathrm{CN}$ (2 mL) and DMSO $(0.25 \mathrm{~mL})$ were used. After $36 \mathrm{~h}$, the product was isolated by flash chromatography as a white solid (39 mg, 76\%). ${ }^{1}$ H NMR (500 MHz, Chloroform- $d$ ) $\delta 7.52-7.47(\mathrm{~m}, 2 \mathrm{H}), 7.45-7.40(\mathrm{~m}, 2 \mathrm{H}), 7.35(\mathrm{~m}, 2 \mathrm{H}), 7.27-7.22(\mathrm{~m}, 1 \mathrm{H}), 7.15-$ 7.04 (m, 6H), 3.95 (s, 2H), 2.20 (s, 3H). 13C NMR (125 MHz, Chloroform-d) $\delta$ 141.0, 139.5, 138.8, 136.7, 130.3, 129.9, 129.1, 128.7, 127.1, 127.0, 126.5, 126.0, 39.1, 19.7 .

HRMS (EI-QTOF): calcd for $\mathrm{C}_{20} \mathrm{H}_{18} 258.1409$, found 258.1417 .

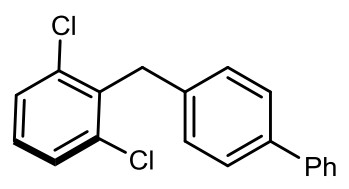

12

4-(2,6-dichlorobenzyl)-1,1'-biphenyl (12): According to the general procedure, 2(2,6-dichlorophenyl)ethan-1-ol (76.4 mg, 0.4 mmol, 2.0 equiv.), 4-bromo-1,1'-biphenyl (46.6 mg, $0.2 \mathrm{mmol}, 1.0$ equiv.), $\mathrm{Ni}(\mathrm{dMebpy})\left(\mathrm{H}_{2} \mathrm{O}\right)_{4} \mathrm{Cl}_{2}(3.7 \mathrm{mg}, 0.01 \mathrm{mmol}, 0.05$ equiv.), DPA (0.7 mg, $0.002 \mathrm{mmol}, 0.01$ equiv.), L4 (20.0 mg, $0.07 \mathrm{mmol}, 0.35$ equiv.), $\mathrm{CeCl}_{3}$ (4.9 mg, 0.02 mmol, 0.1 equiv.), $\mathrm{Na}_{3} \mathrm{PO}_{4}$ (98.4 mg, $0.6 \mathrm{mmol}, 3$ equiv.), $\mathrm{CH}_{3} \mathrm{CN}$ $(2 \mathrm{~mL})$ and DMSO $(0.25 \mathrm{~mL})$ were used. After $36 \mathrm{~h}$, the product was isolated by flash chromatography as a white solid (38 mg, 61\%). ${ }^{\mathbf{1}} \mathbf{H}$ NMR (500 MHz, Chloroform-d) $\delta 7.56(\mathrm{~d}, J=7.7 \mathrm{~Hz}, 2 \mathrm{H}), 7.50(\mathrm{~d}, J=7.8 \mathrm{~Hz}, 2 \mathrm{H}), 7.41(\mathrm{t}, J=7.5 \mathrm{~Hz}, 2 \mathrm{H}), 7.34(\mathrm{dd}$, $J=14.6,7.7 \mathrm{~Hz}, 3 \mathrm{H}), 7.28(\mathrm{~s}, 2 \mathrm{H}), 7.15(\mathrm{t}, J=8.0 \mathrm{~Hz}, 1 \mathrm{H}), 4.38(\mathrm{~s}, 2 \mathrm{H}) .{ }^{13} \mathbf{C}$ NMR (125 MHz, Chloroform-d) $\delta$ 140.9, 139.2, 137.2, 136.6, 136.1, 128.7, 128.4, 128.2, 127.1, 127.0, 36.3. HRMS (EI-QTOF): calcd for $\mathrm{C}_{19} \mathrm{H}_{14} \mathrm{Cl}_{2} 312.0473$, found 312.0469. 


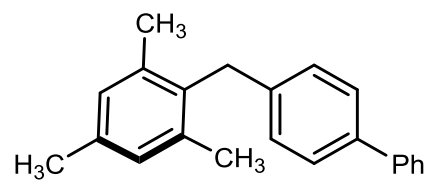

13

4-(2,4,6-trimethylbenzyl)-1,1'-biphenyl (13): According to the general procedure, 2mesitylethan-1-ol (65.7 mg, 0.4 mmol, 2.0 equiv.), 4-bromo-1,1'-biphenyl (46.6 mg, $0.2 \mathrm{mmol}, 1.0$ equiv.), $\mathrm{Ni}(\mathrm{dMebpy})\left(\mathrm{H}_{2} \mathrm{O}\right)_{4} \mathrm{Cl}_{2}(3.7 \mathrm{mg}, 0.01 \mathrm{mmol}, 0.05$ equiv.), DPA (0.7 mg, 0.002 mmol, 0.01 equiv.), $\mathbf{L 4}$ (20.0 mg, 0.07 mmol, 0.35 equiv.), $\mathrm{CeCl}_{3}$ (4.9 mg, 0.02 mmol, 0.1 equiv.), $\mathrm{Na}_{3} \mathrm{PO}_{4}$ (98.4 mg, $0.6 \mathrm{mmol}, 3$ equiv.), $\mathrm{CH}_{3} \mathrm{CN}$ (2 mL) and DMSO $(0.25 \mathrm{~mL})$ were used. After $36 \mathrm{~h}$, the product was isolated by flash chromatography as a white solid (29 mg, 51\%). ${ }^{1} \mathbf{H}$ NMR (500 MHz, Chloroform-d) $\delta 7.57-7.52(\mathrm{~m}, 2 \mathrm{H}), 7.48-7.44(\mathrm{~m}, 2 \mathrm{H}), 7.41(\mathrm{t}, J=7.7 \mathrm{~Hz}, 2 \mathrm{H}), 7.34-7.28(\mathrm{~m}$, 1H), $7.09(\mathrm{~d}, J=7.9 \mathrm{~Hz}, 2 \mathrm{H}), 6.91(\mathrm{~s}, 2 \mathrm{H}), 4.06(\mathrm{~s}, 2 \mathrm{H}), 2.31(\mathrm{~s}, 3 \mathrm{H}), 2.25(\mathrm{~s}, 6 \mathrm{H}) .{ }^{13} \mathrm{C}$ NMR (125 MHz, Chloroform-d) $\delta$ 141.0, 139.2, 138.6, 137.0, 135.7, 133.7, 128.9, 128.7, 128.2, 127.1, 127.00, 126.9, 34.3, 20.9, 20.2. HRMS (EI-QTOF): calcd for $\mathrm{C}_{22} \mathrm{H}_{22} 286.1722$, found 286.1729.

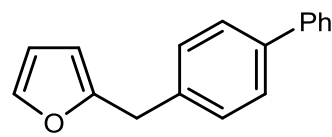

14

2-([1,1'-biphenyl]-4-ylmethyl)furan (14): According to the general procedure, 2(furan-2-yl)ethan-1-ol (44.8 mg, 0.4 mmol, 2.0 equiv.), 4-bromo-1,1'-biphenyl (46.6 mg, 0.2 mmol, 1.0 equiv.), $\mathrm{Ni}(\mathrm{dMebpy})\left(\mathrm{H}_{2} \mathrm{O}\right)_{4} \mathrm{Cl}_{2}(0.8 \mathrm{mg}, 0.002 \mathrm{mmol}, 0.01$ equiv.), DPA (0.7 mg, 0.002 mmol, 0.01 equiv.), $\mathbf{L 4}$ (20.0 mg, 0.07 mmol, 0.35 equiv.), $\mathrm{CeCl}_{3}$ (4.9 mg, 0.02 mmol, 0.1 equiv.), $\mathrm{Na}_{3} \mathrm{PO}_{4}$ (98.4 mg, 0.6 mmol, 3 equiv.), $\mathrm{CH}_{3} \mathrm{CN}$ (2 mL) and DMSO $(0.25 \mathrm{~mL})$ were used. After $36 \mathrm{~h}$, the product was isolated by flash chromatography as a white solid (40 mg, 85\%). ${ }^{1}$ H NMR (500 MHz, Chloroform-d) $\delta 7.57(\mathrm{~d}, J=7.7 \mathrm{~Hz}, 2 \mathrm{H}), 7.53(\mathrm{~d}, J=7.8 \mathrm{~Hz}, 2 \mathrm{H}), 7.43(\mathrm{t}, J=7.5 \mathrm{~Hz}, 2 \mathrm{H}), 7.32(\mathrm{dd}$, $J=16.3,8.0 \mathrm{~Hz}, 4 \mathrm{H}), 6.31(\mathrm{dd}, J=3.4,1.8 \mathrm{~Hz}, 1 \mathrm{H}), 6.05(\mathrm{~d}, J=3.1 \mathrm{~Hz}, 1 \mathrm{H}), 4.01(\mathrm{~s}$, 2H). ${ }^{13}$ C NMR (125 MHz, Chloroform-d) $\delta$ 154.4, 141.6, 140.9, 139.5, 137.2, 129.1, 
128.7, 127.3, 127.1, 127.0, 110.3, 106.3, 34.1. HRMS (EI-QTOF): calcd for $\mathrm{C}_{17} \mathrm{H}_{14} \mathrm{O}$ 234.1045, found 234.1051.

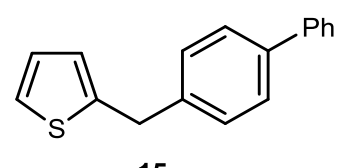

15

2-([1,1'-biphenyl]-4-ylmethyl)thiophene (15): According to the general procedure, 2(thiophen-2-yl)ethan-1-ol (51.3 mg, 0.4 mmol, 2.0 equiv.), 4-bromo-1,1'-biphenyl (46.6 mg, 0.2 mmol, 1.0 equiv.), $\mathrm{Ni}(\mathrm{dMebpy})\left(\mathrm{H}_{2} \mathrm{O}\right)_{4} \mathrm{Cl}_{2}$ (3.7 mg, $0.01 \mathrm{mmol}, 0.05$ equiv.), DPA (0.7 mg, $0.002 \mathrm{mmol}, 0.01$ equiv.), $\mathbf{L 4}$ (20.0 mg, $0.07 \mathrm{mmol}, 0.35$ equiv.), $\mathrm{CeCl}_{3}$ (4.9 mg, 0.02 mmol, 0.1 equiv.), $\mathrm{Na}_{3} \mathrm{PO}_{4}$ (98.4 mg, 0.6 mmol, 3 equiv.), $\mathrm{CH}_{3} \mathrm{CN}$ (2 mL) and DMSO $(0.25 \mathrm{~mL})$ were used. After $36 \mathrm{~h}$, the product was isolated by flash chromatography as a white solid (43 mg, 86\%). ${ }^{1}$ H NMR (500 MHz, Chloroform-d) $\delta 7.58(\mathrm{~d}, J=7.6 \mathrm{~Hz}, 2 \mathrm{H}), 7.54(\mathrm{~d}, J=7.8 \mathrm{~Hz}, 2 \mathrm{H}), 7.43(\mathrm{t}, J=7.5 \mathrm{~Hz}, 2 \mathrm{H}), 7.32(\mathrm{~d}, J$ $=8.0 \mathrm{~Hz}, 3 \mathrm{H}), 7.16(\mathrm{~d}, J=5.1 \mathrm{~Hz}, 1 \mathrm{H}), 6.94(\mathrm{t}, J=4.3 \mathrm{~Hz}, 1 \mathrm{H}), 6.84(\mathrm{~d}, J=3.4 \mathrm{~Hz}$, 1H), 4.20 (s, 2H). ${ }^{13}$ C NMR (125 MHz, Chloroform-d) $\delta$ 143.8, 140.9, 139.5, 139.4, 129.0, 128.7 127.3, 127.1, 127.0, 126.8, 125.2, 124.0, 35.6. HRMS (EI-QTOF): calcd for $\mathrm{C}_{17} \mathrm{H}_{14} \mathrm{~S} 250.0816$, found 250.0813 .

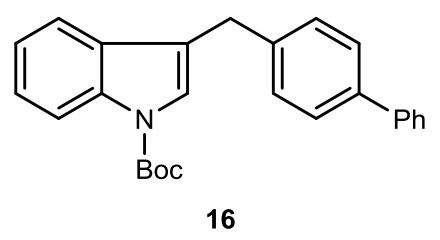

tert-butyl 3-([1,1'-biphenyl]-4-ylmethyl)-1H-indole-1-carboxylate (16): According to the general procedure, tert-butyl 3-(2-hydroxyethyl)-1H-indole-1-carboxylate (104.4 mg, 0.4 mmol, 2.0 equiv.), 4-bromo-1,1'-biphenyl (46.6 mg, $0.2 \mathrm{mmol}, 1.0$ equiv.), $\mathrm{Ni}(\mathrm{dMebpy})\left(\mathrm{H}_{2} \mathrm{O}\right)_{4} \mathrm{Cl}_{2}(0.8 \mathrm{mg}, 0.002 \mathrm{mmol}, 0.01$ equiv. $)$, DPA (0.7 mg, $0.002 \mathrm{mmol}$, 0.01 equiv.), $\mathbf{L} 4$ (20.0 mg, $0.07 \mathrm{mmol}, 0.35$ equiv.), $\mathrm{CeCl}_{3}$ (4.9 mg, $0.02 \mathrm{mmol}, 0.1$ equiv.), $\mathrm{Na}_{3} \mathrm{PO}_{4}$ (98.4 mg, $0.6 \mathrm{mmol}, 3$ equiv.), $\mathrm{CH}_{3} \mathrm{CN}(2 \mathrm{~mL})$ and DMSO (0.25 mL) were used. After $36 \mathrm{~h}$, the product was isolated by flash chromatography as a white 
solid (57 mg, 74\%). ${ }^{1}$ H NMR (500 MHz, Chloroform-d) $\delta 8.13$ (s, 1H), 7.58 (d, $J=$ $7.7 \mathrm{~Hz}, 2 \mathrm{H}), 7.53(\mathrm{~d}, J=7.8 \mathrm{~Hz}, 2 \mathrm{H}), 7.44(\mathrm{~m}, 4 \mathrm{H}), 7.34(\mathrm{dd}, J=16.3,7.9 \mathrm{~Hz}, 4 \mathrm{H})$, $7.21(\mathrm{t}, J=7.5 \mathrm{~Hz}, 1 \mathrm{H}), 4.09$ (s, 2H), 1.67 (s, 9H). ${ }^{13} \mathrm{C}$ NMR (125 MHz, Chloroformd) $\delta 150.2,140.9,139.1,138.8,130.5,129.0,128.7,127.2,127.1,127.0,124.3,123.6$, $122.4,119.9,119.4,115.2,83.3,31.0,28.2$.

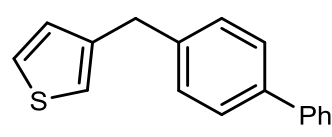

17

3-([1,1'-biphenyl]-4-ylmethyl)thiophene (17): According to the general procedure, 2(thiophen-3-yl)ethan-1-ol (51.3 mg, 0.4 mmol, 2.0 equiv.), 4-bromo-1,1'-biphenyl (46.6 mg, 0.2 mmol, 1.0 equiv.), $\mathrm{Ni}(\mathrm{dMebpy})\left(\mathrm{H}_{2} \mathrm{O}\right)_{4} \mathrm{Cl}_{2}(0.8 \mathrm{mg}, 0.002 \mathrm{mmol}, 0.01$ equiv.), DPA (0.7 mg, 0.002 mmol, 0.01 equiv.), $\mathbf{L 4}$ (20.0 mg, 0.07 mmol, 0.35 equiv.), $\mathrm{CeCl}_{3}$ (4.9 mg, 0.02 mmol, 0.1 equiv.), $\mathrm{Na}_{3} \mathrm{PO}_{4}$ (98.4 mg, 0.6 mmol, 3 equiv.), $\mathrm{CH}_{3} \mathrm{CN}$ (2 mL) and DMSO $(0.25 \mathrm{~mL})$ were used. After $36 \mathrm{~h}$, the product was isolated by flash chromatography as a white solid (45 mg, 90\%). ${ }^{1}$ H NMR (500 MHz, Chloroform-d) $\delta 7.60-7.55(\mathrm{~m}, 2 \mathrm{H}), 7.55-7.50(\mathrm{~m}, 2 \mathrm{H}), 7.45-7.39(\mathrm{~m}, 2 \mathrm{H}), 7.36-7.30(\mathrm{~m}, 1 \mathrm{H})$, $7.27(\mathrm{dd}, J=7.7,4.2 \mathrm{~Hz}, 3 \mathrm{H}), 6.96(\mathrm{dd}, J=9.4,3.9 \mathrm{~Hz}, 2 \mathrm{H}), 4.02(\mathrm{~s}, 2 \mathrm{H}) .{ }^{13} \mathbf{C} \mathbf{N M R}$ (125 MHz, Chloroform-d) $\delta$ 141.3, 141.0, 139.7, 139.1, 129.1, 128.7, 128.4, 127.2, 127.1, 127.0, 125.7, 121.3, 36.1. HRMS (EI-QTOF): calcd for $\mathrm{C}_{17} \mathrm{H}_{14} \mathrm{~S} 250.0816$, found 250.0816 .

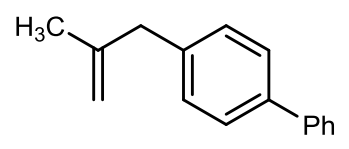

18

4-(2-methylallyl)-1,1'-biphenyl (18): According to the general procedure, 3methylbut-3-en-1-ol (34.4 mg, 0.4 mmol, 2.0 equiv.), 4-bromo-1,1'-biphenyl (46.6 mg, 0.2 mmol, 1.0 equiv.), $\mathrm{Ni}(\mathrm{dMebpy})\left(\mathrm{H}_{2} \mathrm{O}\right)_{4} \mathrm{Cl}_{2}(0.8 \mathrm{mg}, 0.002 \mathrm{mmol}, 0.01$ equiv.), DPA (0.7 mg, 0.002 mmol, 0.01 equiv.), $\mathbf{L} 4$ (20.0 mg, 0.07 mmol, 0.35 equiv.), $\mathrm{CeCl}_{3}$ (4.9 
mg, 0.02 mmol, 0.1 equiv.), $\mathrm{Na}_{3} \mathrm{PO}_{4}$ (98.4 mg, $0.6 \mathrm{mmol}, 3$ equiv.), $\mathrm{CH}_{3} \mathrm{CN}$ (2 mL) and DMSO $(0.25 \mathrm{~mL})$ were used. After $36 \mathrm{~h}$, the product was isolated by flash chromatography as a colorless oil (29 mg, 70\%). ${ }^{1} \mathbf{H}$ NMR (500 MHz, Chloroform- $\boldsymbol{d}$ ) $\delta 7.59(\mathrm{~d}, J=7.5 \mathrm{~Hz}, 2 \mathrm{H}), 7.55-7.50(\mathrm{~m}, 2 \mathrm{H}), 7.43(\mathrm{t}, J=7.6 \mathrm{~Hz}, 2 \mathrm{H}), 7.34(\mathrm{~d}, J=$ $7.6 \mathrm{~Hz}, 1 \mathrm{H}), 7.28-7.25(\mathrm{~m}, 2 \mathrm{H}), 4.81$ (d, $J=31.2 \mathrm{~Hz}, 2 \mathrm{H}), 3.36(\mathrm{~s}, 2 \mathrm{H}), 1.72(\mathrm{~s}, 3 \mathrm{H})$. ${ }^{13}$ C NMR (125 MHz, Chloroform-d) $\delta$ 145.1, 141.1, 139.0, 138.9, 129.3, 128.7, 127.1, 127.0, 112.1, 44.3, 22.1. HRMS (EI-QTOF): calcd for $\mathrm{C}_{16} \mathrm{H}_{16}$ 208.1252, found 208.1256.

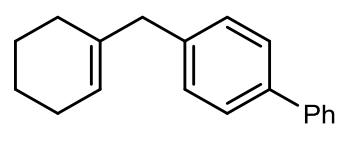

19

4-(cyclohex-1-en-1-ylmethyl)-1,1'-biphenyl (19): According to the general procedure, 2-(cyclohex-1-en-1-yl)ethan-1-ol (50.4 mg, 0.4 mmol, 2.0 equiv.), 4-bromo-1,1'biphenyl (46.6 mg, $0.2 \mathrm{mmol}, 1.0$ equiv.), $\mathrm{Ni}(\mathrm{dMebpy})\left(\mathrm{H}_{2} \mathrm{O}\right)_{4} \mathrm{Cl}_{2}(0.8 \mathrm{mg}, 0.002 \mathrm{mmol}$, 0.01 equiv.), DPA (0.7 mg, $0.002 \mathrm{mmol}, 0.01$ equiv.), $\mathbf{L} 4$ (20.0 mg, $0.07 \mathrm{mmol}, 0.35$ equiv.), $\mathrm{CeCl}_{3}$ (4.9 mg, $0.02 \mathrm{mmol}, 0.1$ equiv.), $\mathrm{Na}_{3} \mathrm{PO}_{4}$ (98.4 mg, $0.6 \mathrm{mmol}, 3$ equiv.), $\mathrm{CH}_{3} \mathrm{CN}(2 \mathrm{~mL})$ and DMSO $(0.25 \mathrm{~mL})$ were used. After $36 \mathrm{~h}$, the product was isolated by flash chromatography as a colorless oil (33 mg, 67\%). ${ }^{1} \mathbf{H}$ NMR (500 MHz, Chloroform-d) $\delta 7.51(\mathrm{~d}, J=7.5 \mathrm{~Hz}, 2 \mathrm{H}), 7.44(\mathrm{~d}, J=7.7 \mathrm{~Hz}, 2 \mathrm{H}), 7.35(\mathrm{t}, J=7.6 \mathrm{~Hz}$, 2H), $7.28-7.22(\mathrm{~m}, 1 \mathrm{H}), 7.20-7.13(\mathrm{~m}, 2 \mathrm{H}), 5.43(\mathrm{td}, J=3.8,2.1 \mathrm{~Hz}, 1 \mathrm{H}), 3.21(\mathrm{~s}$, 2H), 1.97 (m, 2H), 1.82 (m, 2H), 1.49 (m, 4H). ${ }^{13}$ C NMR (100 MHz, Chloroform-d) $\delta 141.1,139.6,138.8,137.1,129.3,128.7,127.0,126.9,123.1,44.3,28.1,25.4,22.9$, 22.4. HRMS (EI-QTOF): calcd for $\mathrm{C}_{19} \mathrm{H}_{20} 248.1565$, found 248.1560 .

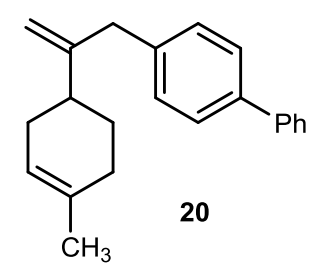


4-(2-(4-methylcyclohex-3-en-1-yl)allyl)-1,1'-biphenyl (20): According to the general procedure, 3-(4-methylcyclohex-3-en-1-yl)but-3-en-1-ol (66.4 mg, 0.4 mmol, 2.0 equiv.), 4-bromo-1,1'-biphenyl (46.6 mg, $0.2 \mathrm{mmol}, 1.0$ equiv.), $\mathrm{Ni}(\mathrm{dMebpy})\left(\mathrm{H}_{2} \mathrm{O}\right)_{4} \mathrm{Cl}_{2}$ (0.8 mg, 0.002 mmol, 0.01 equiv.), DPA ( $0.7 \mathrm{mg}, 0.002 \mathrm{mmol}, 0.01$ equiv.), L4 (20.0 mg, 0.07 mmol, 0.35 equiv.), $\mathrm{CeCl}_{3}$ (4.9 mg, 0.02 mmol, 0.1 equiv.), $\mathrm{Na}_{3} \mathrm{PO}_{4}$ (98.4 mg, $0.6 \mathrm{mmol}, 3$ equiv.), $\mathrm{CH}_{3} \mathrm{CN}(2 \mathrm{~mL})$ and DMSO $(0.25 \mathrm{~mL})$ were used. After $36 \mathrm{~h}$, the product was isolated by flash chromatography as a white solid (32 mg, 56\%). ${ }^{1} \mathbf{H}$ NMR (500 MHz, Chloroform-d) $\delta 7.52(\mathrm{~m}, 2 \mathrm{H}), 7.48-7.41(\mathrm{~m}, 2 \mathrm{H}), 7.36(\mathrm{~m}, 2 \mathrm{H}), 7.26(\mathrm{~m}$, 1H), $7.18(\mathrm{~m}, 2 \mathrm{H}), 5.31(\mathrm{~s}, 1 \mathrm{H}), 4.75(\mathrm{~d}, J=73.1 \mathrm{~Hz}, 2 \mathrm{H}), 3.36(\mathrm{~s}, 2 \mathrm{H}), 2.04(\mathrm{~m}, 2 \mathrm{H})$, $1.96-1.80(\mathrm{~m}, 3 \mathrm{H}), 1.80-1.72(\mathrm{~m}, 1 \mathrm{H}), 1.44(\mathrm{~m}, 1 \mathrm{H}) .{ }^{13} \mathrm{C}$ NMR (125 MHz, Chloroform- $d) \delta 153.5,141.0,139.2,138.9,133.8,129.5,128.7,127.0,120.6,110.0$, 41.6, 38.8, 31.4, 30.6, 28.3, 23.4. HRMS (EI-QTOF): calcd for $\mathrm{C}_{22} \mathrm{H}_{24}$ 288.1878, found 288.1882 .

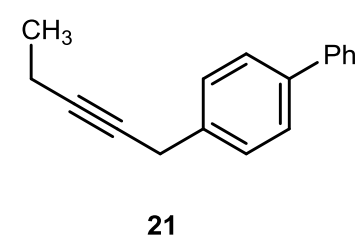

4-(pent-2-yn-1-yl)-1,1'-biphenyl (21): According to the general procedure, hex-3-yn1-ol (39.3 mg, 0.4 mmol, 2.0 equiv.), 4-bromo-1,1'-biphenyl (46.6 mg, 0.2 mmol, 1.0 equiv.), $\mathrm{Ni}(\mathrm{dMebpy})\left(\mathrm{H}_{2} \mathrm{O}\right)_{4} \mathrm{Cl}_{2}(3.7 \mathrm{mg}, 0.01 \mathrm{mmol}, 0.05$ equiv.), DPA (0.7 mg, 0.002 mmol, 0.01 equiv.), $\mathbf{L 4}$ (20.0 mg, 0.07 mmol, 0.35 equiv.), $\mathrm{CeCl}_{3}$ (4.9 mg, $0.02 \mathrm{mmol}$, 0.1 equiv.), $\mathrm{Na}_{3} \mathrm{PO}_{4}$ (98.4 mg, $0.6 \mathrm{mmol}, 3$ equiv.), $\mathrm{CH}_{3} \mathrm{CN}$ (2 mL) and DMSO (0.25 $\mathrm{mL}$ ) were used. After $36 \mathrm{~h}$, the product was isolated by flash chromatography as a colorless oil (24 mg, 55\%). ${ }^{1} \mathbf{H}$ NMR (400 MHz, Chloroform-d) $\delta 7.49$ (dd, $J=14.7$, $7.5 \mathrm{~Hz}, 4 \mathrm{H}), 7.35(\mathrm{~d}, J=7.8 \mathrm{~Hz}, 4 \mathrm{H}), 7.26(\mathrm{t}, J=7.4 \mathrm{~Hz}, 1 \mathrm{H}), 3.54(\mathrm{~d}, J=2.6 \mathrm{~Hz}, 2 \mathrm{H})$, $2.33-2.08(\mathrm{~m}, 2 \mathrm{H}), 1.11(\mathrm{t}, J=7.5 \mathrm{~Hz}, 3 \mathrm{H}) .{ }^{13} \mathrm{C}$ NMR (125 MHz, Chloroform-d) $\delta$ 141.0, 139.4, 136.7, 128.7, 128.3, 127.2, 127.1, 127.0, 84.1, 76.8, 24.8, 14.2, 12.5. HRMS (EI-QTOF): calcd for $\mathrm{C}_{17} \mathrm{H}_{16} 220.1252$, found 220.1257 . 


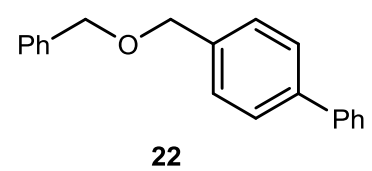

4-((benzyloxy)methyl)-1,1'-biphenyl (22): According to the general procedure, 2(benzyloxy)ethan-1-ol (60.9 mg, 0.4 mmol, 2.0 equiv.), 4-bromo-1,1'-biphenyl (46.6 mg, 0.2 mmol, 1.0 equiv.), $\mathrm{Ni}(\mathrm{dMebpy})\left(\mathrm{H}_{2} \mathrm{O}\right)_{4} \mathrm{Cl}_{2}(0.8 \mathrm{mg}, 0.002 \mathrm{mmol}, 0.01$ equiv.), DPA (0.7 mg, 0.002 mmol, 0.01 equiv.), $\mathbf{L 4}$ (20.0 mg, 0.07 mmol, 0.35 equiv.), $\mathrm{CeCl}_{3}$ (4.9 mg, 0.02 mmol, 0.1 equiv.), $\mathrm{Na}_{3} \mathrm{PO}_{4}$ ( $98.4 \mathrm{mg}, 0.6 \mathrm{mmol}, 3$ equiv.), $\mathrm{CH}_{3} \mathrm{CN}$ (2 mL) and DMSO $(0.25 \mathrm{~mL})$ were used. After $36 \mathrm{~h}$, the product was isolated by flash chromatography as a colorless oil (28 mg, 51\%). ${ }^{1} \mathbf{H}$ NMR (500 MHz, Chloroform- $d$ ) $\delta 7.59$ (dd, $J=8.1,3.4 \mathrm{~Hz}, 4 \mathrm{H}), 7.44$ (t, $J=7.2 \mathrm{~Hz}, 4 \mathrm{H}), 7.36(\mathrm{~m}, 6 \mathrm{H}), 4.60$ (s, 4H). ${ }^{13}$ C NMR (125 MHz, Chloroform-d) $\delta$ 140.9, 140.6, 138.2, 137.3, 128.8, 128.4, 128.2, 127.8, 127.7, 127.3, 127.2, 127.1, 72.2, 71.8. HRMS (EI-QTOF): calcd for $\mathrm{C}_{20} \mathrm{H}_{18} \mathrm{O}$ 274.1358, found 274.1363.

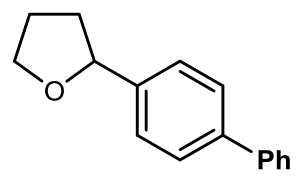

23

2-([1,1'-biphenyl]-4-yl)tetrahydrofuran (23): According to the general procedure, (tetrahydrofuran-2-yl)methanol (40.8 mg, $0.4 \mathrm{mmol}, 2.0$ equiv.), 4-bromo-1,1'biphenyl (46.6 mg, $0.2 \mathrm{mmol}, 1.0$ equiv.), $\mathrm{Ni}(\mathrm{dMebpy})\left(\mathrm{H}_{2} \mathrm{O}\right)_{4} \mathrm{Cl}_{2}(0.8 \mathrm{mg}, 0.002 \mathrm{mmol}$, 0.01 equiv.), DPA (0.7 mg, $0.002 \mathrm{mmol}, 0.01$ equiv.), L4 (20.0 mg, $0.07 \mathrm{mmol}, 0.35$ equiv.), $\mathrm{CeCl}_{3}$ (4.9 mg, $0.02 \mathrm{mmol}, 0.1$ equiv.), $\mathrm{Na}_{3} \mathrm{PO}_{4}$ (98.4 mg, $0.6 \mathrm{mmol}, 3$ equiv.), $\mathrm{CH}_{3} \mathrm{CN}(2 \mathrm{~mL})$ and DMSO $(0.25 \mathrm{~mL})$ were used. After $36 \mathrm{~h}$, the product was isolated by flash chromatography as a colorless oil (25 mg, 56\%). ${ }^{1} \mathbf{H}$ NMR (500 MHz, Chloroform-d) $\delta 7.65-7.51(\mathrm{~m}, 4 \mathrm{H}), 7.51-7.37(\mathrm{~m}, 4 \mathrm{H}), 7.37-7.27(\mathrm{~m}, 1 \mathrm{H}), 4.94$ $(\mathrm{td}, J=7.3,2.3 \mathrm{~Hz}, 1 \mathrm{H}), 4.18-4.05(\mathrm{~m}, 1 \mathrm{H}), 4.00-3.85(\mathrm{~m}, 1 \mathrm{H}), 2.35(\mathrm{~m}, 1 \mathrm{H}), 2.03$ (m, 2H), 1.86 (m, 1H). ${ }^{13}$ C NMR (125 MHz, Chloroform-d) $\delta$ 142.5, 141.0, 140.1, 
128.7, 127.1, 126.1, 80.4, 68.7, 34.6, 26.1. HRMS (EI-QTOF): calcd for $\mathrm{C}_{16} \mathrm{H}_{16} \mathrm{O}$ 224.1201, found 224.1197.

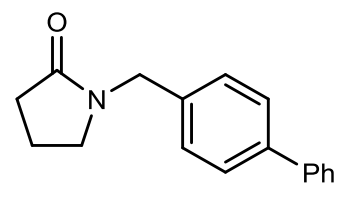

24

1-([1,1'-biphenyl]-4-ylmethyl)pyrrolidin-2-one (24): According to the general procedure, 1-(2-hydroxyethyl)pyrrolidin-2-one (51.7 mg, $0.4 \mathrm{mmol}, 2.0$ equiv.), 4bromo-1,1'-biphenyl (46.6 mg, $0.2 \mathrm{mmol}, 1.0$ equiv.), $\mathrm{Ni}(\mathrm{dMebpy})\left(\mathrm{H}_{2} \mathrm{O}\right)_{4} \mathrm{Cl}_{2}(0.8 \mathrm{mg}$, $0.002 \mathrm{mmol}, 0.01$ equiv.), DPA (0.7 mg, $0.002 \mathrm{mmol}, 0.01$ equiv.), L4 (20.0 mg, 0.07 mmol, 0.35 equiv.), $\mathrm{CeCl}_{3}$ (4.9 mg, 0.02 mmol, 0.1 equiv.), $\mathrm{Na}_{3} \mathrm{PO}_{4}$ (98.4 mg, $0.6 \mathrm{mmol}$, 3 equiv.), $\mathrm{CH}_{3} \mathrm{CN}(2 \mathrm{~mL})$ and DMSO $(0.25 \mathrm{~mL})$ were used. After $36 \mathrm{~h}$, the product was isolated by flash chromatography as a white solid (37 mg, 74\%). ${ }^{\mathbf{1}} \mathbf{H}$ NMR (500 MHz, Chloroform-d) $\delta 7.57(\mathrm{dd}, J=10.2,7.7 \mathrm{~Hz}, 4 \mathrm{H}), 7.43(\mathrm{t}, J=7.6 \mathrm{~Hz}, 2 \mathrm{H}), 7.33(\mathrm{dd}, J$ $=17.6,7.7 \mathrm{~Hz}, 3 \mathrm{H}), 4.49(\mathrm{~s}, 2 \mathrm{H}), 3.30(\mathrm{t}, J=7.0 \mathrm{~Hz}, 2 \mathrm{H}), 2.46(\mathrm{t}, J=8.1 \mathrm{~Hz}, 2 \mathrm{H}), 2.01$ (q, $J=7.6 \mathrm{~Hz}, 2 \mathrm{H}) .{ }^{13} \mathbf{C}$ NMR (125 MHz, Chloroform-d) $\delta$ 174.9, 140.6, 140.5, 135.6, 128.7, 128.5, 127.3, 127.0, 46.6, 46.2, 30.9, 17.7. HRMS (ESI+): calcd for $\mathrm{C}_{17} \mathrm{H}_{18} \mathrm{NO}$ $(\mathrm{M}+\mathrm{H}) 252.1383$, found 252.1383.

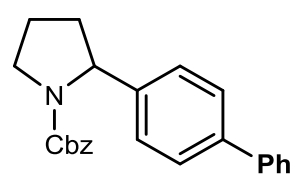

25

benzyl 2-([1,1'-biphenyl]-4-yl)pyrrolidine-1-carboxylate (25): According to the general procedure, benzyl (S)-2-(hydroxymethyl)pyrrolidine-1-carboxylate (94.1 mg, 0.4 mmol, 2.0 equiv.), 4-bromo-1,1'-biphenyl (46.6 mg, $0.2 \mathrm{mmol}, 1.0$ equiv.), $\mathrm{Ni}(\mathrm{dMebpy})\left(\mathrm{H}_{2} \mathrm{O}\right)_{4} \mathrm{Cl}_{2}(0.8 \mathrm{mg}, 0.002 \mathrm{mmol}, 0.01$ equiv.), DPA (0.7 mg, $0.002 \mathrm{mmol}$, 0.01 equiv.), $\mathbf{L} 4$ (20.0 mg, $0.07 \mathrm{mmol}, 0.35$ equiv.), $\mathrm{CeCl}_{3}$ (4.9 mg, $0.02 \mathrm{mmol}, 0.1$ equiv.), $\mathrm{Na}_{3} \mathrm{PO}_{4}$ (98.4 mg, $0.6 \mathrm{mmol}, 3$ equiv.), $\mathrm{CH}_{3} \mathrm{CN}(2 \mathrm{~mL})$ and DMSO (0.25 mL) 
were used. After $36 \mathrm{~h}$, the product was isolated by flash chromatography as a colorless oil (47 mg, 66\%). ${ }^{1} \mathbf{H}$ NMR (500 MHz, Chloroform-d) $\delta 7.49$ (dd, J = 35.4, 7.8 Hz, 4H), $7.41-7.00(\mathrm{~m}, 9 \mathrm{H}), 6.83(\mathrm{~d}, \mathrm{~J}=7.1 \mathrm{~Hz}, 1 \mathrm{H}), 5.26-4.58(\mathrm{~m}, 3 \mathrm{H}), 3.73-3.50(\mathrm{~m}$, 2H), 2.39 - $2.16(\mathrm{~m}, 1 \mathrm{H}), 1.86(\mathrm{~m}, 3 \mathrm{H}) .{ }^{13} \mathrm{C}$ NMR (125 MHz, Chloroform-d) $\delta$ 155.0, $154.9,143.4,142.6,140.9,139.7,137.2,136.7,128.8,128.7,128.4,128.1,127.9,127.5$, 127.2, 127.1, 127.0, 126.0, 66.8, 66.6, 61.1, 60.8, 47.6, 47.2, 35.9, 34.8, 23.7, 23.0. ${ }^{13} \mathbf{C}$ NMR (125 MHz, Acetone-d6) $\delta$ 155.2, 145.1\&144.4, 141.7, 140.2, 138.5\&138.2, $129.7,129.2,128.9,128.6 \& 128.5,128.1 \& 128.0,127.9,127.6,127.0,66.9 \& 66.6$, 61.8\&61.6, 48.4\&47.8, 36.6\&35.4, 24.2\&23.6. HRMS (ESI+): calcd for $\mathrm{C}_{24} \mathrm{H}_{24} \mathrm{NO}_{2}$ $(\mathrm{M}+\mathrm{H}) 358.1802$, found 358.1805.

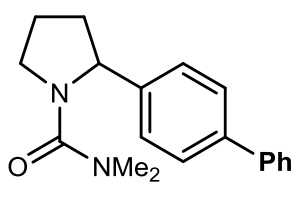

26

2-([1,1'-biphenyl]-4-yl)-N,N-dimethylpyrrolidine-1-carboxamide (26): According to the general procedure, (S)-2-(hydroxymethyl)-N,N-dimethylpyrrolidine-1carboxamide (68.8 mg, 0.4 mmol, 2.0 equiv.), 4-bromo-1,1'-biphenyl (46.6 mg, 0.2 mmol, 1.0 equiv.), $\mathrm{Ni}(\mathrm{dMebpy})\left(\mathrm{H}_{2} \mathrm{O}\right)_{4} \mathrm{Cl}_{2}(0.8 \mathrm{mg}, 0.002 \mathrm{mmol}, 0.01$ equiv.), DPA (0.7 mg, 0.002 mmol, 0.01 equiv.), $\mathbf{L 4}$ (20.0 mg, 0.07 mmol, 0.35 equiv.), $\mathrm{CeCl}_{3}$ (4.9 mg, 0.02 mmol, 0.1 equiv.), $\mathrm{Na}_{3} \mathrm{PO}_{4}$ (98.4 mg, $0.6 \mathrm{mmol}, 3$ equiv.), $\mathrm{CH}_{3} \mathrm{CN}$ (2 mL) and DMSO $(0.25 \mathrm{~mL})$ were used. After $36 \mathrm{~h}$, the product was isolated by flash chromatography as a white solid (35 mg, 60\%). ${ }^{1}$ H NMR (500 MHz, Chloroform-d) $\delta 7.61-7.56(\mathrm{~m}, 2 \mathrm{H}), 7.54(\mathrm{~d}, J=7.9 \mathrm{~Hz}, 2 \mathrm{H}), 7.44(\mathrm{t}, J=7.5 \mathrm{~Hz}, 2 \mathrm{H}), 7.38(\mathrm{~d}, J=$ $7.9 \mathrm{~Hz}, 2 \mathrm{H}), 7.34$ (t, J=7.3 Hz, 1H), $5.08(\mathrm{~m}, 1 \mathrm{H}), 3.68(\mathrm{~m}, 1 \mathrm{H}), 3.57(\mathrm{~m}, 1 \mathrm{H}), 2.88(\mathrm{~s}$, 6H), $2.39(\mathrm{~m}, 1 \mathrm{H}), 2.01(\mathrm{~m}, 1 \mathrm{H}), 1.97-1.87(\mathrm{~m}, 1 \mathrm{H}), 1.82(\mathrm{~m}, 1 \mathrm{H}) .{ }^{13} \mathrm{C}$ NMR (100 MHz, Chloroform-d) $\delta$ 163.3, 143.5, 141.1, 139.6, 128.6, 127.1, 127.0, 126.9, 126.4, 62.3, 51.2, 38.0, 35.9, 25.8. ${ }^{13}$ C NMR (125 MHz, Acetone-d6) $\delta$ 163.5, 145.6, 141.9, 140.0, 129.7, 127.9, 127.6, 127.5, 62.8, 51.9, 38.1, 36.6, 26.6. HRMS (ESI+): calcd for $\mathrm{C}_{19} \mathrm{H}_{23} \mathrm{~N}_{2} \mathrm{O}(\mathrm{M}+\mathrm{H})$ 295.1805, found 295.1807. 


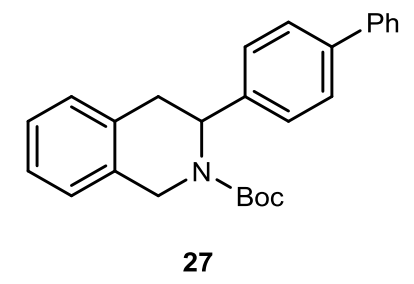

tert-butyl 3-([1,1'-biphenyl]-4-yl)-3,4-dihydroisoquinoline-2(1H)-carboxylate (27): According to the general procedure, tert-butyl (R)-3-(hydroxymethyl)-3,4dihydroisoquinoline-2(1H)-carboxylate (105.2 mg, $0.4 \mathrm{mmol}, 2.0$ equiv.), 4-bromo1,1'-biphenyl (46.6 mg, $0.2 \mathrm{mmol}, 1.0$ equiv.), $\mathrm{Ni}(\mathrm{dMebpy})\left(\mathrm{H}_{2} \mathrm{O}\right)_{4} \mathrm{Cl}_{2}(0.8 \mathrm{mg}, 0.002$ mmol, 0.01 equiv.), DPA (0.7 mg, $0.002 \mathrm{mmol}, 0.01$ equiv.), $\mathbf{L 4}$ (20.0 mg, $0.07 \mathrm{mmol}$, 0.35 equiv.), $\mathrm{CeCl}_{3}$ (4.9 mg, $0.02 \mathrm{mmol}, 0.1$ equiv.), $\mathrm{Na}_{3} \mathrm{PO}_{4}$ (98.4 mg, $0.6 \mathrm{mmol}, 3$ equiv.), $\mathrm{CH}_{3} \mathrm{CN}(2 \mathrm{~mL})$ and DMSO $(0.25 \mathrm{~mL})$ were used. After $36 \mathrm{~h}$, the product was isolated by flash chromatography as a colorless oil (43 mg, 56\%). ${ }^{1}$ H NMR (500 MHz, Chloroform-d) $\delta 7.46(\mathrm{~d}, J=7.6 \mathrm{~Hz}, 2 \mathrm{H}), 7.38(\mathrm{~d}, J=7.9 \mathrm{~Hz}, 2 \mathrm{H}), 7.33(\mathrm{t}, J=7.6 \mathrm{~Hz}$, 2H), $7.24(\mathrm{t}, J=7.4 \mathrm{~Hz}, 1 \mathrm{H}), 7.19(\mathrm{~s}, 1 \mathrm{H}), 7.15-7.09(\mathrm{~m}, 5 \mathrm{H}), 5.30(\mathrm{~s}, 1 \mathrm{H}), 4.80(\mathrm{~m}$, 1H), 4.24 (s, 1H), 3.35 - 3.20 (m, 1H), 3.07 (s, 1H), 1.34 (s, 9H). ${ }^{\mathbf{1 3}} \mathrm{C}$ NMR (125 MHz,

Chloroform- $d$, some aromatic signals could not be observed) $\delta 155.2,140.7,139.6,128.7,127.1,126.9,126.4,126.0,80.1,43.7,28.3 .{ }^{13} \mathbf{C}$ NMR (126 MHz, Acetone-d6) $\delta$ 155.5, 141.4, 140.2, 134.8, 129.7, 129.2, 128.1, 127.7, 127.6, 127.5, 127.2, 126.7, 80.1, 55.2, 44.5, 35.6, 28.5. HRMS (ESI+): calcd for $\mathrm{C}_{26} \mathrm{H}_{28} \mathrm{NO}_{2}$ $(\mathrm{M}+\mathrm{H}) 386.2120$, found 386.2122.

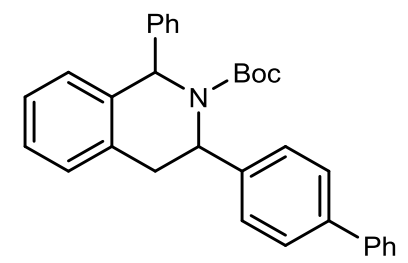

28

tert-butyl 3-([1,1'-biphenyl]-4-yl)-1-phenyl-3,4-dihydroisoquinoline-2(1H)carboxylate (28): According to the general procedure, tert-butyl (3R)-3(hydroxymethyl)-1-phenyl-3,4-dihydroisoquinoline-2(1H)-carboxylate (135.7 mg, 0.4 
mmol, 2.0 equiv.), 4-bromo-1,1'-biphenyl (46.6 mg, $0.2 \mathrm{mmol}, 1.0$ equiv.), $\mathrm{Ni}(\mathrm{dMebpy})\left(\mathrm{H}_{2} \mathrm{O}\right)_{4} \mathrm{Cl}_{2}(0.8 \mathrm{mg}, 0.002 \mathrm{mmol}, 0.01$ equiv.), DPA (0.7 mg, $0.002 \mathrm{mmol}$, 0.01 equiv.), $\mathbf{L 4}$ (20.0 mg, $0.07 \mathrm{mmol}, 0.35$ equiv.), $\mathrm{CeCl}_{3}$ (4.9 mg, $0.02 \mathrm{mmol}, 0.1$ equiv.), $\mathrm{Na}_{3} \mathrm{PO}_{4}$ (98.4 mg, $0.6 \mathrm{mmol}, 3$ equiv.), $\mathrm{CH}_{3} \mathrm{CN}(2 \mathrm{~mL})$ and DMSO (0.25 mL) were used. After $36 \mathrm{~h}$, the product was isolated by flash chromatography as a white solid (58 mg, 63\%, 2:1 d.r.). ${ }^{1}$ H NMR (400 MHz, Chloroform-d) $\delta 7.44$ (d, $J=7.8$ $\mathrm{Hz}, 3 \mathrm{H}), 7.29(\mathrm{~d}, J=7.0 \mathrm{~Hz}, 3 \mathrm{H}), 7.26-7.08(\mathrm{~m}, 6 \mathrm{H}), 7.20-6.86(\mathrm{~m}, 4 \mathrm{H}), 6.80(\mathrm{t}, J$ $=7.0 \mathrm{~Hz}, 2 \mathrm{H}), 6.35(\mathrm{~m}, 1 \mathrm{H}), 5.78-5.25(\mathrm{~m}, 1 \mathrm{H}), 3.52-3.12(\mathrm{~m}, 1 \mathrm{H}), 2.72(\mathrm{~m}, 1 \mathrm{H})$, $1.19(\mathrm{~m}, 4 \mathrm{H}), 1.11(\mathrm{~s}, 5 \mathrm{H}) \cdot{ }^{13} \mathrm{C}$ NMR (101 MHz, Chloroform- $d$, some aromatic signals could not be observed) $\delta 155.7,144.4,142.2,140.8,139.0,138.3,133.0,128.9$, $128.6,128.3,127.7,127.3,126.8,126.7,126.3,126.0,125.7,80.2,60.7 \& 58.8$, 57.1\&56.0, 36.1, 28.1. ${ }^{13}$ C NMR (101 MHz, Acetone-d6) $\delta$ 155.9, 146.3\&145.8, 144.5\&144.0, 141.5, 139.7\&139.6, 133.9\&133.2, 129.6, 129.1, 128.5, 128.2, 128.0, $127.7,127.5,127.4,127.1,126.9,126.6,80.1,61.9 \& 60.0,57.8 \& 56.4,36.8,28.3$. HRMS (ESI+): calcd for $\mathrm{C}_{32} \mathrm{H}_{31} \mathrm{NO}_{2} \mathrm{Na}(\mathrm{M}+\mathrm{Na})$ 484.2247, found 484.2257.

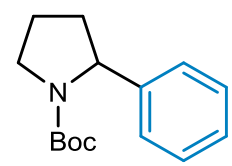

29

tert-butyl 2-phenylpyrrolidine-1-carboxylate (29): According to the general procedure, tert-butyl 2-(hydroxymethyl)pyrrolidine-1-carboxylate $(80.5 \mathrm{mg}, 0.4 \mathrm{mmol}$, 2.0 equiv.), bromobenzene (31.2 mg, $0.2 \mathrm{mmol}, 1.0$ equiv.), $\mathrm{Ni}(\mathrm{dMebpy})\left(\mathrm{H}_{2} \mathrm{O}\right)_{4} \mathrm{Cl}_{2}(0.8$ mg, 0.002 mmol, 0.01 equiv.), DPA (0.7 mg, $0.002 \mathrm{mmol}, 0.01$ equiv.), L4 (20.0 mg, 0.07 mmol, 0.35 equiv.), $\mathrm{CeCl}_{3}$ (4.9 mg, $0.02 \mathrm{mmol}, 0.1$ equiv.), $\mathrm{Na}_{3} \mathrm{PO}_{4}$ (98.4 mg, 0.6 mmol, 3 equiv.), $\mathrm{CH}_{3} \mathrm{CN}(2 \mathrm{~mL})$ and DMSO $(0.25 \mathrm{~mL})$ were used. After $36 \mathrm{~h}$, the product was isolated by flash chromatography as a colorless oil (37 mg, $74 \%) .{ }^{1} \mathbf{H}$ NMR (500 MHz, Chloroform-d) $\delta 7.29(\mathrm{t}, J=7.4 \mathrm{~Hz}, 2 \mathrm{H}), 7.23-7.11(\mathrm{~m}, 3 \mathrm{H}), 5.06-4.67$ (m, 1H), $3.63(\mathrm{~m}, 2 \mathrm{H}), 2.32(\mathrm{~m}, 1 \mathrm{H}), 1.87(\mathrm{~m}, 3 \mathrm{H}), 1.46(\mathrm{~s}, 3 \mathrm{H}), 1.17(\mathrm{~s}, 6 \mathrm{H}) .{ }^{13} \mathrm{C}$ NMR (125 MHz, Chloroform-d) $\delta$ 154.5, 145.1, 128.1, 126.4, 125.4, 79.1, 61.3, 60.7, 47.1, 
36.0, 28.1, 23.2. ${ }^{13} \mathbf{C}$ NMR (126 MHz, Acetone-d $) \delta$ 154.6, 146.6\&145.6, 128.9, 127.2, $126.4,79.0 \& 78.8,62.1 \& 61.4,47.8,36.7 \& 35.4,28.7 \& 28.3,24.0 \& 23.8$. HRMS (EIQTOF): calcd for $\mathrm{C}_{15} \mathrm{H}_{21} \mathrm{NO}_{2} 247.1572$, found 247.1571 .

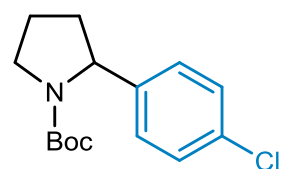

30

tert-butyl 2-(4-chlorophenyl)pyrrolidine-1-carboxylate (30): According to the general procedure, tert-butyl 2-(hydroxymethyl)pyrrolidine-1-carboxylate $(80.5 \mathrm{mg}$, $0.4 \mathrm{mmol}, 2.0$ equiv.), 1-bromo-4-chlorobenzene (38 mg, $0.2 \mathrm{mmol}, 1.0$ equiv.), $\mathrm{Ni}(\mathrm{dMebpy})\left(\mathrm{H}_{2} \mathrm{O}\right)_{4} \mathrm{Cl}_{2}(0.8 \mathrm{mg}, 0.002 \mathrm{mmol}, 0.01$ equiv. $)$, DPA (0.7 mg, $0.002 \mathrm{mmol}$, 0.01 equiv.), $\mathbf{L} 4$ (20.0 mg, $0.07 \mathrm{mmol}, 0.35$ equiv.), $\mathrm{CeCl}_{3}$ (4.9 mg, $0.02 \mathrm{mmol}, 0.1$ equiv.), $\mathrm{Na}_{3} \mathrm{PO}_{4}$ (98.4 mg, $0.6 \mathrm{mmol}, 3$ equiv.), $\mathrm{CH}_{3} \mathrm{CN}$ (2 mL) and DMSO (0.25 mL) were used. After $36 \mathrm{~h}$, the product was isolated by flash chromatography as a colorless oil (45 mg, 80\%). ${ }^{\mathbf{1} H}$ NMR (500 MHz, Chloroform- $\boldsymbol{d}$ ) $\delta 7.27$ (d, $\left.J=8.1 \mathrm{~Hz}, 2 \mathrm{H}\right), 7.10$ $(\mathrm{d}, J=8.0 \mathrm{~Hz}, 2 \mathrm{H}), 5.00-4.62(\mathrm{~m}, 1 \mathrm{H}), 3.69-3.44(\mathrm{~m}, 2 \mathrm{H}), 2.30(\mathrm{~m}, 1 \mathrm{H}), 1.94-1.74$ (m, 3H), 1.45 (s, 3H), 1.20 (s, 6H). ${ }^{13} \mathrm{C}$ NMR (125 MHz, Chloroform-d) $\delta$ 154.5, $143.7,132.1,128.4 \& 128.2,126.8,79.5,60.8 \& 60.2,47.3 \& 47.1,36.0 \& 34.8,28.5 \& 28.2$, 23.5\&23.1. HRMS (EI-QTOF): calcd for $\mathrm{C}_{15} \mathrm{H}_{20} \mathrm{ClNO}_{2} 281.1183$, found 281.1176.

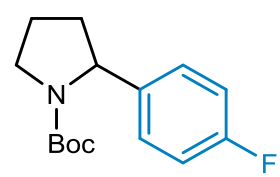

31

tert-butyl 2-(4-fluorophenyl)pyrrolidine-1-carboxylate (31): According to the general procedure, tert-butyl 2-(hydroxymethyl)pyrrolidine-1-carboxylate $(80.5 \mathrm{mg}$, $0.4 \mathrm{mmol}, 2.0$ equiv.), 1-bromo-4-fluorobenzene (34.8 mg, $0.2 \mathrm{mmol}, 1.0$ equiv.), $\mathrm{Ni}(\mathrm{dMebpy})\left(\mathrm{H}_{2} \mathrm{O}\right){ }_{4} \mathrm{Cl}_{2}(0.8 \mathrm{mg}, 0.002 \mathrm{mmol}, 0.01$ equiv.), DPA (0.7 mg, $0.002 \mathrm{mmol}$, 0.01 equiv.), $\mathbf{L} 4$ (20.0 mg, $0.07 \mathrm{mmol}, 0.35$ equiv.), $\mathrm{CeCl}_{3}$ (4.9 mg, $0.02 \mathrm{mmol}, 0.1$ 
equiv.), $\mathrm{Na}_{3} \mathrm{PO}_{4}$ (98.4 mg, $0.6 \mathrm{mmol}, 3$ equiv.), $\mathrm{CH}_{3} \mathrm{CN}(2 \mathrm{~mL})$ and DMSO (0.25 mL) were used. After $36 \mathrm{~h}$, the product was isolated by flash chromatography as a colorless oil (39 mg, 74\%). ${ }^{1} \mathbf{H}$ NMR (500 MHz, Chloroform-d) $\delta 7.13$ (dd, $J=8.3,5.3 \mathrm{~Hz}, 2 \mathrm{H}$ ), $6.98(\mathrm{t}, J=8.5 \mathrm{~Hz}, 2 \mathrm{H}), 5.12-4.57(\mathrm{~m}, 1 \mathrm{H}), 3.61(\mathrm{~m}, 2 \mathrm{H}), 2.31(\mathrm{~m}, 1 \mathrm{H}), 1.84(\mathrm{~m}, 3 \mathrm{H})$, $1.45(\mathrm{~s}, 3 \mathrm{H}), 1.19$ (s, 6H). ${ }^{13} \mathrm{C}$ NMR (125 MHz, Chloroform-d) $\delta 161.5(\mathrm{~d}, J=242.5$ Hz), 154.5, 140.8, 127.0 (d, $J=8.8 \mathrm{~Hz}), 114.9$ (d, $J=22.7 \mathrm{~Hz}), 79.4,60.7 \& 60.1$, 47.3\&47.1, 36.1\&34.9, 28.5\&28.1, 23.4\&23.1. ${ }^{19}$ F NMR (470 MHz, Chloroform-d) $\delta$-116.85, -117.07. HRMS (EI-QTOF): calcd for $\mathrm{C}_{15} \mathrm{H}_{20} \mathrm{FNO}_{2}$ 265.1478, found 265.1473 .

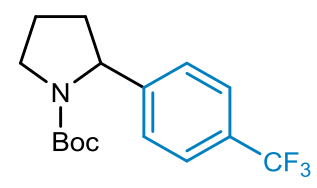

32

tert-butyl 2-(4-(trifluoromethyl)phenyl)pyrrolidine-1-carboxylate (32): According to the general procedure, tert-butyl 2-(hydroxymethyl)pyrrolidine-1-carboxylate $(80.5$ mg, 0.4 mmol, 2.0 equiv.), 1-bromo-4-(trifluoromethyl)benzene (44.8 mg, $0.2 \mathrm{mmol}$, 1.0 equiv. $), \mathrm{Ni}(\mathrm{dMebpy})\left(\mathrm{H}_{2} \mathrm{O}\right)_{4} \mathrm{Cl}_{2}(0.8 \mathrm{mg}, 0.002 \mathrm{mmol}, 0.01$ equiv. $)$, DPA (0.7 mg, 0.002 mmol, 0.01 equiv.), $\mathbf{L 4}$ (20.0 mg, $0.07 \mathrm{mmol}, 0.35$ equiv.), $\mathrm{CeCl}_{3}$ (4.9 mg, 0.02 mmol, 0.1 equiv.), $\mathrm{Na}_{3} \mathrm{PO}_{4}$ (98.4 mg, 0.6 mmol, 3 equiv.), $\mathrm{CH}_{3} \mathrm{CN}$ ( $2 \mathrm{~mL}$ ) and DMSO $(0.25 \mathrm{~mL})$ were used. After $36 \mathrm{~h}$, the product was isolated by flash chromatography as a colorless oil (39 mg, 62\%). ${ }^{1}$ H NMR (500 MHz, Chloroform-d) $\delta 7.49$ (d, $J=8.0$ $\mathrm{Hz}, 2 \mathrm{H}), 7.21(\mathrm{~d}, J=8.0 \mathrm{~Hz}, 2 \mathrm{H}), 4.82(\mathrm{~m}, 1 \mathrm{H}), 3.64-3.40(\mathrm{~m}, 2 \mathrm{H}), 2.33-2.21(\mathrm{~m}$, 1H), $1.77(\mathrm{~m}, 3 \mathrm{H}), 1.38(\mathrm{~s}, 3 \mathrm{H}), 1.11(\mathrm{~s}, 6 \mathrm{H}) .{ }^{13} \mathrm{C}$ NMR (125 MHz, Chloroform-d) $\delta$ 154.4, 149.5, 148.1, $128.9(\mathrm{q}, J=31.5 \mathrm{~Hz}), 125.8,125.3,125.2(\mathrm{q}, J=272.2 \mathrm{~Hz}), 115.8$, 79.6, 61.0\&60.5, 47.7\&47.1, 35.9\&34.6, 28.5\&28.1, 23.2. ${ }^{19}$ F NMR (470 MHz, Chloroform- $d$ ) $\delta-62.30,-62.34$. HRMS (EI-QTOF): calcd for $\mathrm{C}_{16} \mathrm{H}_{20} \mathrm{~F}_{3} \mathrm{NO}_{2}$ 315.1446, found 315.1447. 


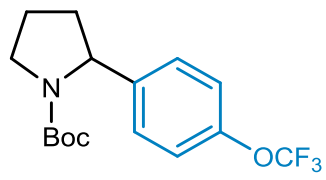

33

tert-butyl 2-(4-(trifluoromethoxy)phenyl)pyrrolidine-1-carboxylate

(33):

According to the general procedure, tert-butyl 2-(hydroxymethyl)pyrrolidine-1carboxylate (80.5 mg, $0.4 \mathrm{mmol}, 2.0$ equiv.), 1-bromo-4-(trifluoromethoxy)benzene (48.2 mg, $0.2 \mathrm{mmol}, 1.0$ equiv.), $\mathrm{Ni}(\mathrm{dMebpy})\left(\mathrm{H}_{2} \mathrm{O}\right)_{4} \mathrm{Cl}_{2}(0.8 \mathrm{mg}, 0.002 \mathrm{mmol}, 0.01$ equiv.), DPA (0.7 mg, $0.002 \mathrm{mmol}, 0.01$ equiv.), L4 (20.0 mg, $0.07 \mathrm{mmol}, 0.35$ equiv.), $\mathrm{CeCl}_{3}$ (4.9 mg, 0.02 mmol, 0.1 equiv.), $\mathrm{Na}_{3} \mathrm{PO}_{4}$ (98.4 mg, 0.6 mmol, 3 equiv.), $\mathrm{CH}_{3} \mathrm{CN}$ $(2 \mathrm{~mL})$ and DMSO $(0.25 \mathrm{~mL})$ were used. After $36 \mathrm{~h}$, the product was isolated by flash chromatography as a colorless oil (50 mg, 76\%). ${ }^{1}$ H NMR (500 MHz, Chloroform-d) $\delta 7.18(\mathrm{~d}, J=8.4 \mathrm{~Hz}, 2 \mathrm{H}), 7.13(\mathrm{~d}, J=8.4 \mathrm{~Hz}, 2 \mathrm{H}), 5.05-4.63(\mathrm{~m}, 1 \mathrm{H}), 3.70-3.41$ (m, 2H), $2.31(\mathrm{~m}, 1 \mathrm{H}), 1.85(\mathrm{~m}, 3 \mathrm{H}), 1.45$ (s, 3H), 1.17 (s, 6H). ${ }^{\mathbf{1 3}} \mathbf{C}$ NMR (125 MHz, Chloroform- $d$ ) $\delta 154.5,147.8,144.0,126.9 \& 126.7,121.5 \& 120.8,120.5(\mathrm{q}, J=25.7$ $\mathrm{Hz}), 79.5,60.8,47.3 \& 47.1,36.0 \& 34.5,28.5 \& 28.1,23.4 \& 23.3 .{ }^{19}$ F NMR (470 MHz, Chloroform- $\boldsymbol{d}$ ) $\delta-57.83,-58.00$. HRMS (EI-QTOF): calcd for $\mathrm{C}_{16} \mathrm{H}_{20} \mathrm{~F}_{3} \mathrm{NO}_{3} 331.1395$, found 331.1385 .

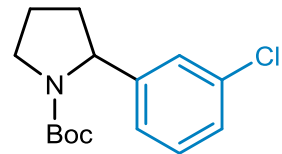

34

tert-butyl 2-(3-chlorophenyl)pyrrolidine-1-carboxylate (34): According to the general procedure, tert-butyl 2-(hydroxymethyl)pyrrolidine-1-carboxylate $(80.5 \mathrm{mg}$, $0.4 \mathrm{mmol}, 2.0$ equiv.), 1-bromo-3-chlorobenzene (38.0 mg, $0.2 \mathrm{mmol}, 1.0$ equiv.), $\mathrm{Ni}(\mathrm{dMebpy})\left(\mathrm{H}_{2} \mathrm{O}\right)_{4} \mathrm{Cl}_{2}(0.8 \mathrm{mg}, 0.002 \mathrm{mmol}, 0.01$ equiv. $), \mathrm{DPA}(0.7 \mathrm{mg}, 0.002 \mathrm{mmol}$, 0.01 equiv.), $\mathbf{L} 4$ (20.0 mg, 0.07 mmol, 0.35 equiv.), $\mathrm{CeCl}_{3}$ (4.9 mg, $0.02 \mathrm{mmol}, 0.1$ equiv.), $\mathrm{Na}_{3} \mathrm{PO}_{4}$ (98.4 mg, $0.6 \mathrm{mmol}, 3$ equiv.), $\mathrm{CH}_{3} \mathrm{CN}(2 \mathrm{~mL})$ and DMSO (0.25 mL) were used. After $36 \mathrm{~h}$, the product was isolated by flash chromatography as a colorless 
oil (46 mg, 82\%). ${ }^{\mathbf{1} H}$ NMR (500 MHz, Chloroform-d) $\delta 7.23(\mathrm{t}, J=7.6 \mathrm{~Hz}, 1 \mathrm{H}), 7.17$ $(\mathrm{d}, J=20.8 \mathrm{~Hz}, 2 \mathrm{H}), 7.05(\mathrm{~d}, J=7.4 \mathrm{~Hz}, 1 \mathrm{H}), 4.99-4.65(\mathrm{~m}, 1 \mathrm{H}), 3.68-3.44(\mathrm{~m}, 2 \mathrm{H})$, $2.32(\mathrm{~m}, 1 \mathrm{H}), 1.86(\mathrm{~m}, 3 \mathrm{H}), 1.46(\mathrm{~s}, 3 \mathrm{H}), 1.20$ (s, 6H). ${ }^{13} \mathrm{C}$ NMR (125 MHz, Chloroform-d) $\delta 154.5,147.3,134.1,129.5,126.6,125.8,123.7,79.5,60.9 \& 60.4$, 47.3\&47.1, 35.9\&34.7, 28.5\&28.1, 23.5\&23.2. HRMS (EI-QTOF): calcd for $\mathrm{C}_{15} \mathrm{H}_{20} \mathrm{ClNO}_{2} 281.1183$, found 281.1179 .

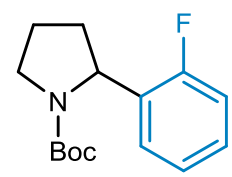

35

tert-butyl 2-(2-fluorophenyl)pyrrolidine-1-carboxylate (35): According to the general procedure, tert-butyl 2-(hydroxymethyl)pyrrolidine-1-carboxylate $(80.5 \mathrm{mg}$, $0.4 \mathrm{mmol}, 2.0$ equiv.), 1-bromo-2-fluorobenzene (35 mg, $0.2 \mathrm{mmol}, 1.0$ equiv.), $\mathrm{Ni}(\mathrm{dMebpy})\left(\mathrm{H}_{2} \mathrm{O}\right)_{4} \mathrm{Cl}_{2}(0.8 \mathrm{mg}, 0.002 \mathrm{mmol}, 0.01$ equiv.), DPA (0.7 mg, $0.002 \mathrm{mmol}$, 0.01 equiv.), $\mathbf{L} 4$ (20.0 mg, $0.07 \mathrm{mmol}, 0.35$ equiv.), $\mathrm{CeCl}_{3}$ (4.9 mg, $0.02 \mathrm{mmol}, 0.1$ equiv.), $\mathrm{Na}_{3} \mathrm{PO}_{4}$ (98.4 mg, $0.6 \mathrm{mmol}, 3$ equiv.), $\mathrm{CH}_{3} \mathrm{CN}(2 \mathrm{~mL})$ and DMSO (0.25 mL) were used. After $36 \mathrm{~h}$, the product was isolated by flash chromatography as a colorless oil (30 mg, 57\%). ${ }^{1}$ H NMR (500 MHz, Chloroform-d) $\delta 7.26-7.05$ (m, 3H), $7.06-$ $6.89(\mathrm{~m}, 1 \mathrm{H}), 5.38-4.94(\mathrm{~m}, 1 \mathrm{H}), 3.64(\mathrm{t}, J=6.8 \mathrm{~Hz}, 2 \mathrm{H}), 2.46-2.22(\mathrm{~m}, 1 \mathrm{H}), 1.88$ (m, 3H), 1.48 (s, 3H), $1.22(\mathrm{~s}, 6 \mathrm{H}) .{ }^{13} \mathbf{C}$ NMR (125 MHz, Chloroform-d) $\delta 159.8$ (d, $J$ $=242.5 \mathrm{~Hz}), 154.3,131.8,128.0(\mathrm{~d}, J=6.3 \mathrm{~Hz}), 126.9 \& 126.5,123.7(\mathrm{~d}, J=3.8 \mathrm{~Hz})$, $115.1(\mathrm{~d}, J=23 \mathrm{~Hz}), 79.3,55.2,46.8,34.4 \& 33.3,28.5 \& 28.1,23.3 .{ }^{19}$ F NMR (470 MHz, Chloroform-d) $\delta-118.63,-119.94$. HRMS (ESI+): calcd for $\mathrm{C}_{15} \mathrm{H}_{21} \mathrm{FNO}_{2}$ $(\mathrm{M}+\mathrm{H})$ 266.1551, found 266.1551 


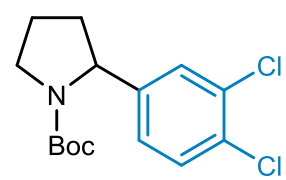

36

tert-butyl 2-(3,4-dichlorophenyl)pyrrolidine-1-carboxylate (36): According to the general procedure, tert-butyl 2-(hydroxymethyl)pyrrolidine-1-carboxylate (80.5 mg, $0.4 \mathrm{mmol}, 2.0$ equiv.), 4-bromo-1,2-dichlorobenzene (45.2 mg, $0.2 \mathrm{mmol}, 1.0$ equiv.), $\mathrm{Ni}(\mathrm{dMebpy})\left(\mathrm{H}_{2} \mathrm{O}\right)_{4} \mathrm{Cl}_{2}(0.8 \mathrm{mg}, 0.002 \mathrm{mmol}, 0.01$ equiv. $)$, DPA (0.7 mg, $0.002 \mathrm{mmol}$, 0.01 equiv.), $\mathbf{L} 4$ (20.0 mg, $0.07 \mathrm{mmol}, 0.35$ equiv.), $\mathrm{CeCl}_{3}$ (4.9 mg, $0.02 \mathrm{mmol}, 0.1$ equiv.), $\mathrm{Na}_{3} \mathrm{PO}_{4}$ (98.4 mg, $0.6 \mathrm{mmol}, 3$ equiv.), $\mathrm{CH}_{3} \mathrm{CN}$ (2 mL) and DMSO (0.25 mL) were used. After $36 \mathrm{~h}$, the product was isolated by flash chromatography as a colorless oil (38 mg, 60\%). ${ }^{\mathbf{1} H}$ NMR (500 MHz, Chloroform- $d$ ) $\delta 7.37$ (d, $\left.J=8.2 \mathrm{~Hz}, 1 \mathrm{H}\right), 7.26$ (s, 1H), $7.01(\mathrm{~d}, J=8.3 \mathrm{~Hz}, 1 \mathrm{H}), 4.95-4.64(\mathrm{~m}, 1 \mathrm{H}), 3.68-3.42(\mathrm{~m}, 2 \mathrm{H}), 2.31(\mathrm{~m}$, 1H), $1.83(\mathrm{~m}, 3 \mathrm{H}), 1.46(\mathrm{~s}, 3 \mathrm{H}), 1.23(\mathrm{~s}, 6 \mathrm{H}) .{ }^{13} \mathrm{C}$ NMR (125 MHz, Chloroform-d) $\delta$ $154.3,145.5 \& 144.6,132.4 \& 132.2,130.3,130.2 \& 130.1,127.6 \& 127.4,124.9,79.7$, 60.4\&59.9, 47.4\&47.0, 35.8\&34.7, 28.4\&28.2, 23.5\&23.1. HRMS (EI-QTOF): calcd for $\mathrm{C}_{15} \mathrm{H}_{19} \mathrm{Cl}_{2} \mathrm{NO}_{2} 315.0793$, found 315.0802.

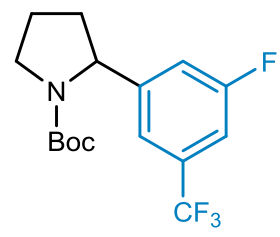

37

tert-butyl 2-(3-fluoro-5-(trifluoromethyl)phenyl)pyrrolidine-1-carboxylate (37): According to the general procedure, tert-butyl 2-(hydroxymethyl)pyrrolidine-1carboxylate (80.5 mg, $0.4 \mathrm{mmol}, \quad 2.0$ equiv.), 1-bromo-3-fluoro-5(trifluoromethyl)benzene (48.6 mg, $0.2 \mathrm{mmol}, 1.0$ equiv.), $\mathrm{Ni}(\mathrm{dMebpy})\left(\mathrm{H}_{2} \mathrm{O}\right)_{4} \mathrm{Cl}_{2}(0.8$ mg, 0.002 mmol, 0.01 equiv.), DPA (0.7 mg, $0.002 \mathrm{mmol}, 0.01$ equiv.), L4 (20.0 mg, 0.07 mmol, 0.35 equiv.), $\mathrm{CeCl}_{3}$ (4.9 mg, $0.02 \mathrm{mmol}, 0.1$ equiv.), $\mathrm{Na}_{3} \mathrm{PO}_{4}$ (98.4 mg, 0.6 mmol, 3 equiv.), $\mathrm{CH}_{3} \mathrm{CN}(2 \mathrm{~mL})$ and DMSO $(0.25 \mathrm{~mL})$ were used. After $36 \mathrm{~h}$, the 
product was isolated by flash chromatography as a colorless oil (43 mg, 65\%). ${ }^{1} \mathbf{H}$ NMR (500 MHz, Chloroform-d) $\delta 7.24$ (s, 1H), 7.19 (d, $J=9.0 \mathrm{~Hz}, 1 \mathrm{H}), 7.08$ (d, $J=9.4 \mathrm{~Hz}$, $1 \mathrm{H}), 5.03-4.71(\mathrm{~m}, 1 \mathrm{H}), 3.69-3.50(\mathrm{~m}, 2 \mathrm{H}), 2.37(\mathrm{~m}, 1 \mathrm{H}), 1.86(\mathrm{~m}, 3 \mathrm{H}), 1.46(\mathrm{~s}, 3 \mathrm{H})$, 1.20 (s, 6H). ${ }^{13}$ C NMR (125 MHz, Chloroform-d) $\delta 162.5(\mathrm{~d}, J=248.2 \mathrm{~Hz})$, 154.5\&154.3, 149.4\&148.4, 132.4 (q, $J=32.8 \mathrm{~Hz}), 123.3(\mathrm{q}, J=273.4 \mathrm{~Hz})$, 118.2\&118.0, $116.0(\mathrm{~d}, J=22.7 \mathrm{~Hz}), 111.0(\mathrm{~d}, J=23.9 \mathrm{~Hz}), 79.9,60.8 \& 60.2$, 47.4\&47.1, 35.9\&34.7, 28.4\&28.1, 23.5\&23.3. ${ }^{19}$ F NMR (470 MHz, Chloroform-d) $\delta$-62.76, -111.12. HRMS (EI-QTOF): calcd for $\mathrm{C}_{16} \mathrm{H}_{19} \mathrm{~F}_{4} \mathrm{NO}_{2}$ 333.1352, found 333.1359 .

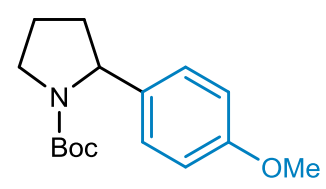

38

tert-butyl 2-(4-methoxyphenyl)pyrrolidine-1-carboxylate (38): According to the general procedure, tert-butyl 2-(hydroxymethyl)pyrrolidine-1-carboxylate $(80.5 \mathrm{mg}$, $0.4 \mathrm{mmol}, 2.0$ equiv.), 1-bromo-4-methoxybenzene (37.2 $\mathrm{mg}, 0.2 \mathrm{mmol}, 1.0$ equiv.), $\mathrm{Ni}(\mathrm{dMebpy})\left(\mathrm{H}_{2} \mathrm{O}\right)_{4} \mathrm{Cl}_{2}(0.8 \mathrm{mg}, 0.002 \mathrm{mmol}, 0.01$ equiv.), DPA (0.7 mg, $0.002 \mathrm{mmol}$, 0.01 equiv.), $\mathbf{L} 4$ (20.0 mg, $0.07 \mathrm{mmol}, 0.35$ equiv.), $\mathrm{CeCl}_{3}$ (4.9 mg, $0.02 \mathrm{mmol}, 0.1$ equiv.), $\mathrm{Na}_{3} \mathrm{PO}_{4}$ (98.4 mg, $0.6 \mathrm{mmol}, 3$ equiv.), $\mathrm{CH}_{3} \mathrm{CN}$ (2 mL) and DMSO (0.25 mL) were used. After $36 \mathrm{~h}$, the product was isolated by flash chromatography as a colorless oil (37 mg, 67\%). ${ }^{\mathbf{1}} \mathbf{H}$ NMR (500 MHz, Chloroform-d) $\delta 7.08(\mathrm{~d}, \mathrm{~J}=8.0 \mathrm{~Hz}, 2 \mathrm{H}), 6.88$ - $6.77(\mathrm{~m}, 2 \mathrm{H}), 4.82(\mathrm{~m}, 1 \mathrm{H}), 3.80(\mathrm{~s}, 3 \mathrm{H}), 3.55(\mathrm{~m}, 2 \mathrm{H}), 2.26(\mathrm{~m}, 1 \mathrm{H}), 1.84(\mathrm{~m}, 3 \mathrm{H})$, 1.45 (s, 3H), 1.20 (s, 6H). ${ }^{13}$ C NMR (125 MHz, Chloroform-d) $\delta$ 158.3, 154.6, 126.6, 125.5, 113.5, 79.1, 60.7, 55.3, 47.1, 36.0, 28.3, 23.2. HRMS (ESI+): calcd for $\mathrm{C}_{16} \mathrm{H}_{23} \mathrm{NO}_{3} \mathrm{Na}(\mathrm{M}+\mathrm{Na}) 300.1570$, found 300.1573 . 


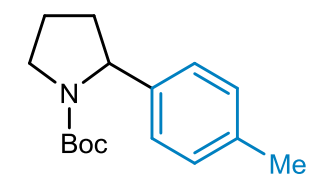

39

tert-butyl 2-(p-tolyl)pyrrolidine-1-carboxylate (39): According to the general procedure, tert-butyl 2-(hydroxymethyl)pyrrolidine-1-carboxylate $(80.5 \mathrm{mg}, 0.4 \mathrm{mmol}$, 2.0 equiv.), 1-bromo-4-methylbenzene (34.2 mg, $0.2 \mathrm{mmol}, 1.0$ equiv.), $\mathrm{Ni}(\mathrm{dMebpy})\left(\mathrm{H}_{2} \mathrm{O}\right){ }_{4} \mathrm{Cl}_{2}(0.8 \mathrm{mg}, 0.002 \mathrm{mmol}, 0.01$ equiv. $)$, DPA (0.7 mg, $0.002 \mathrm{mmol}$, 0.01 equiv.), $\mathbf{L} 4$ (20.0 mg, $0.07 \mathrm{mmol}, 0.35$ equiv.), $\mathrm{CeCl}_{3}$ (4.9 mg, $0.02 \mathrm{mmol}, 0.1$ equiv.), $\mathrm{Na}_{3} \mathrm{PO}_{4}$ (98.4 mg, $0.6 \mathrm{mmol}, 3$ equiv.), $\mathrm{CH}_{3} \mathrm{CN}(2 \mathrm{~mL})$ and DMSO (0.25 mL) were used. After $36 \mathrm{~h}$, the product was isolated by flash chromatography as a colorless oil (35 mg, 67\%). ${ }^{1} \mathbf{H}$ NMR (500 MHz, Chloroform-d) $\delta 7.10$ (d, J=7.6 Hz, 2H), 7.04 $(\mathrm{d}, J=8.1 \mathrm{~Hz}, 2 \mathrm{H}), 5.00-4.67(\mathrm{~m}, 1 \mathrm{H}), 3.69-3.42(\mathrm{~m}, 2 \mathrm{H}), 2.32(\mathrm{~m}, 4 \mathrm{H}), 1.96-1.74$ (m, 3H), 1.45 (s, 3H), 1.19 (s, 6H). ${ }^{13}$ C NMR (125 MHz, Chloroform-d) $\delta$ 154.7, $142.0,135.9,129.0 \& 128.7,125.4,79.2,61.0 \& 60.6,47.0,36.0 \& 34.8,28.5 \& 28.2,23.1$, 21.0. HRMS (EI-QTOF): calcd for $\mathrm{C}_{16} \mathrm{H}_{23} \mathrm{NO}_{2}$ 261.1729, found 261.1725.

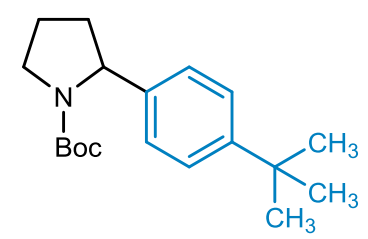

40

tert-butyl 2-(4-(tert-butyl)phenyl)pyrrolidine-1-carboxylate (40): According to the general procedure, tert-butyl 2-(hydroxymethyl)pyrrolidine-1-carboxylate $(80.5 \mathrm{mg}$, $0.4 \mathrm{mmol}, 2.0$ equiv.), 1-bromo-4-(tert-butyl)benzene (42.4 mg, $0.2 \mathrm{mmol}, 1.0$ equiv.), $\mathrm{Ni}(\mathrm{dMebpy})\left(\mathrm{H}_{2} \mathrm{O}\right)_{4} \mathrm{Cl}_{2}(0.8 \mathrm{mg}, 0.002 \mathrm{mmol}, 0.01$ equiv.), DPA (0.7 mg, $0.002 \mathrm{mmol}$, 0.01 equiv.), $\mathbf{L} 4$ (20.0 mg, $0.07 \mathrm{mmol}, 0.35$ equiv.), $\mathrm{CeCl}_{3}$ (4.9 mg, $0.02 \mathrm{mmol}, 0.1$ equiv.), $\mathrm{Na}_{3} \mathrm{PO}_{4}$ (98.4 mg, $0.6 \mathrm{mmol}, 3$ equiv.), $\mathrm{CH}_{3} \mathrm{CN}(2 \mathrm{~mL})$ and DMSO (0.25 mL) were used. After $36 \mathrm{~h}$, the product was isolated by flash chromatography as a colorless oil (48 mg, 79\%). ${ }^{1} \mathbf{H}$ NMR (500 MHz, Chloroform-d) $\delta 7.30$ (d, $J=8.0$ Hz, 2H), 7.08 
$(\mathrm{d}, J=8.0 \mathrm{~Hz}, 2 \mathrm{H}), 5.05-4.61(\mathrm{~m}, 1 \mathrm{H}), 3.67-3.44(\mathrm{~m}, 2 \mathrm{H}), 2.39-2.17(\mathrm{~m}, 1 \mathrm{H}), 1.95$ $-1.78(\mathrm{~m}, 3 \mathrm{H}), 1.46(\mathrm{~s}, 3 \mathrm{H}), 1.30$ (s, 9H), 1.16 (s, 6H). ${ }^{13} \mathrm{C}$ NMR (125 MHz, Chloroform- $d) \quad \delta \quad 154.7,149.3,141.9 \& 140.8, \quad 125.3,124.8,79.1,61.0 \& 60.2$, 47.2\&47.0, 35.9, 34.6\&34.4, 31.4, 28.5\&28.1, 23.3. HRMS (ESI+): calcd for $\mathrm{C}_{19} \mathrm{H}_{29} \mathrm{NO}_{2} \mathrm{Na}(\mathrm{M}+\mathrm{Na}) 326.2091$, found 326.2093.

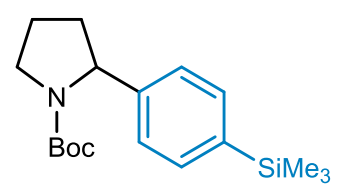

41

tert-butyl 2-(4-(trimethylsilyl)phenyl)pyrrolidine-1-carboxylate (41): According to the general procedure, tert-butyl 2-(hydroxymethyl)pyrrolidine-1-carboxylate $(80.5 \mathrm{mg}$, 0.4 mmol, 2.0 equiv.), (4-bromophenyl)trimethylsilane (45.8 mg, $0.2 \mathrm{mmol}, 1.0$ equiv.), $\mathrm{Ni}(\mathrm{dMebpy})\left(\mathrm{H}_{2} \mathrm{O}\right)_{4} \mathrm{Cl}_{2}(0.8 \mathrm{mg}, 0.002 \mathrm{mmol}, 0.01$ equiv.), DPA (0.7 mg, $0.002 \mathrm{mmol}$, 0.01 equiv.), $\mathbf{L} 4$ (20.0 mg, 0.07 mmol, 0.35 equiv.), $\mathrm{CeCl}_{3}$ (4.9 mg, $0.02 \mathrm{mmol}, 0.1$ equiv.), $\mathrm{Na}_{3} \mathrm{PO}_{4}$ (98.4 mg, $0.6 \mathrm{mmol}, 3$ equiv.), $\mathrm{CH}_{3} \mathrm{CN}(2 \mathrm{~mL})$ and DMSO (0.25 mL) were used. After $36 \mathrm{~h}$, the product was isolated by flash chromatography as a colorless oil (47 mg, 74\%). ${ }^{1}$ H NMR (500 MHz, Chloroform-d) $\delta 7.43$ (d, J=7.4 Hz, 2H), 7.14 $(\mathrm{d}, J=7.4 \mathrm{~Hz}, 2 \mathrm{H}), 5.04-4.65(\mathrm{~m}, 1 \mathrm{H}), 3.67-3.44(\mathrm{~m}, 2 \mathrm{H}), 2.39-2.17(\mathrm{~m}, 1 \mathrm{H}), 1.85$ (m, 3H), 1.45 (s, 3H), 1.17 (s, 6H), 0.24 (s, 9H). ${ }^{13}$ C NMR (125 MHz, Chloroform-d) $\delta 154.6,145.5 \& 144.5,138.2,133.4 \& 133.1,125.0 \& 124.7,79.2,61.3 \& 60.6,47.3 \& 47.0$, 35.9\&34.7, 28.5\&28.1, 23.4\&23.2, -1.1. HRMS (ESI+): calcd for $\mathrm{C}_{18} \mathrm{H}_{29} \mathrm{NO}_{2} \mathrm{SiNa}$ (M+Na) 342.1860, found 342.1864.

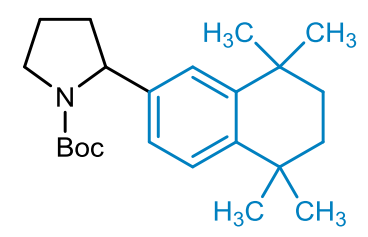

42 
tert-butyl 2-(5,5,8,8-tetramethyl-5,6,7,8-tetrahydronaphthalen-2-yl)pyrrolidine-1carboxylate (42): According to the general procedure, tert-butyl 2(hydroxymethyl)pyrrolidine-1-carboxylate ( $80.5 \mathrm{mg}, 0.4 \mathrm{mmol}, 2.0$ equiv.), 6-bromo1,1,4,4-tetramethyl-1,2,3,4-tetrahydronaphthalene (53.4 mg, $0.2 \mathrm{mmol}, 1.0$ equiv.), $\mathrm{Ni}(\mathrm{dMebpy})\left(\mathrm{H}_{2} \mathrm{O}\right)_{4} \mathrm{Cl}_{2}(0.8 \mathrm{mg}, 0.002 \mathrm{mmol}, 0.01$ equiv.), DPA (0.7 mg, $0.002 \mathrm{mmol}$, 0.01 equiv.), $\mathbf{L 4}$ (20.0 mg, 0.07 mmol, 0.35 equiv.), $\mathrm{CeCl}_{3}$ (4.9 mg, $0.02 \mathrm{mmol}, 0.1$ equiv.), $\mathrm{Na}_{3} \mathrm{PO}_{4}$ (98.4 mg, $0.6 \mathrm{mmol}, 3$ equiv.), $\mathrm{CH}_{3} \mathrm{CN}(2 \mathrm{~mL})$ and DMSO (0.25 mL) were used. After $36 \mathrm{~h}$, the product was isolated by flash chromatography as a colorless oil (48 mg, 67\%). ${ }^{1} \mathbf{H}$ NMR (500 MHz, Chloroform-d) $\delta 7.20(\mathrm{~d}, J=8.1 \mathrm{~Hz}, 1 \mathrm{H}), 7.04$ $(\mathrm{s}, 1 \mathrm{H}), 6.90(\mathrm{~d}, J=8.0 \mathrm{~Hz}, 1 \mathrm{H}), 4.81(\mathrm{~m}, 1 \mathrm{H}), 3.68-3.41(\mathrm{~m}, 2 \mathrm{H}), 2.38-2.17(\mathrm{~m}$, $1 \mathrm{H}), 1.88(\mathrm{~m}, 3 \mathrm{H}), 1.66(\mathrm{~s}, 4 \mathrm{H}), 1.47(\mathrm{~s}, 3 \mathrm{H}), 1.28-1.22(\mathrm{~m}, 12 \mathrm{H}), 1.15(\mathrm{~s}, 6 \mathrm{H}) .{ }^{13} \mathrm{C}$ NMR (125 MHz, Chloroform-d) $\delta$ 154.7, 144.3, 142.9, 142.0, 126.2, 123.4, 122.9, 79.1, 61.5, 47.1, 36.0, 35.2\&35.1, 34.2, 34.0, 32.0, 31.90, 28.6\&28.2, 23.3. HRMS (ESI+): calcd for $\mathrm{C}_{23} \mathrm{H}_{35} \mathrm{NO}_{2} \mathrm{Na}(\mathrm{M}+\mathrm{Na}) 380.2560$, found 380.2565.

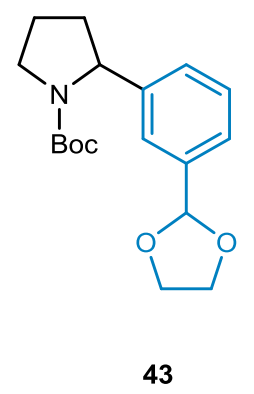

tert-butyl 2-(3-(1,3-dioxolan-2-yl)phenyl)pyrrolidine-1-carboxylate (43): According to the general procedure, tert-butyl 2-(hydroxymethyl)pyrrolidine-1carboxylate ( $80.5 \mathrm{mg}, 0.4 \mathrm{mmol}, 2.0$ equiv.), 2-(3-bromophenyl)-1,3-dioxolane (45.8 mg, $0.2 \mathrm{mmol}, 1.0$ equiv.), $\mathrm{Ni}(\mathrm{dMebpy})\left(\mathrm{H}_{2} \mathrm{O}\right)_{4} \mathrm{Cl}_{2}(0.8 \mathrm{mg}, 0.002 \mathrm{mmol}, 0.01$ equiv.), DPA (0.7 mg, 0.002 mmol, 0.01 equiv.), $\mathbf{L 4}$ (20.0 mg, 0.07 mmol, 0.35 equiv.), $\mathrm{CeCl}_{3}$ (4.9 mg, 0.02 mmol, 0.1 equiv.), $\mathrm{Na}_{3} \mathrm{PO}_{4}$ (98.4 mg, 0.6 mmol, 3 equiv.), $\mathrm{CH}_{3} \mathrm{CN}$ (2 mL) and DMSO $(0.25 \mathrm{~mL})$ were used. After $36 \mathrm{~h}$, the product was isolated by flash chromatography as a colorless oil (51 mg, 80\%). ${ }^{\mathbf{1}}$ H NMR (500 MHz, Chloroform- ) 
$\delta 7.30-7.16(\mathrm{~m}, 3 \mathrm{H}), 7.14-7.04(\mathrm{~m}, 1 \mathrm{H}), 5.71(\mathrm{~s}, 1 \mathrm{H}), 4.80(\mathrm{~m}, 1 \mathrm{H}), 4.23-3.74(\mathrm{~m}$, 4H), $3.55(\mathrm{~s}, 2 \mathrm{H}), 2.24(\mathrm{~s}, 1 \mathrm{H}), 1.87-1.72(\mathrm{~m}, 3 \mathrm{H}), 1.38(\mathrm{~s}, 3 \mathrm{H}), 1.11(\mathrm{~s}, 6 \mathrm{H}) .{ }^{13} \mathrm{C}$ NMR (126 MHz, Chloroform-d) $\delta$ 154.6, 137.8, 128.2, 126.4, 124.6, 123.7, 103.8, 79.3, 65.2, 63.7, 61.3, 47.1, 36.0, 28.1, 23.3. HRMS (ESI+): calcd for $\mathrm{C}_{18} \mathrm{H}_{25} \mathrm{NO}_{4} \mathrm{Na}$ $(\mathrm{M}+\mathrm{Na}) 342.1681$, found 342.1685.

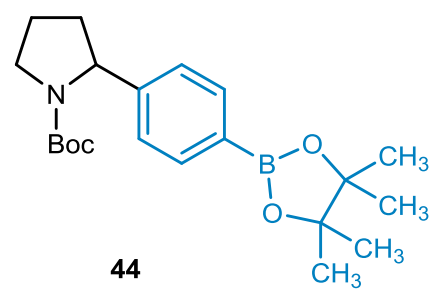

tert-butyl 2-(4-(4,4,5,5-tetramethyl-1,3,2-dioxaborolan-2-yl)phenyl)pyrrolidine-1carboxylate (44): According to the general procedure, tert-butyl 2(hydroxymethyl)pyrrolidine-1-carboxylate $(80.5 \mathrm{mg}, 0.4 \mathrm{mmol}, 2.0$ equiv.), 2-(4bromophenyl)-4,4,5,5-tetramethyl-1,3,2-dioxaborolane (56.6 mg, $0.2 \mathrm{mmol}, 1.0$ equiv.), $\mathrm{Ni}(\mathrm{dMebpy})\left(\mathrm{H}_{2} \mathrm{O}\right)_{4} \mathrm{Cl}_{2}(0.8 \mathrm{mg}, 0.002 \mathrm{mmol}, 0.01$ equiv.), DPA (0.7 mg, 0.002 mmol, 0.01 equiv.), $\mathbf{L 4}$ (20.0 mg, 0.07 mmol, 0.35 equiv.), $\mathrm{CeCl}_{3}$ (4.9 mg, $0.02 \mathrm{mmol}$, 0.1 equiv.), $\mathrm{Na}_{3} \mathrm{PO}_{4}$ (98.4 mg, $0.6 \mathrm{mmol}, 3$ equiv.), $\mathrm{CH}_{3} \mathrm{CN}$ (2 mL) and DMSO (0.25 $\mathrm{mL}$ ) were used. After $36 \mathrm{~h}$, the product was isolated by flash chromatography as a colorless oil (57 mg, 76\%). ${ }^{1}$ H NMR (500 MHz, Chloroform-d) $\delta 7.74$ (d, J=7.4 Hz, 2H), $7.17(\mathrm{~d}, J=7.5 \mathrm{~Hz}, 2 \mathrm{H}), 4.87(\mathrm{~m}, 1 \mathrm{H}), 3.57(\mathrm{~m}, 2 \mathrm{H}), 2.31(\mathrm{~s}, 1 \mathrm{H}), 1.96-1.74(\mathrm{~m}$, 3H), 1.45 (s, 3H), 1.34 (s, 12H), 1.19 (s, 6H). ${ }^{13}$ C NMR (125 MHz, Chloroform-d) $\delta$ $148.3,134.7,124.8,83.7,79.3,61.4,47.1,35.9,28.5 \& 28.2,24.9 \& 24.8,23.1$. HRMS (ESI+): calcd for $\mathrm{C}_{21} \mathrm{H}_{32} \mathrm{BNO}_{4} \mathrm{Na}(\mathrm{M}+\mathrm{Na}) 396.2317$, found 396.2323.

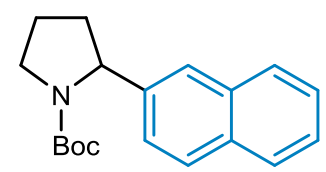


tert-butyl 2-(naphthalen-2-yl)pyrrolidine-1-carboxylate (45): According to the general procedure, tert-butyl 2-(hydroxymethyl)pyrrolidine-1-carboxylate $(80.5 \mathrm{mg}$, $0.4 \mathrm{mmol}, 2.0$ equiv.), 2-bromonaphthalene (41.4 mg, $0.2 \mathrm{mmol}, 1.0$ equiv.), $\mathrm{Ni}(\mathrm{dMebpy})\left(\mathrm{H}_{2} \mathrm{O}\right)_{4} \mathrm{Cl}_{2}(0.8 \mathrm{mg}, 0.002 \mathrm{mmol}, 0.01$ equiv.), DPA (0.7 mg, $0.002 \mathrm{mmol}$, 0.01 equiv.), $\mathbf{L} 4$ (20.0 mg, $0.07 \mathrm{mmol}, 0.35$ equiv.), $\mathrm{CeCl}_{3}$ (4.9 mg, $0.02 \mathrm{mmol}, 0.1$ equiv.), $\mathrm{Na}_{3} \mathrm{PO}_{4}$ (98.4 mg, $0.6 \mathrm{mmol}, 3$ equiv.), $\mathrm{CH}_{3} \mathrm{CN}(2 \mathrm{~mL})$ and DMSO (0.25 mL) were used. After $36 \mathrm{~h}$, the product was isolated by flash chromatography as a white solid (27 mg, 46\%). ${ }^{1}$ H NMR (500 MHz, Chloroform-d) $\delta 7.86-7.75$ (m, 3H), 7.58 (s, 1H), $7.45(\mathrm{p}, J=6.8 \mathrm{~Hz}, 2 \mathrm{H}), 7.31(\mathrm{~d}, J=8.5 \mathrm{~Hz}, 1 \mathrm{H}), 5.20-4.86(\mathrm{~m}, 1 \mathrm{H}), 3.79-$ $3.52(\mathrm{~m}, 2 \mathrm{H}), 2.38(\mathrm{~m}, 1 \mathrm{H}), 2.01-1.85(\mathrm{~m}, 3 \mathrm{H}), 1.47(\mathrm{~s}, 3 \mathrm{H}), 1.14(\mathrm{~s}, 6 \mathrm{H}) .{ }^{13} \mathrm{C}$ NMR (125 MHz, Chloroform-d) $\delta$ 154.7, 142.4, 133.2, 132.4, 128.2, 128.0\&127.6, 126.0, $125.3,124.1,123.8,79.3,61.4 \& 60.8,47.5 \& 47.1,35.8 \& 34.7,28.5 \& 28.1,23.5 \& 23.1$. HRMS (ESI+): calcd for $\mathrm{C}_{19} \mathrm{H}_{23} \mathrm{NO}_{2} \mathrm{Na}(\mathrm{M}+\mathrm{Na}) 320.1621$, found 320.1624 .

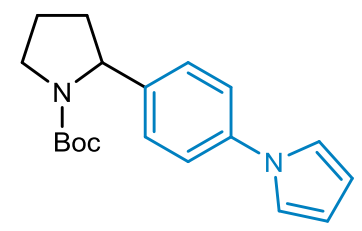

46

tert-butyl 2-(4-(1H-pyrrol-1-yl)phenyl)pyrrolidine-1-carboxylate (46): According to the general procedure, tert-butyl 2-(hydroxymethyl)pyrrolidine-1-carboxylate $(80.5$ mg, 0.4 mmol, 2.0 equiv.), 1-(4-bromophenyl)-1H-pyrrole (44.4 mg, $0.2 \mathrm{mmol}, 1.0$ equiv.), $\mathrm{Ni}(\mathrm{dMebpy})\left(\mathrm{H}_{2} \mathrm{O}\right)_{4} \mathrm{Cl}_{2}$ (0.8 mg, $0.002 \mathrm{mmol}, 0.01$ equiv.), DPA (0.7 mg, 0.002 mmol, 0.01 equiv.), $\mathbf{L 4}$ (20.0 mg, 0.07 mmol, 0.35 equiv.), $\mathrm{CeCl}_{3}$ (4.9 mg, $0.02 \mathrm{mmol}$, 0.1 equiv.), $\mathrm{Na}_{3} \mathrm{PO}_{4}$ (98.4 mg, $0.6 \mathrm{mmol}, 3$ equiv.), $\mathrm{CH}_{3} \mathrm{CN}$ (2 mL) and DMSO (0.25 $\mathrm{mL}$ ) were used. After $36 \mathrm{~h}$, the product was isolated by flash chromatography as a yellow oil (47 mg, 75\%). ${ }^{1}$ H NMR (500 MHz, Chloroform- $d$ ) $\delta 7.33(\mathrm{~d}, J=8.1 \mathrm{~Hz}$, 2H), $7.23(\mathrm{~d}, J=8.2 \mathrm{~Hz}, 2 \mathrm{H}), 7.08(\mathrm{~m}, 2 \mathrm{H}), 6.34(\mathrm{~m}, 2 \mathrm{H}), 5.05-4.72(\mathrm{~m}, 1 \mathrm{H}), 3.71-$ $3.48(\mathrm{~m}, 2 \mathrm{H}), 2.36(\mathrm{~m}, 1 \mathrm{H}), 1.89$ (m, 3H), $1.47(\mathrm{~s}, 3 \mathrm{H}), 1.21(\mathrm{~s}, 6 \mathrm{H}) .{ }^{13} \mathbf{C}$ NMR (125 MHz, Chloroform- $d$ ) $\delta$ 154.5, 142.6, 139.2, 126.6, 120.8\&120.1, 119.2, 110.2\&110.0, 
79.4, 60.8\&60.2, 47.1, 36.0\&34.8, 28.5\&28.2, 23.5\&23.2. HRMS (ESI+): calcd for $\mathrm{C}_{19} \mathrm{H}_{25} \mathrm{~N}_{2} \mathrm{O}_{2}(\mathrm{M}+\mathrm{H})$ 313.1911, found 313.1913.

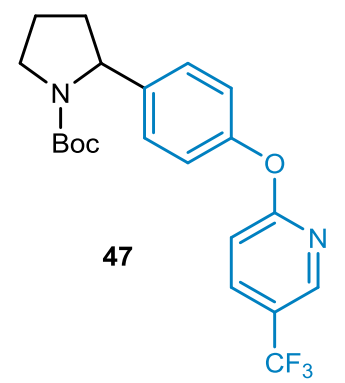

tert-butyl 2-(4-((5-(trifluoromethyl)pyridin-2-yl)oxy)phenyl)pyrrolidine-1carboxylate (47): According to the general procedure, tert-butyl 2(hydroxymethyl)pyrrolidine-1-carboxylate $(80.5 \mathrm{mg}, 0.4 \mathrm{mmol}, 2.0$ equiv.), 2-(4bromophenoxy)-5-(trifluoromethyl)pyridine (63.6 mg, $0.2 \mathrm{mmol}, 1.0$ equiv.), $\mathrm{Ni}(\mathrm{dMebpy})\left(\mathrm{H}_{2} \mathrm{O}\right)_{4} \mathrm{Cl}_{2}(0.8 \mathrm{mg}, 0.002 \mathrm{mmol}, 0.01$ equiv.), DPA (0.7 mg, $0.002 \mathrm{mmol}$, 0.01 equiv.), $\mathbf{L} 4$ (20.0 mg, $0.07 \mathrm{mmol}, 0.35$ equiv.), $\mathrm{CeCl}_{3}$ (4.9 mg, $0.02 \mathrm{mmol}, 0.1$ equiv.), $\mathrm{Na}_{3} \mathrm{PO}_{4}$ (98.4 mg, $0.6 \mathrm{mmol}, 3$ equiv.), $\mathrm{CH}_{3} \mathrm{CN}(2 \mathrm{~mL})$ and DMSO (0.25 mL) were used. After $36 \mathrm{~h}$, the product was isolated by flash chromatography as a colorless oil (62 mg, 76\%). ${ }^{1} \mathbf{H}$ NMR (500 MHz, Chloroform-d) $\delta 8.48(\mathrm{~d}, J=16.1 \mathrm{~Hz}, 1 \mathrm{H})$, $7.89(\mathrm{~d}, J=8.6 \mathrm{~Hz}, 1 \mathrm{H}), 7.23(\mathrm{~d}, J=7.9 \mathrm{~Hz}, 2 \mathrm{H}), 7.08(\mathrm{~d}, J=8.0 \mathrm{~Hz}, 2 \mathrm{H}), 6.98(\mathrm{~d}, J=$ $8.7 \mathrm{~Hz}, 1 \mathrm{H}), 4.89(\mathrm{~m}, 1 \mathrm{H}), 3.74-3.43(\mathrm{~m}, 2 \mathrm{H}), 2.49-2.20(\mathrm{~m}, 1 \mathrm{H}), 2.00-1.81(\mathrm{~m}$, 3H), 1.47 (s, 3H), 1.22 (s, 6H). ${ }^{13}$ C NMR (125 MHz, Chloroform-d) $\delta$ 165.9, 154.6, 151.6, $145.5(\mathrm{q}, J=5.0 \mathrm{~Hz}), 142.3,136.7,127.0,123.7(\mathrm{q}, J=272.2 \mathrm{~Hz}), 121.5(\mathrm{q}, J=$ $34.0 \mathrm{~Hz}), 121.0,111.1,79.4,60.9 \& 60.2,47.3 \& 47.1,35.9 \& 34.7,28.5 \& 28.2,23.5 \& 23.3$. ${ }^{19} \mathrm{~F}$ NMR (470 MHz, Chloroform- $d$ ) $\delta$-61.64. HRMS (ESI+): calcd for $\mathrm{C}_{21} \mathrm{H}_{23} \mathrm{~F}_{3} \mathrm{~N}_{2} \mathrm{O}_{3} \mathrm{Na}(\mathrm{M}+\mathrm{Na})$ 431.1553, found 431.1561.

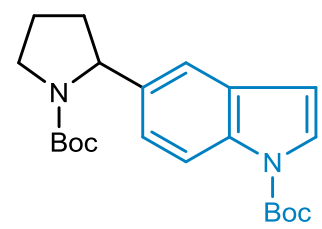


tert-butyl 5-(1-(tert-butoxycarbonyl)pyrrolidin-2-yl)-1H-indole-1-carboxylate (48): According to the general procedure, tert-butyl 2-(hydroxymethyl)pyrrolidine-1carboxylate (80.5 mg, $0.4 \mathrm{mmol}, 2.0$ equiv.), tert-butyl 5-bromo-1H-indole-1carboxylate (59.2 mg, $0.2 \mathrm{mmol}, 1.0$ equiv.), $\mathrm{Ni}(\mathrm{dMebpy})\left(\mathrm{H}_{2} \mathrm{O}\right)_{4} \mathrm{Cl}_{2}(0.8 \mathrm{mg}, 0.002$ mmol, 0.01 equiv.), DPA (0.7 mg, $0.002 \mathrm{mmol}, 0.01$ equiv.), L4 (20.0 mg, $0.07 \mathrm{mmol}$, 0.35 equiv.), $\mathrm{CeCl}_{3}$ (4.9 mg, $0.02 \mathrm{mmol}, 0.1$ equiv.), $\mathrm{Na}_{3} \mathrm{PO}_{4}$ (98.4 mg, $0.6 \mathrm{mmol}, 3$ equiv.), $\mathrm{CH}_{3} \mathrm{CN}(2 \mathrm{~mL})$ and DMSO $(0.25 \mathrm{~mL})$ were used. After $36 \mathrm{~h}$, the product was isolated by flash chromatography as a white solid (42 mg, 54\%). ${ }^{1} \mathbf{H}$ NMR (500 MHz, Chloroform- $d$ ) $\delta 8.04(\mathrm{~d}, J=8.0 \mathrm{~Hz}, 1 \mathrm{H}), 7.57$ (s, 1H), 7.34 (d, $J=8.5 \mathrm{~Hz}, 1 \mathrm{H}), 7.12$ $(\mathrm{d}, J=8.8 \mathrm{~Hz}, 1 \mathrm{H}), 6.51(\mathrm{~d}, J=3.7 \mathrm{~Hz}, 1 \mathrm{H}), 5.11-4.79(\mathrm{~m}, 1 \mathrm{H}), 3.75-3.47(\mathrm{~m}, 2 \mathrm{H})$, $2.40-2.22(\mathrm{~m}, 1 \mathrm{H}), 2.02-1.80(\mathrm{~m}, 3 \mathrm{H}), 1.67(\mathrm{~s}, 9 \mathrm{H}), 1.46(\mathrm{~s}, 3 \mathrm{H}), 1.17(\mathrm{~s}, 6 \mathrm{H}) .{ }^{13} \mathrm{C}$ NMR (125 MHz, Chloroform-d) $\delta$ 154.7, 149.8, 139.6, 130.4, 126.1, 122.0, 117.5, 114.7, 107.2, 83.5, 79.1, 61.3\&60.9, 47.0, 36.2\&35.2, 28.5\&28.2, 23.5\&23.0. HRMS (ESI+): calcd for $\mathrm{C}_{22} \mathrm{H}_{31} \mathrm{~N}_{2} \mathrm{O}_{4}(\mathrm{M}+\mathrm{H})$ 387.2284, found 387.2284.

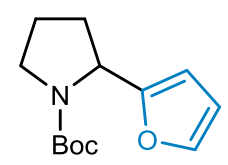

49

tert-butyl 2-(furan-2-yl)pyrrolidine-1-carboxylate (49): According to the general procedure, tert-butyl 2-(hydroxymethyl)pyrrolidine-1-carboxylate $(80.5 \mathrm{mg}, 0.4 \mathrm{mmol}$, 2.0 equiv.), 2-bromofuran (29.4 mg, $0.2 \mathrm{mmol}, 1.0$ equiv.), $\mathrm{Ni}(\mathrm{dMebpy})\left(\mathrm{H}_{2} \mathrm{O}\right)_{4} \mathrm{Cl}_{2}(0.8$ mg, 0.002 mmol, 0.01 equiv.), DPA (0.7 mg, 0.002 mmol, 0.01 equiv.), L4 (20.0 mg, 0.07 mmol, 0.35 equiv.), $\mathrm{CeCl}_{3}$ (4.9 mg, 0.02 mmol, 0.1 equiv.), $\mathrm{Na}_{3} \mathrm{PO}_{4}$ (98.4 mg, 0.6 mmol, 3 equiv.), $\mathrm{CH}_{3} \mathrm{CN}(2 \mathrm{~mL})$ and DMSO $(0.25 \mathrm{~mL})$ were used. After $36 \mathrm{~h}$, the product was isolated by flash chromatography as a colorless oil (27 mg, 57\%). ${ }^{1} \mathbf{H}$ NMR (500 MHz, Chloroform-d) $\delta 7.30(\mathrm{~s}, 1 \mathrm{H}), 6.28(\mathrm{~s}, 1 \mathrm{H}), 6.09$ (d, J=27.1 Hz, 1H), 5.04 - $4.72(\mathrm{~m}, 1 \mathrm{H}), 3.49(\mathrm{~m}, 2 \mathrm{H}), 2.20-1.99(\mathrm{~m}, 3 \mathrm{H}), 1.90(\mathrm{~m}, 1 \mathrm{H}), 1.48-1.42(\mathrm{~m}, 3 \mathrm{H})$, 1.34 (s, 6H). ${ }^{13}$ C NMR (125 MHz, Chloroform-d) $\delta 154.4,141.0,110.0,105.2,79.4$, 
54.8, 46.6\&46.0, 32.2, 28.3, 23.9\&23.3. HRMS (EI-QTOF): calcd for $\mathrm{C}_{13} \mathrm{H}_{19} \mathrm{NO}_{3}$ 237.1365 , found 237.1367 .

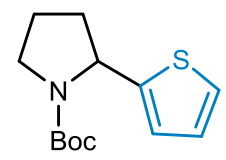

50

tert-butyl 2-(thiophen-2-yl)pyrrolidine-1-carboxylate (50): According to the general procedure, tert-butyl 2-(hydroxymethyl)pyrrolidine-1-carboxylate $(80.5 \mathrm{mg}, 0.4 \mathrm{mmol}$, 2.0 equiv.), 2-bromothiophene (32.4 mg, $0.2 \mathrm{mmol}, 1.0$ equiv.), $\mathrm{Ni}(\mathrm{dMebpy})\left(\mathrm{H}_{2} \mathrm{O}\right)_{4} \mathrm{Cl}_{2}$ (0.8 mg, 0.002 mmol, 0.01 equiv.), DPA (0.7 mg, 0.002 mmol, 0.01 equiv.), L4 (20.0 mg, $0.07 \mathrm{mmol}, 0.35$ equiv.), $\mathrm{CeCl}_{3}$ (4.9 mg, $0.02 \mathrm{mmol}, 0.1$ equiv.), $\mathrm{Na}_{3} \mathrm{PO}_{4}$ (98.4 mg, $0.6 \mathrm{mmol}, 3$ equiv.), $\mathrm{CH}_{3} \mathrm{CN}(2 \mathrm{~mL})$ and $\mathrm{DMSO}(0.25 \mathrm{~mL})$ were used. After $36 \mathrm{~h}$, the product was isolated by flash chromatography as a colorless oil (23 mg, 45\%). ${ }^{1} \mathbf{H}$ NMR (400 MHz, Chloroform-d) $\delta 7.06(\mathrm{~s}, 1 \mathrm{H}), 6.89-6.66(\mathrm{~m}, 2 \mathrm{H}), 5.07(\mathrm{~m}, 1 \mathrm{H}), 3.44(\mathrm{~m}$, 2H), $2.28-2.11(\mathrm{~m}, 1 \mathrm{H}), 2.01-1.79(\mathrm{~m}, 3 \mathrm{H}), 1.30(\mathrm{~s}, 9 \mathrm{H}) .{ }^{\mathbf{1 3}} \mathbf{C}$ NMR (101 MHz, Chloroform-d) $\delta 154.4,148.8,126.4,123.2,123.0,79.5,56.8,46.2,35.5,28.3,23.2$. HRMS (EI-QTOF): calcd for $\mathrm{C}_{13} \mathrm{H}_{19} \mathrm{NO}_{2} \mathrm{~S} 253.1136$, found 253.1139.

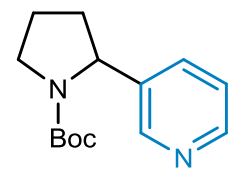

51

tert-butyl 2-(pyridin-3-yl)pyrrolidine-1-carboxylate (51): According to the general procedure, tert-butyl 2-(hydroxymethyl)pyrrolidine-1-carboxylate $(80.5 \mathrm{mg}, 0.4 \mathrm{mmol}$, 2.0 equiv.), 3-bromopyridine (31.6 mg, $0.2 \mathrm{mmol}, 1.0$ equiv.), $\mathrm{Ni}(\mathrm{dMebpy})\left(\mathrm{H}_{2} \mathrm{O}\right)_{4} \mathrm{Cl}_{2}$ (0.8 mg, 0.002 mmol, 0.01 equiv.), DPA (0.7 mg, $0.002 \mathrm{mmol}, 0.01$ equiv.), L4 (20.0 mg, 0.07 mmol, 0.35 equiv.), $\mathrm{CeCl}_{3}$ (4.9 mg, 0.02 mmol, 0.1 equiv.), $\mathrm{Na}_{3} \mathrm{PO}_{4}$ (98.4 mg, $0.6 \mathrm{mmol}, 3$ equiv.), $\mathrm{CH}_{3} \mathrm{CN}(2 \mathrm{~mL})$ and $\mathrm{DMSO}(0.25 \mathrm{~mL})$ were used. After $36 \mathrm{~h}$, the product was isolated by flash chromatography as a colorless oil (35 mg, 71\%). ${ }^{1} \mathbf{H}$ NMR 
(500 MHz, Chloroform- $\boldsymbol{d}) \delta 8.49$ (br, 2H), $7.68-7.48(\mathrm{~m}, 1 \mathrm{H}), 7.37-7.23(\mathrm{~m}, 1 \mathrm{H})$, $5.05-4.68(\mathrm{~m}, 1 \mathrm{H}), 3.73-3.47(\mathrm{~m}, 2 \mathrm{H}), 2.37$ (br, 1H), 1.87 (m, 3H), $1.44(\mathrm{~s}, 3 \mathrm{H}), 1.19$ (s, 6H). 13C NMR (125 MHz, Chloroform-d) $\delta$ 154.3, 147.6\&147.3, 146.6\&146.0, $140.7, \quad 134.7 \& 133.4, \quad 123.8 \& 123.4, \quad 79.7, \quad 59.1 \& 58.7, \quad 47.3 \& 47.1, \quad 35.9 \& 34.6$, 28.4\&28.1, 23.6\&23.3. HRMS (ESI+): calcd for $\mathrm{C}_{14} \mathrm{H}_{21} \mathrm{~N}_{2} \mathrm{O}_{2}(\mathrm{M}+\mathrm{H})$ 249.1598, found 249.1598 .

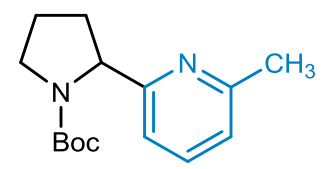

52

tert-butyl 2-(6-methylpyridin-2-yl)pyrrolidine-1-carboxylate (52): According to the general procedure, tert-butyl 2-(hydroxymethyl)pyrrolidine-1-carboxylate $(80.5 \mathrm{mg}$, $0.4 \mathrm{mmol}, 2.0$ equiv.), 2-bromo-6-methylpyridine (34.4 mg, $0.2 \mathrm{mmol}, 1.0$ equiv.), $\mathrm{Ni}(\mathrm{dMebpy})\left(\mathrm{H}_{2} \mathrm{O}\right)_{4} \mathrm{Cl}_{2}(0.8 \mathrm{mg}, 0.002 \mathrm{mmol}, 0.01$ equiv.), DPA (0.7 mg, $0.002 \mathrm{mmol}$, 0.01 equiv.), $\mathbf{L} 4$ (20.0 mg, $0.07 \mathrm{mmol}, 0.35$ equiv.), $\mathrm{CeCl}_{3}$ (4.9 mg, $0.02 \mathrm{mmol}, 0.1$ equiv.), $\mathrm{Na}_{3} \mathrm{PO}_{4}$ (98.4 mg, $0.6 \mathrm{mmol}, 3$ equiv.), $\mathrm{CH}_{3} \mathrm{CN}(2 \mathrm{~mL})$ and DMSO (0.25 mL) were used. After $36 \mathrm{~h}$, the product was isolated by flash chromatography as a colorless oil (32 mg, 61\%). ${ }^{\mathbf{1} H}$ NMR (500 MHz, Chloroform-d) $\delta 7.45$ (t, $\left.J=7.7 \mathrm{~Hz}, 1 \mathrm{H}\right), 6.90$ $(\mathrm{dd}, J=21.7,8.1 \mathrm{~Hz}, 2 \mathrm{H}), 4.86(\mathrm{~m}, 1 \mathrm{H}), 3.61-3.39(\mathrm{~m}, 2 \mathrm{H}), 2.46(\mathrm{~s}, 3 \mathrm{H}), 2.35-2.16$ (m, 1H), $2.00-1.87(\mathrm{~m}, 1 \mathrm{H}), 1.83-1.75(\mathrm{~m}, 2 \mathrm{H}), 1.39$ (s, 3H), $1.13(\mathrm{~s}, 6 \mathrm{H}) .{ }^{13} \mathbf{C}$ NMR (125 MHz, Chloroform-d) $\delta 163.2,157.4,154.6,136.1,121.1,116.3,79.3,62.8 \& 62.2$, 47.3\&47.0, 34.3\&33.1, 28.5\&28.2, 24.3, 23.5\&23.1. HRMS (ESI+): calcd for $\mathrm{C}_{15} \mathrm{H}_{23} \mathrm{~N}_{2} \mathrm{O}_{2}(\mathrm{M}+\mathrm{H})$ 263.1754, found 263.1750.

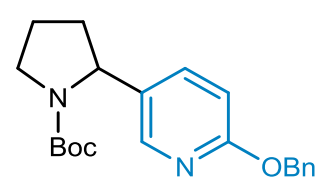

53 
tert-butyl 2-(6-(benzyloxy)pyridin-3-yl)pyrrolidine-1-carboxylate (53): According to the general procedure, tert-butyl 2-(hydroxymethyl)pyrrolidine-1-carboxylate (80.5 mg, 0.4 mmol, 2.0 equiv.), 2-(benzyloxy)-5-bromopyridine (52.8 mg, $0.2 \mathrm{mmol}, 1.0$ equiv.), $\mathrm{Ni}(\mathrm{dMebpy})\left(\mathrm{H}_{2} \mathrm{O}\right)_{4} \mathrm{Cl}_{2}$ (0.8 mg, $0.002 \mathrm{mmol}, 0.01$ equiv.), DPA (0.7 mg, 0.002 mmol, 0.01 equiv.), $\mathbf{L 4}$ (20.0 mg, $0.07 \mathrm{mmol}, 0.35$ equiv.), $\mathrm{CeCl}_{3}$ (4.9 mg, $0.02 \mathrm{mmol}$, 0.1 equiv.), $\mathrm{Na}_{3} \mathrm{PO}_{4}$ (98.4 mg, $0.6 \mathrm{mmol}, 3$ equiv.), $\mathrm{CH}_{3} \mathrm{CN}$ (2 mL) and DMSO (0.25 $\mathrm{mL}$ ) were used. After $36 \mathrm{~h}$, the product was isolated by flash chromatography as a colorless oil (43 mg, 61\%). ${ }^{\mathbf{1}} \mathbf{H}$ NMR (500 MHz, Chloroform-d) $\delta 8.01$ (s, 1H), 7.45 (t, $J=10.8 \mathrm{~Hz}, 3 \mathrm{H}), 7.37$ (t, $J=7.6 \mathrm{~Hz}, 2 \mathrm{H}), 7.32$ (d, $J=7.4 \mathrm{~Hz}, 1 \mathrm{H}), 6.77$ (d, $J=8.5$ $\mathrm{Hz}, 1 \mathrm{H}), 5.37$ (s, 2H), $4.82(\mathrm{~m}, 1 \mathrm{H}), 3.57$ (m, 2H), 2.31 (s, 1H), $1.97-1.78$ (m, 3H), 1.45 (s, 3H), 1.22 (s, 6H). ${ }^{13}$ C NMR (125 MHz, Chloroform-d) $\delta$ 162.6, 154.4, 144.3, 137.3, 136.4, 133.4, 128.4, 127.9, 127.8, 110.8, 79.5, 67.7, 58.6, 47.0, 35.8, 28.50\&28.2, 23.3. HRMS (ESI+): calcd for $\mathrm{C}_{21} \mathrm{H}_{27} \mathrm{~N}_{2} \mathrm{O}_{3}(\mathrm{M}+\mathrm{H})$ 355.2016, found 355.2021.

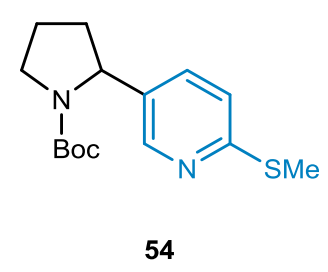

tert-butyl 2-(6-(methylthio)pyridin-3-yl)pyrrolidine-1-carboxylate (54): According to the general procedure, tert-butyl 2-(hydroxymethyl)pyrrolidine-1-carboxylate (80.5 mg, 0.4 mmol, 2.0 equiv.), 5-bromo-2-(methylthio)pyridine (40.8 mg, $0.2 \mathrm{mmol}, 1.0$ equiv.), $\mathrm{Ni}(\mathrm{dMebpy})\left(\mathrm{H}_{2} \mathrm{O}\right)_{4} \mathrm{Cl}_{2}(0.8 \mathrm{mg}, 0.002 \mathrm{mmol}, 0.01$ equiv.), DPA (0.7 mg, 0.002 mmol, 0.01 equiv.), $\mathbf{L 4}$ (20.0 mg, 0.07 mmol, 0.35 equiv.), $\mathrm{CeCl}_{3}$ (4.9 mg, $0.02 \mathrm{mmol}$, 0.1 equiv.), $\mathrm{Na}_{3} \mathrm{PO}_{4}$ (98.4 mg, $0.6 \mathrm{mmol}, 3$ equiv.), $\mathrm{CH}_{3} \mathrm{CN}$ (2 mL) and DMSO (0.25 $\mathrm{mL}$ ) were used. After $36 \mathrm{~h}$, the product was isolated by flash chromatography as a white solid (39 mg, 66\%). ${ }^{1}$ H NMR (500 MHz, Chloroform-d) $\delta 8.43-8.16$ (m, 1H), 7.33 $(\mathrm{d}, J=9.0 \mathrm{~Hz}, 1 \mathrm{H}), 7.13(\mathrm{~d}, J=8.3 \mathrm{~Hz}, 1 \mathrm{H}), 4.99-4.64(\mathrm{~m}, 1 \mathrm{H}), 3.71-3.41(\mathrm{~m}, 2 \mathrm{H})$, $2.55(\mathrm{~s}, 3 \mathrm{H}), 2.42-2.21(\mathrm{~m}, 1 \mathrm{H}), 1.90(\mathrm{~m}, 2 \mathrm{H}), 1.79(\mathrm{~m}, 1 \mathrm{H}), 1.44(\mathrm{~s}, 3 \mathrm{H}), 1.23(\mathrm{~s}, 6 \mathrm{H})$. 
${ }^{13}$ C NMR (125 MHz, Chloroform-d) $\delta$ 158.1, 154.4, 147.3, 121.3, 121.1, 79.7, 58.8\& 58.4, 47.0, 35.8\&34.7, 28.5\&28.3, 23.6\&23.2, 13.6. HRMS (ESI+): calcd for $\mathrm{C}_{15} \mathrm{H}_{23} \mathrm{~N}_{2} \mathrm{O}_{2} \mathrm{~S}(\mathrm{M}+\mathrm{H})$ 295.1475, found 295.1476.<smiles>O=C(c1ccccc1)N1CCCC1/C=C/c1ccccc1</smiles>

$55, E / Z=4: 1$<smiles>O=C(c1ccccc1)N1CCCC1/C=C\P</smiles>

Boc

tert-butyl 2-styrylpyrrolidine-1-carboxylate (55) According to the general procedure, tert-butyl 2-(hydroxymethyl)pyrrolidine-1-carboxylate ( $80.5 \mathrm{mg}, 0.4 \mathrm{mmol}, 2.0$ equiv.), (E)-(2-bromovinyl)benzene (36.6 mg, $0.2 \mathrm{mmol}, 1.0$ equiv.), $\mathrm{Ni}(\mathrm{dMebpy})\left(\mathrm{H}_{2} \mathrm{O}\right)_{4} \mathrm{Cl}_{2}$ (0.8 mg, 0.002 mmol, 0.01 equiv.), DPA (0.7 mg, 0.002 mmol, 0.01 equiv.), L4 (20.0 mg, 0.07 mmol, 0.35 equiv.), $\mathrm{CeCl}_{3}$ (4.9 mg, 0.02 mmol, 0.1 equiv.), $\mathrm{Na}_{3} \mathrm{PO}_{4}$ (98.4 mg, $0.6 \mathrm{mmol}, 3$ equiv.), $\mathrm{CH}_{3} \mathrm{CN}(2 \mathrm{~mL})$ and DMSO $(0.25 \mathrm{~mL})$ were used. After $36 \mathrm{~h}$, the product was isolated by flash chromatography as a colorless oil $(36 \mathrm{mg}, 66 \%, \mathrm{E} / \mathrm{Z}=$ 4:1). ${ }^{1} \mathrm{H}$ NMR (500 MHz, Chloroform-d) $\delta(E / Z=4: 1) 7.39-7.22(\mathrm{~m}, 5 \mathrm{H}), 6.50-$ $6.31(\mathrm{~m}, 1 \mathrm{H}), 6.11(\mathrm{dd}, J=16.2,6.5 \mathrm{~Hz}, 0.8 \mathrm{H}), 5.63(\mathrm{~m}, 0.2 \mathrm{H}), 4.85-4.31(\mathrm{~m}, 1 \mathrm{H})$, $3.58-3.35(\mathrm{~m}, 2 \mathrm{H}), 2.26-2.01(\mathrm{~m}, 1 \mathrm{H}), 2.01-1.74(\mathrm{~m}, 3 \mathrm{H}), 1.45(\mathrm{~s}, 9 \mathrm{H}) .{ }^{1} \mathbf{H}$ NMR (400 MHz, Chloroform- $d$ ) $\delta$ (E-isomer) 7.31 (m, 5H), 6.42 (d, $J=15.7 \mathrm{~Hz}, 1 \mathrm{H}), 6.11$ $(\mathrm{dd}, J=15.9,6.4 \mathrm{~Hz}, 1 \mathrm{H}), 4.45(\mathrm{~s}, 1 \mathrm{H}), 3.48(\mathrm{~m}, 2 \mathrm{H}), 2.12(\mathrm{~m}, 1 \mathrm{H}), 2.04-1.75(\mathrm{~m}$, 3H), 1.45 (s, 9H). ${ }^{13} \mathbf{C}$ NMR (100 MHz, Chloroform-d) $\delta$ (E-isomer) 154.6, 137.1, 130.7, 129.4, 128.5, 127.2, 126.3, 79.2, 58.9, 46.3, 32.5, 28.5, 23.5. HRMS (ESI+): calcd for $\mathrm{C}_{17} \mathrm{H}_{23} \mathrm{NO}_{2} \mathrm{Na}(\mathrm{M}+\mathrm{Na}) 296.1621$, found 296.1627 . 


\section{Spectral Data}

${ }^{1} \mathrm{H}$ NMR $500 \mathrm{MHz}, \mathrm{CDCl}_{3}$

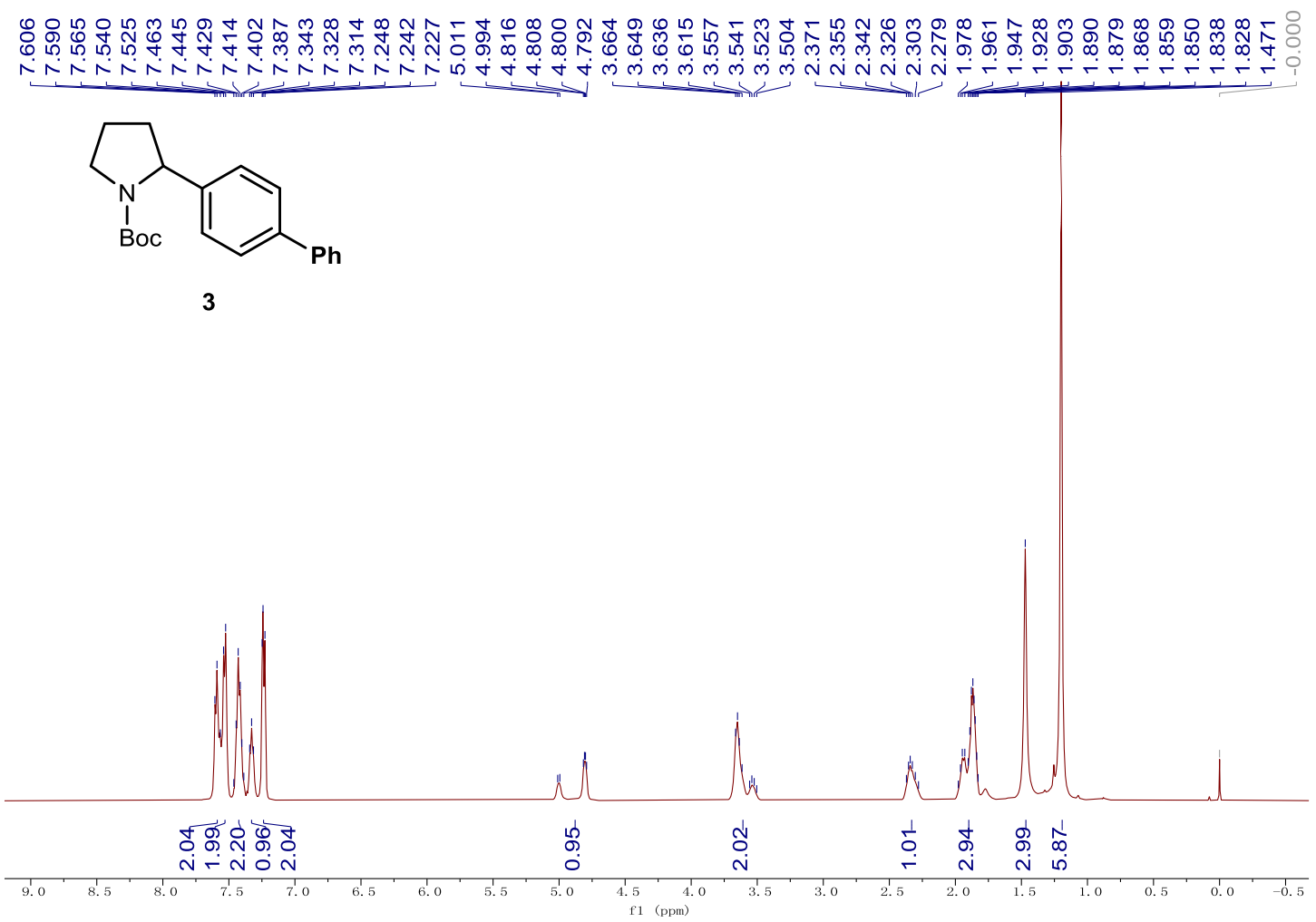

${ }^{13} \mathrm{C}$ NMR $125 \mathrm{MHz}, \mathrm{CDCl}_{3}$

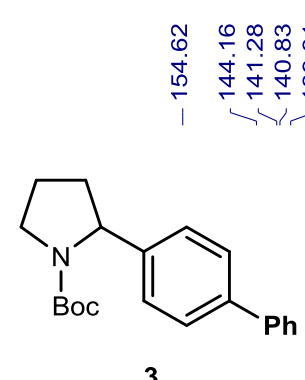

ஸे ำ

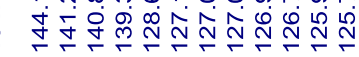
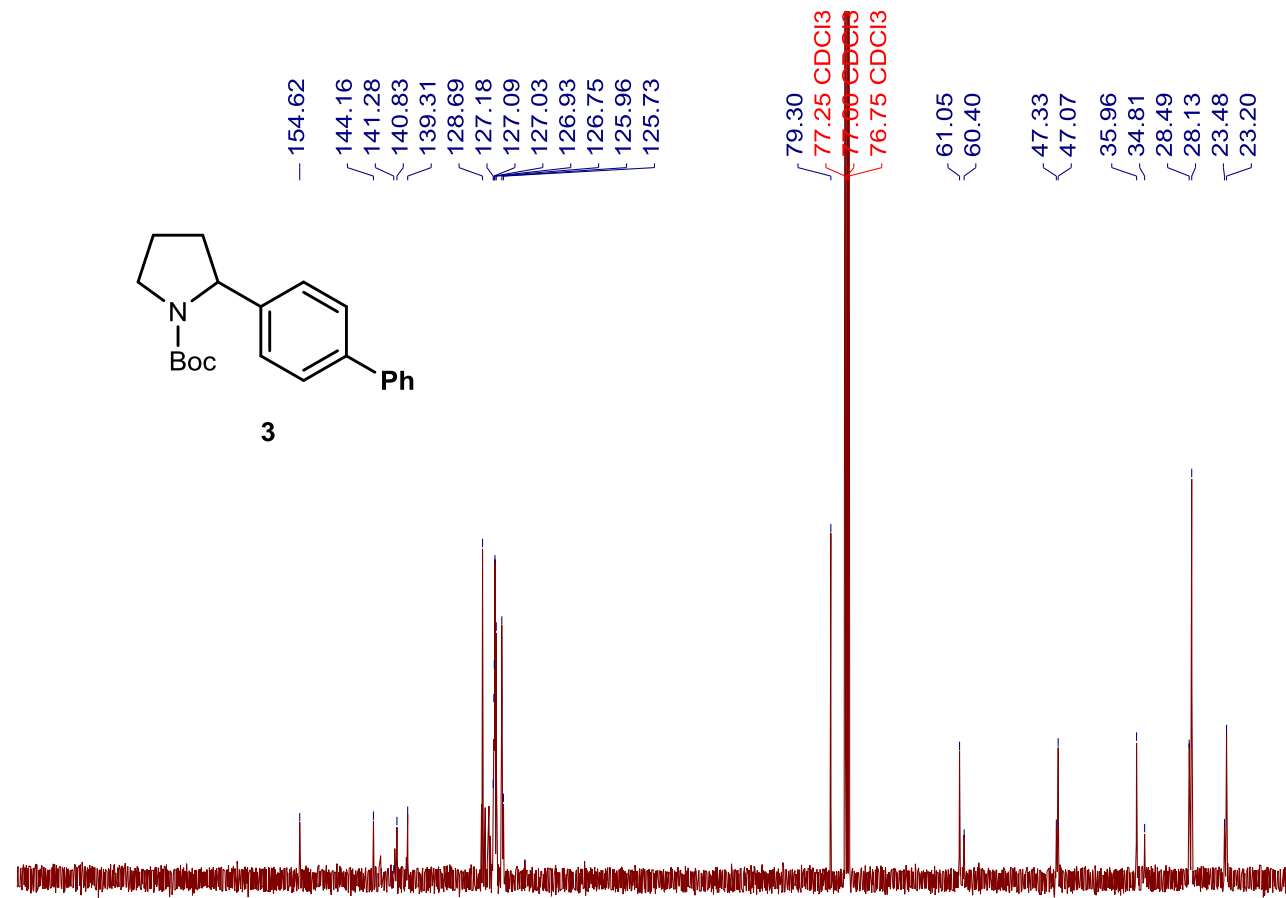

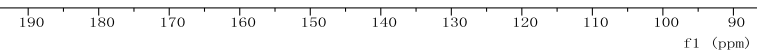


${ }^{1} \mathrm{H}$ NMR $500 \mathrm{MHz}, \mathrm{CDCl}_{3}$

언

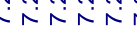

(1)
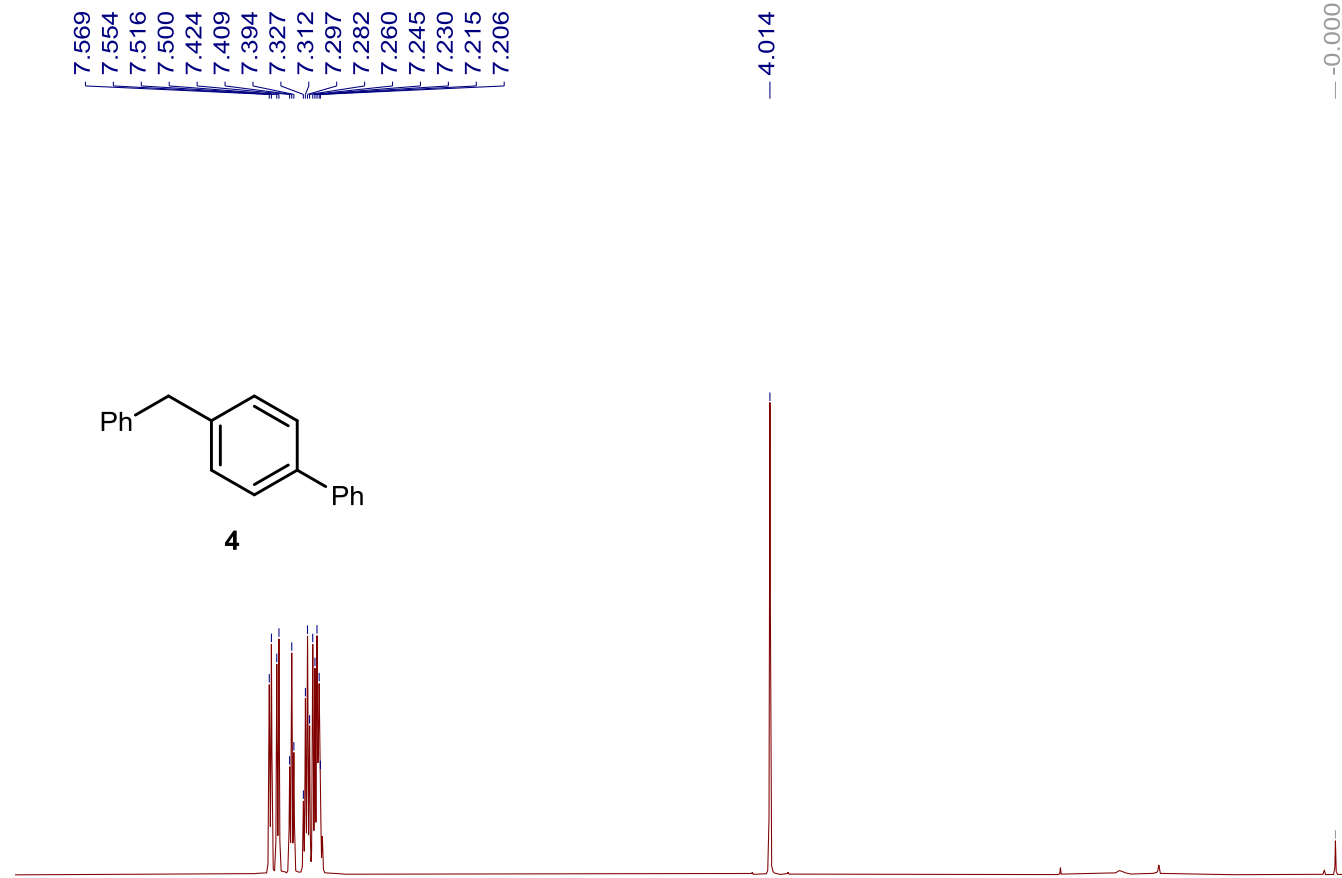

상요

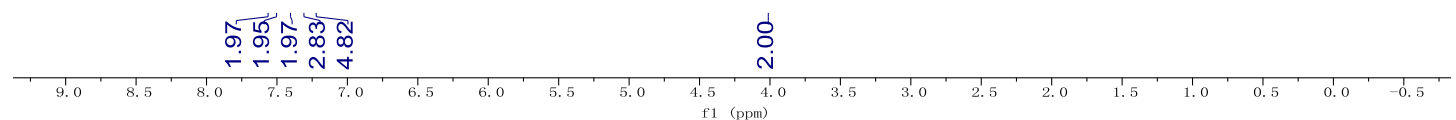

${ }^{13} \mathrm{C}$ NMR $125 \mathrm{MHz}, \mathrm{CDCl}_{3}$
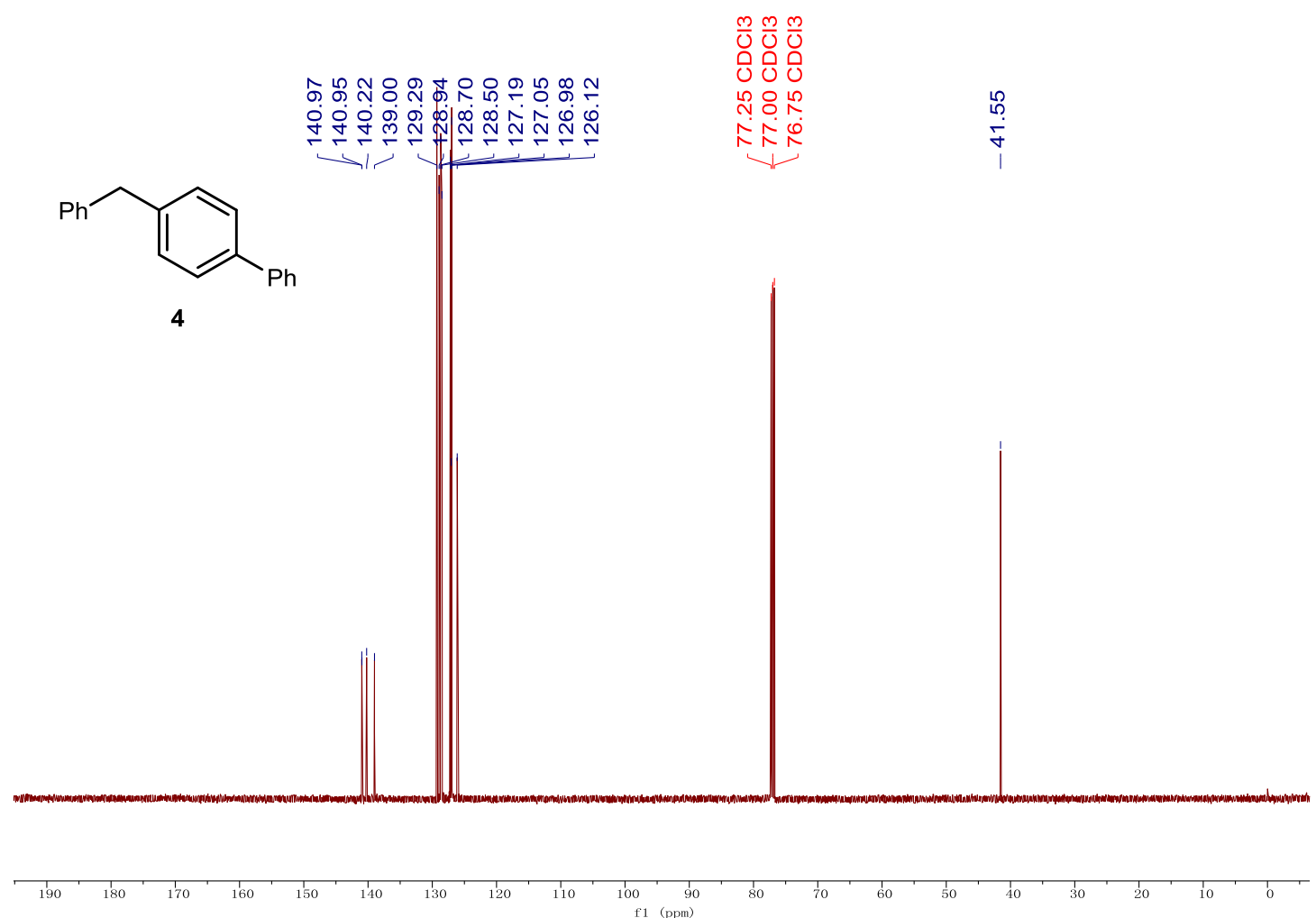
${ }^{1} \mathrm{H}$ NMR $500 \mathrm{MHz}, \mathrm{CDCl}_{3}$

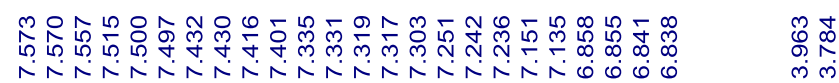

-5
$\overline{8}$
0
0
0
0

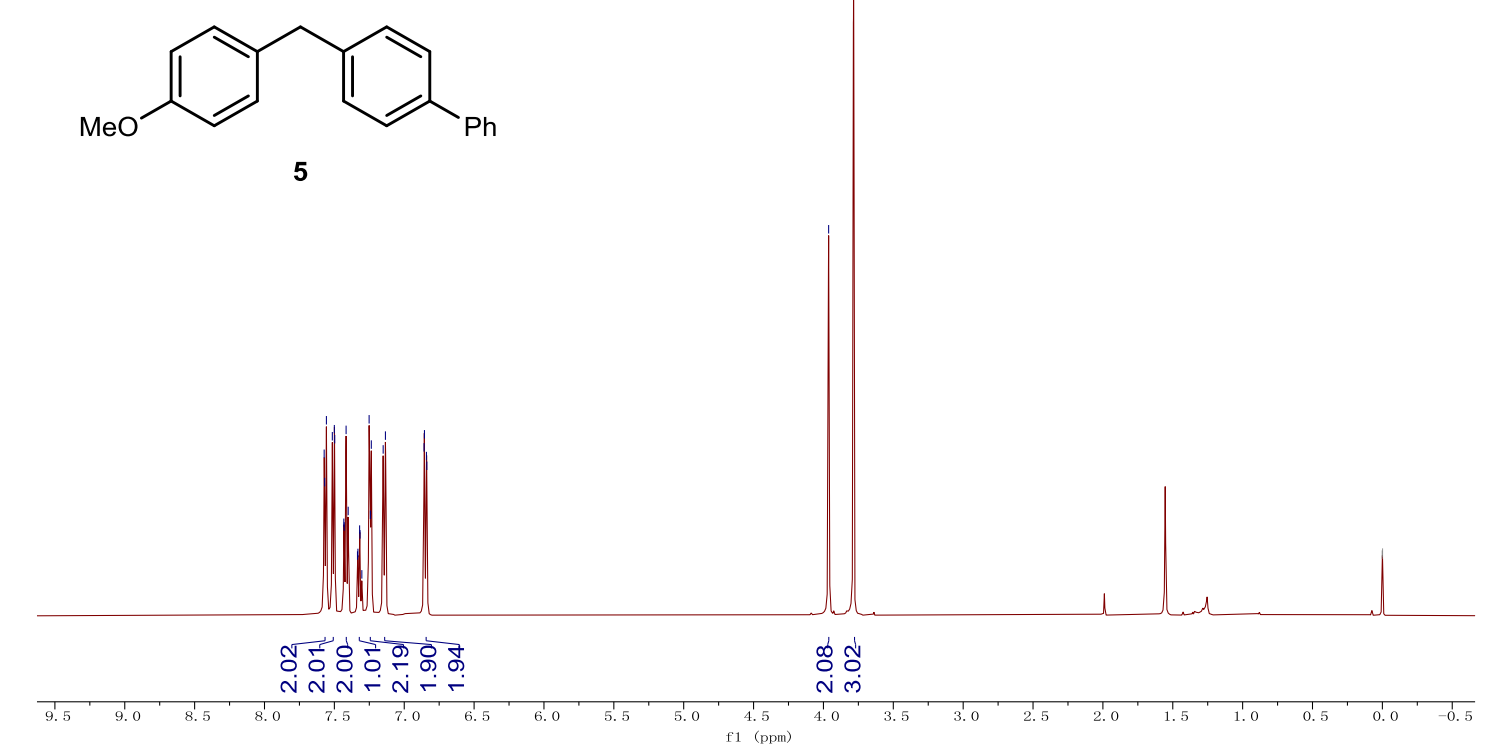

5

${ }^{13} \mathrm{C}$ NMR $125 \mathrm{MHz}, \mathrm{CDCl}_{3}$
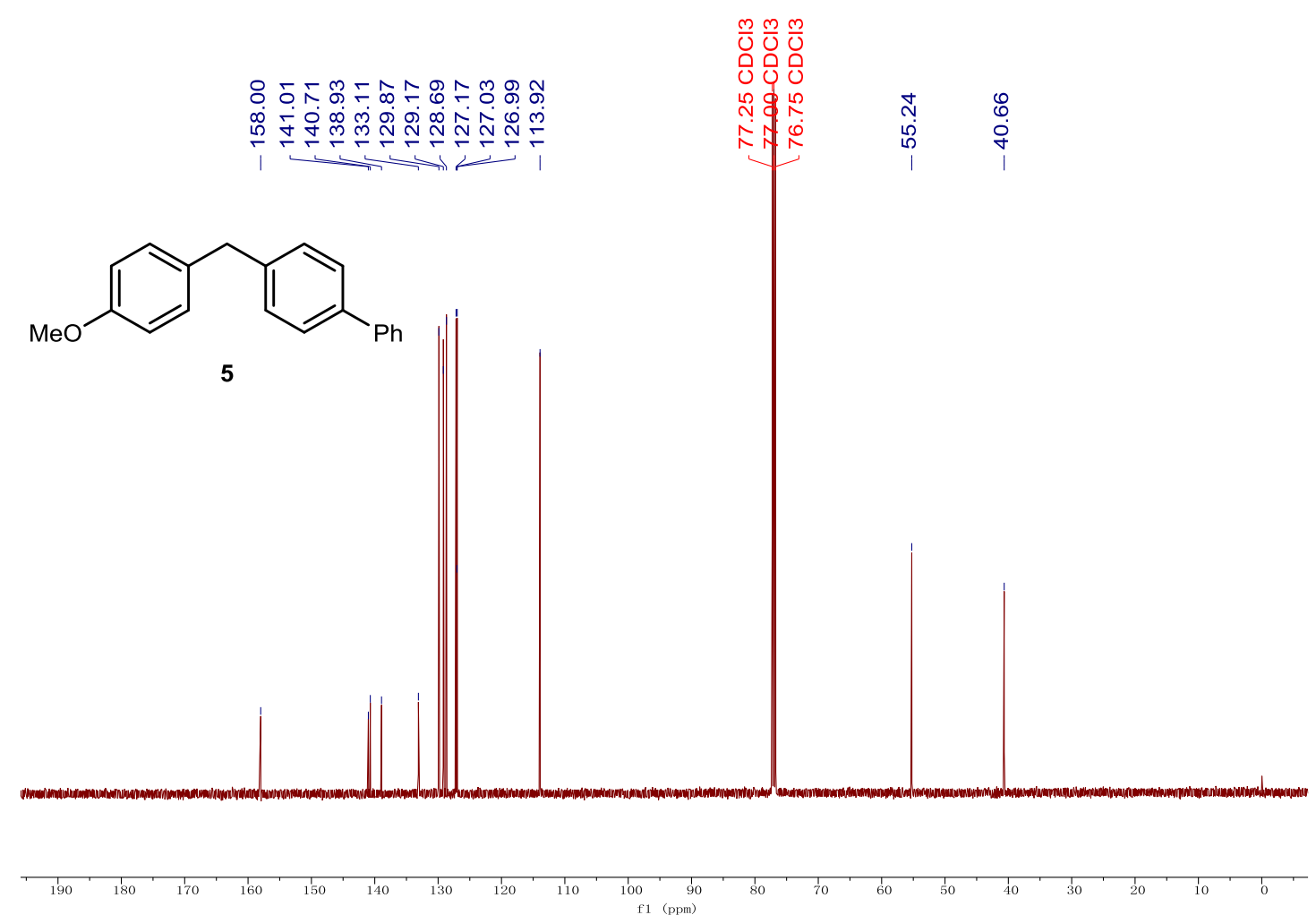
${ }^{1} \mathrm{H}$ NMR $500 \mathrm{MHz}, \mathrm{CDCl}_{3}$

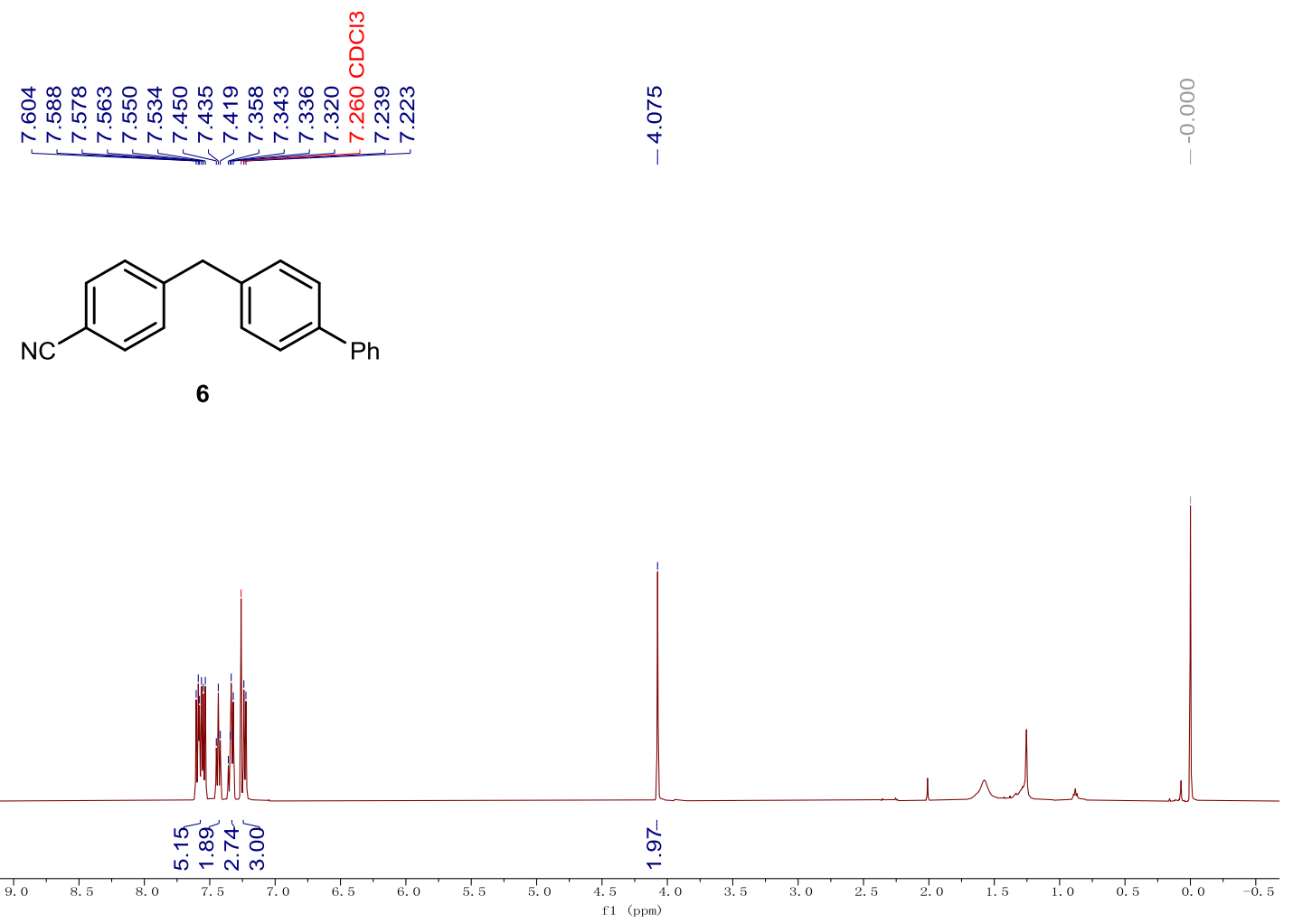

${ }^{13} \mathrm{C}$ NMR $125 \mathrm{MHz}, \mathrm{CDCl}_{3}$
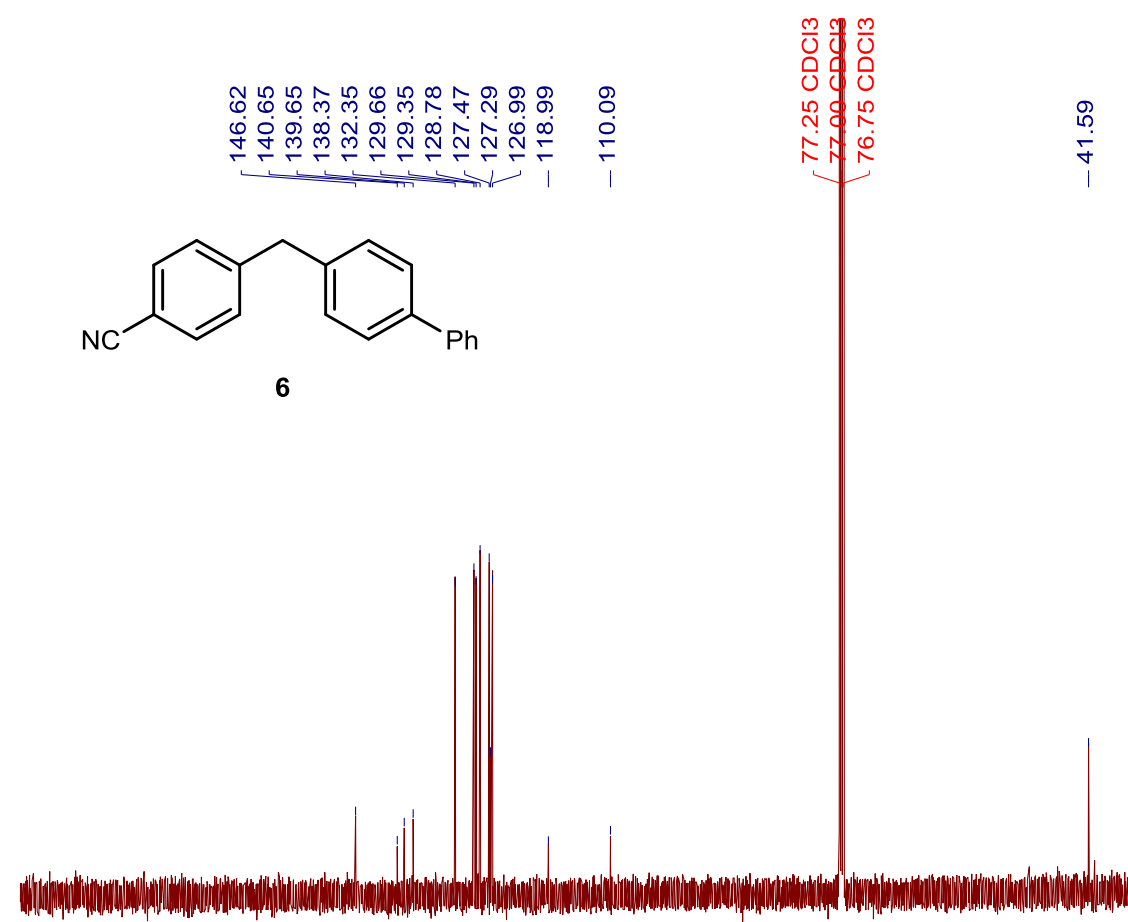

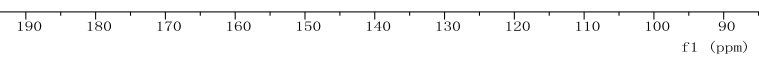


${ }^{1} \mathrm{H}$ NMR $500 \mathrm{MHz}, \mathrm{CDCl}_{3}$

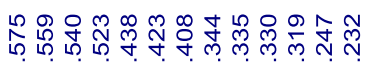

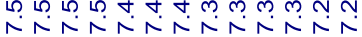<smiles>FC(F)(F)c1ccc(Cc2ccc(-c3ccccc3)cc2)cc1</smiles>

7

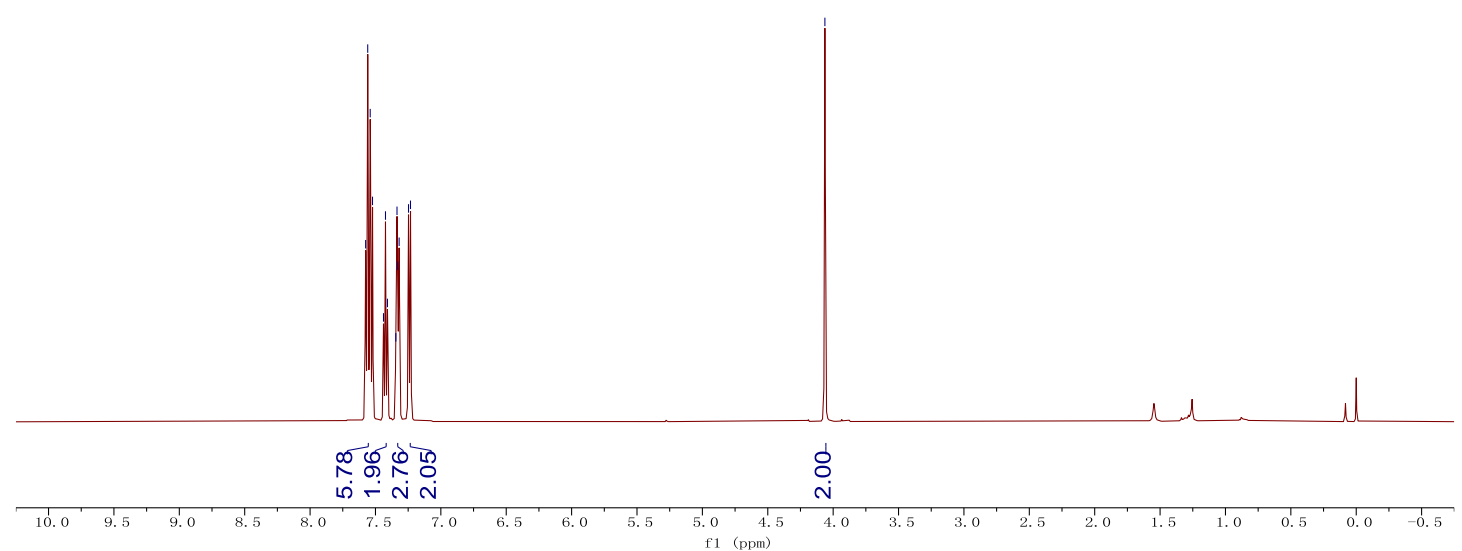

${ }^{13} \mathrm{C}$ NMR $125 \mathrm{MHz}, \mathrm{CDCl}_{3}$
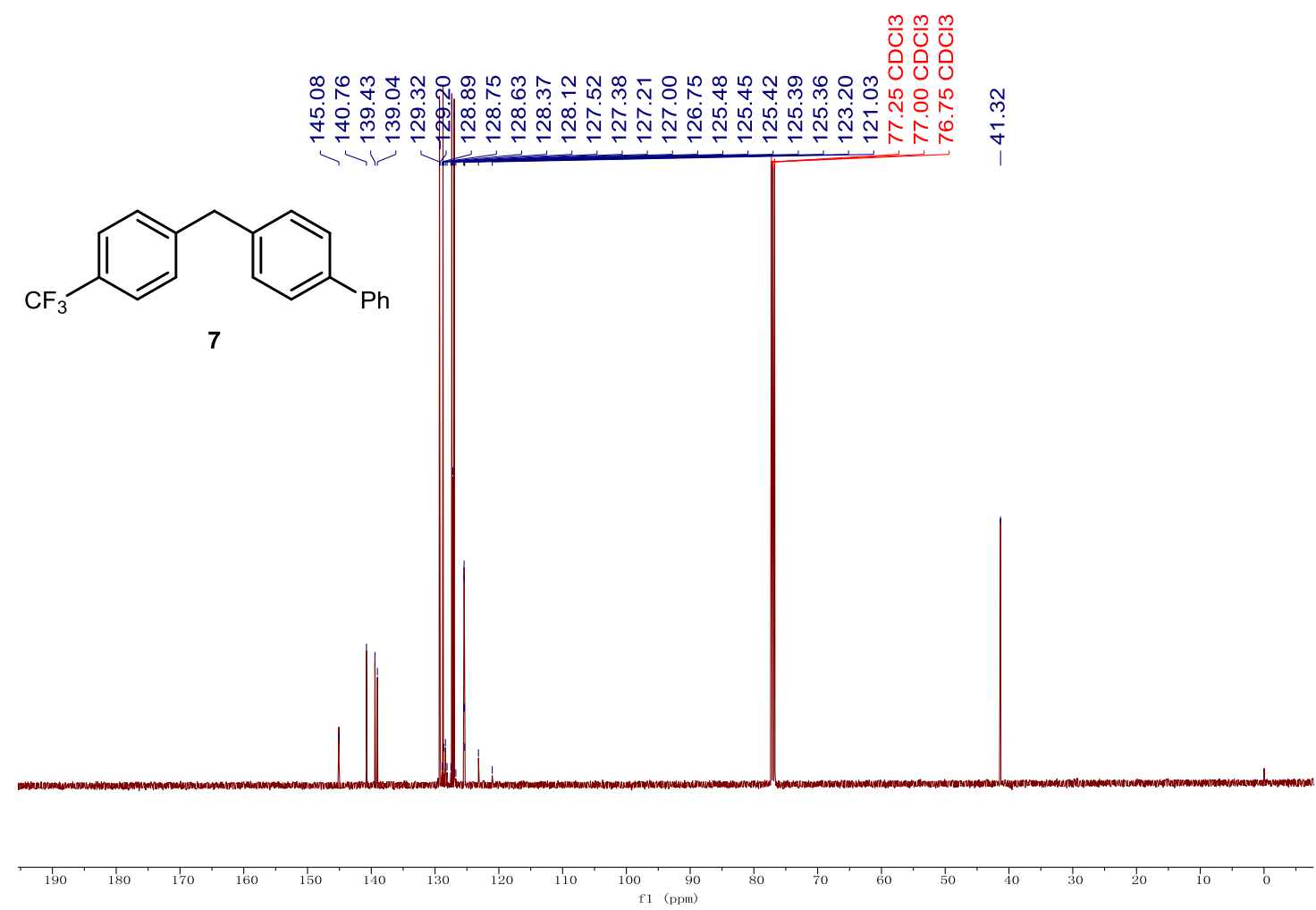
${ }^{19} \mathrm{~F}$ NMR $470 \mathrm{MHz}, \mathrm{CDCl}_{3}$

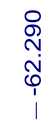<smiles>FC(F)(F)c1ccc(Cc2ccc(-c3ccccc3)cc2)cc1</smiles>

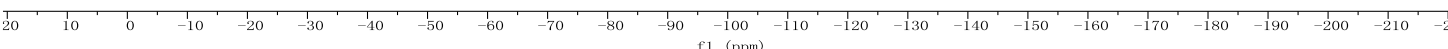


${ }^{1} \mathrm{H}$ NMR $500 \mathrm{MHz}, \mathrm{CDCl}_{3}$

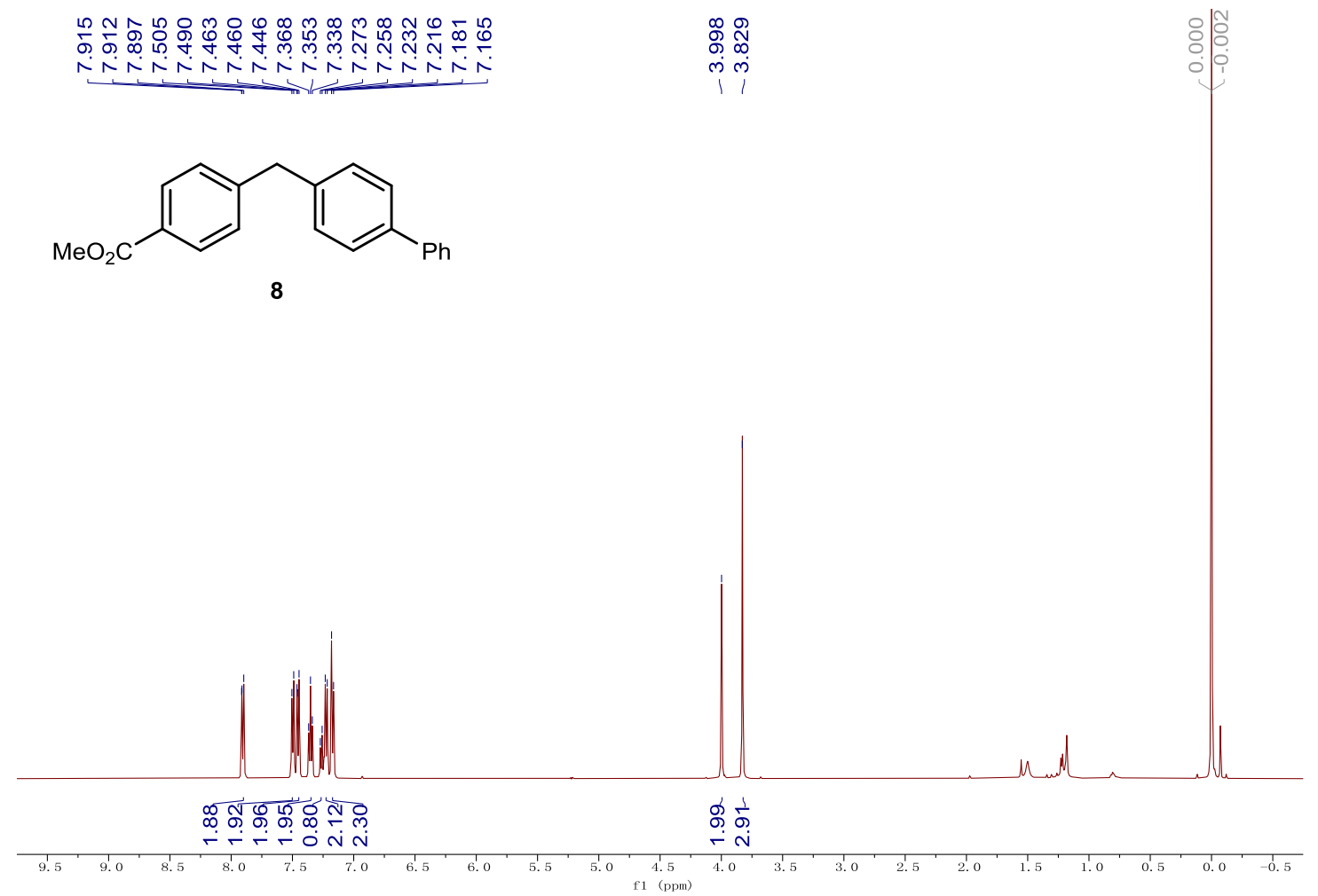

${ }^{13} \mathrm{C}$ NMR $125 \mathrm{MHz}, \mathrm{CDCl}_{3}$
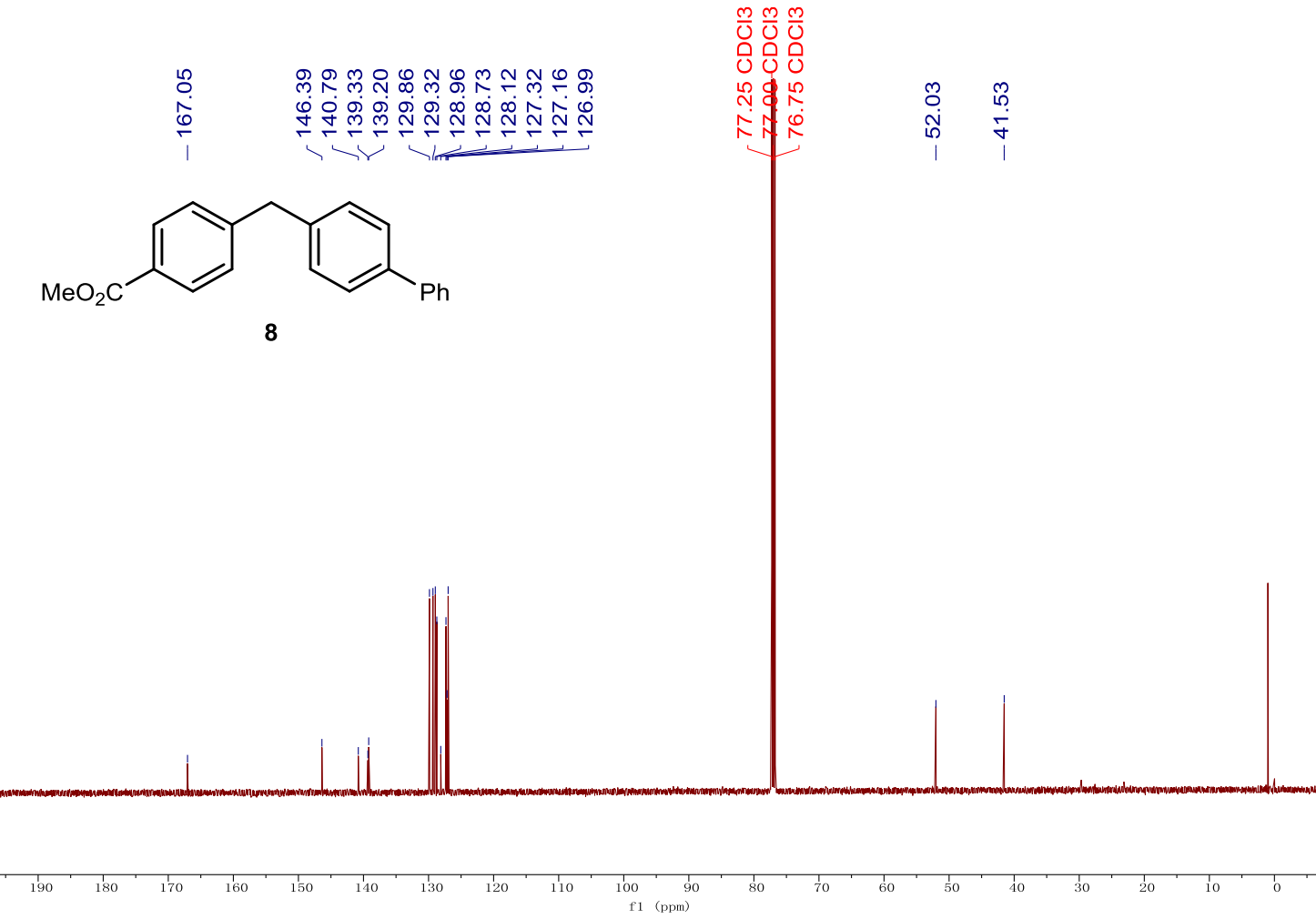
${ }^{1} \mathrm{H}$ NMR $500 \mathrm{MHz}, \mathrm{CDCl}_{3}$

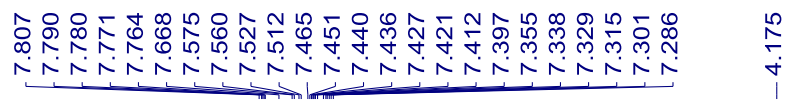<smiles>c1ccc(-c2ccc(Cc3ccc4ccccc4c3)cc2)cc1</smiles>

9

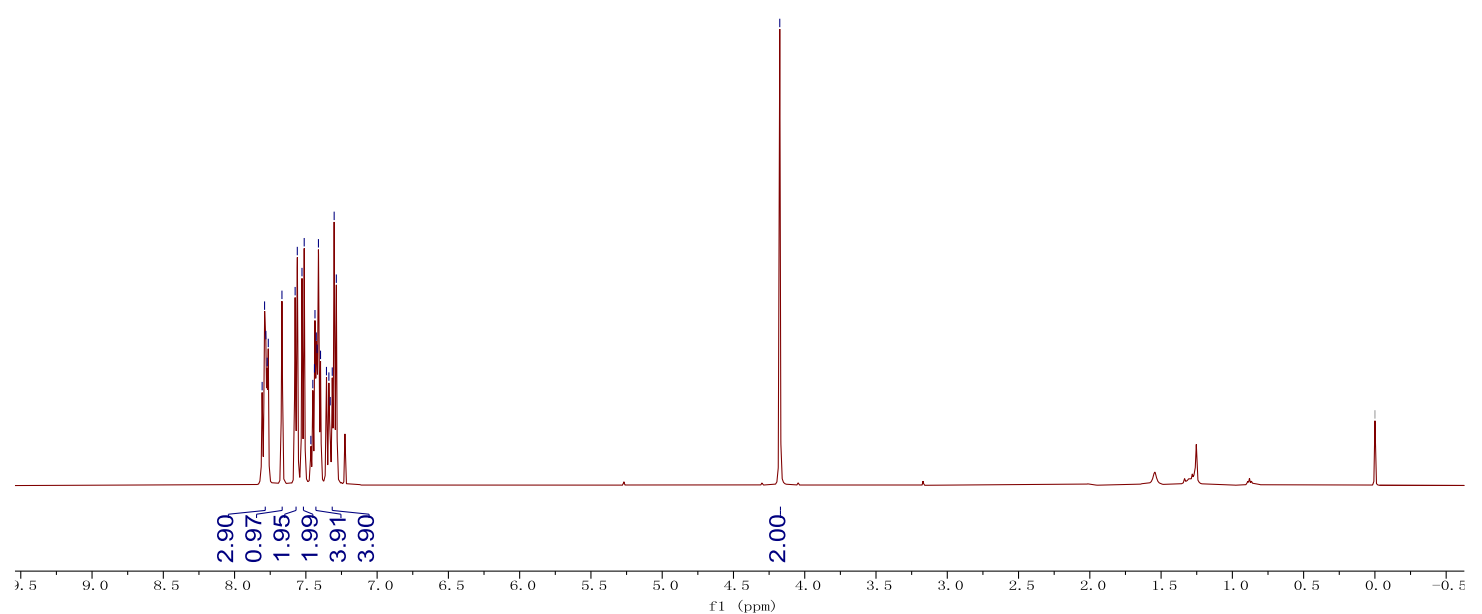

${ }^{13} \mathrm{C}$ NMR $125 \mathrm{MHz}, \mathrm{CDCl}_{3}$
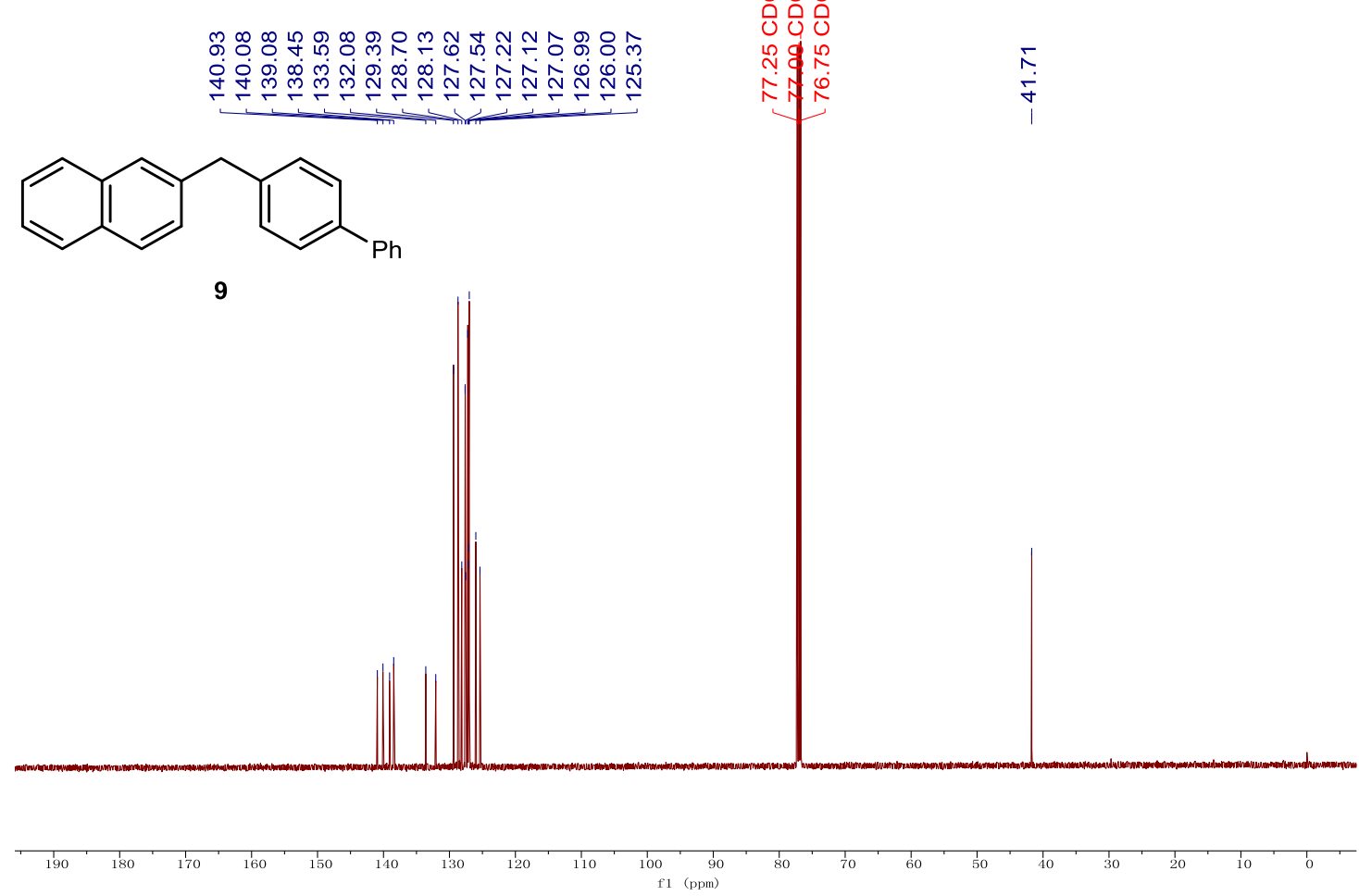
${ }^{1} \mathrm{H}$ NMR $500 \mathrm{MHz}, \mathrm{CDCl}_{3}$

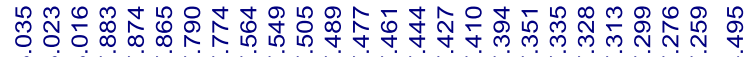

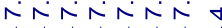<smiles>c1ccc(-c2ccc(Cc3cccc4ccccc34)cc2)cc1</smiles>

10

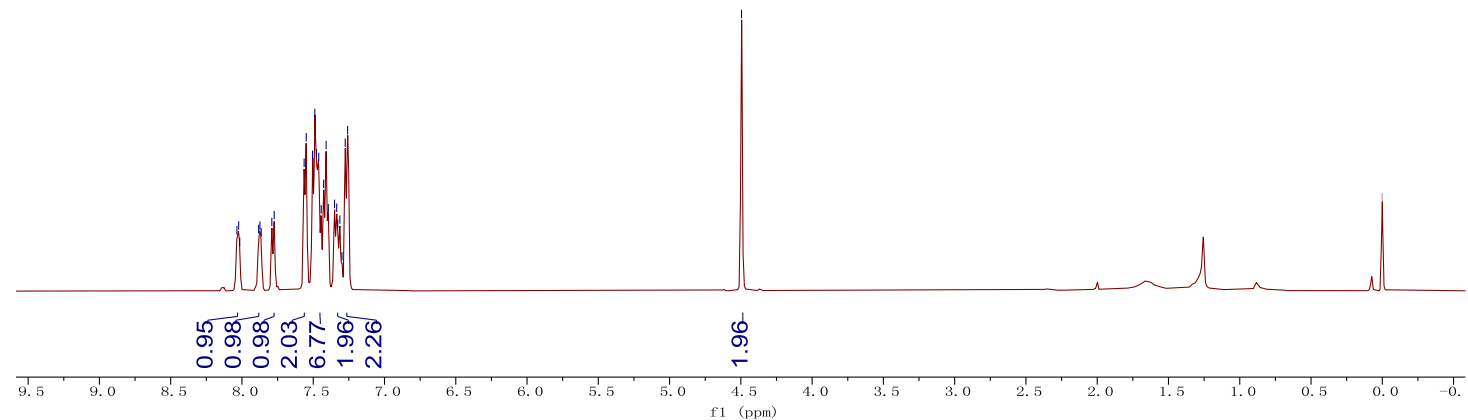

${ }^{13} \mathrm{C}$ NMR $125 \mathrm{MHz}, \mathrm{CDCl}_{3}$

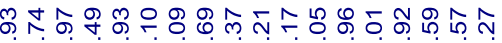

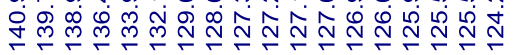<smiles>c1ccc(-c2ccc(Cc3cccc4ccccc34)cc2)cc1</smiles>

10
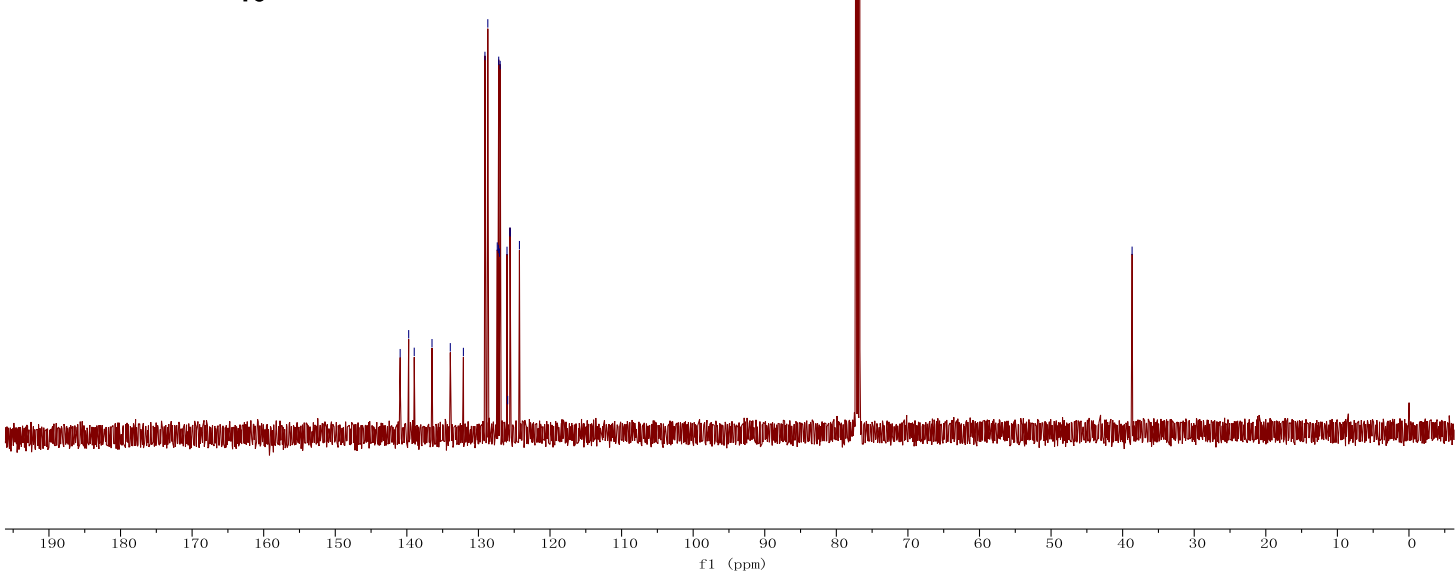
${ }^{1} \mathrm{H}$ NMR $500 \mathrm{MHz}, \mathrm{CDCl}_{3}$

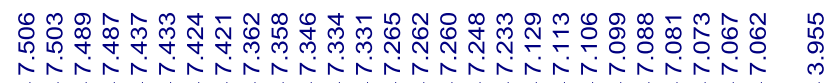

$\stackrel{\substack{n \\ \text { N }}}{\text { I }}$<smiles>Cc1ccccc1Cc1ccc(-c2ccccc2)cc1</smiles>

11

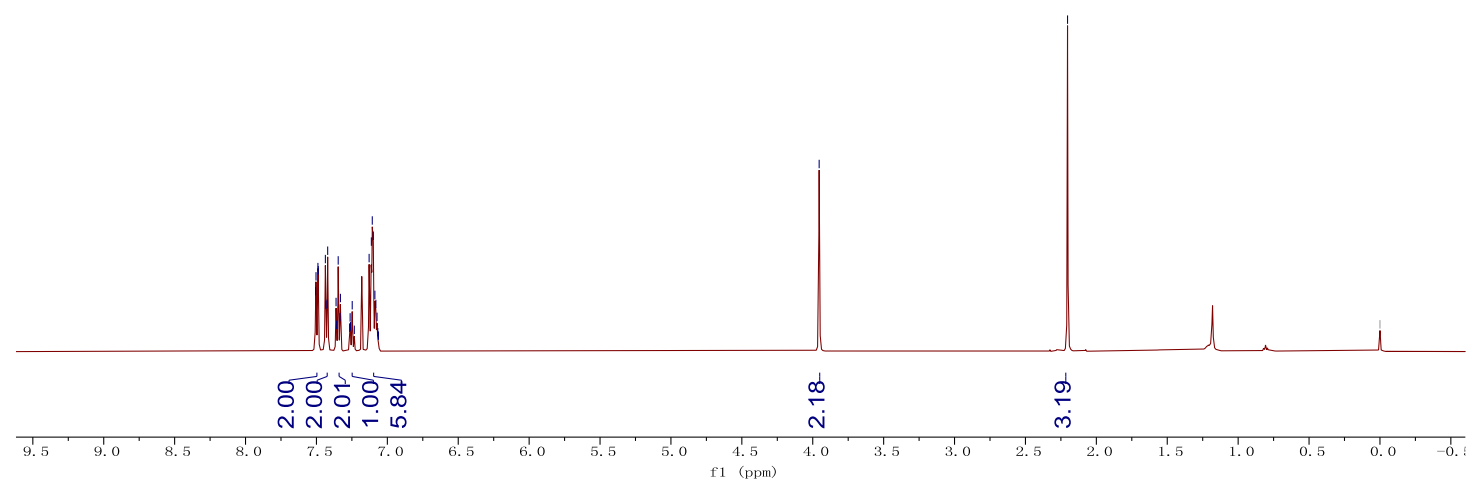

${ }^{13} \mathrm{C}$ NMR $125 \mathrm{MHz}, \mathrm{CDCl}_{3}$

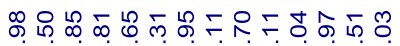

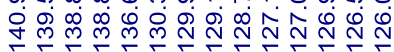

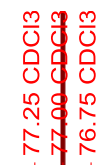

\begin{tabular}{ll}
$\hat{0}$ & \multicolumn{1}{c}{} \\
$\dot{0}$ & \\
1 & 1
\end{tabular}<smiles>Cc1ccccc1Cc1ccc(-c2ccccc2)cc1</smiles>

11
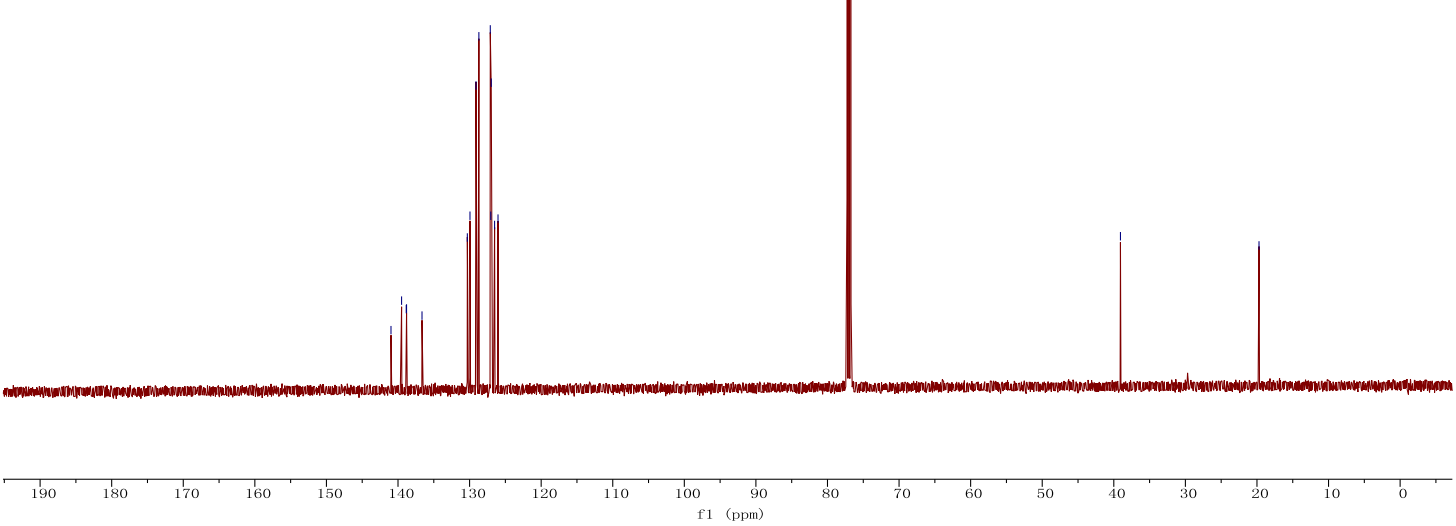
${ }^{1} \mathrm{H}$ NMR $500 \mathrm{MHz}, \mathrm{CDCl}_{3}$

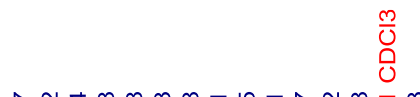

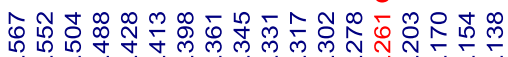

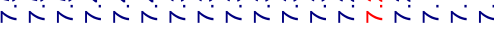

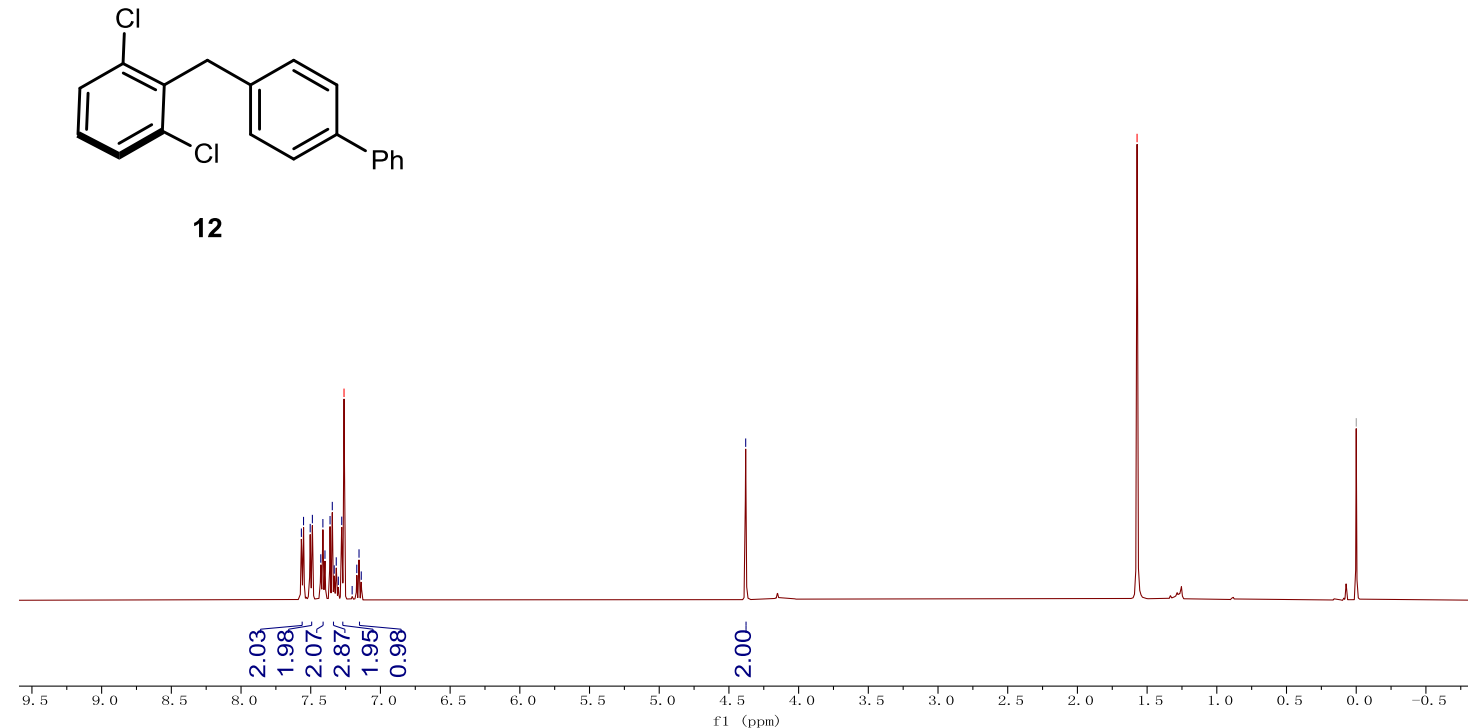

12

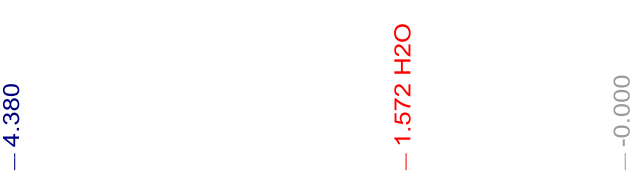

${ }^{13} \mathrm{C}$ NMR $125 \mathrm{MHz}, \mathrm{CDCl}_{3}$
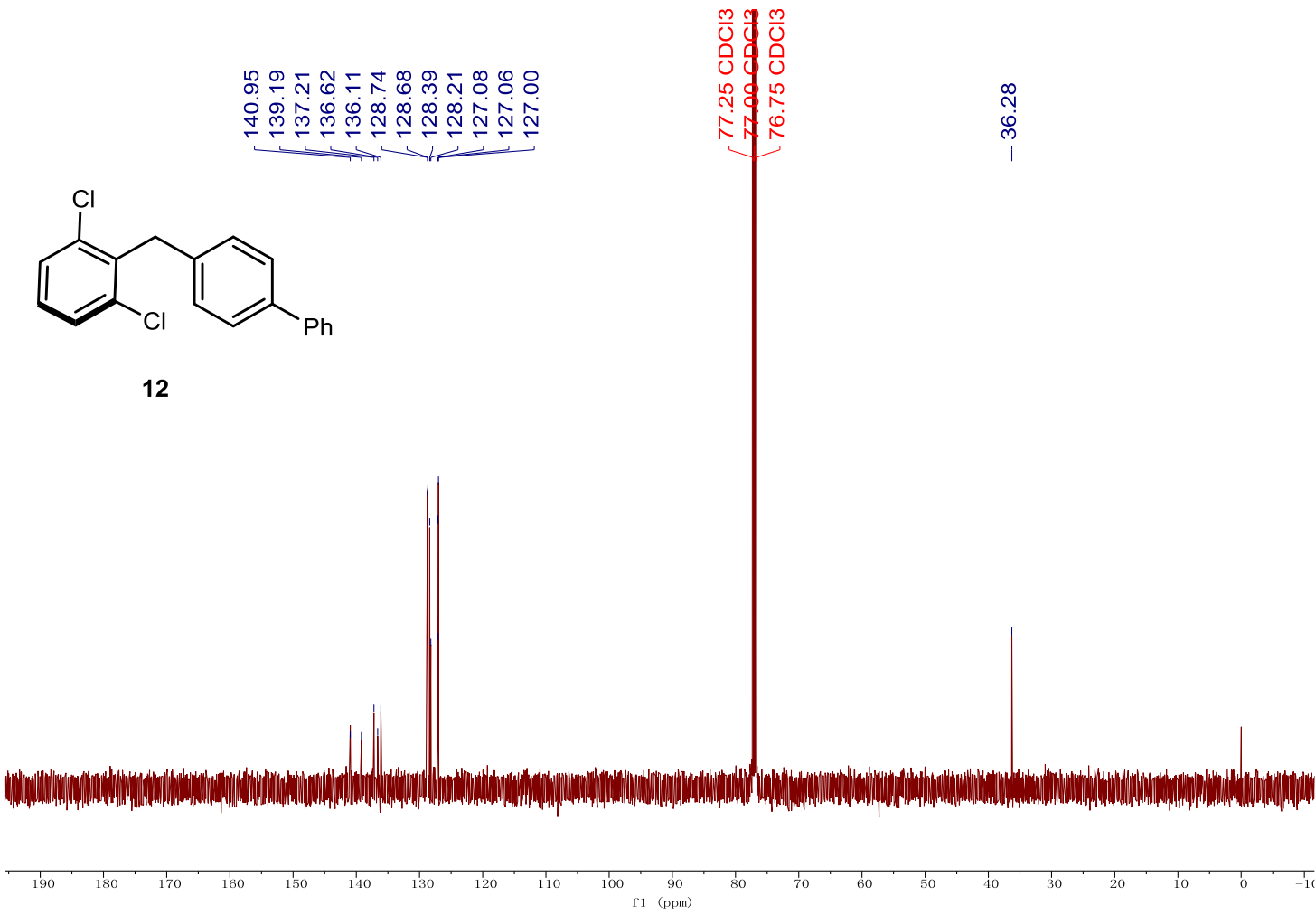
${ }^{1} \mathrm{H}$ NMR $500 \mathrm{MHz}, \mathrm{CDCl}_{3}$

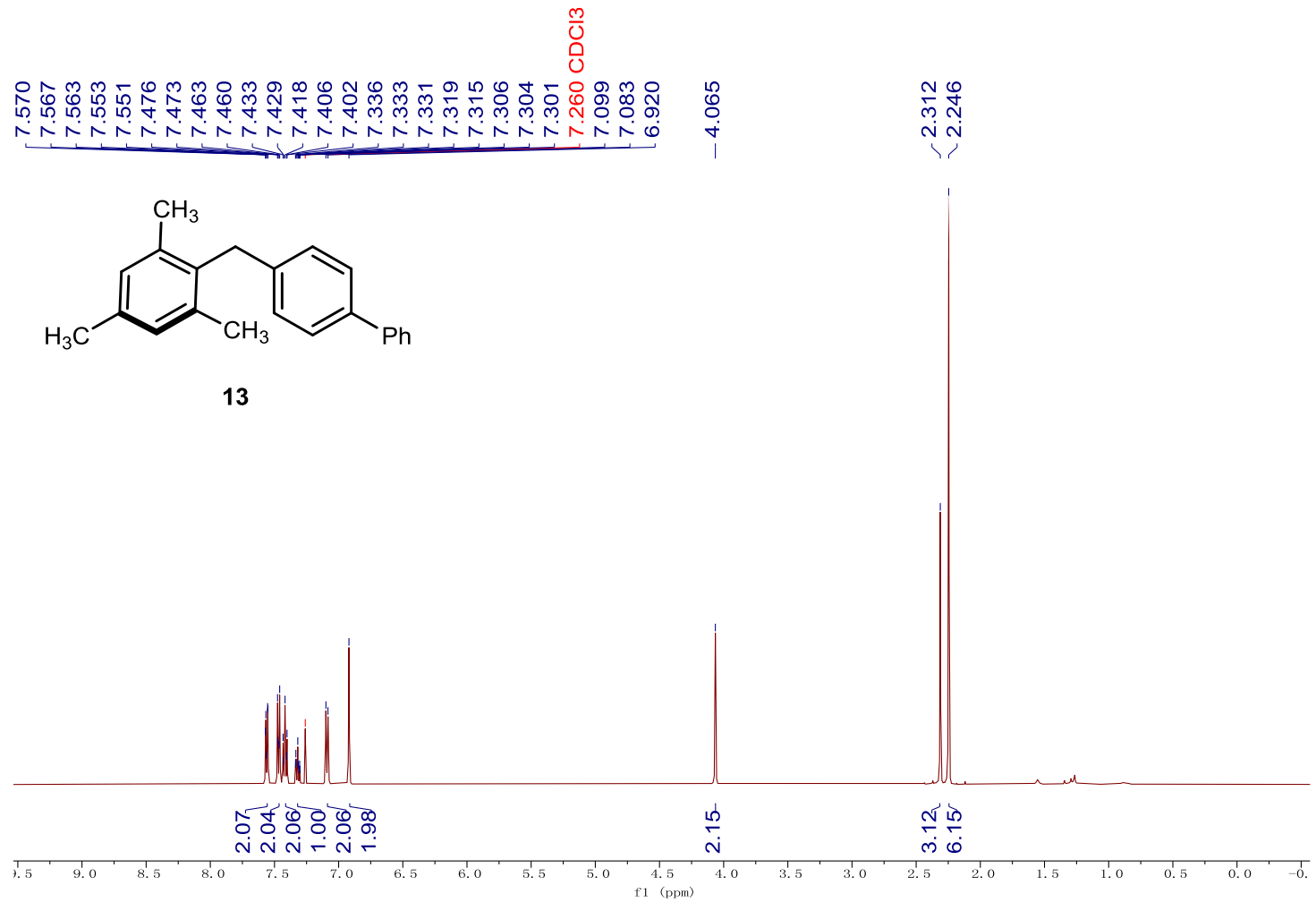

${ }^{13} \mathrm{C}$ NMR $125 \mathrm{MHz}, \mathrm{CDCl}_{3}$
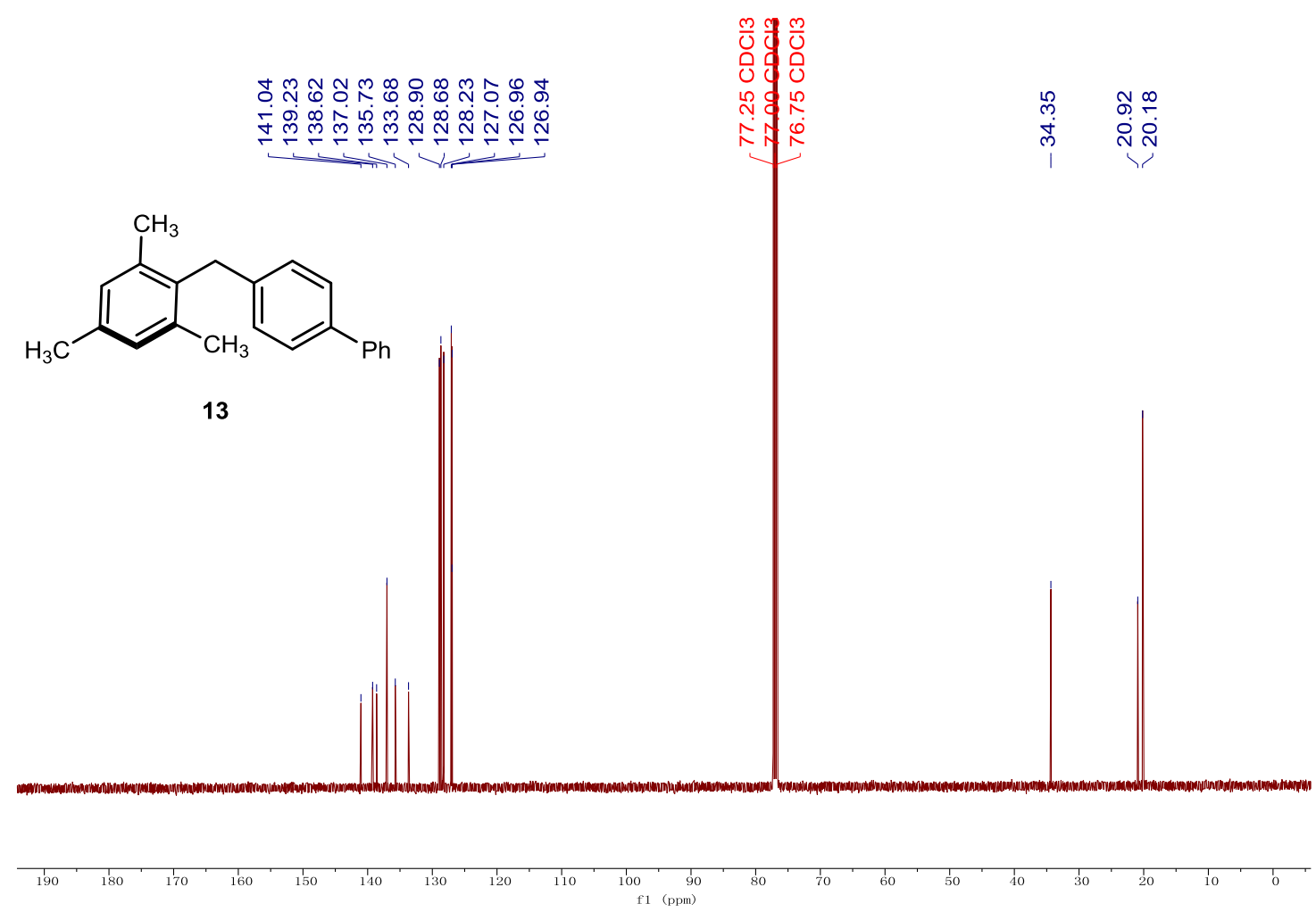
${ }^{1} \mathrm{H}$ NMR $500 \mathrm{MHz}, \mathrm{CDCl}_{3}$

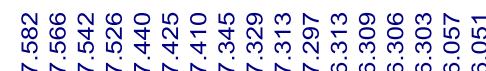

r r r r r T

$\underset{\substack{\infty \\ \leftarrow}}{+}$<smiles>c1ccc(-c2ccc(Cc3ccco3)cc2)cc1</smiles>

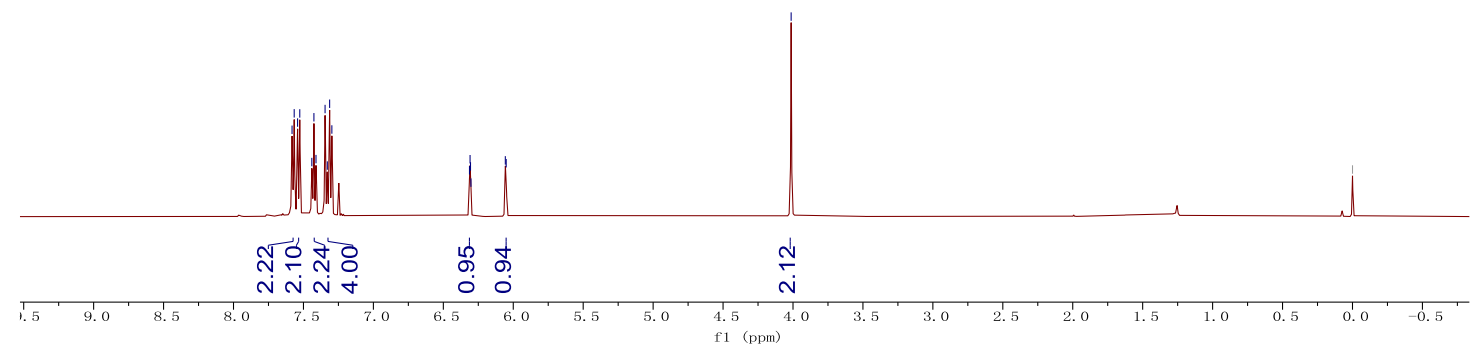

${ }^{13} \mathrm{C}$ NMR $125 \mathrm{MHz}, \mathrm{CDCl}_{3}$
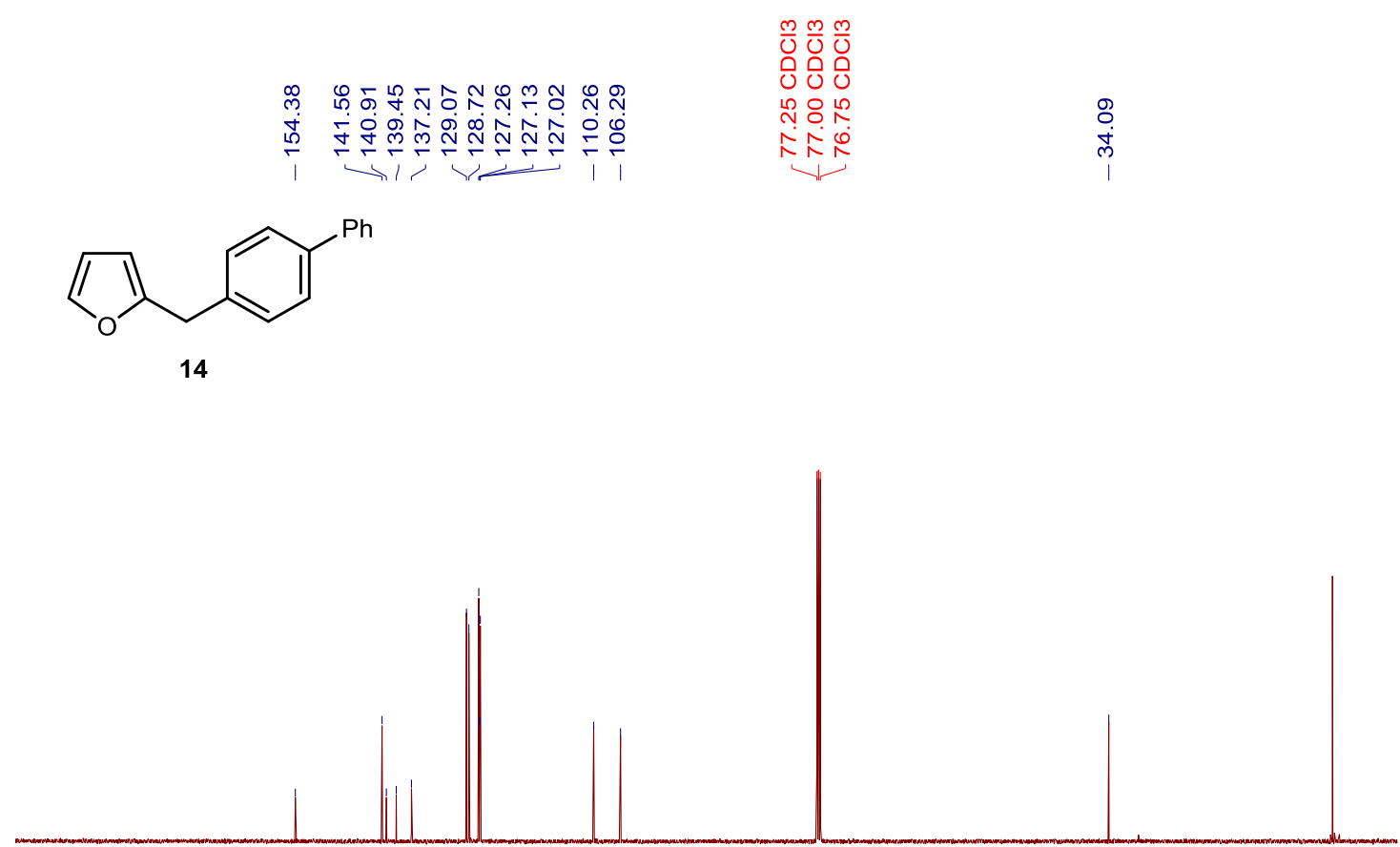

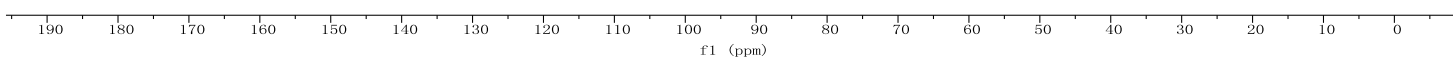


${ }^{1} \mathrm{H}$ NMR $500 \mathrm{MHz}, \mathrm{CDCl}_{3}$
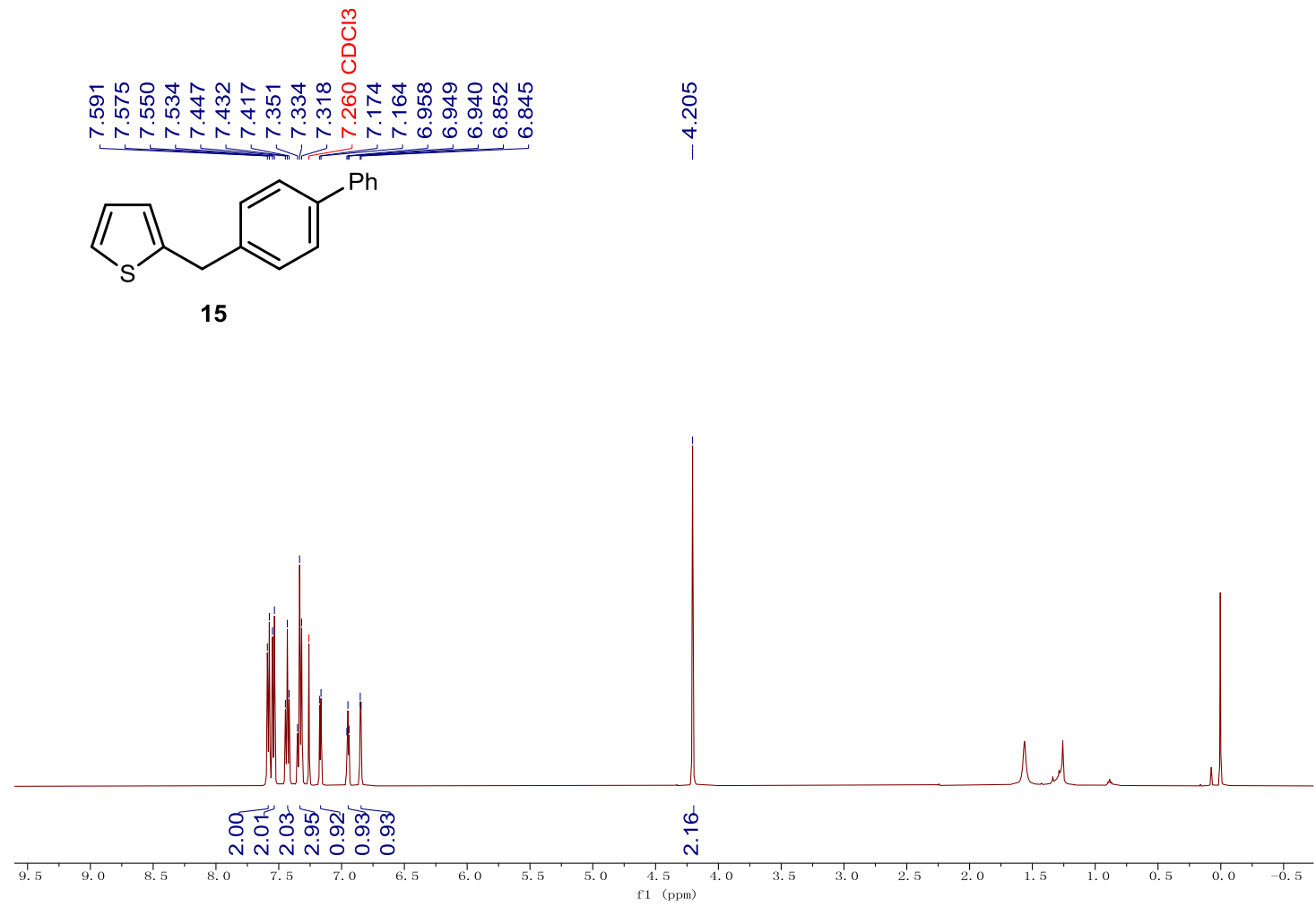

${ }^{13} \mathrm{C}$ NMR $125 \mathrm{MHz}, \mathrm{CDCl}_{3}$
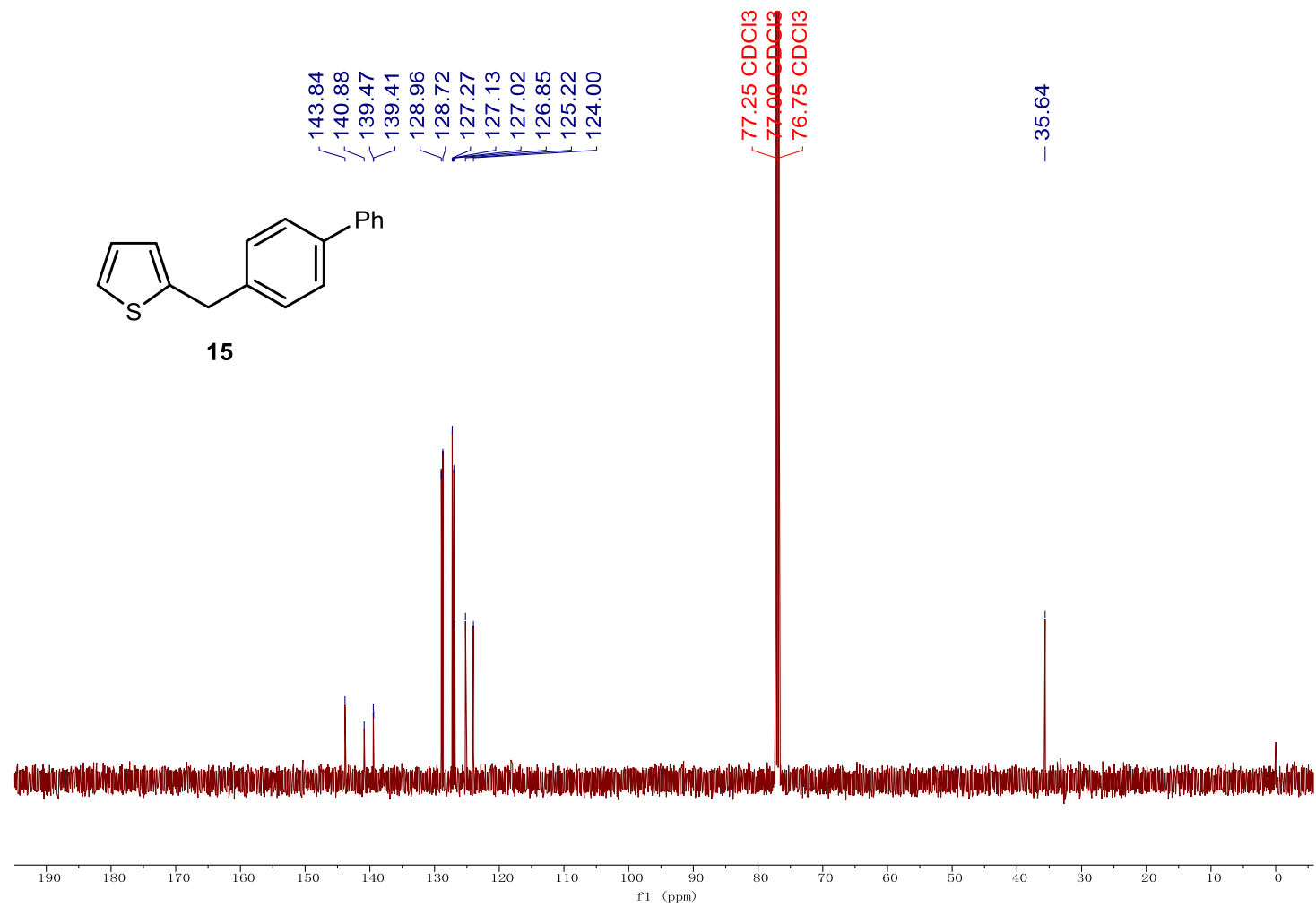
${ }^{1} \mathrm{H}$ NMR $500 \mathrm{MHz}, \mathrm{CDCl}_{3}$

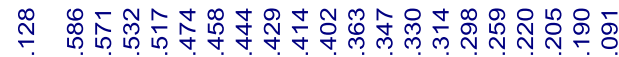

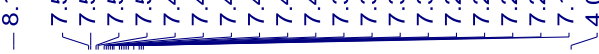

$\underset{\substack{i \\ \leftarrow}}{i}$<smiles>CC(C)(C)OC(=O)n1cc(Cc2ccc(-c3ccccc3)cc2)c2ccccc21</smiles>

16

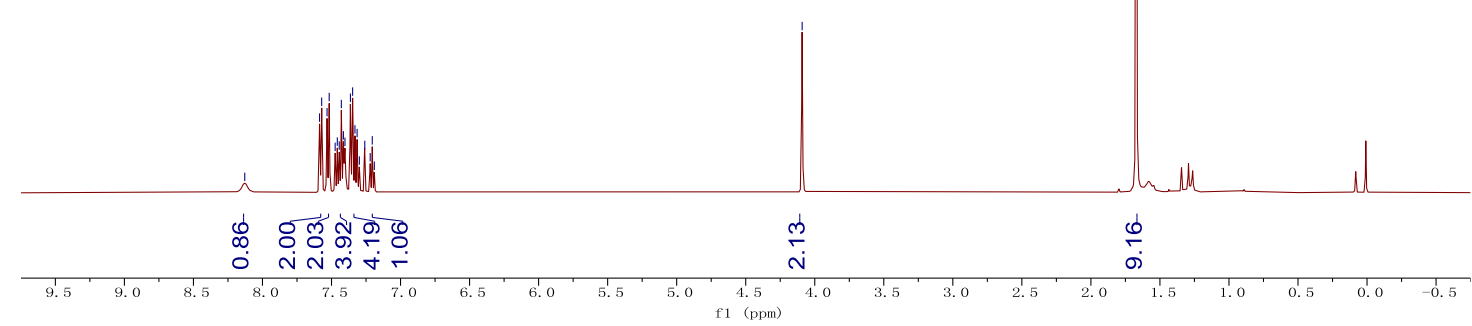

${ }^{13} \mathrm{C}$ NMR $125 \mathrm{MHz}, \mathrm{CDCl}_{3}$
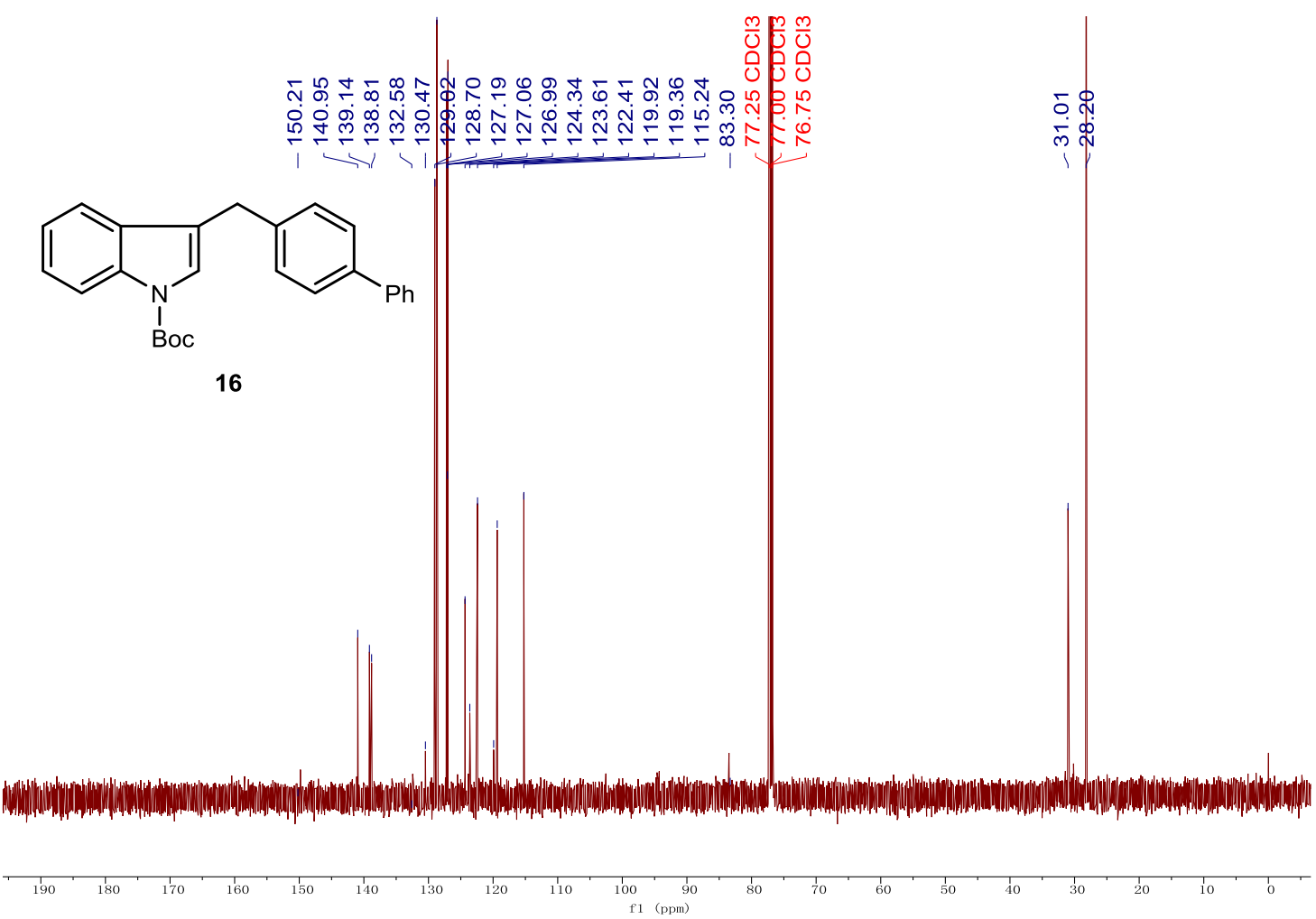
${ }^{1} \mathrm{H}$ NMR $500 \mathrm{MHz}, \mathrm{CDCl}_{3}$

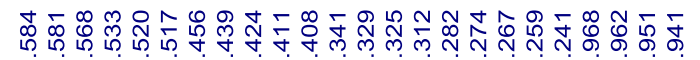

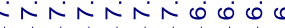

Nิ
ホ
I

$m$
0
0
0<smiles>c1ccc(-c2ccc(Cc3ccsc3)cc2)cc1</smiles>

17

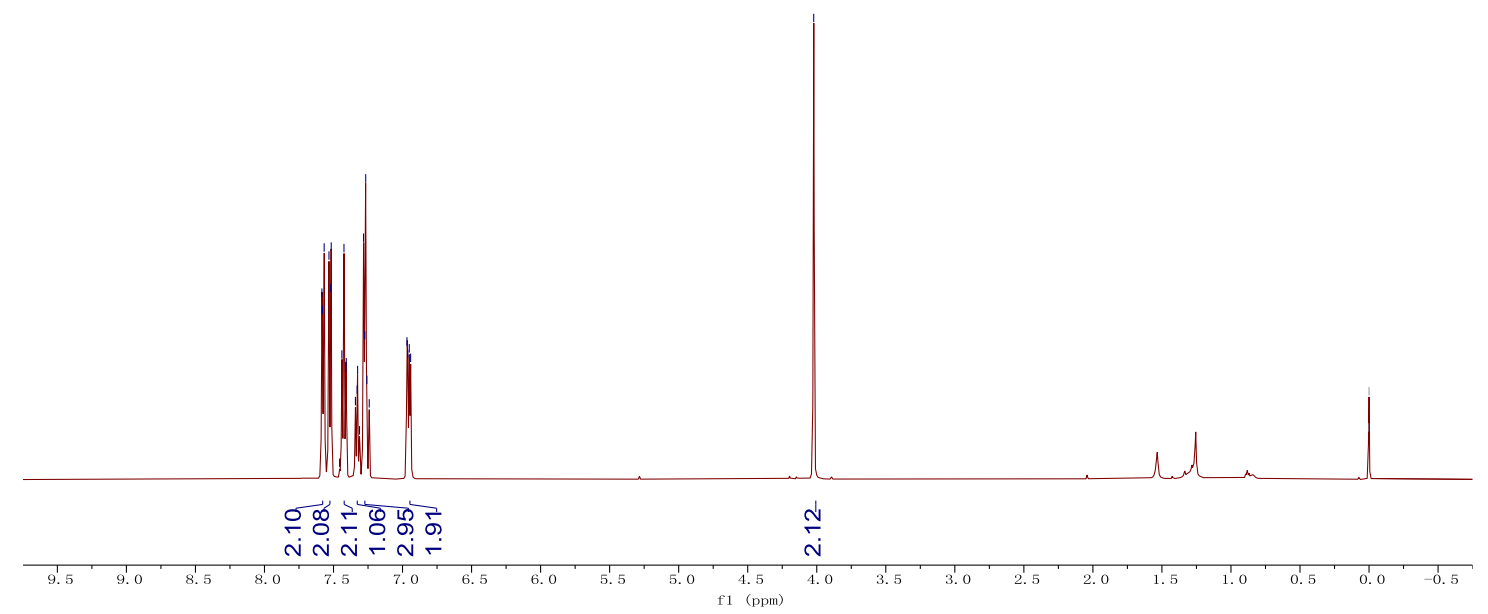

${ }^{13} \mathrm{C}$ NMR $125 \mathrm{MHz}, \mathrm{CDCl}_{3}$
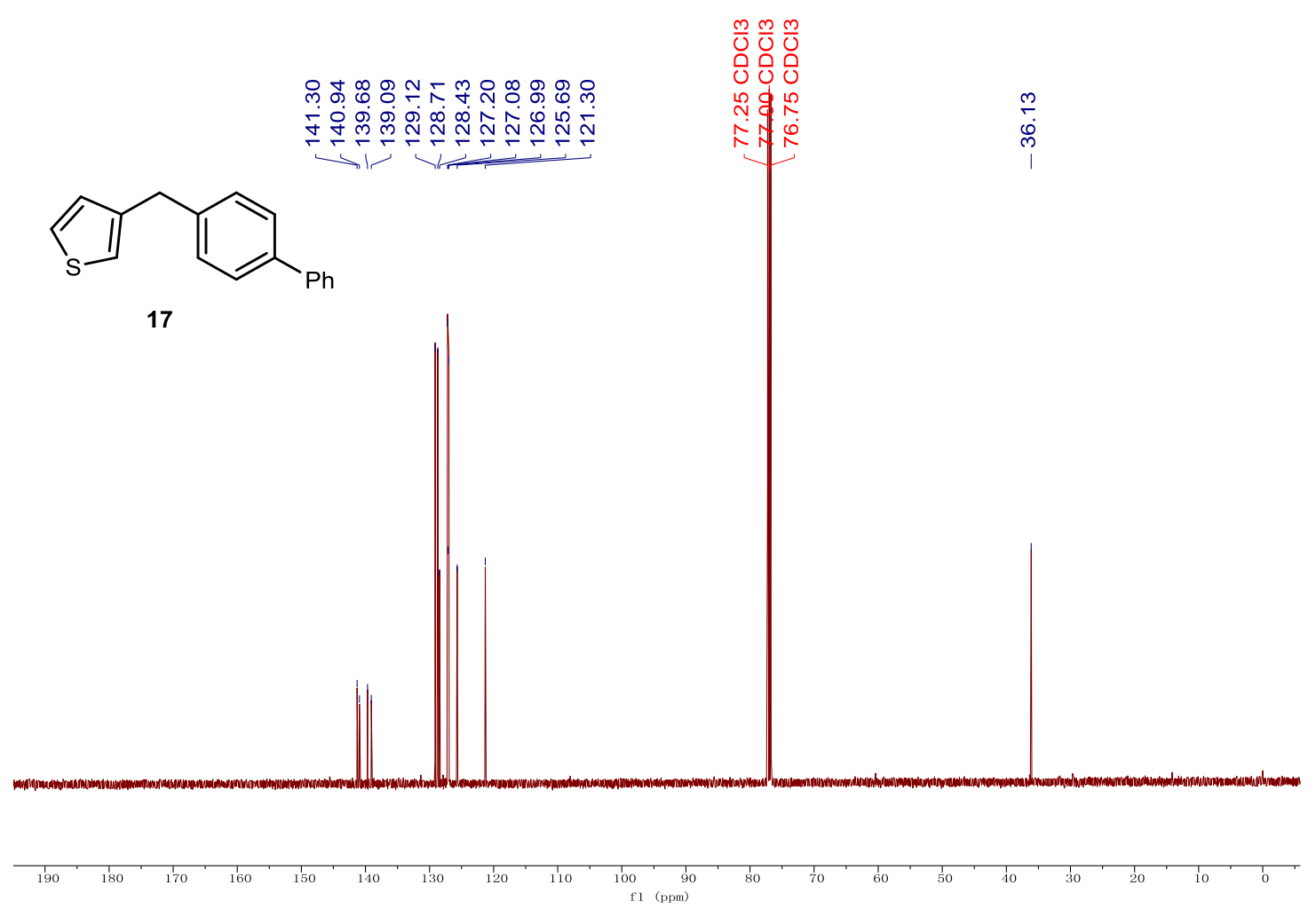
${ }^{1} \mathrm{H}$ NMR $500 \mathrm{MHz}, \mathrm{CDCl}_{3}$

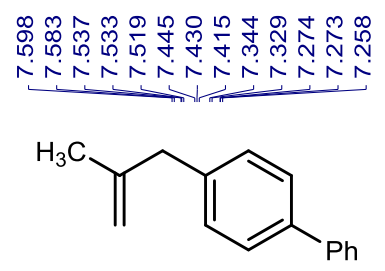

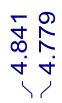

¿্口.

$\stackrel{ }{\grave{1}}$

18

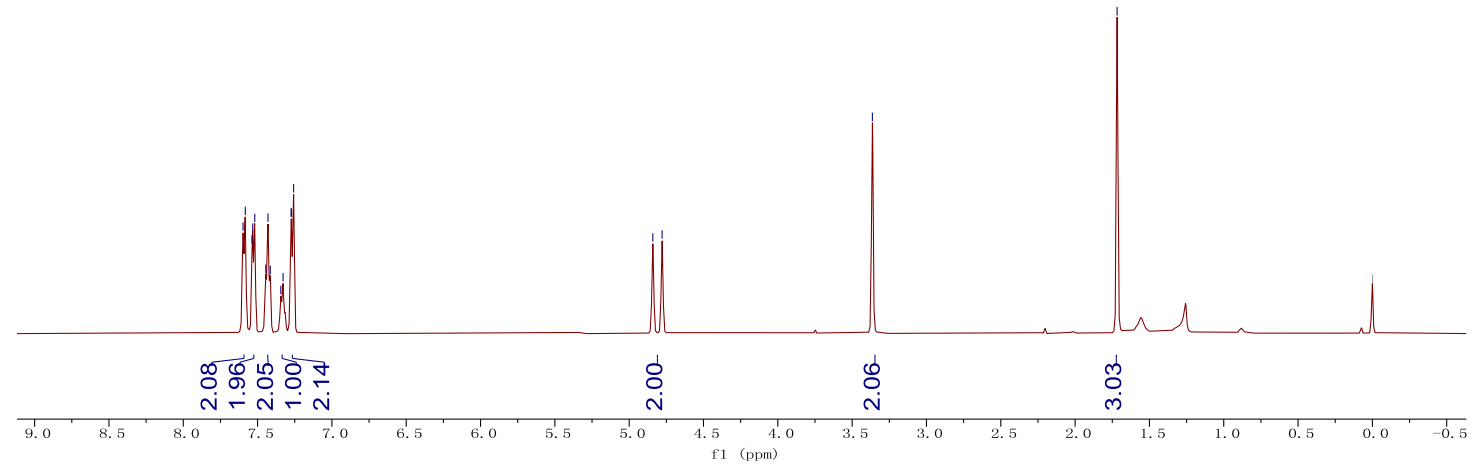

${ }^{13} \mathrm{C}$ NMR $125 \mathrm{MHz}, \mathrm{CDCl}_{3}$
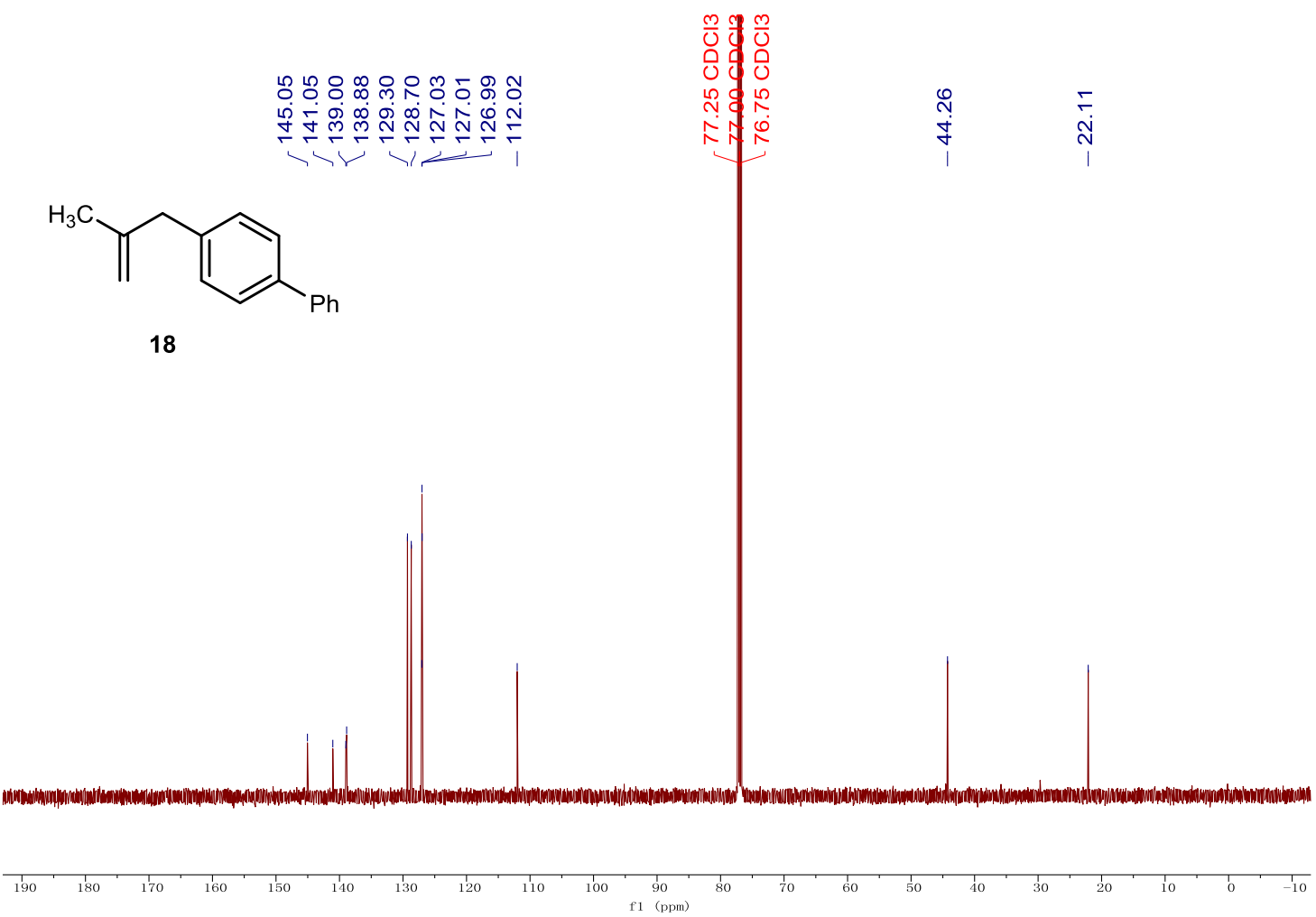
${ }^{1} \mathrm{H}$ NMR $500 \mathrm{MHz}, \mathrm{CDCl}_{3}$

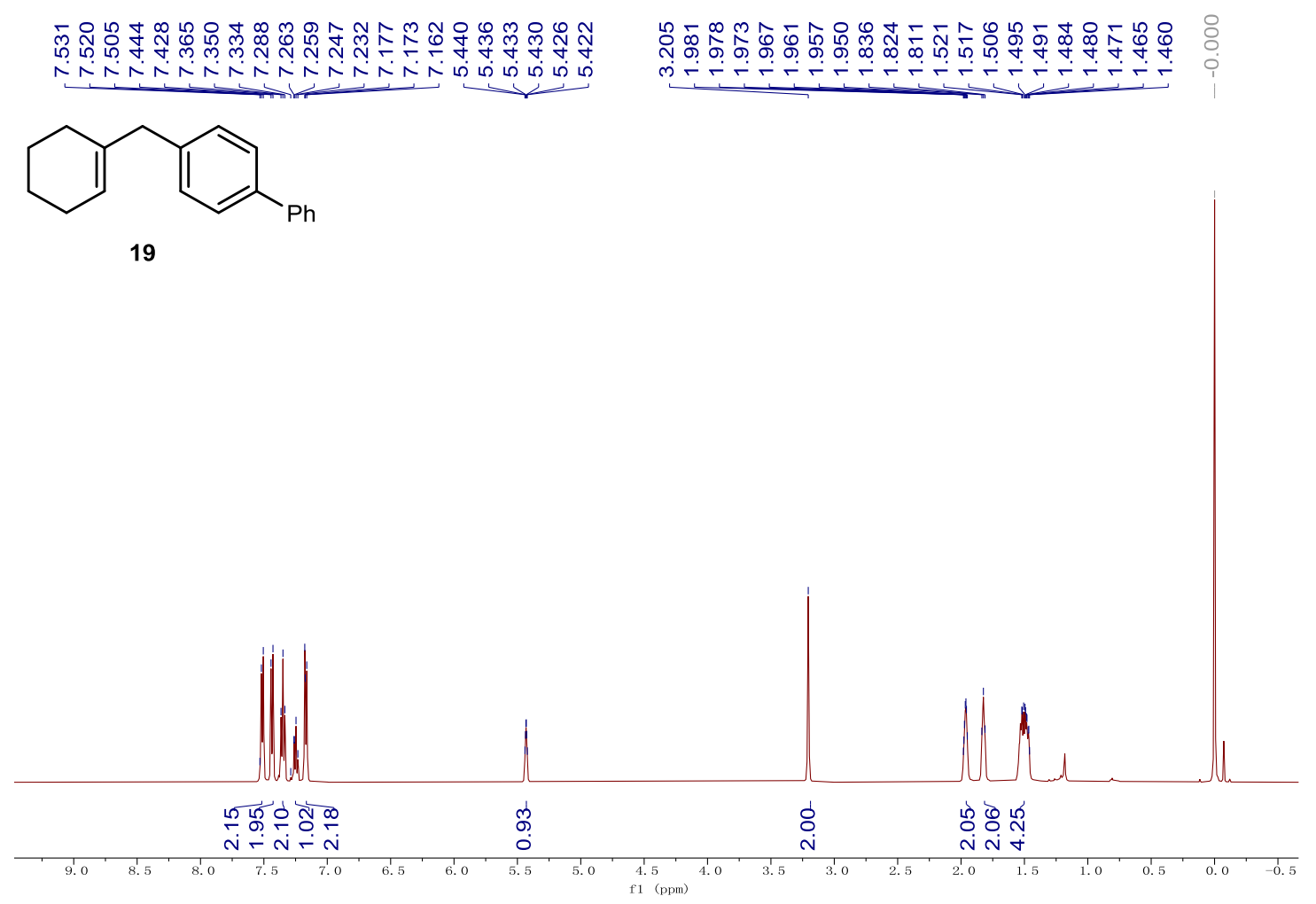

${ }^{13} \mathrm{C}$ NMR $100 \mathrm{MHz}, \mathrm{CDCl}_{3}$
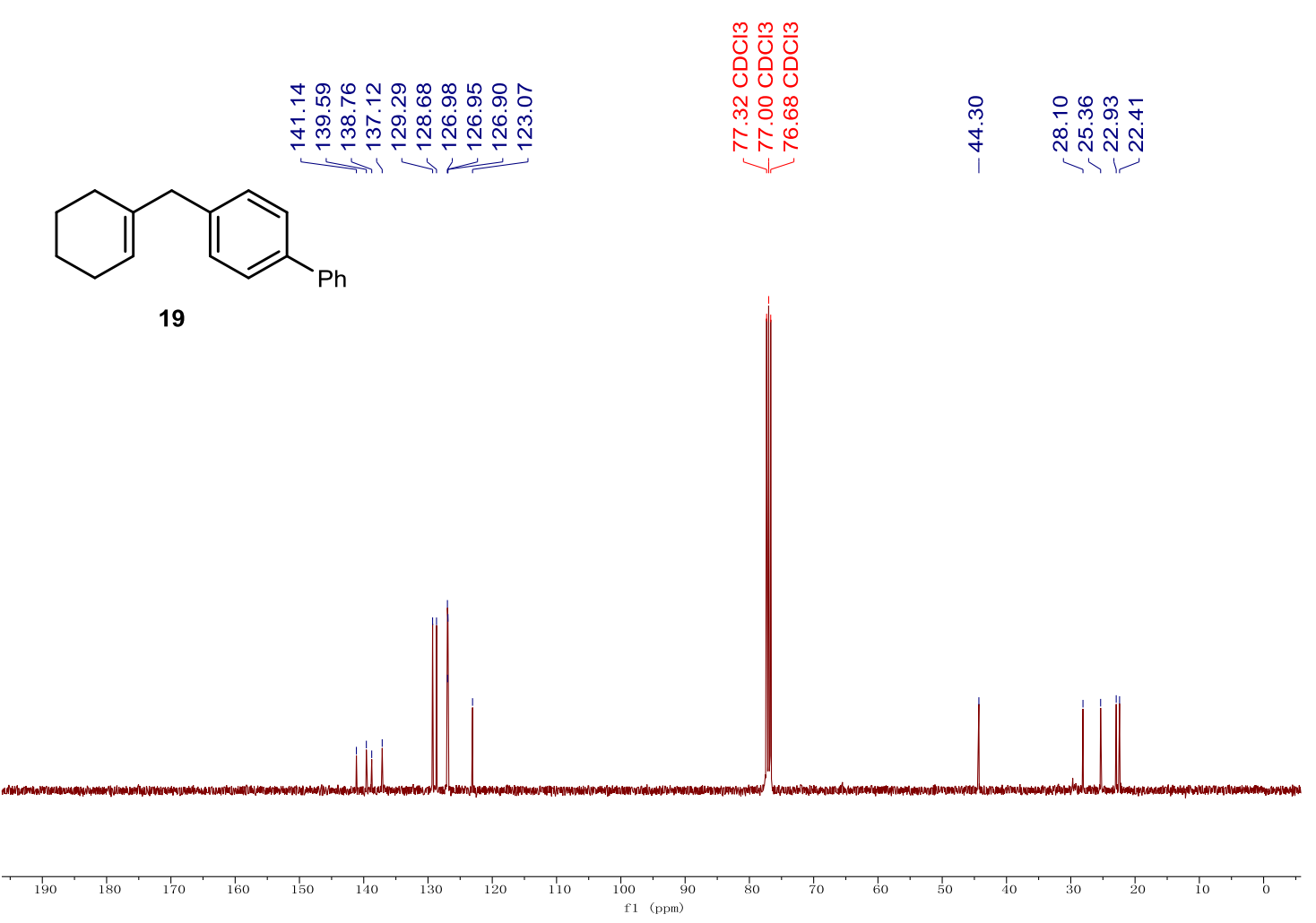
${ }^{1} \mathrm{H}$ NMR $500 \mathrm{MHz}, \mathrm{CDCl}_{3}$

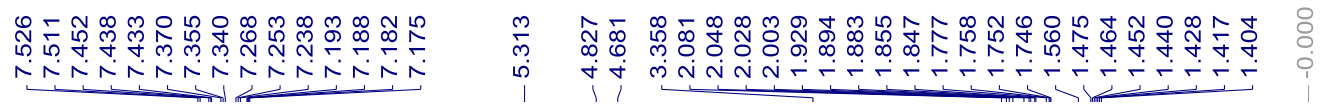<smiles>C=C(Cc1ccc(-c2ccccc2)cc1)C1CC=C(C)CC1</smiles>

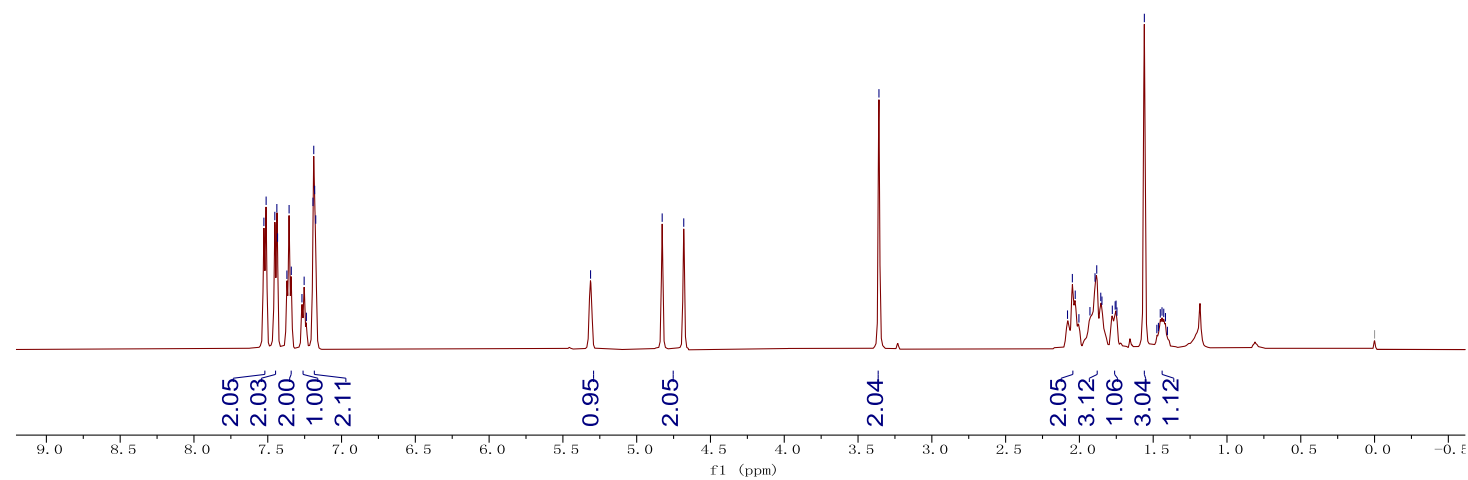

${ }^{13} \mathrm{C}$ NMR $125 \mathrm{MHz}, \mathrm{CDCl}_{3}$

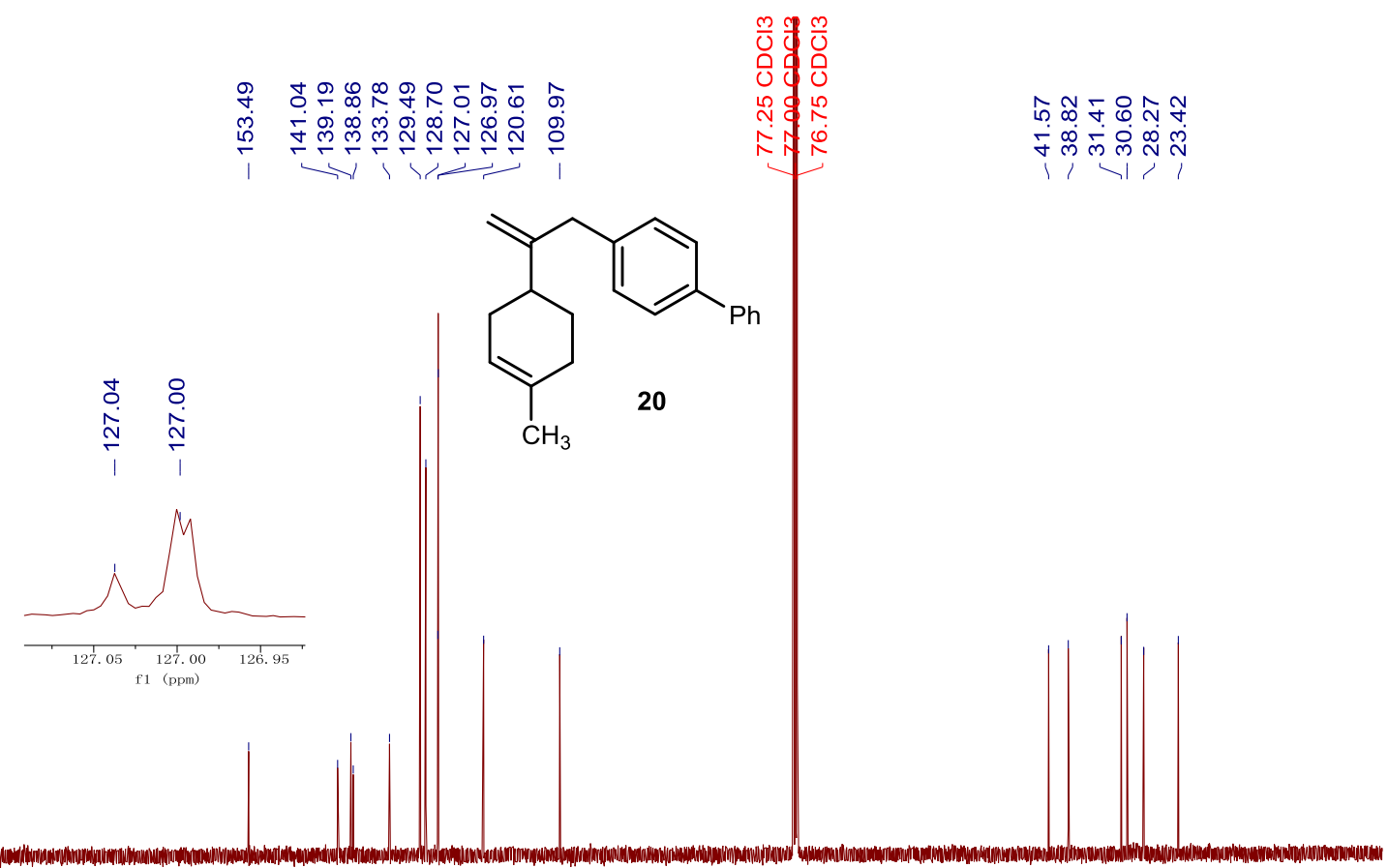

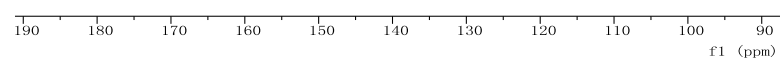


${ }^{1} \mathrm{H}$ NMR $500 \mathrm{MHz}, \mathrm{CDCl}_{3}$

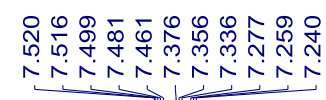

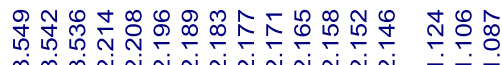

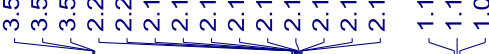

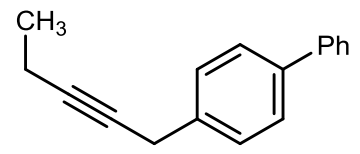

21

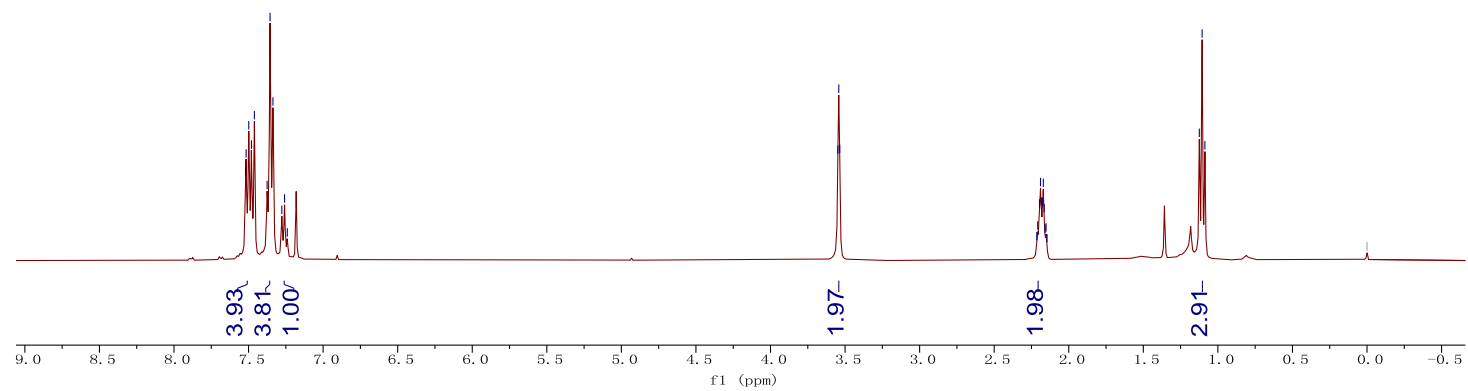

${ }^{13} \mathrm{C} \mathrm{NMR} 125 \mathrm{MHz}, \mathrm{CDCl}_{3}$

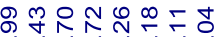

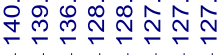

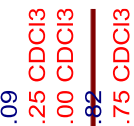

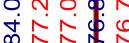

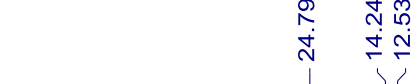

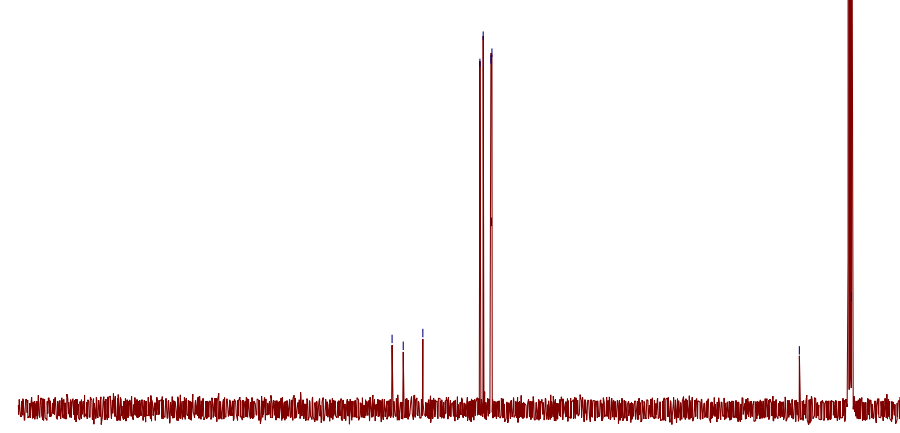

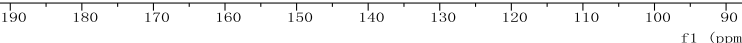


${ }^{1} \mathrm{H}$ NMR $500 \mathrm{MHz}, \mathrm{CDCl}_{3}$
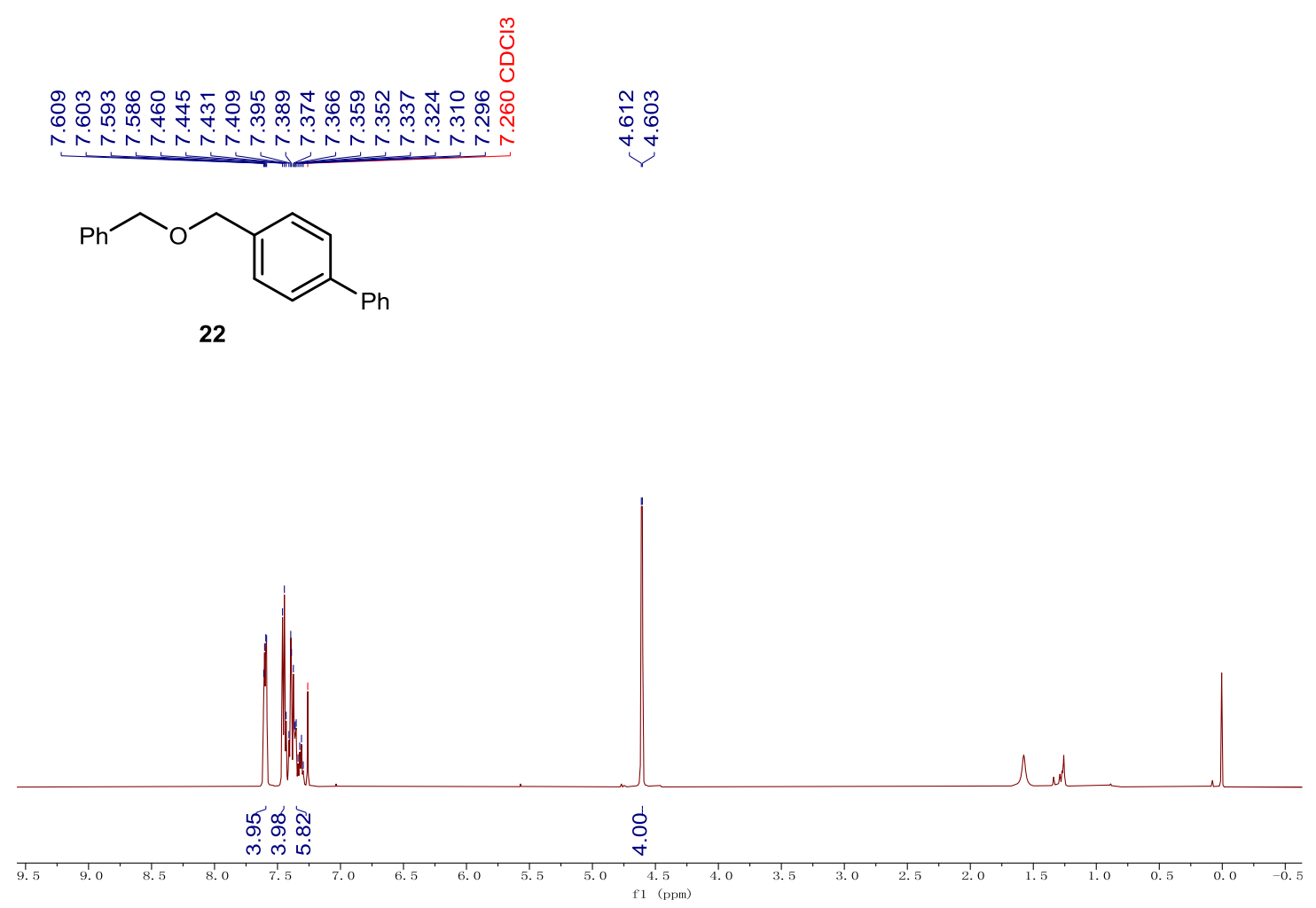

${ }^{13} \mathrm{C}$ NMR $125 \mathrm{MHz}, \mathrm{CDCl}_{3}$ 

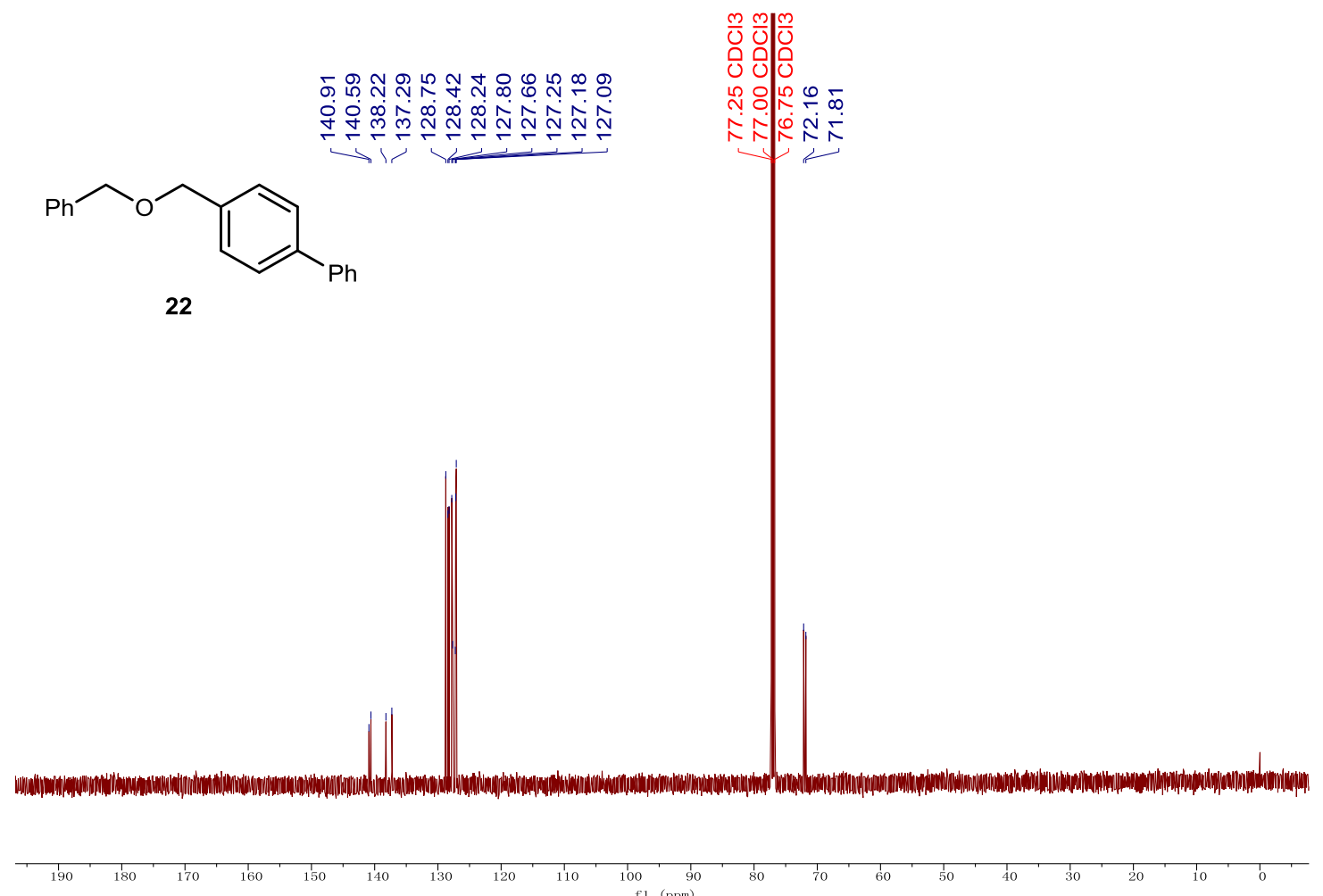

${ }^{1} \mathrm{H}$ NMR $500 \mathrm{MHz}, \mathrm{CDCl}_{3}$

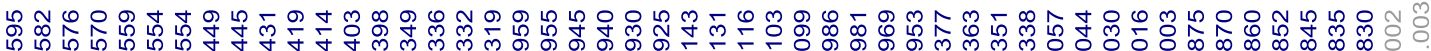

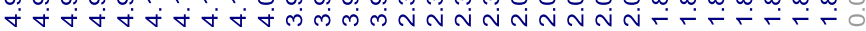<smiles>c1ccc(-c2ccc(C3CCCO3)cc2)cc1</smiles>

23

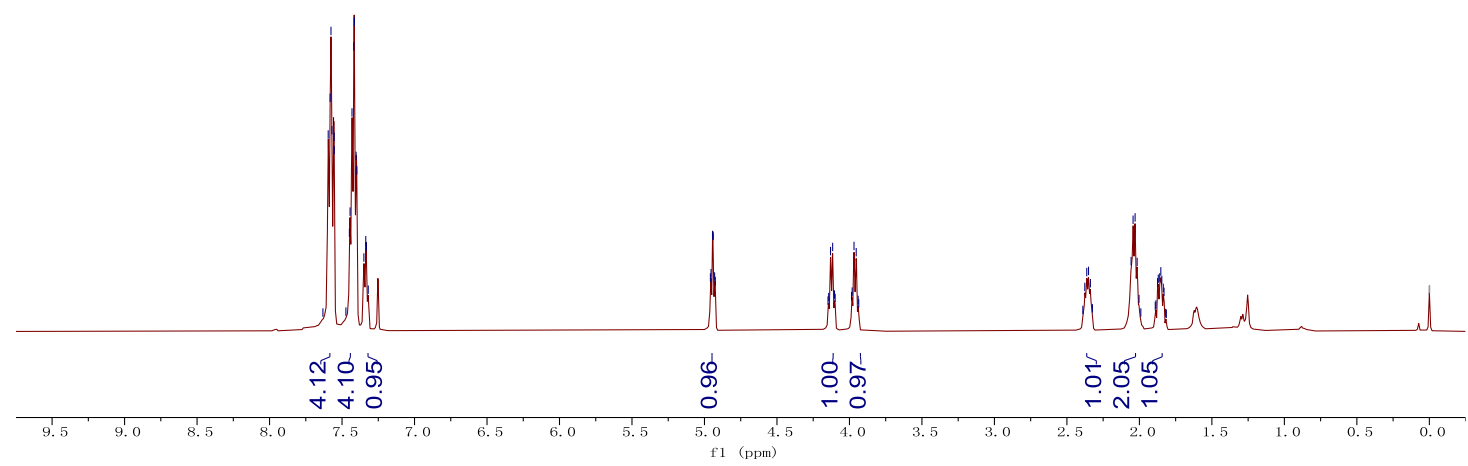

${ }^{13} \mathrm{C}$ NMR $125 \mathrm{MHz}, \mathrm{CDCl}_{3}$ 

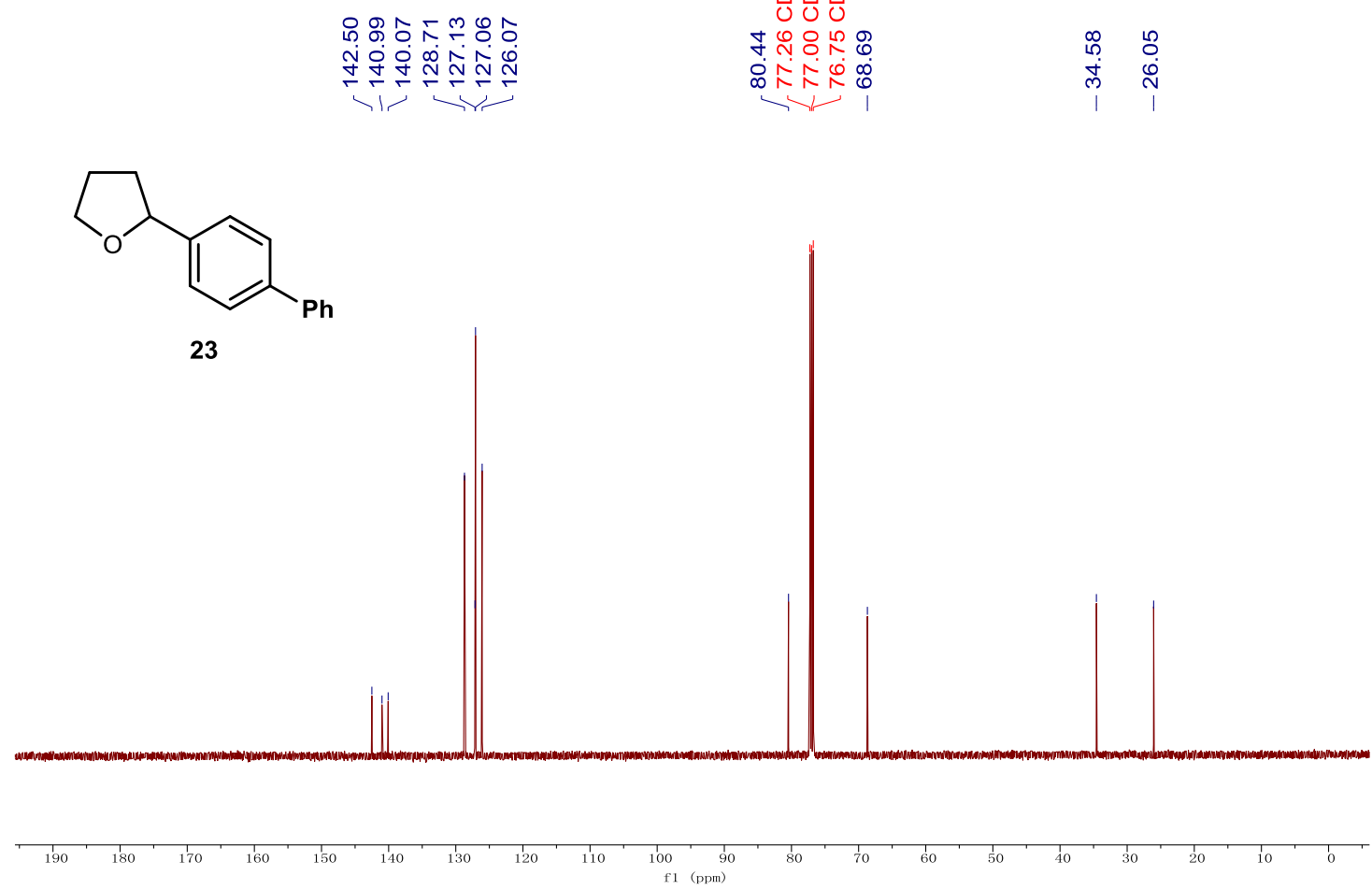

${ }^{1} \mathrm{H}$ NMR $500 \mathrm{MHz}, \mathrm{CDCl}_{3}$

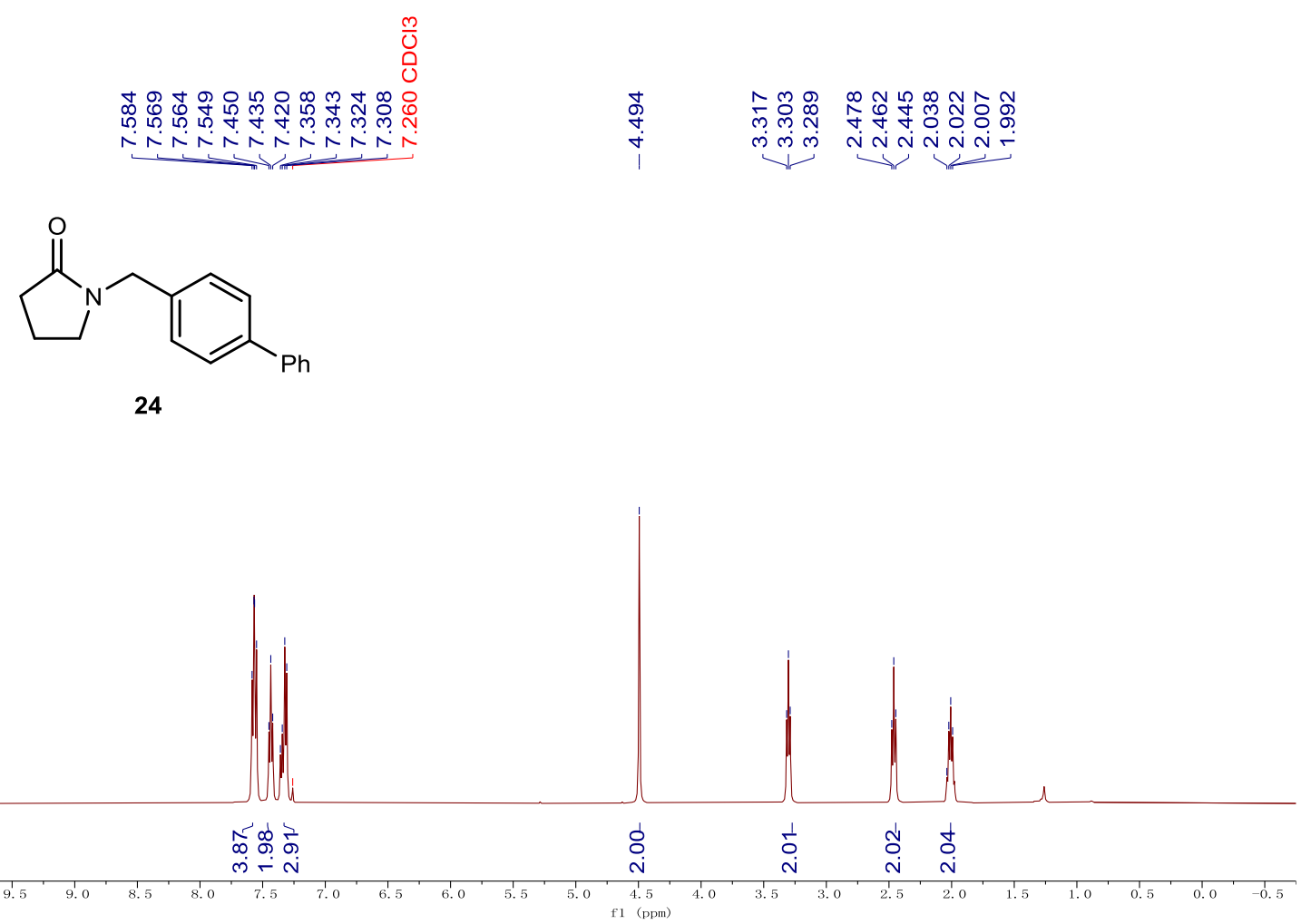

${ }^{13} \mathrm{C}$ NMR $125 \mathrm{MHz}, \mathrm{CDCl}_{3}$ 


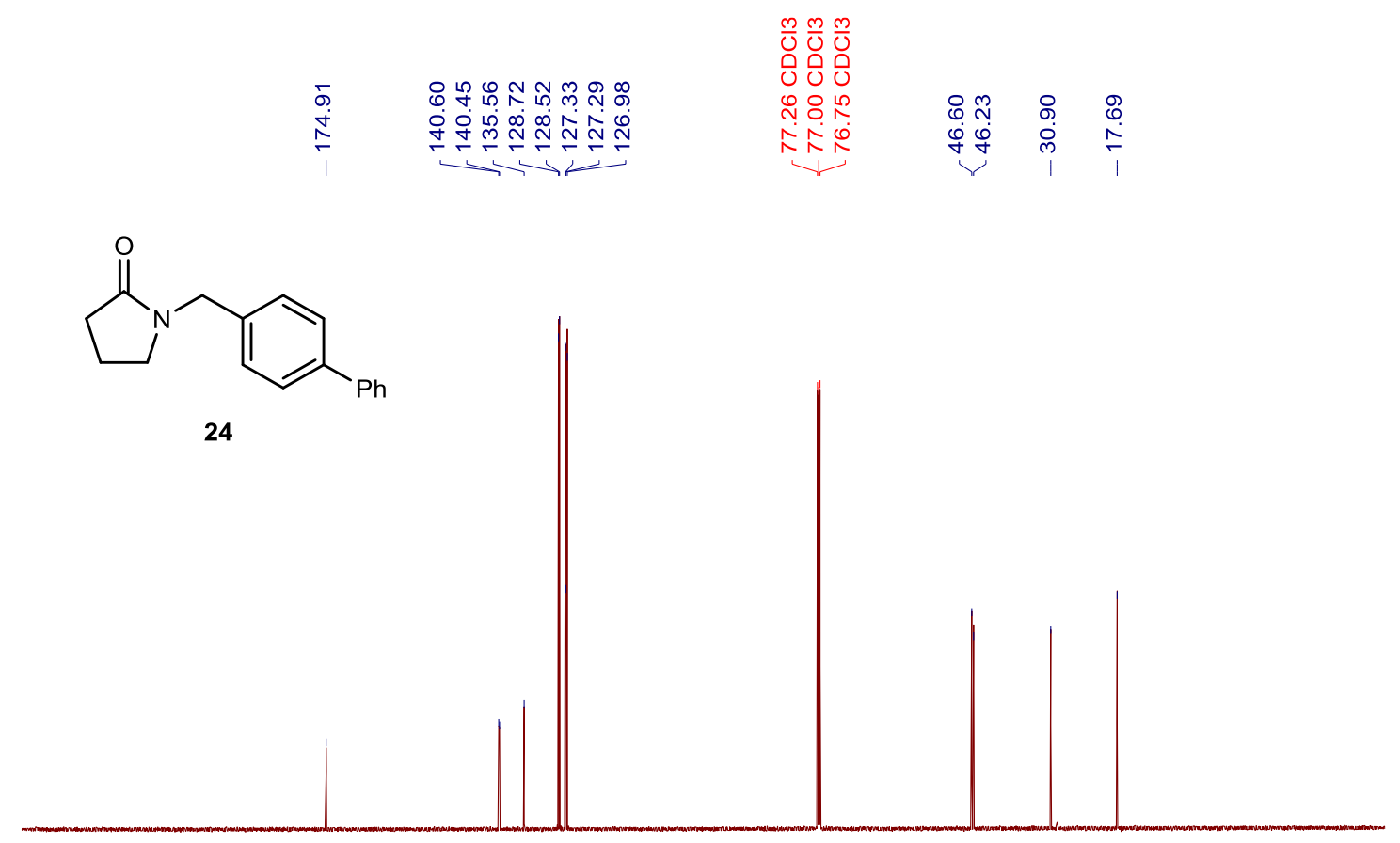

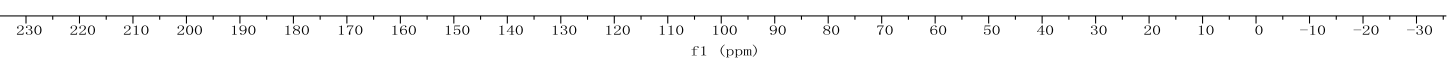

${ }^{1} \mathrm{H}$ NMR $500 \mathrm{MHz}, \mathrm{CDCl}_{3}$

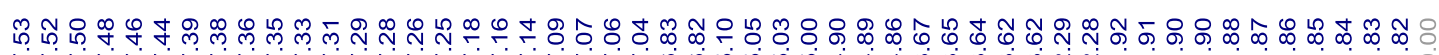

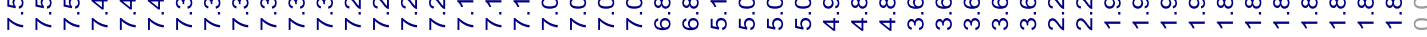

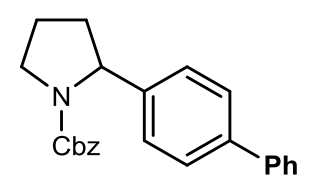

25

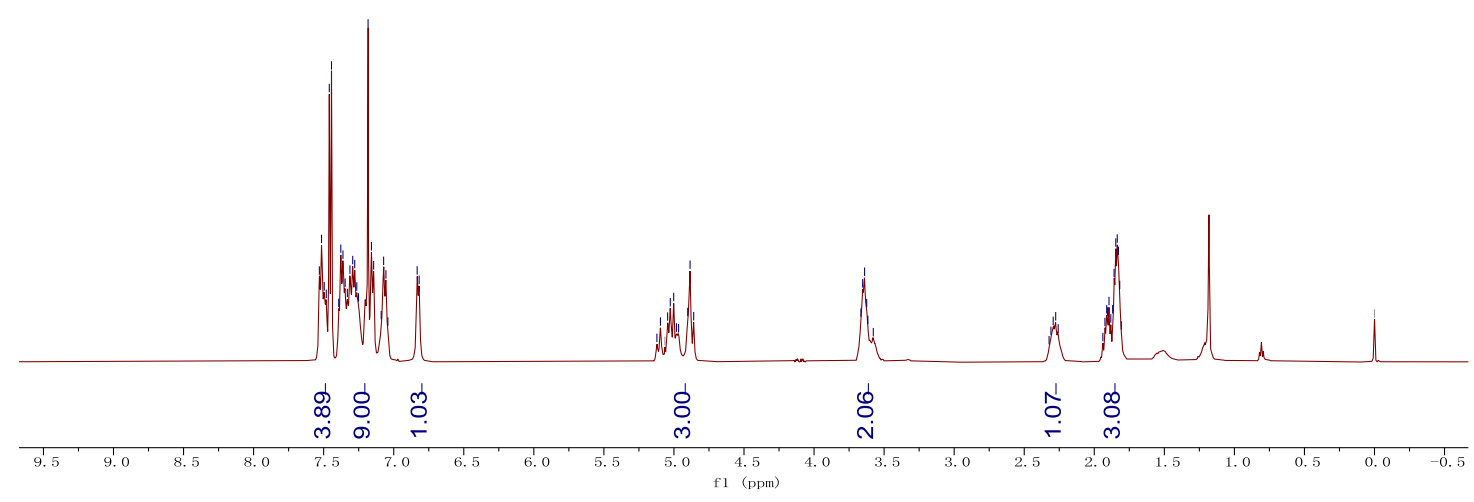

${ }^{13} \mathrm{C} \mathrm{NMR} 125 \mathrm{MHz}, \mathrm{CDCl}_{3}$ 

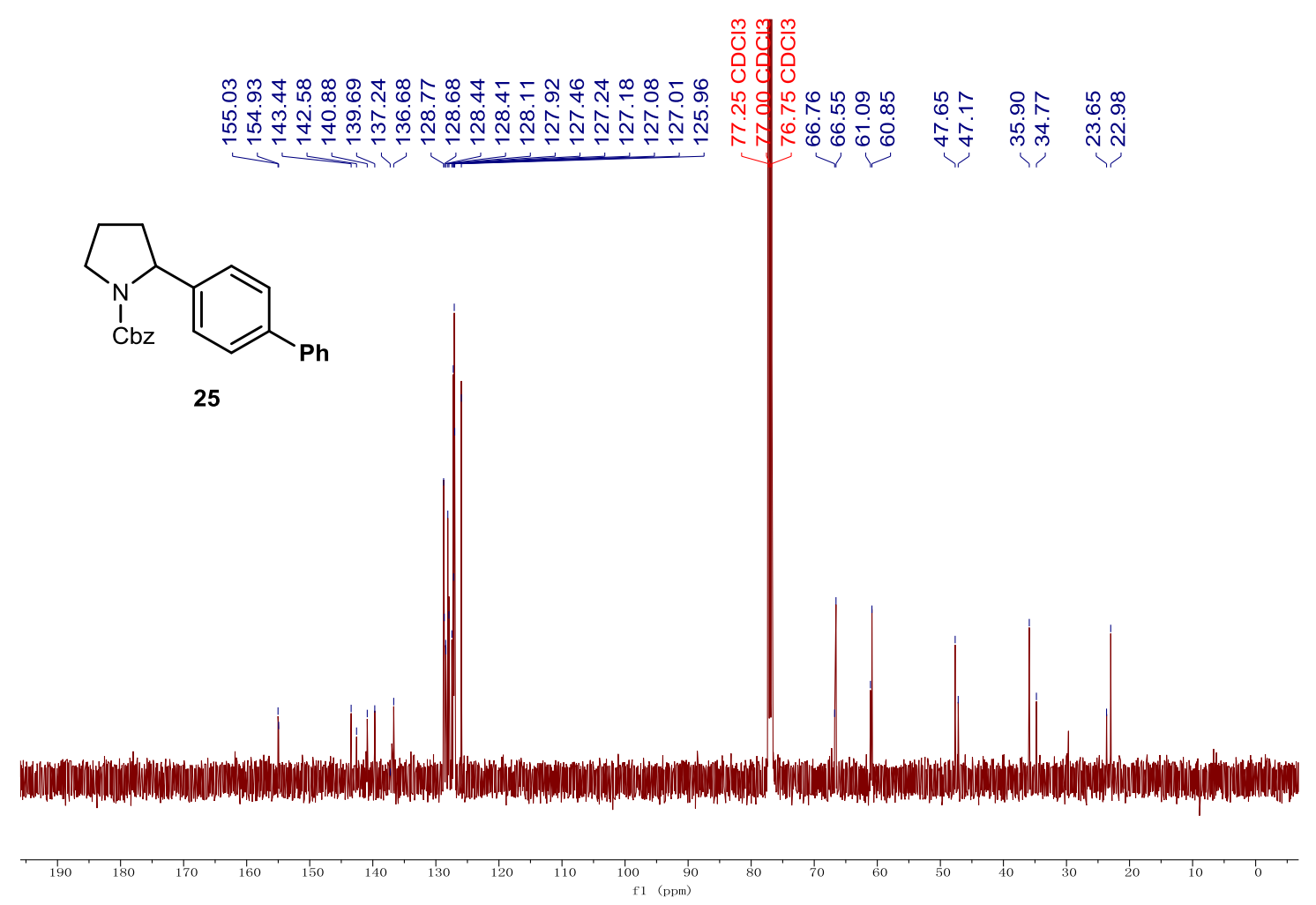

${ }^{13} \mathrm{C}$ NMR $125 \mathrm{MHz},\left(\mathrm{CD}_{3}\right)_{2} \mathrm{CO}$

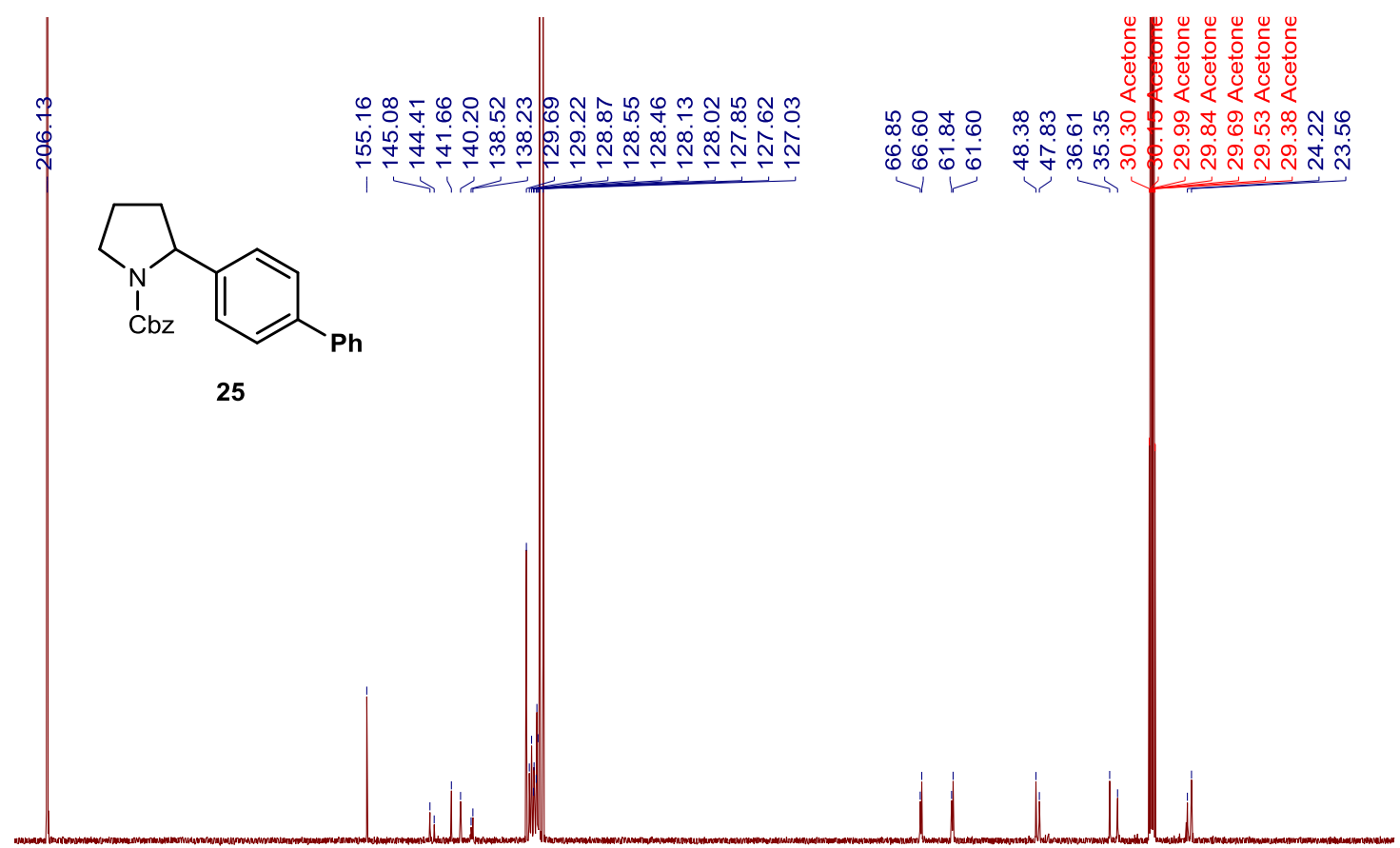

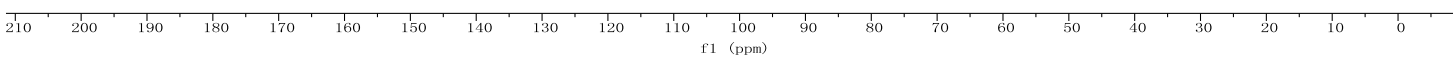

${ }^{1} \mathrm{H}$ NMR $500 \mathrm{MHz}, \mathrm{CDCl}_{3}$ 
$\frac{m}{\mathrm{O}}$

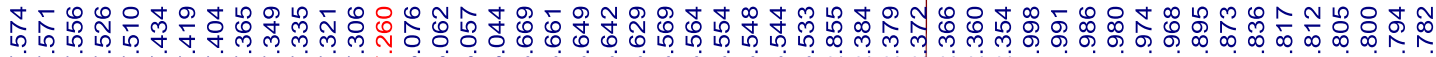

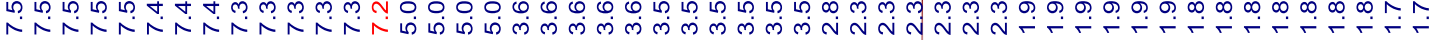

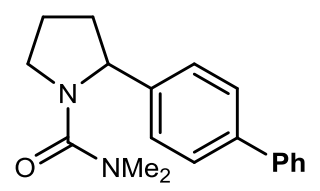

26

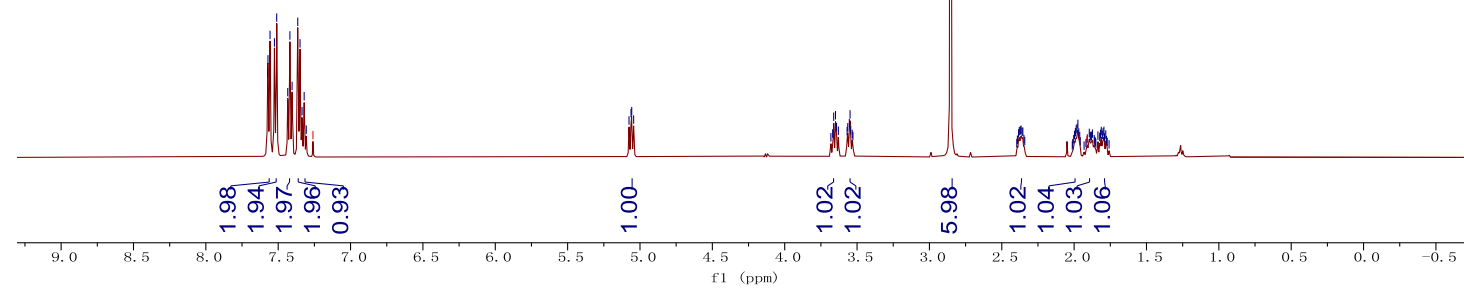

${ }^{13} \mathrm{C} \mathrm{NMR} 125 \mathrm{MHz}, \mathrm{CDCl}_{3}$

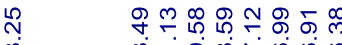

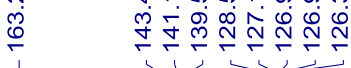

$\frac{m}{0} \frac{m}{0} \frac{m}{0}$

잉

㝊造<smiles>CNC(=O)N1CCCC1c1ccc(-c2ccccc2)cc1</smiles>

26

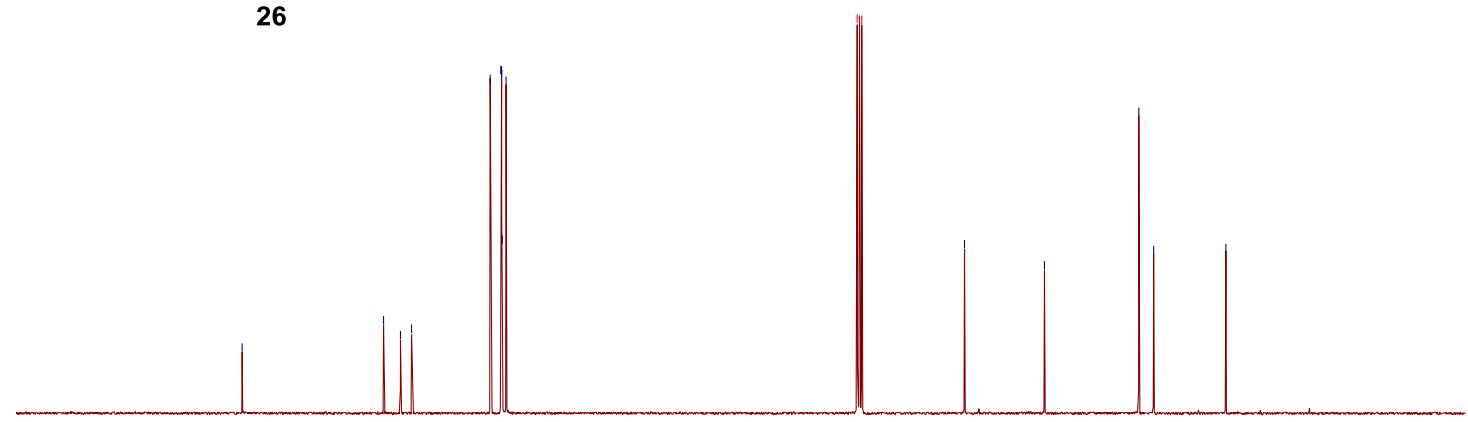


${ }^{13} \mathrm{C}$ NMR $125 \mathrm{MHz},\left(\mathrm{CD}_{3}\right)_{2} \mathrm{CO}$
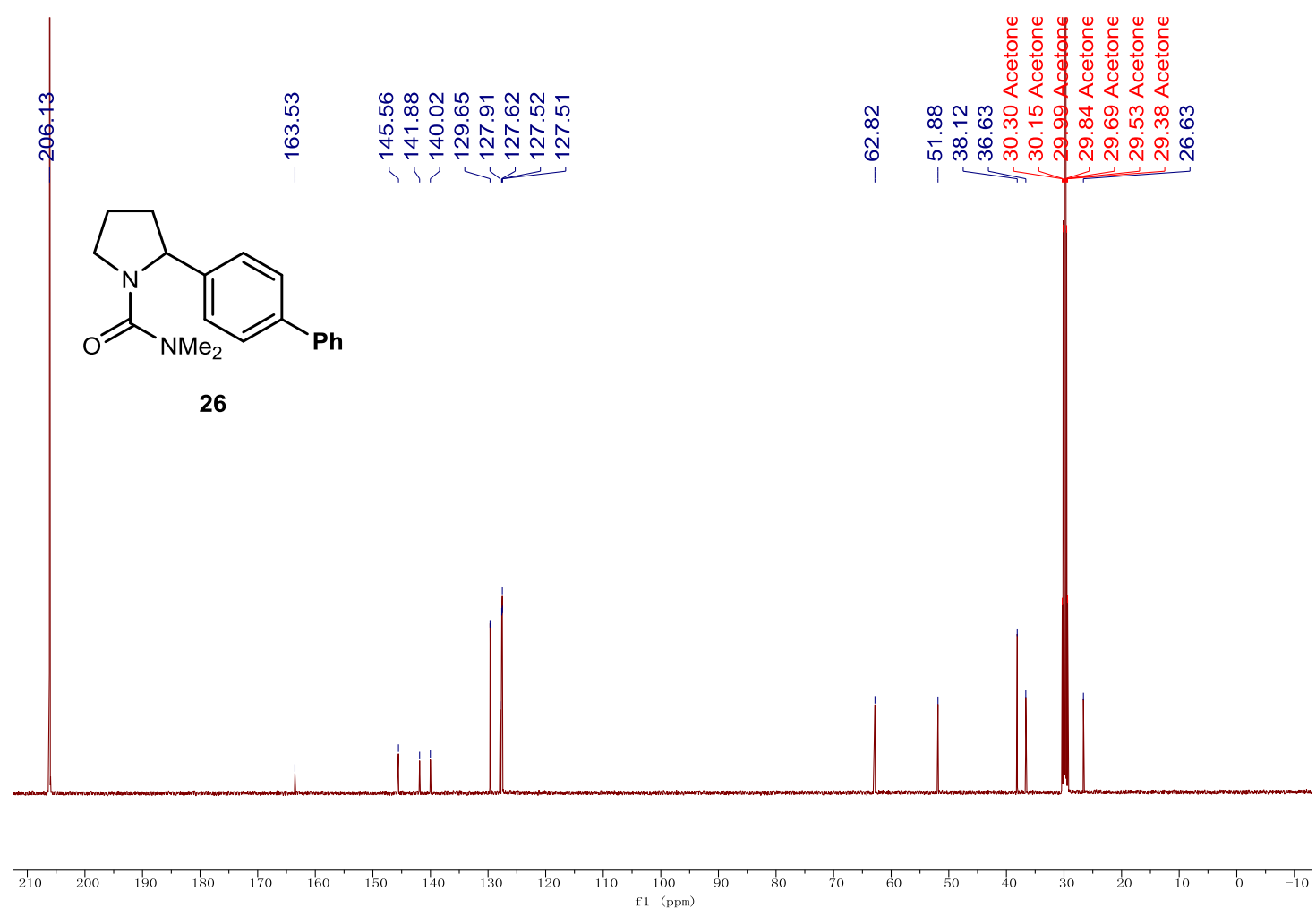

${ }^{1} \mathrm{H}$ NMR $500 \mathrm{MHz}, \mathrm{CDCl}_{3}$

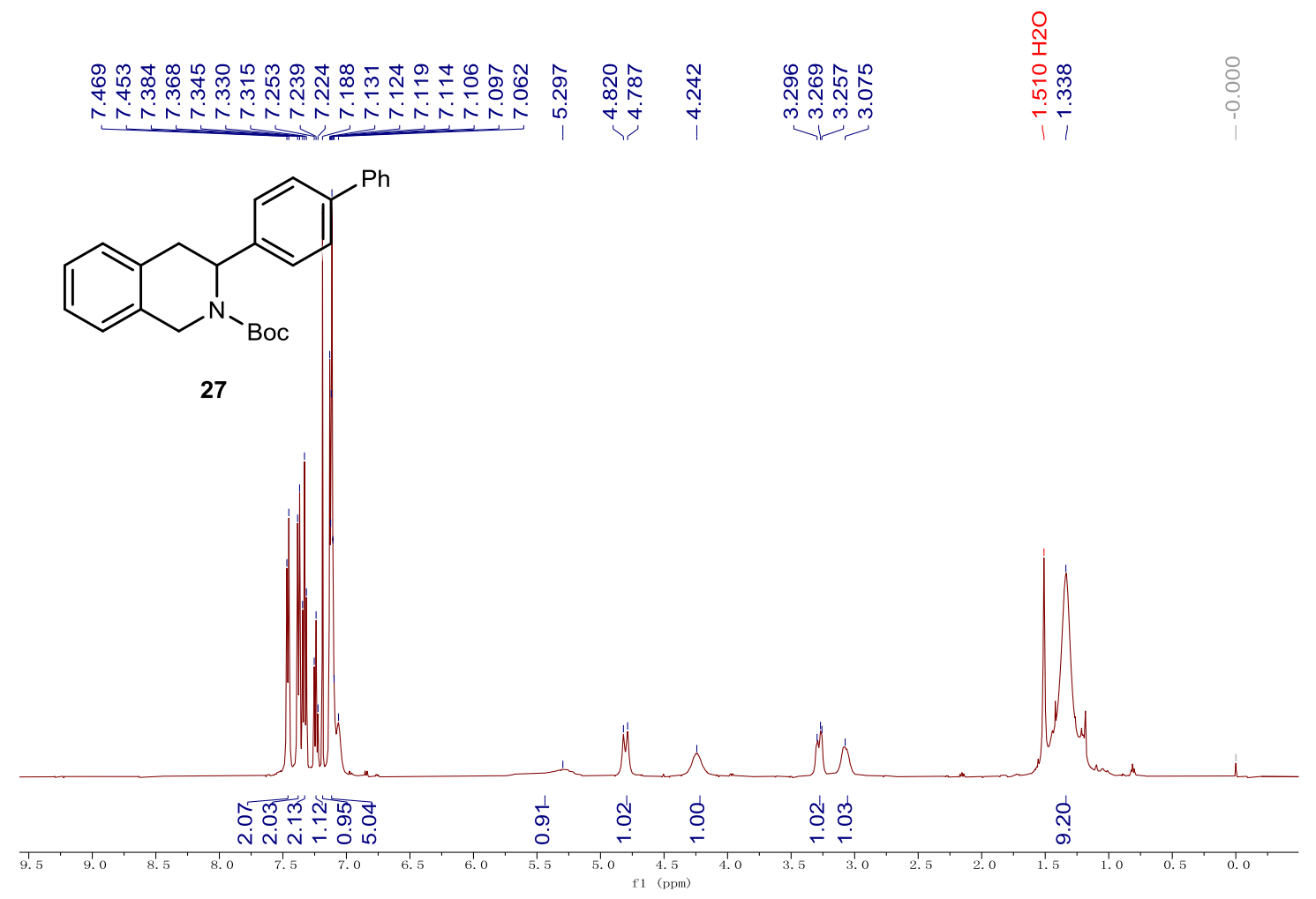


${ }^{13} \mathrm{C}$ NMR $125 \mathrm{MHz}, \mathrm{CDCl}_{3}$
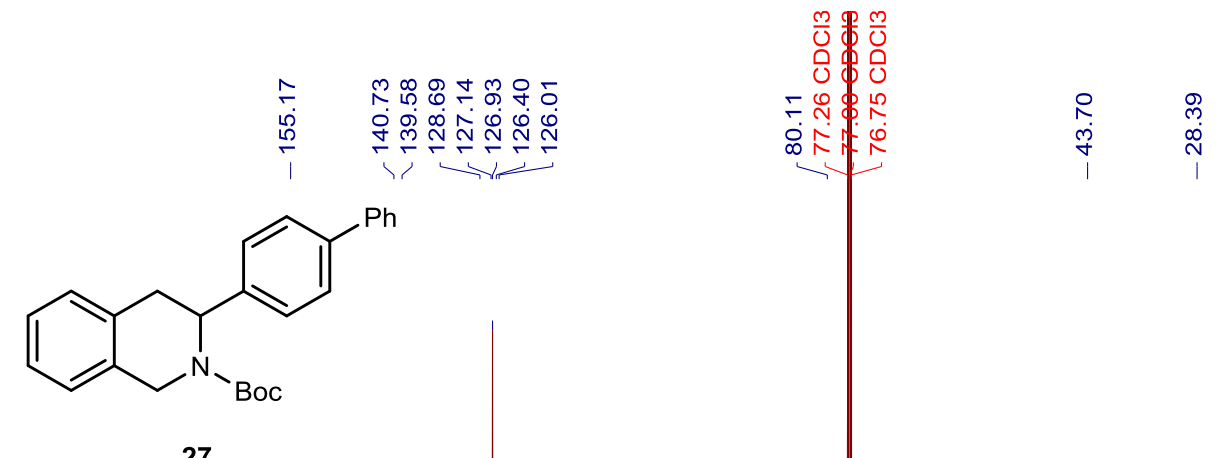

27

${ }^{13} \mathrm{C}$ NMR $125 \mathrm{MHz},\left(\mathrm{CD}_{3}\right)_{2} \mathrm{CO}$

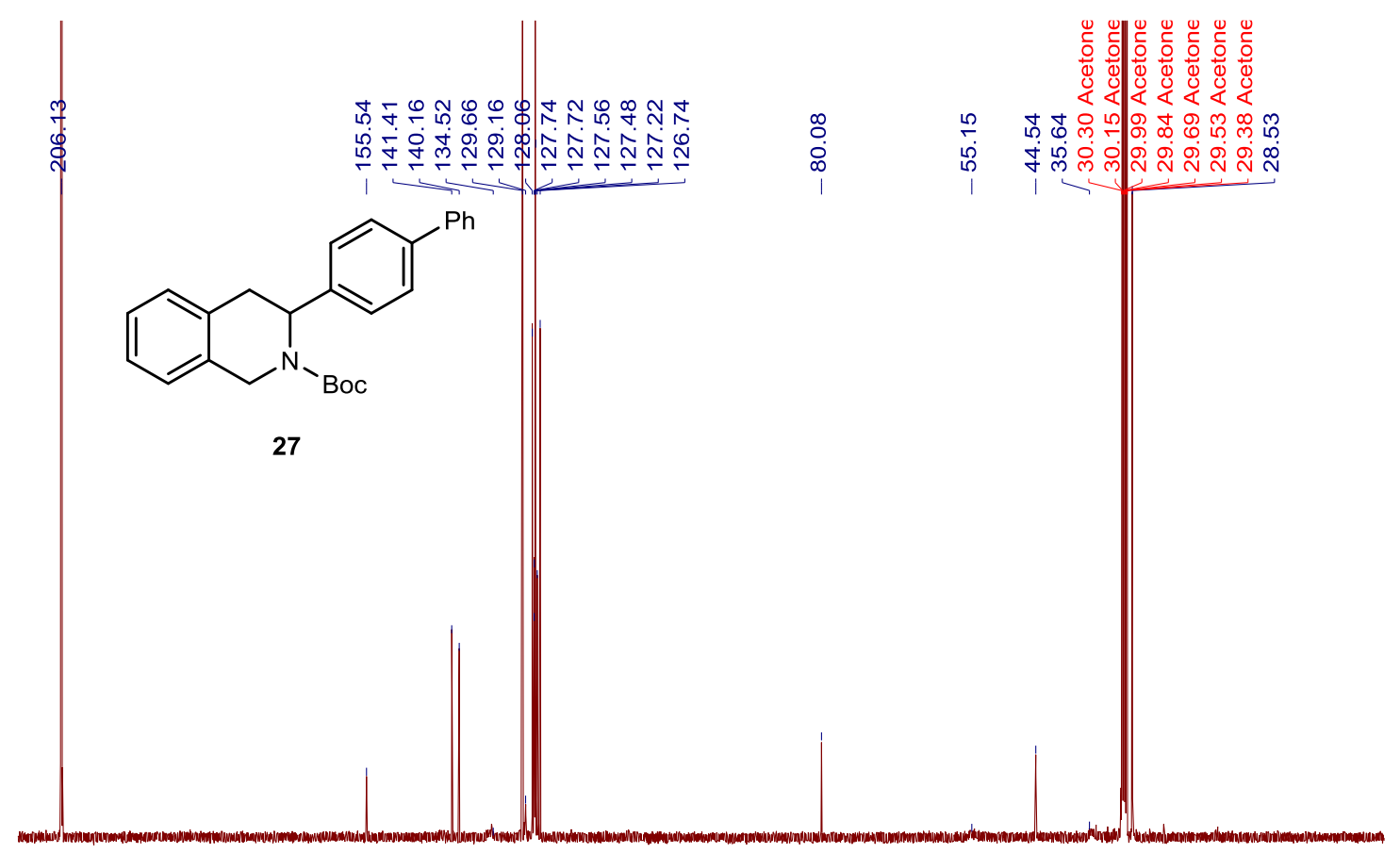

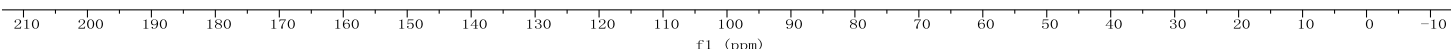


${ }^{1} \mathrm{H}$ NMR $400 \mathrm{MHz}, \mathrm{CDCl}_{3}$

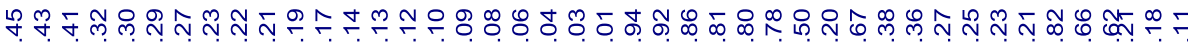

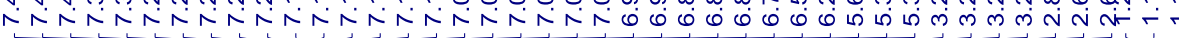<smiles>O=C(c1ccccc1)N1C(c2ccc(-c3ccccc3)cc2)Cc2ccccc2C1c1ccccc1</smiles>

28

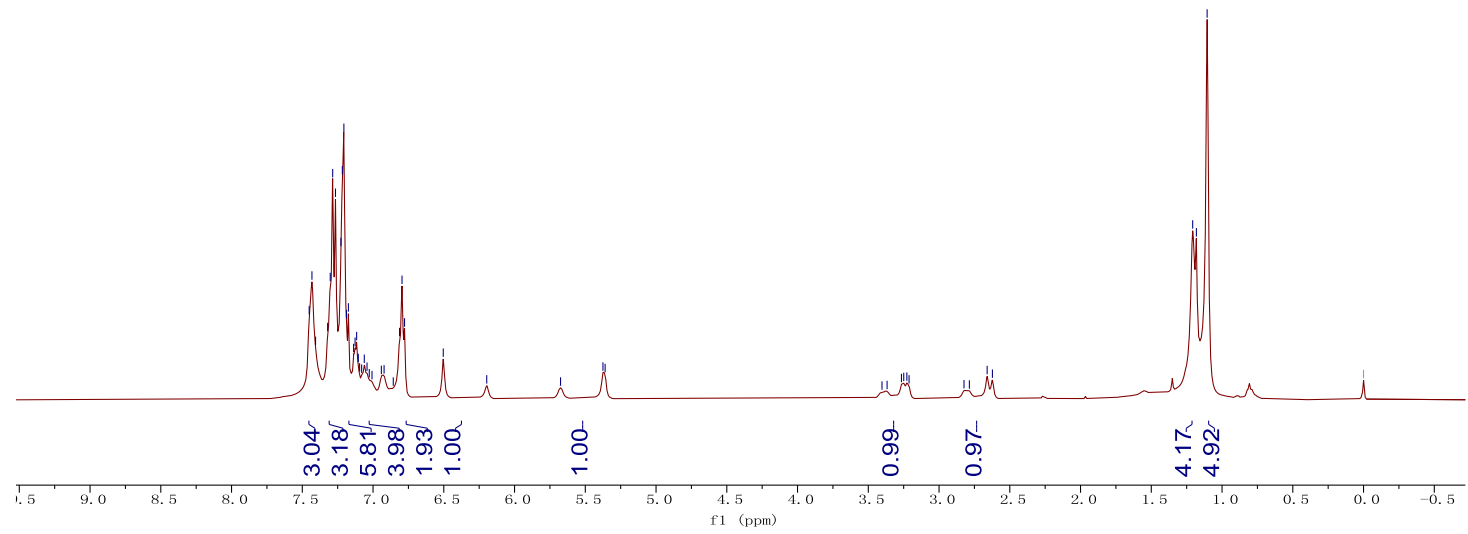

${ }^{13} \mathrm{C}$ NMR $100 \mathrm{MHz}, \mathrm{CDCl}_{3}$
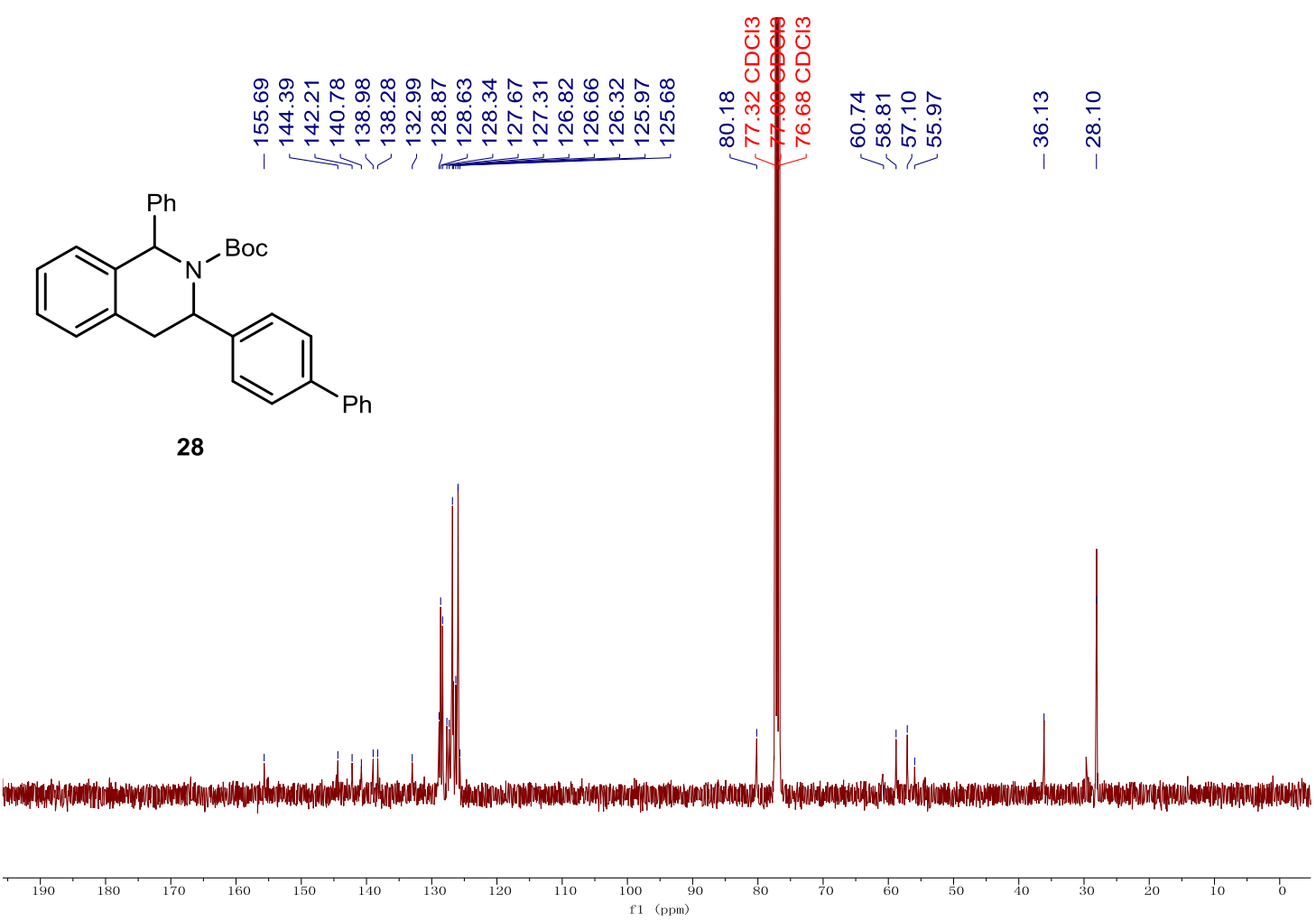
${ }^{13} \mathrm{C}$ NMR $100 \mathrm{MHz},\left(\mathrm{CD}_{3}\right)_{2} \mathrm{CO}$
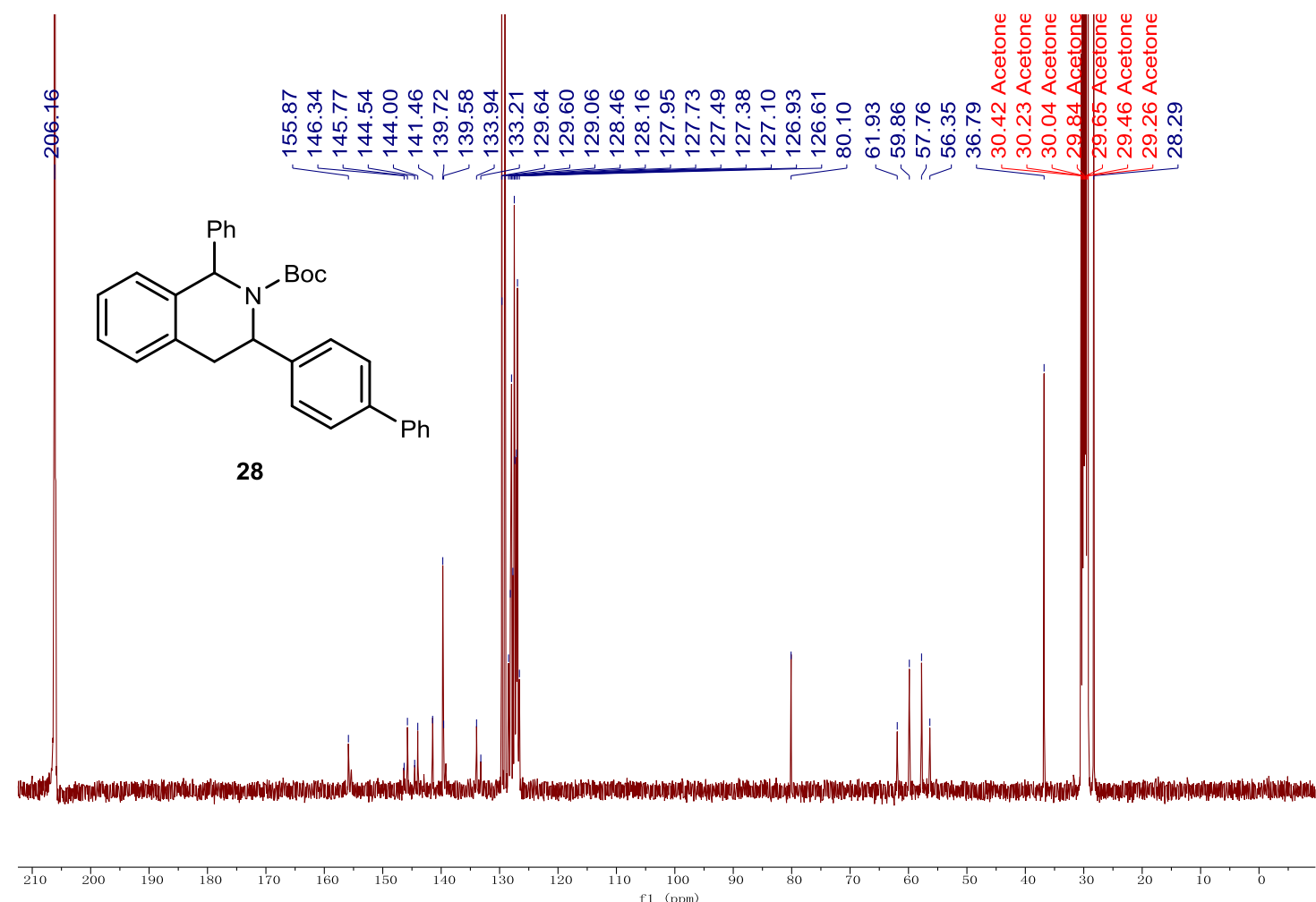

${ }^{1} \mathrm{H}$ NMR $500 \mathrm{MHz}, \mathrm{CDCl}_{3}$

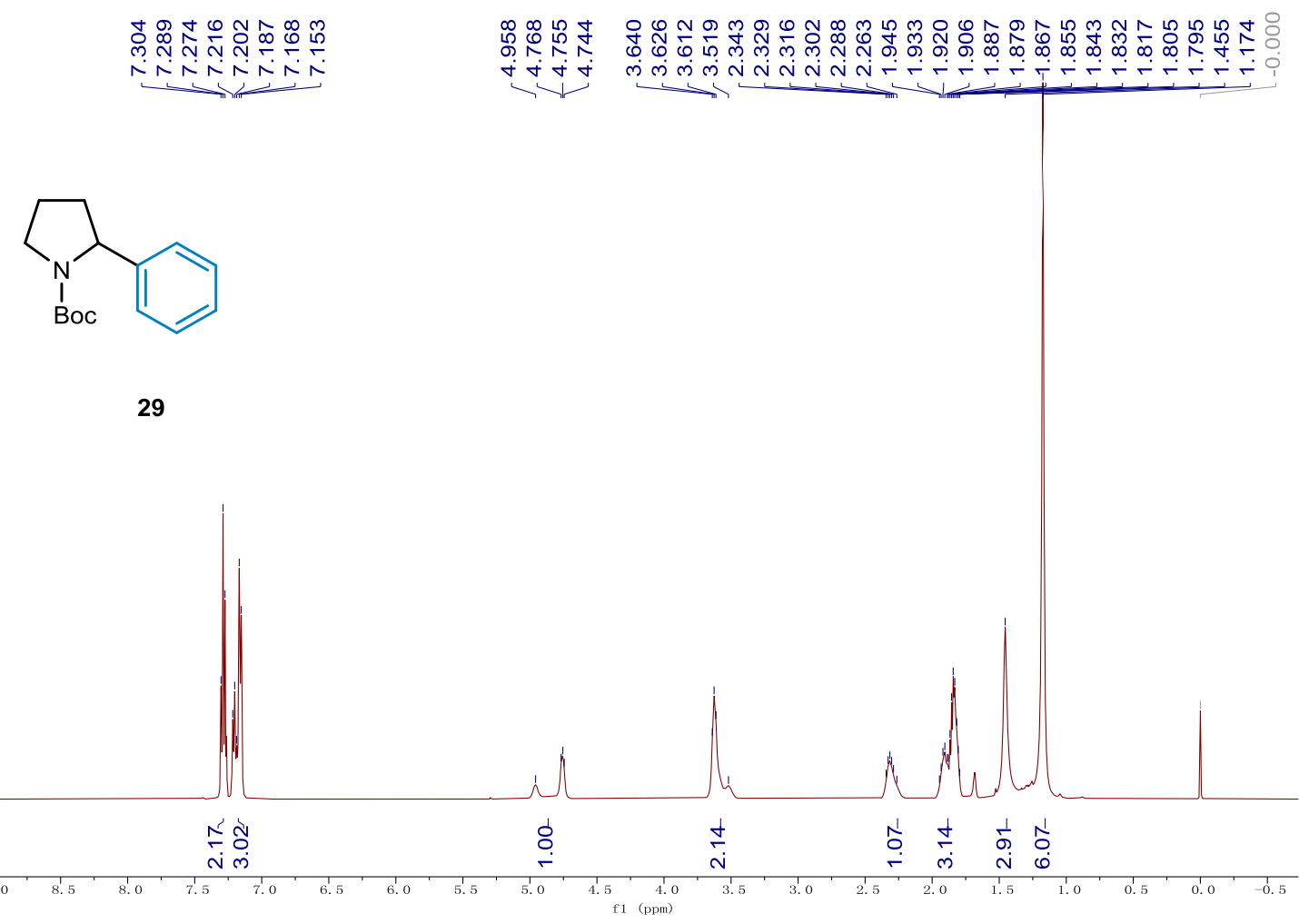


${ }^{13} \mathrm{C}$ NMR $125 \mathrm{MHz}, \mathrm{CDCl}_{3}$
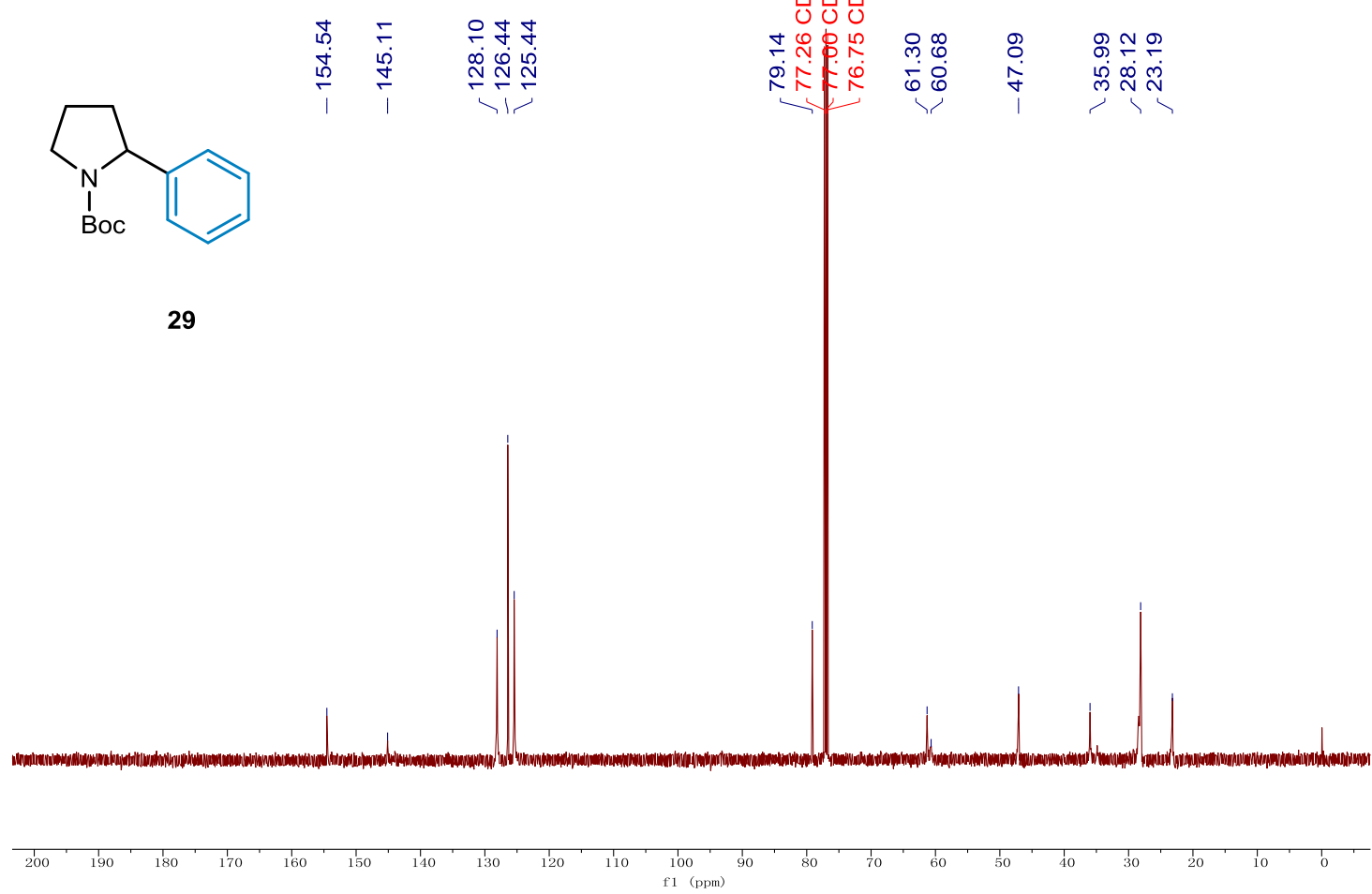

${ }^{13} \mathrm{C}$ NMR $125 \mathrm{MHz},\left(\mathrm{CD}_{3}\right)_{2} \mathrm{CO}$
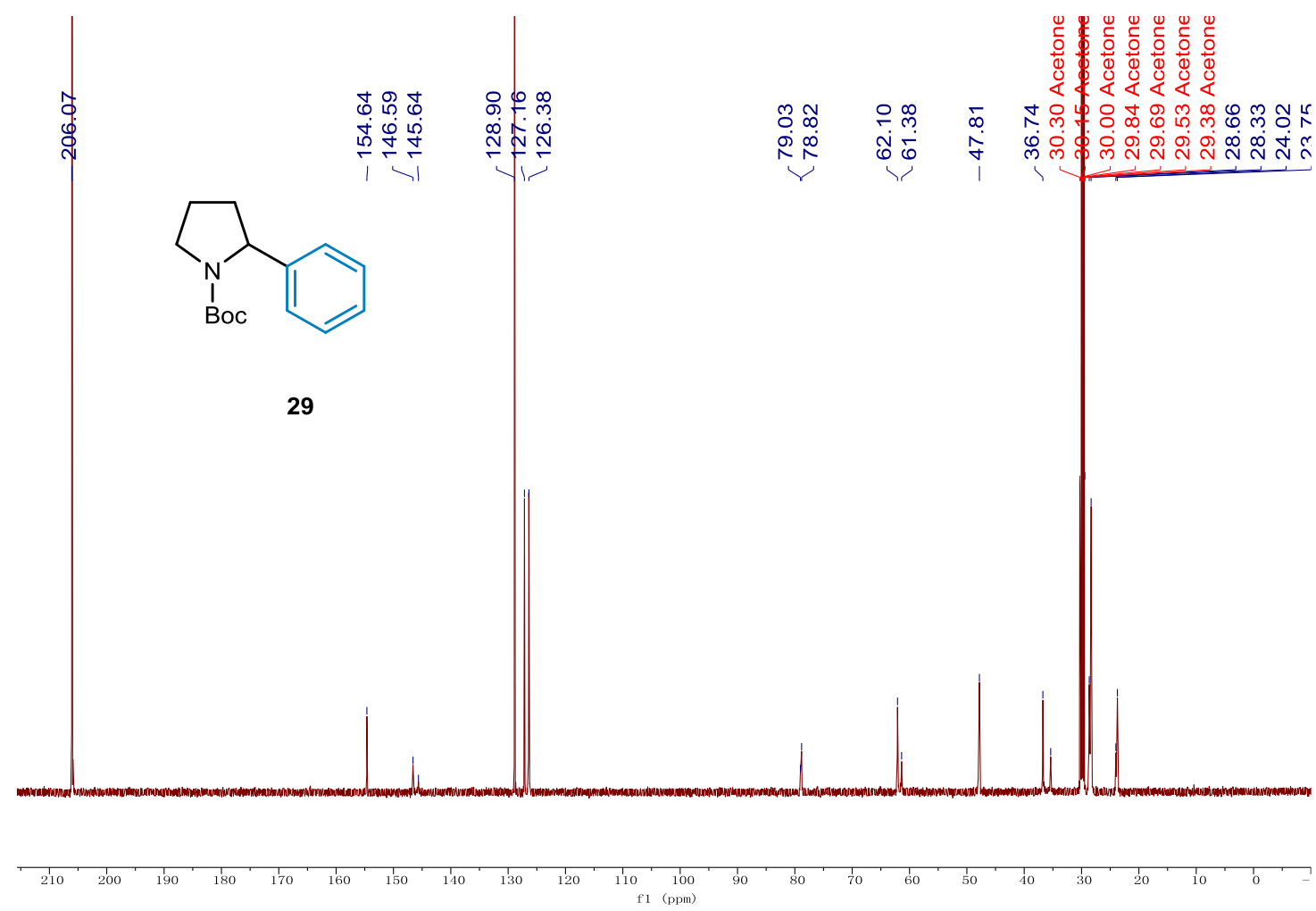
${ }^{1} \mathrm{H}$ NMR $500 \mathrm{MHz}, \mathrm{CDCl}_{3}$

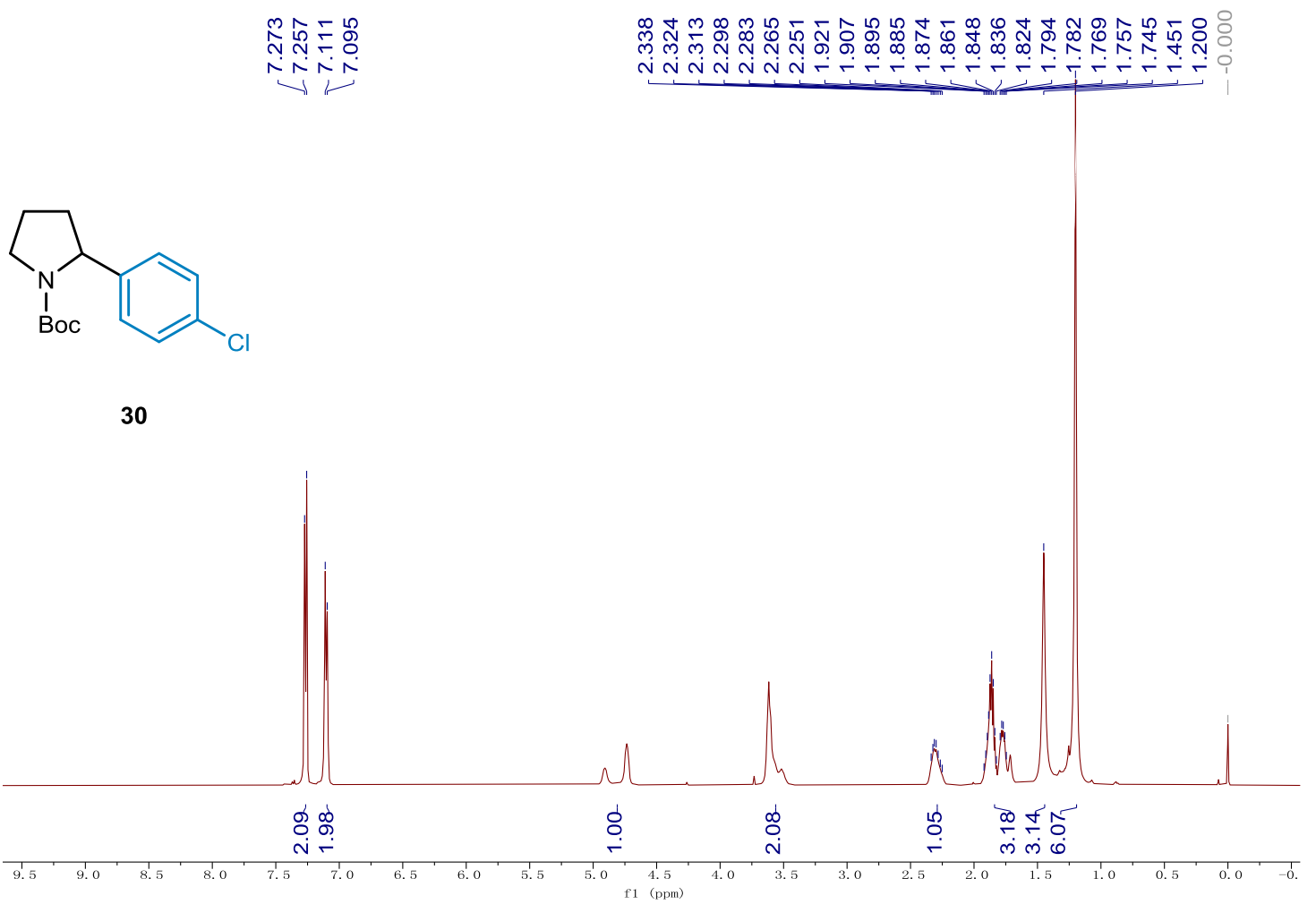

${ }^{13} \mathrm{C}$ NMR $125 \mathrm{MHz}, \mathrm{CDCl}_{3}$
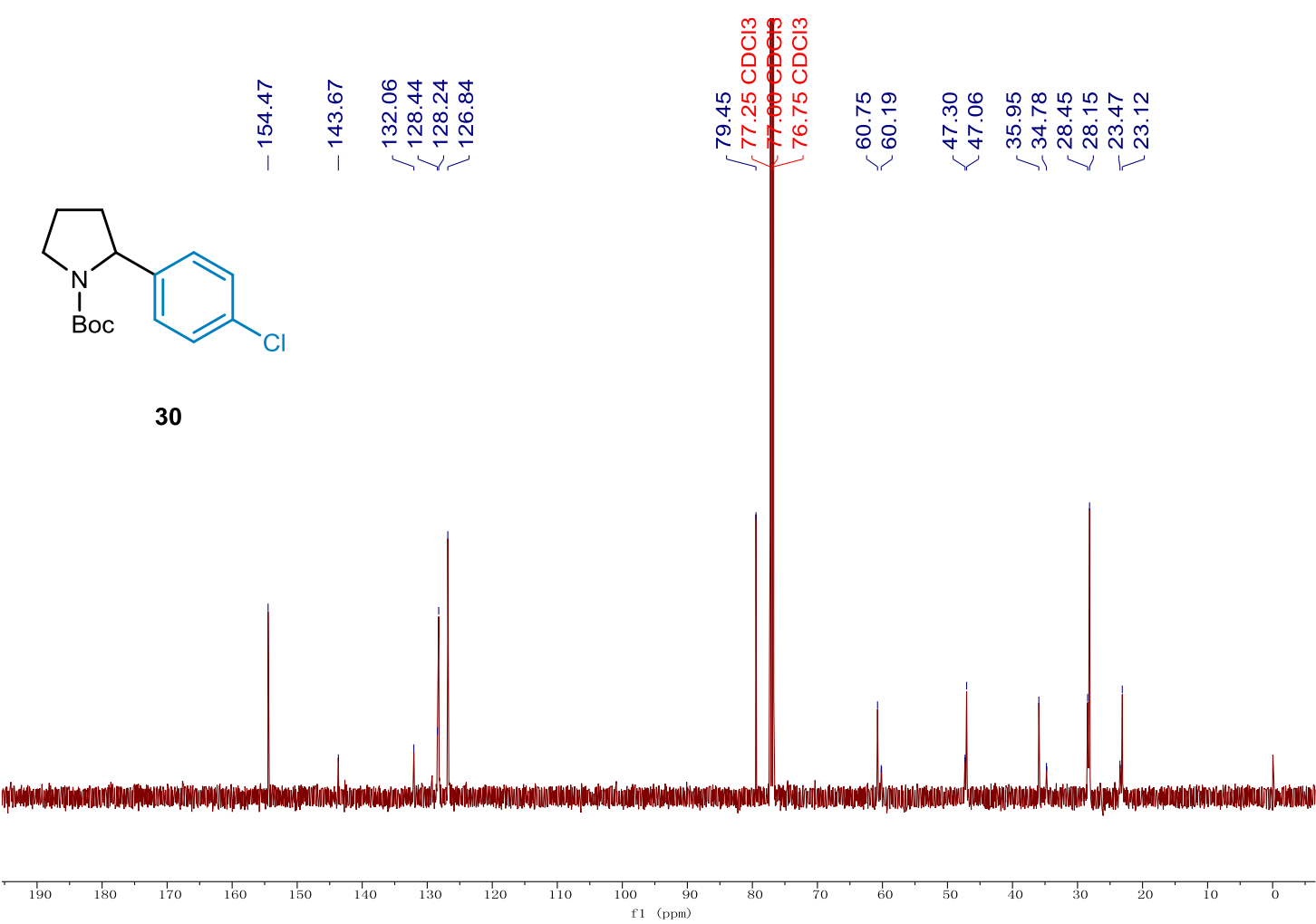
${ }^{1} \mathrm{H}$ NMR $500 \mathrm{MHz}, \mathrm{CDCl}_{3}$

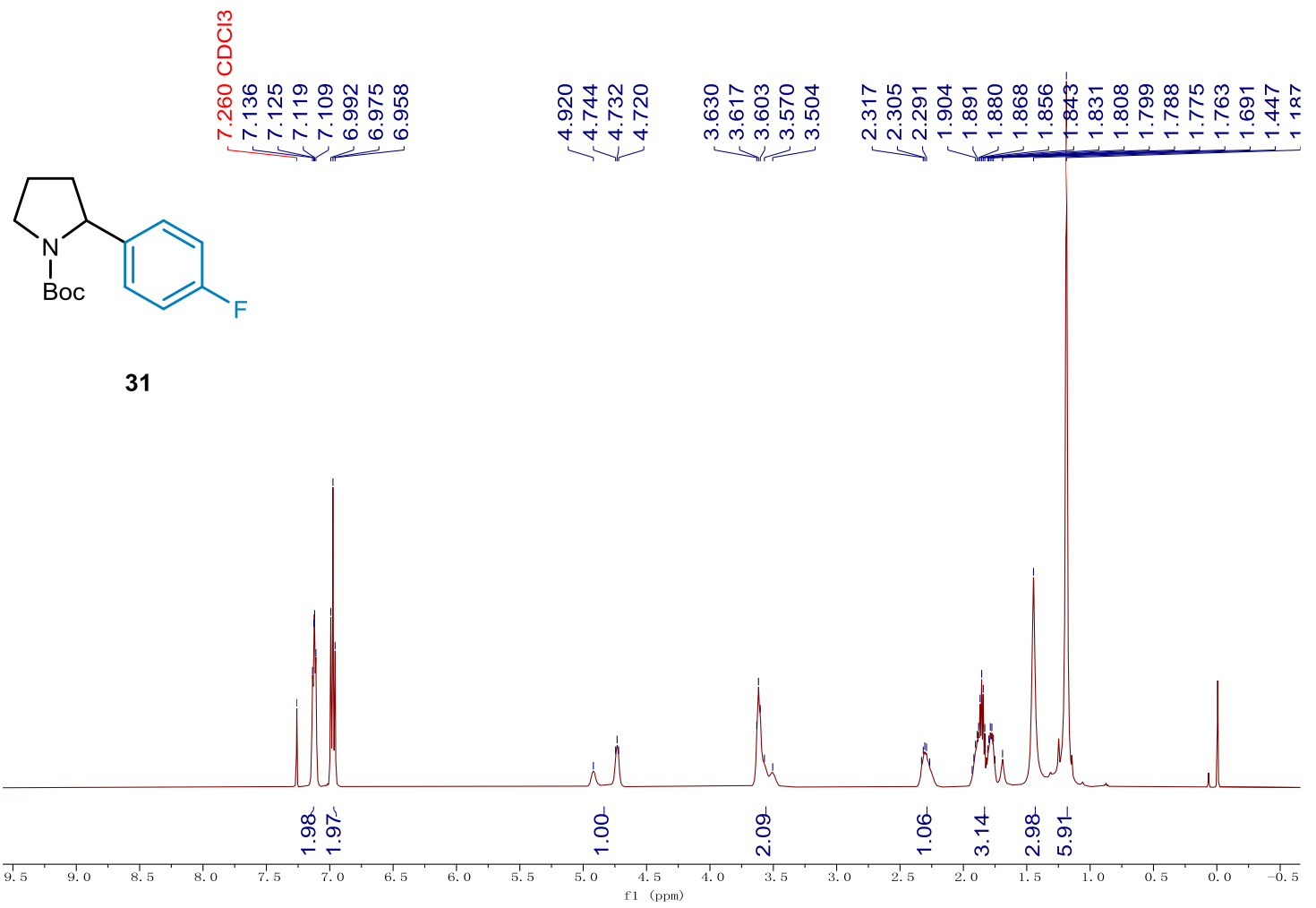

${ }^{13} \mathrm{C}$ NMR $125 \mathrm{MHz}, \mathrm{CDCl}_{3}$
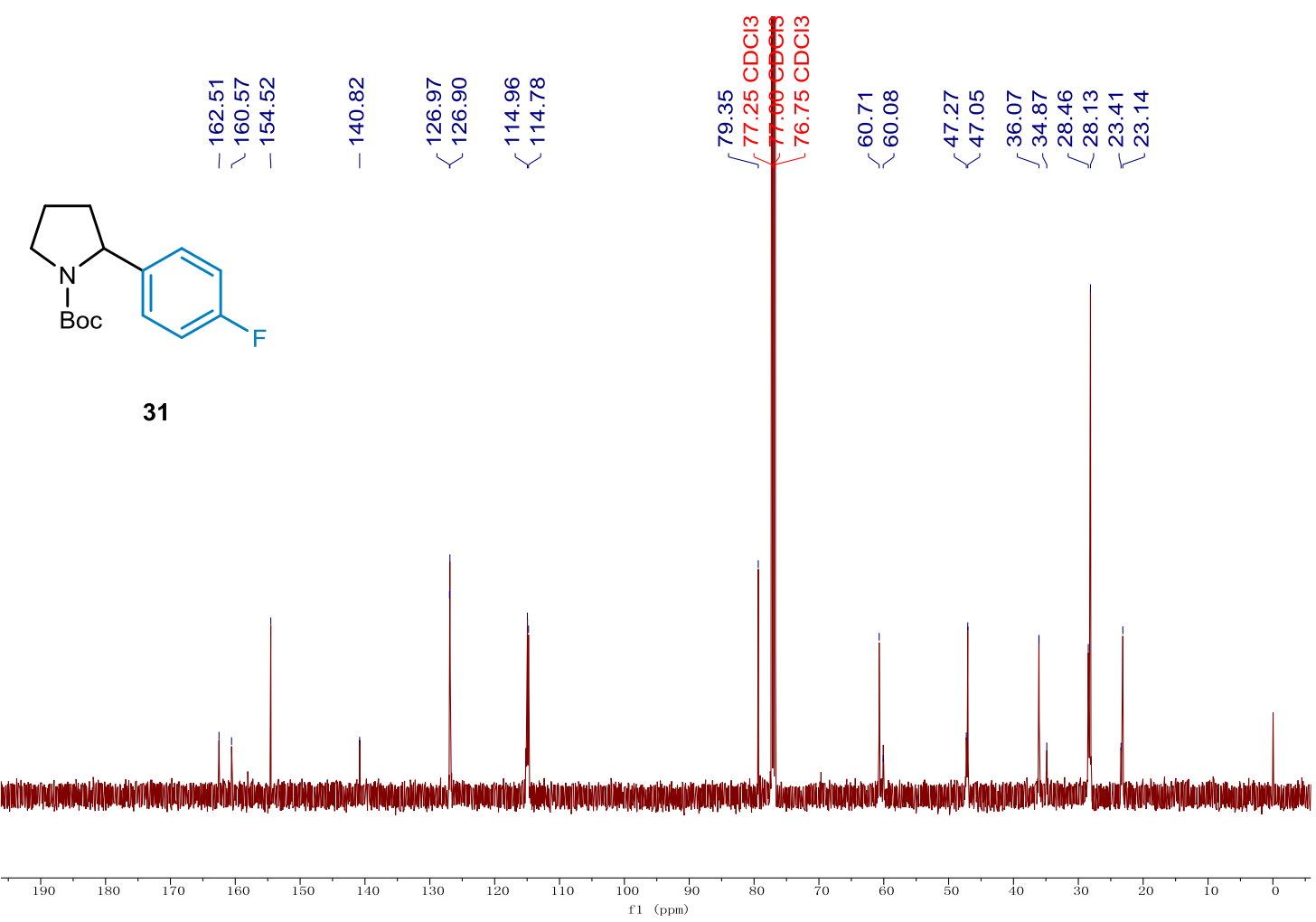
${ }^{19} \mathrm{~F}$ NMR $470 \mathrm{MHz}, \mathrm{CDCl}_{3}$

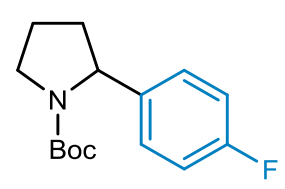

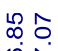

ํㅜㄷ

31 
${ }^{13} \mathrm{C}$ NMR $125 \mathrm{MHz}, \mathrm{CDCl}_{3}$

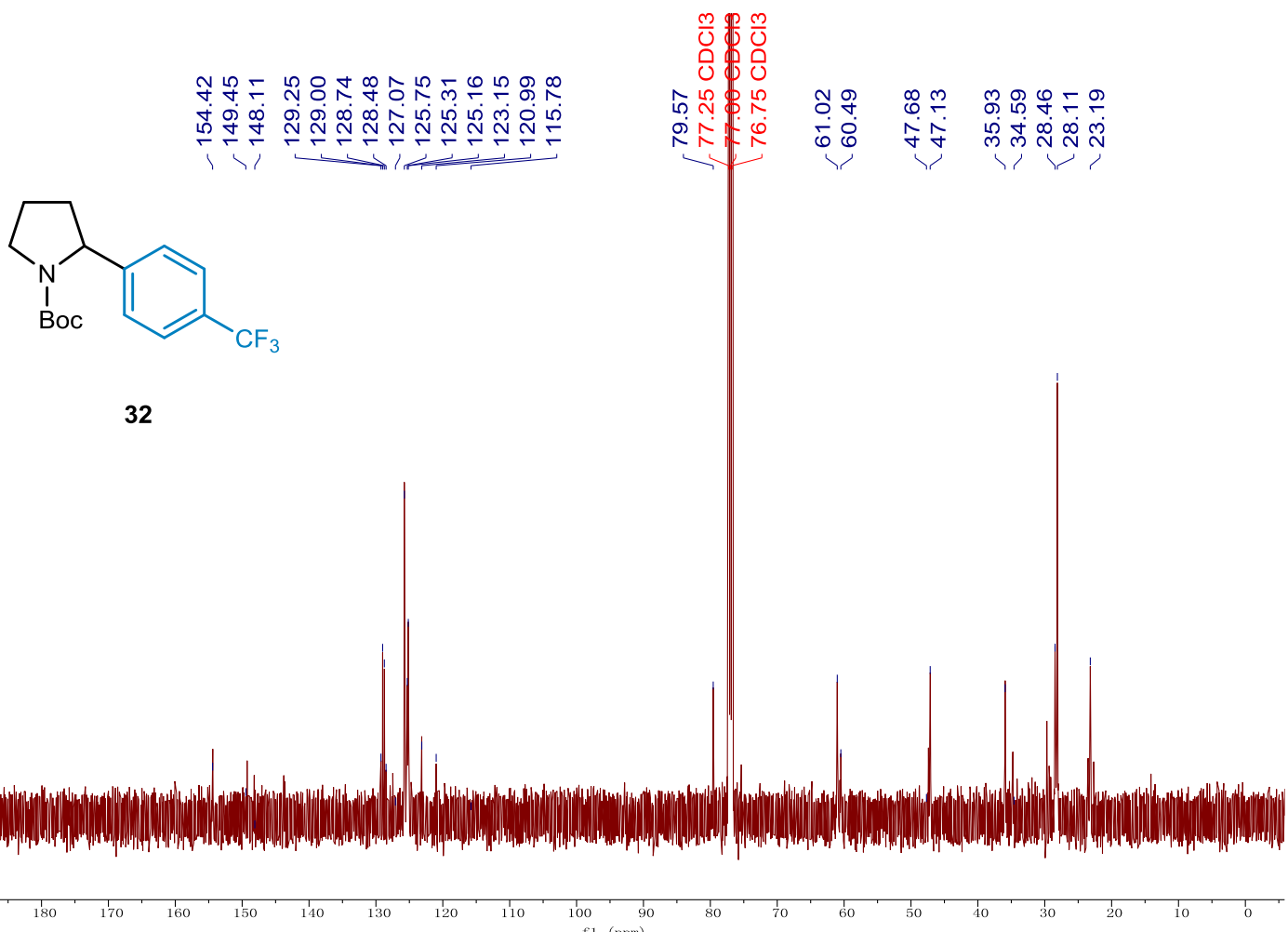

${ }^{19} \mathrm{~F}$ NMR $470 \mathrm{MHz}, \mathrm{CDCl}_{3}$ 


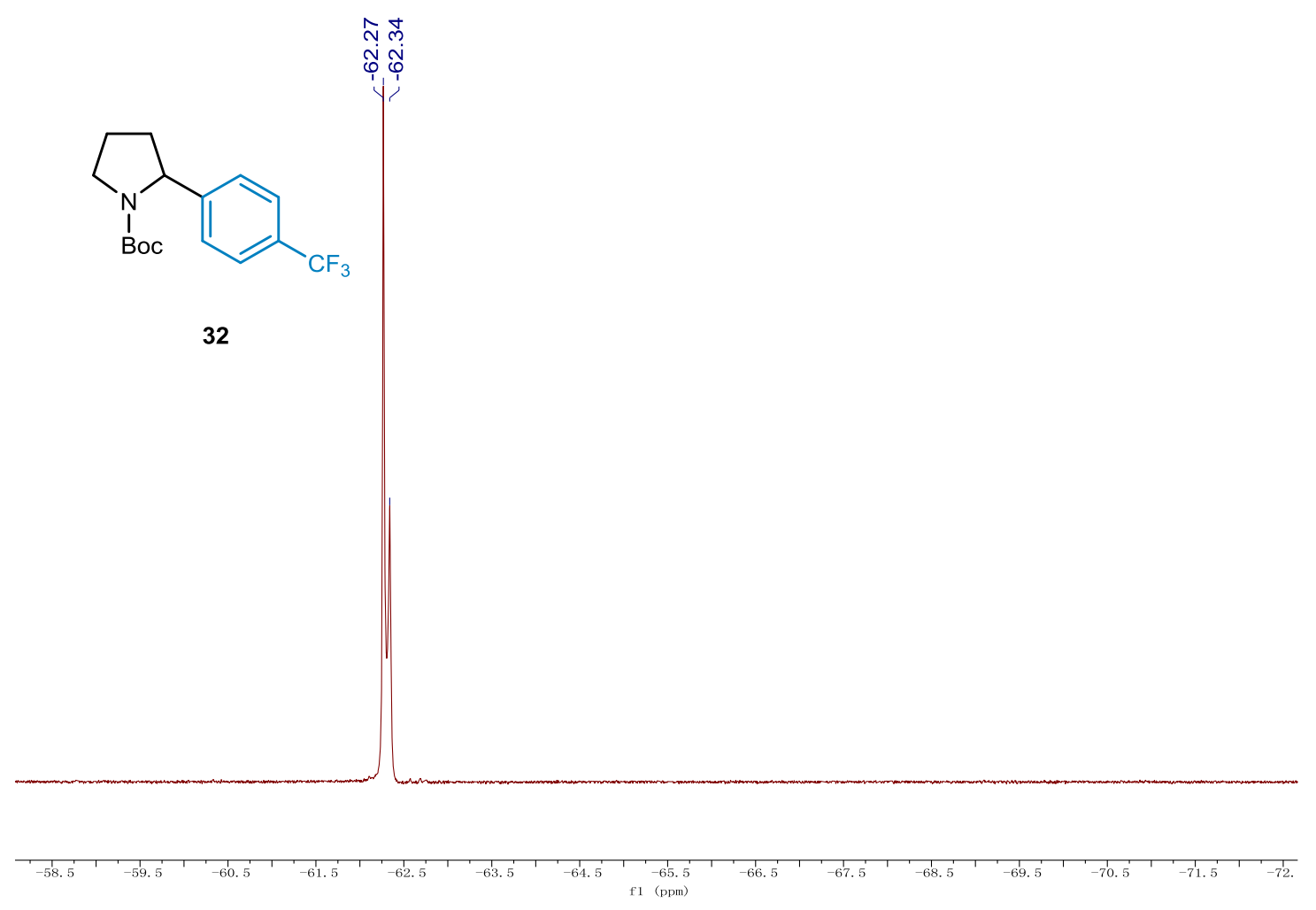

${ }^{1} \mathrm{H}$ NMR $500 \mathrm{MHz}, \mathrm{CDCl}_{3}$ 


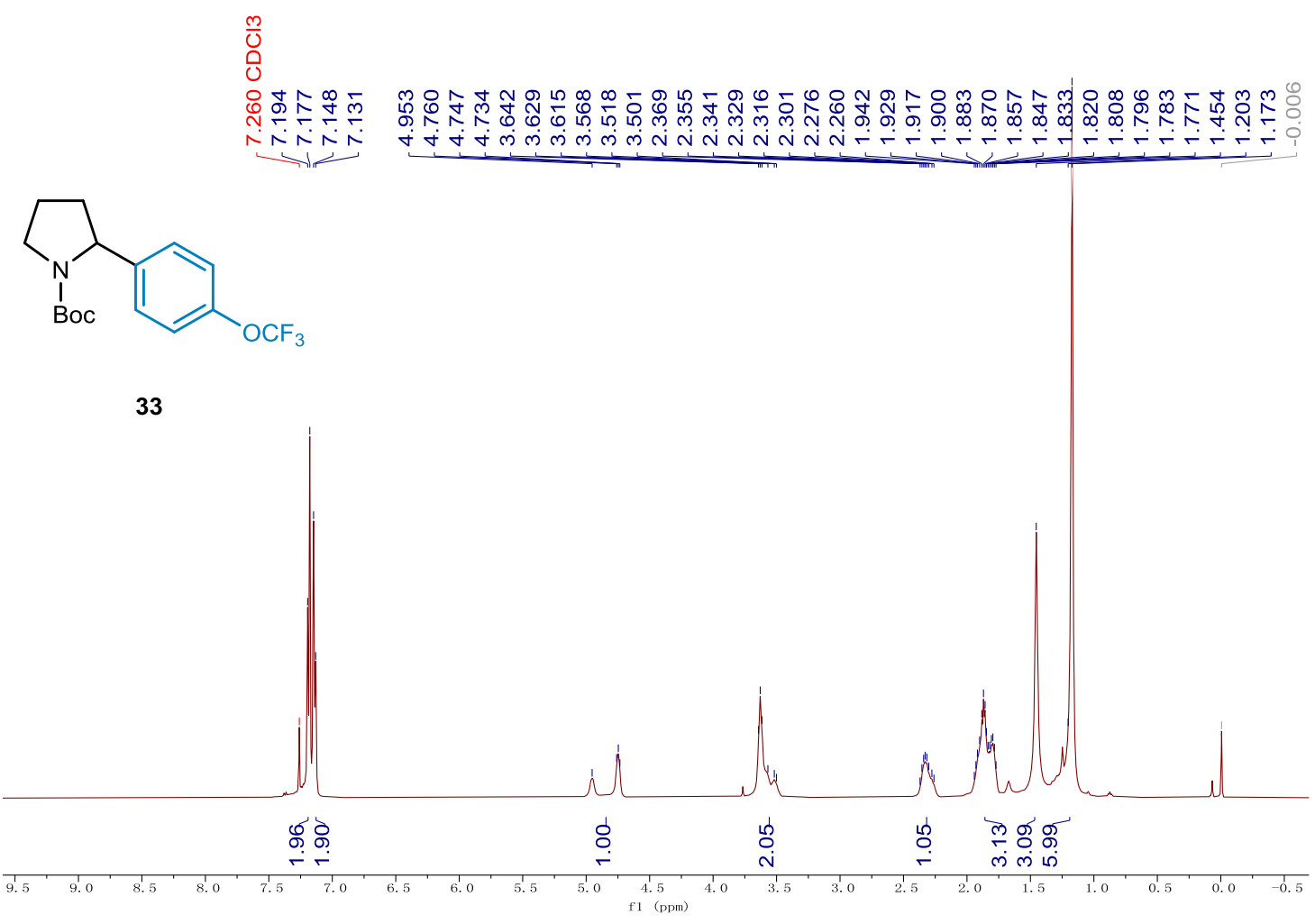

${ }^{13} \mathrm{C} \mathrm{NMR} 125 \mathrm{MHz}, \mathrm{CDCl}_{3}$
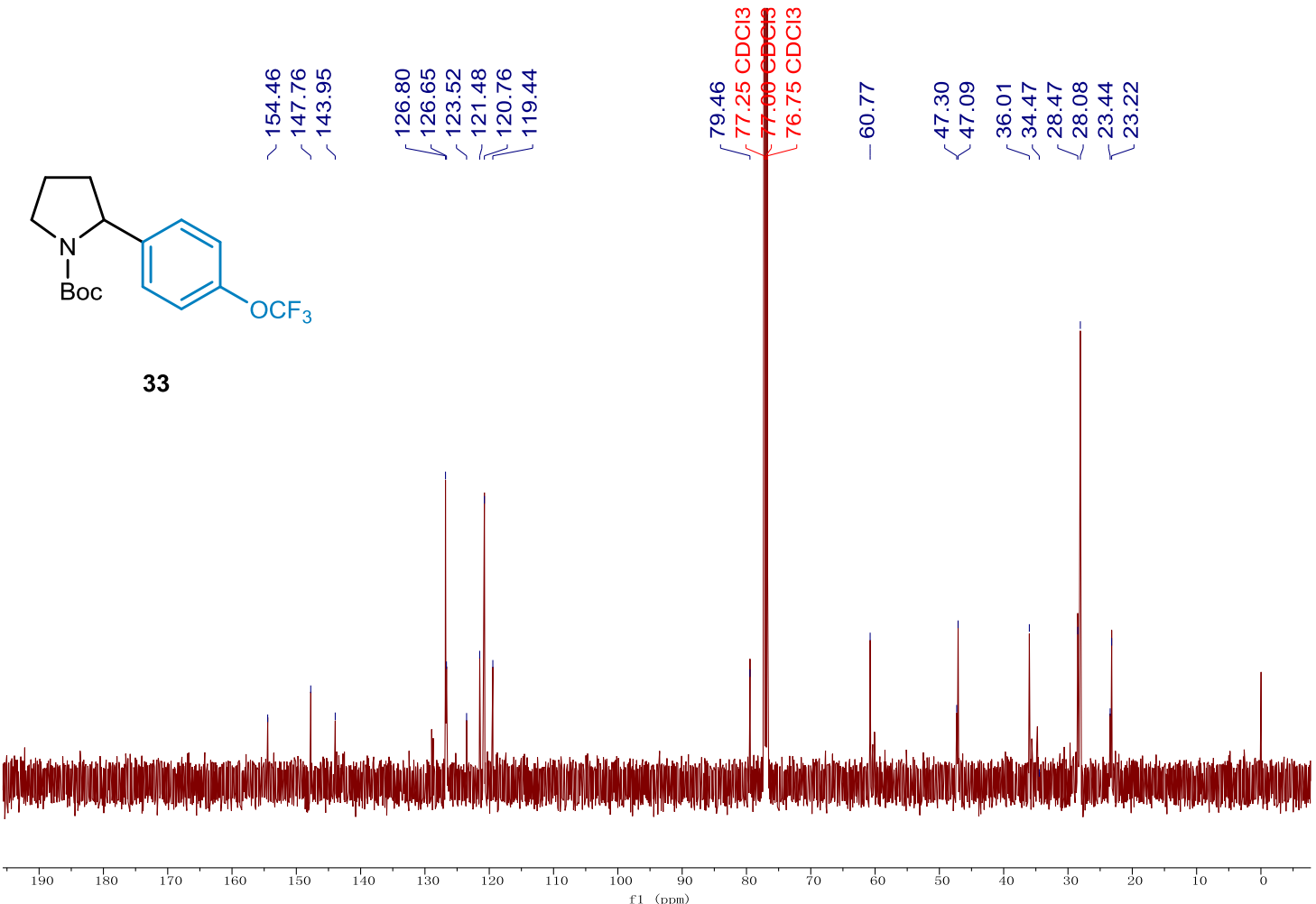

${ }^{19} \mathrm{~F}$ NMR $470 \mathrm{MHz}, \mathrm{CDCl}_{3}$ 


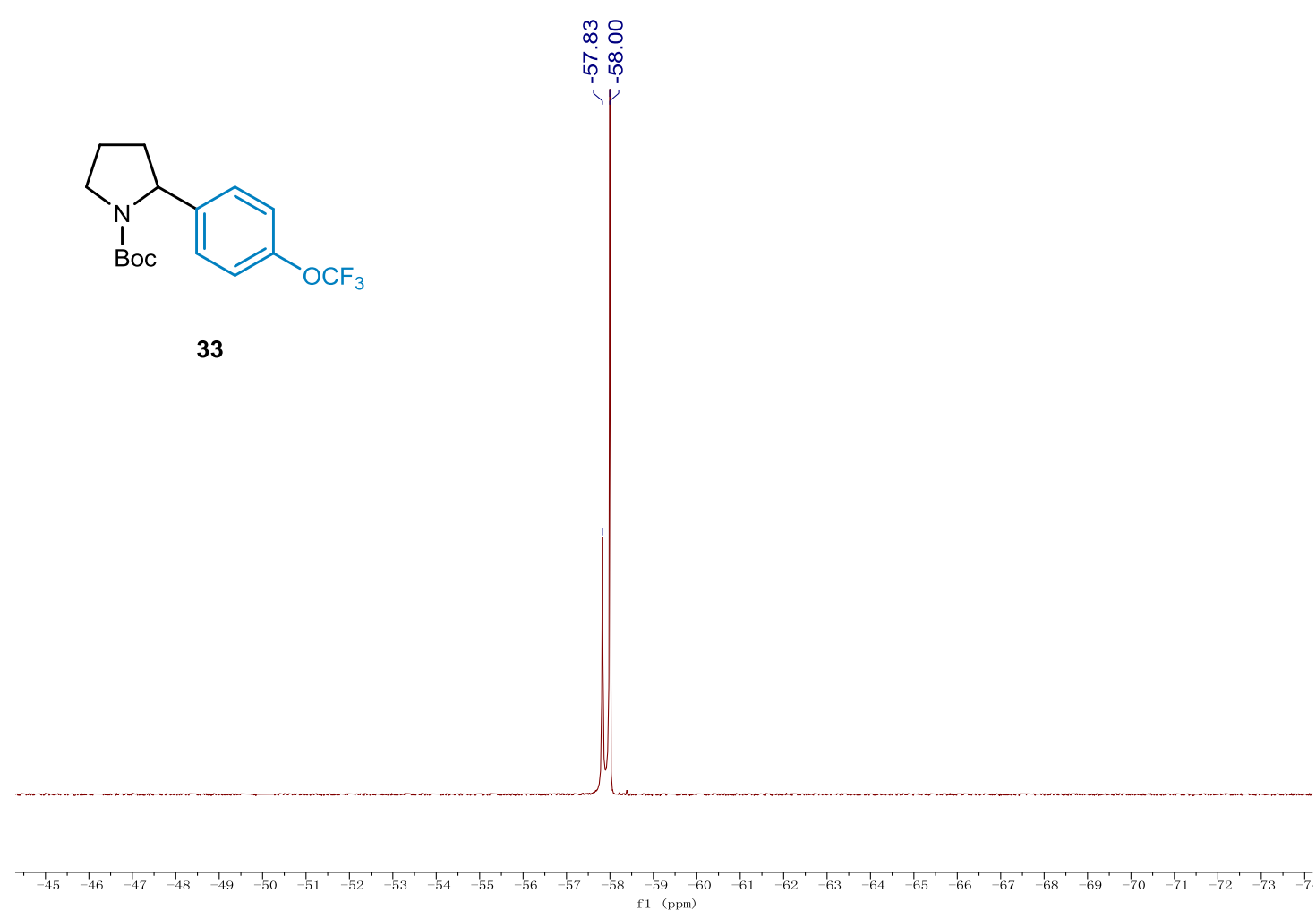

${ }^{1} \mathrm{H} \mathrm{NMR} 500 \mathrm{MHz}, \mathrm{CDCl}_{3}$ 


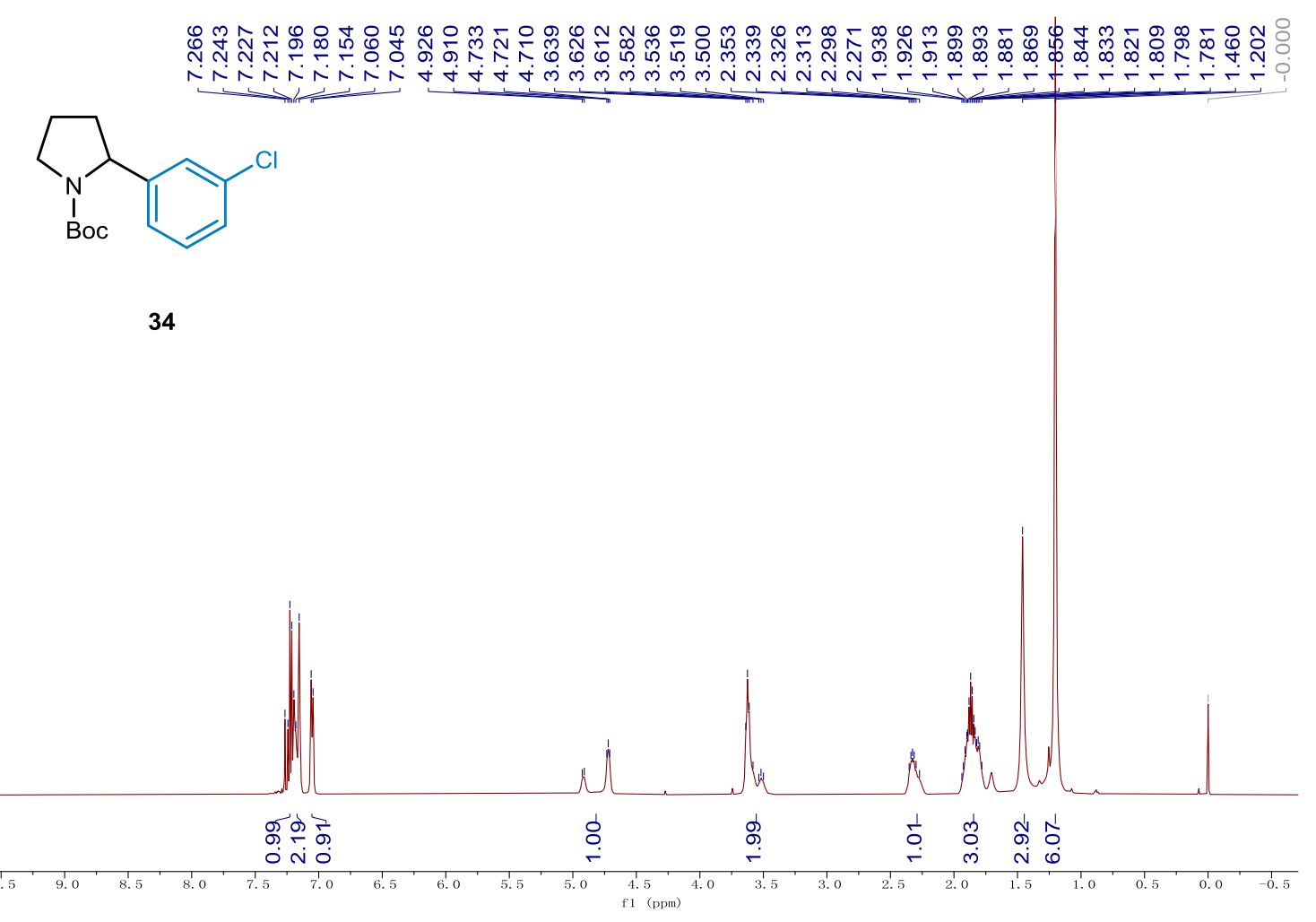

${ }^{13} \mathrm{C}$ NMR $125 \mathrm{MHz}, \mathrm{CDCl}_{3}$

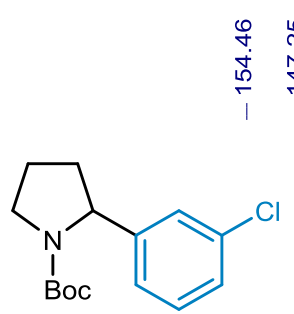

34

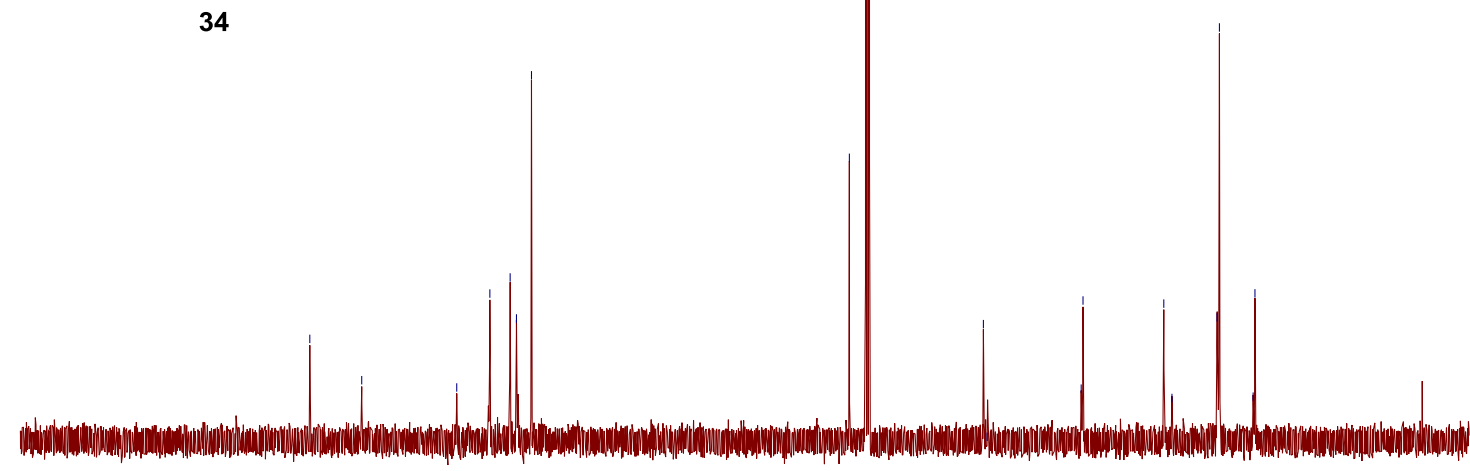

${ }^{1} \mathrm{H} \mathrm{NMR} 500 \mathrm{MHz}, \mathrm{CDCl}_{3}$ 
<smiles>O=C(c1ccccc1)N1CCCC1c1ccccc1F</smiles>

35

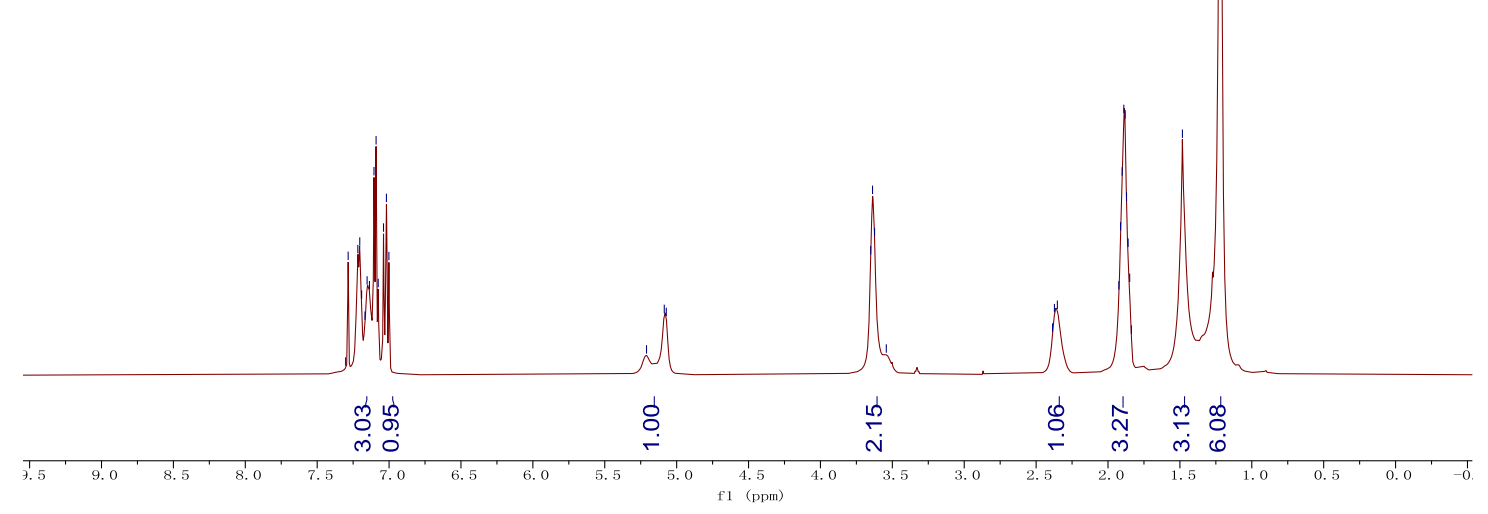

${ }^{13} \mathrm{C} \mathrm{NMR} 125 \mathrm{MHz}, \mathrm{CDCl}_{3}$

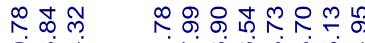

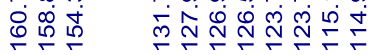

i,<smiles>O=C(c1ccccc1)N1CCCC1c1ccccc1F</smiles>

35
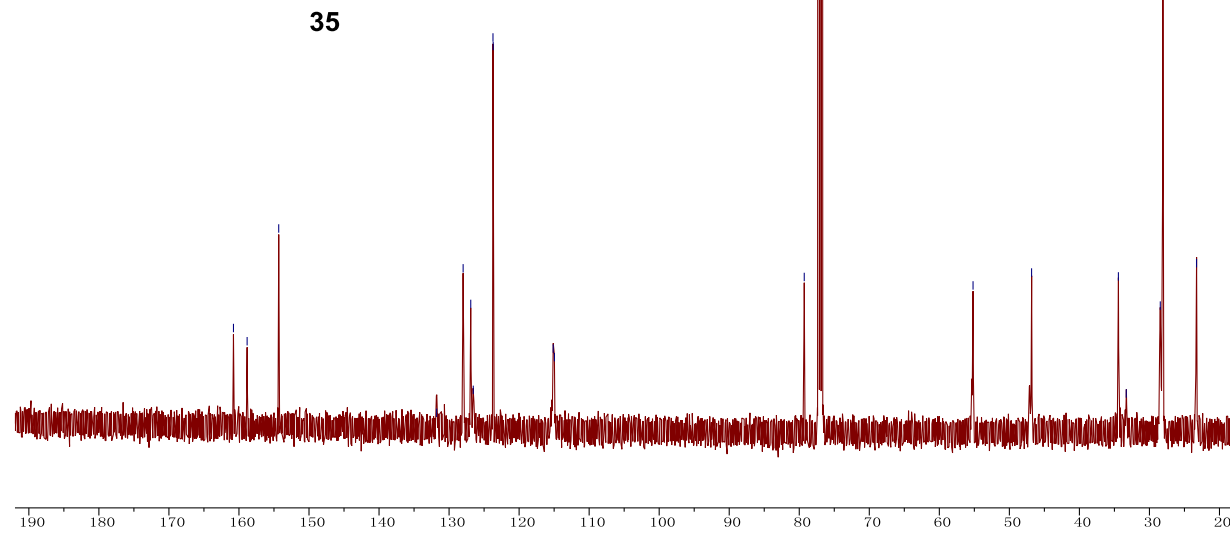

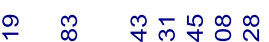

in

${ }^{19} \mathrm{~F}$ NMR $470 \mathrm{MHz}, \mathrm{CDCl}_{3}$ 


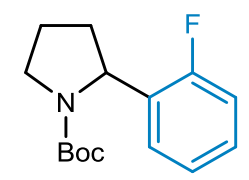

35

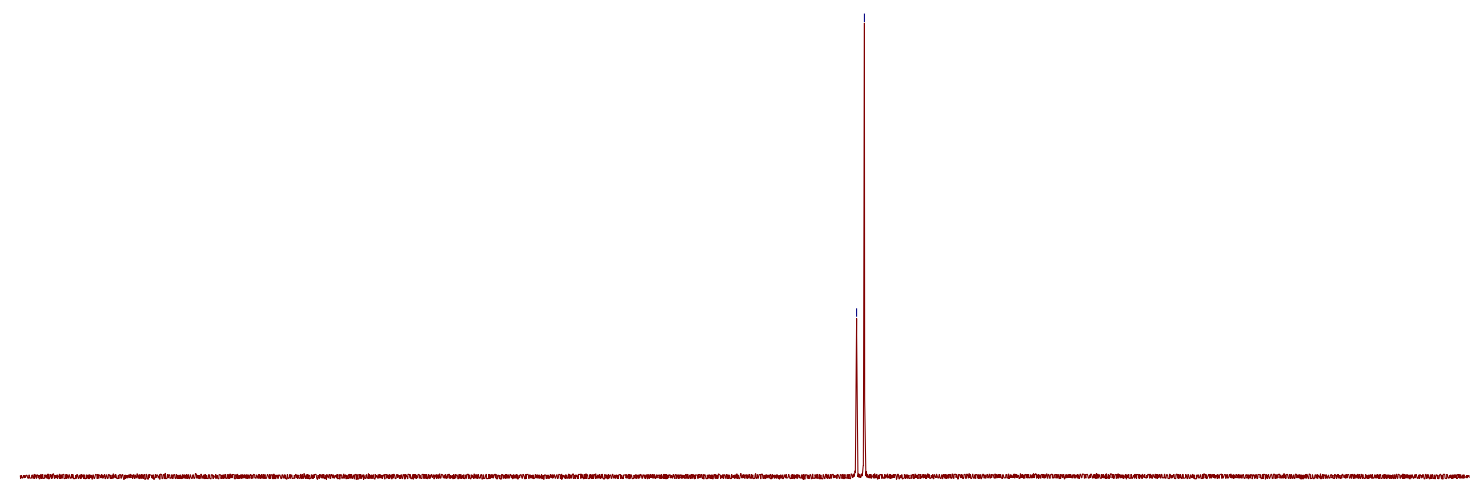

\begin{tabular}{l}
20 \\
\hline $0^{\prime}$
\end{tabular}

${ }^{1} \mathrm{H}$ NMR $500 \mathrm{MHz}, \mathrm{CDCl}_{3}$ 


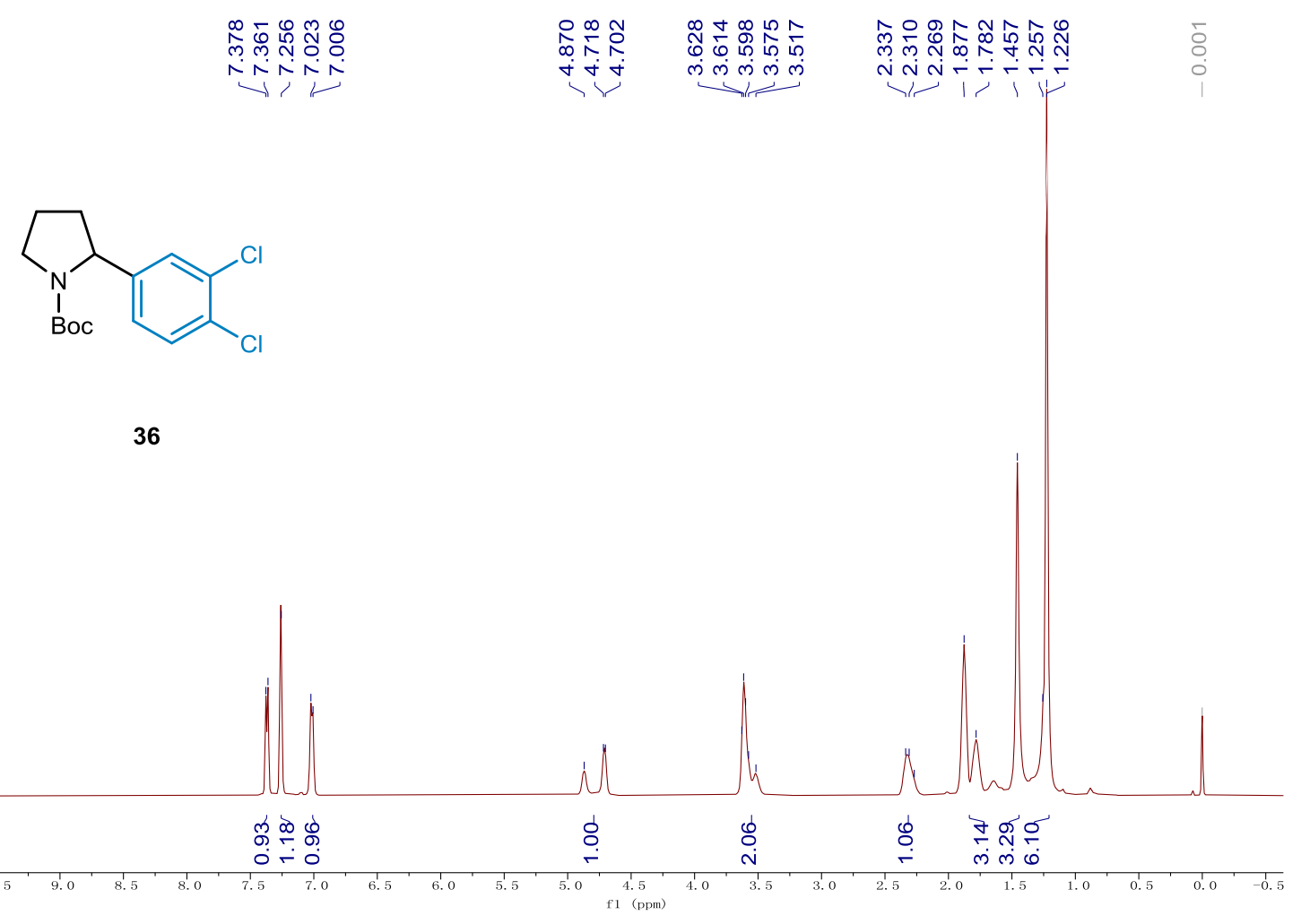

${ }^{13} \mathrm{C}$ NMR $125 \mathrm{MHz}, \mathrm{CDCl}_{3}$

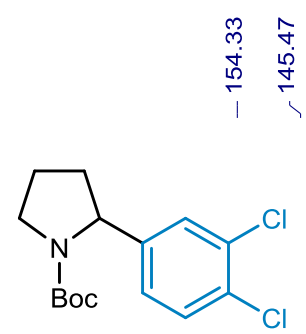

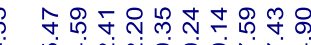

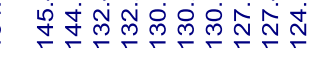

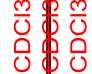

유유.

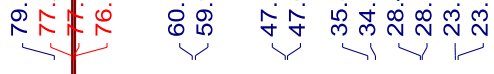

36

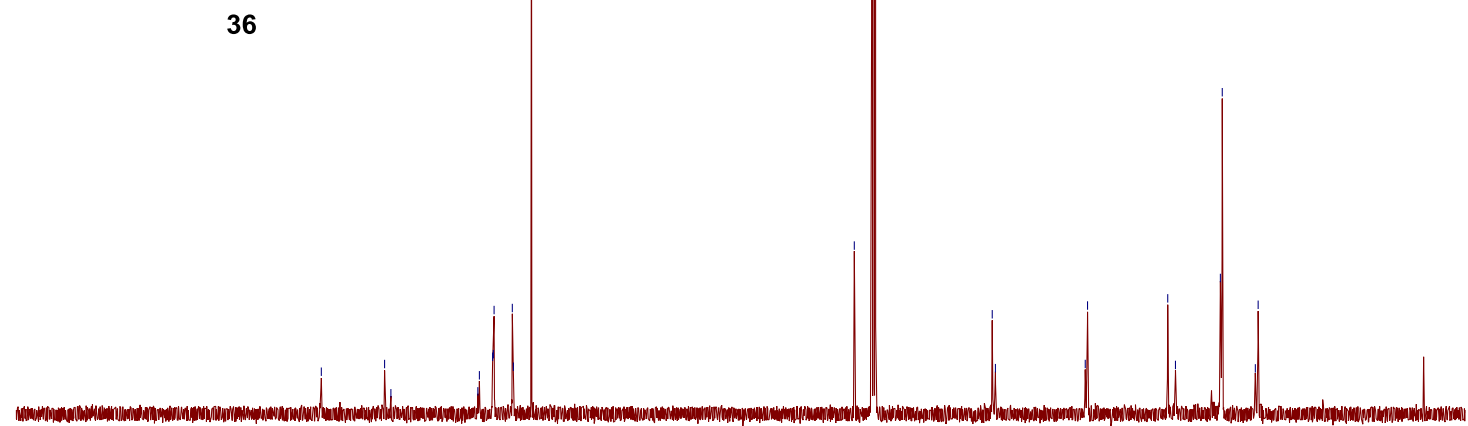

${ }^{1} \mathrm{H} \mathrm{NMR} 500 \mathrm{MHz}, \mathrm{CDCl}_{3}$ 


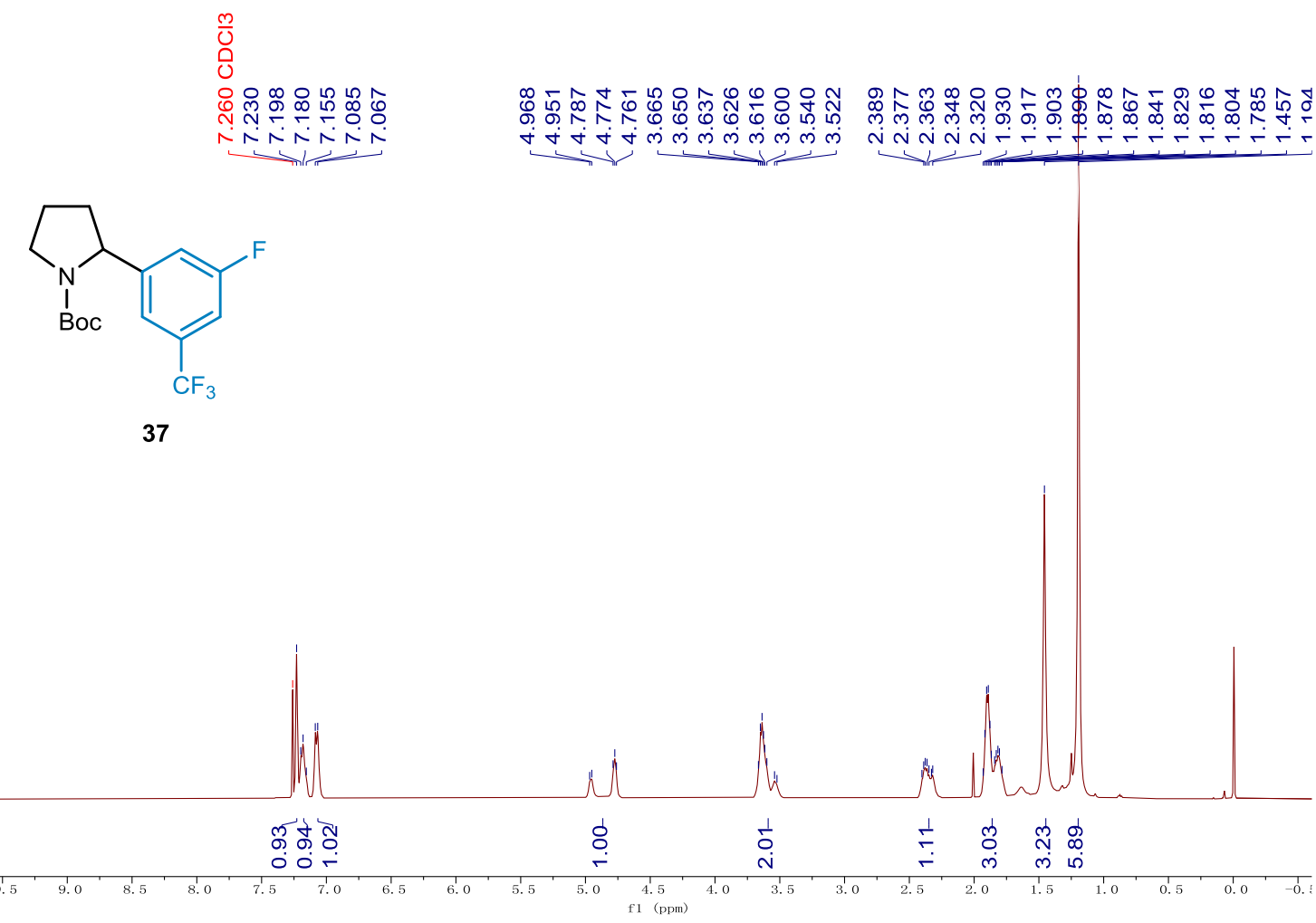

${ }^{13} \mathrm{C} \mathrm{NMR} 125 \mathrm{MHz}, \mathrm{CDCl}_{3}$
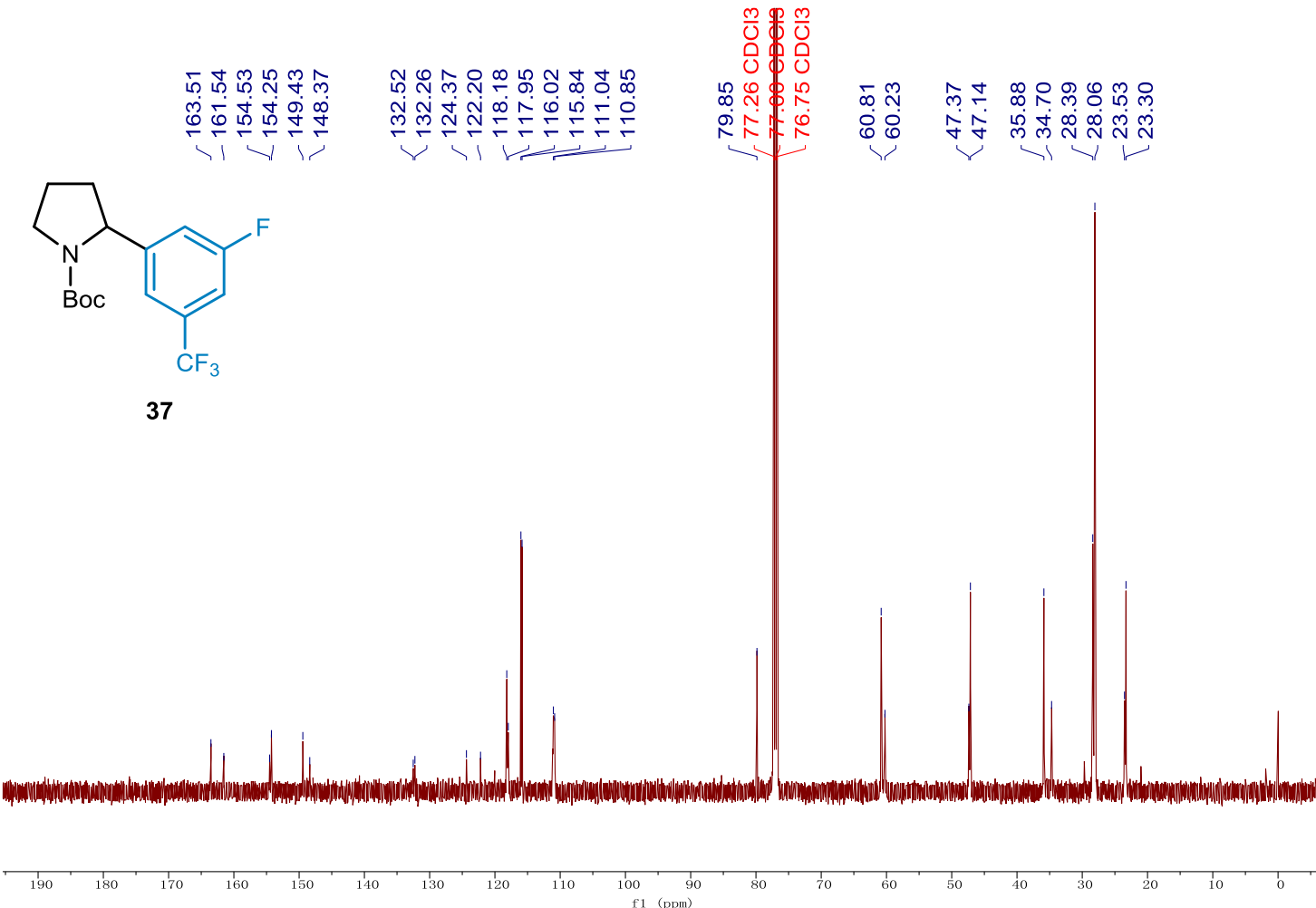

${ }^{19} \mathrm{~F}$ NMR $470 \mathrm{MHz}, \mathrm{CDCl}_{3}$ 


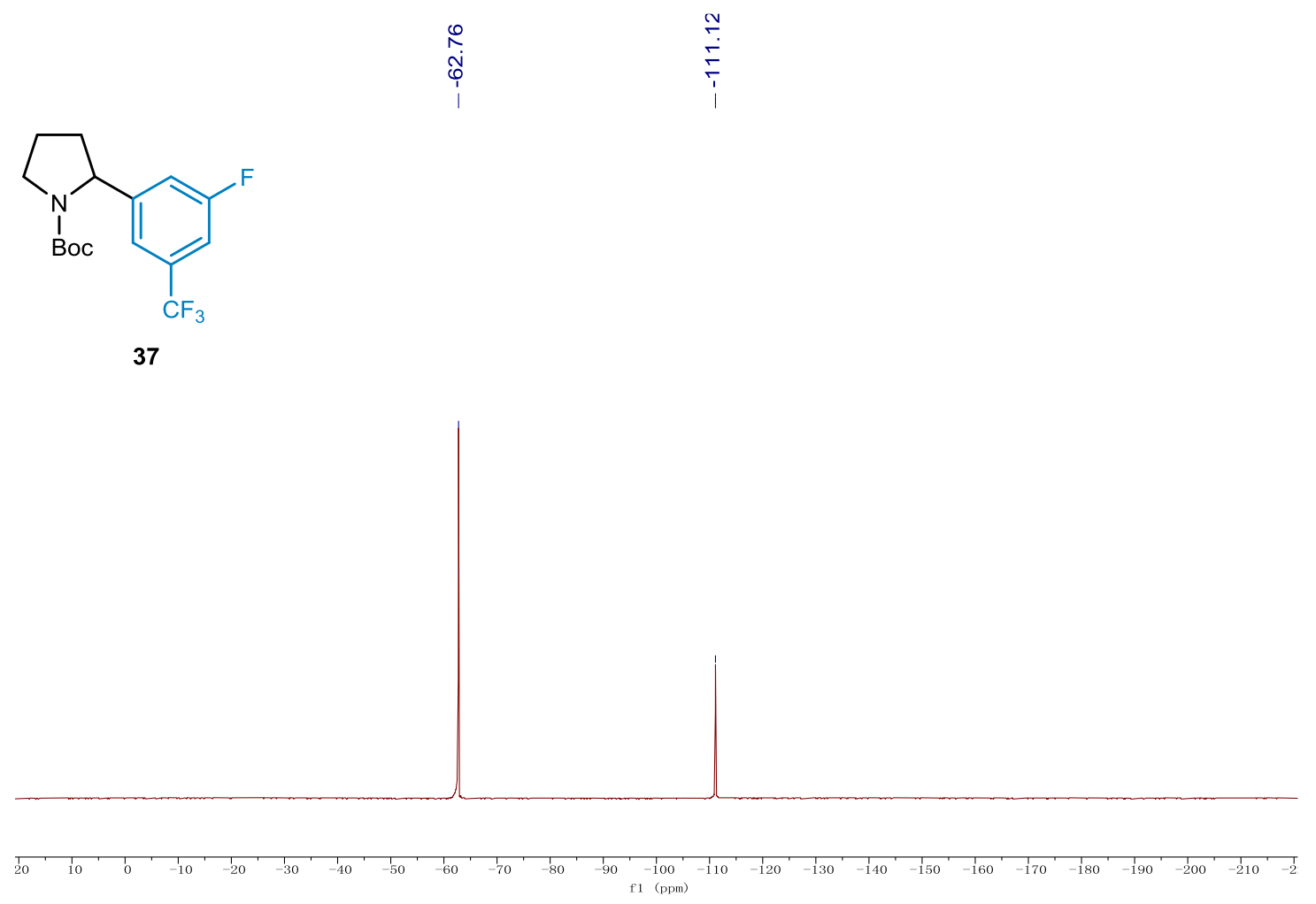

${ }^{1} \mathrm{H}$ NMR $500 \mathrm{MHz}, \mathrm{CDCl}_{3}$ 
鱼

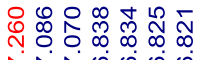

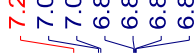

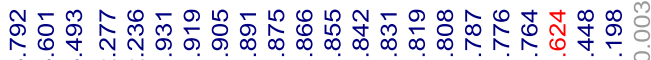

लि

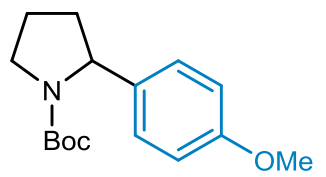

38

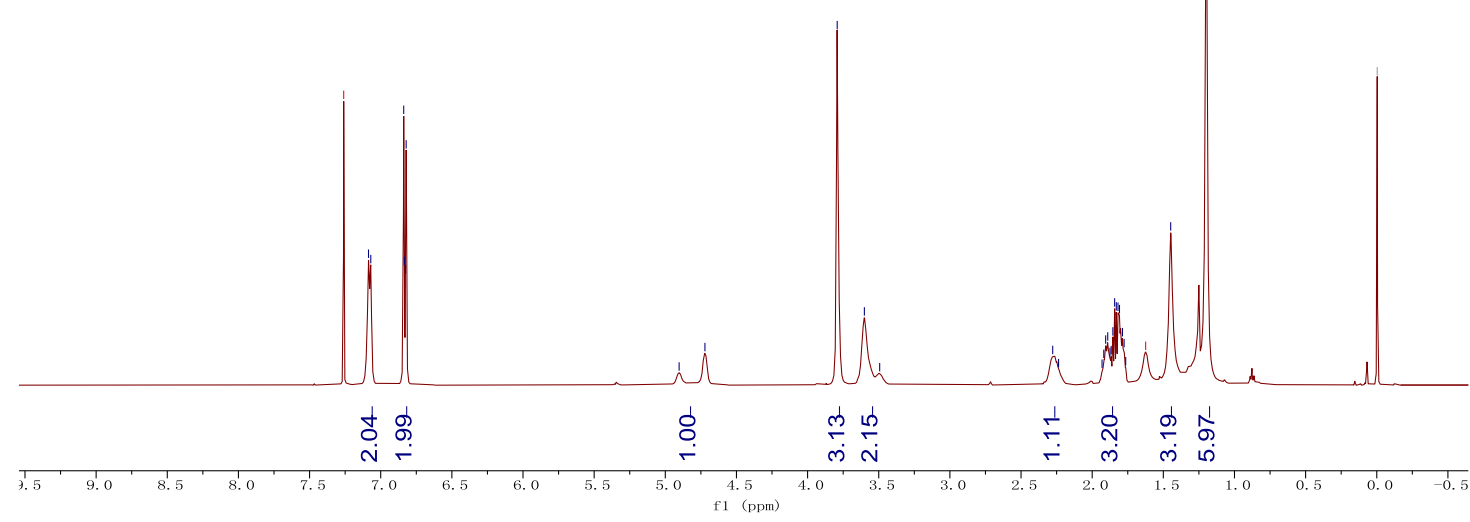

${ }^{13} \mathrm{C}$ NMR $125 \mathrm{MHz}, \mathrm{CDCl}_{3}$

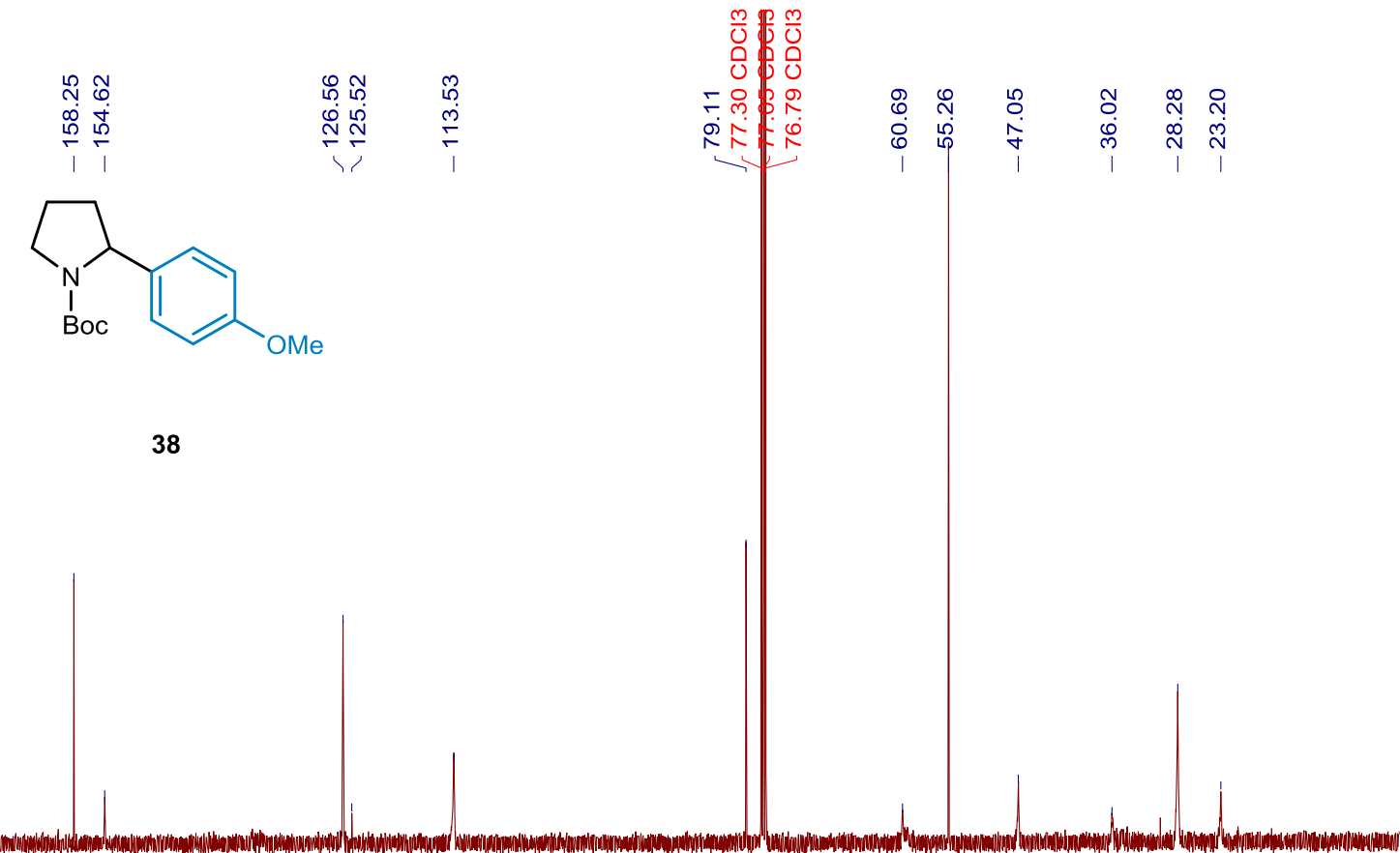

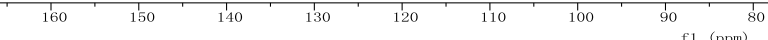

${ }^{1} \mathrm{H}$ NMR $500 \mathrm{MHz}, \mathrm{CDCl}_{3}$ 
吕

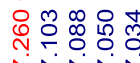

iñis

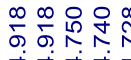

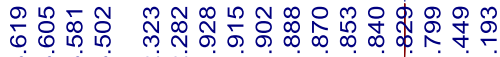

$\sqrt{.24}$

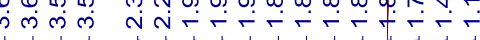

Boc

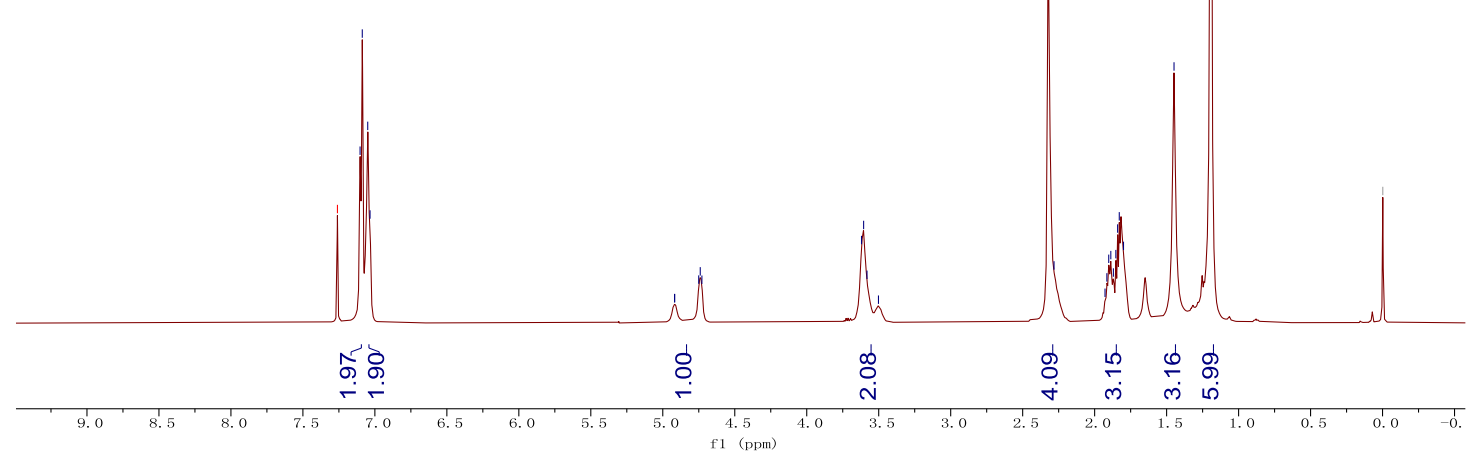

${ }^{13} \mathrm{C}$ NMR $125 \mathrm{MHz}, \mathrm{CDCl}_{3}$

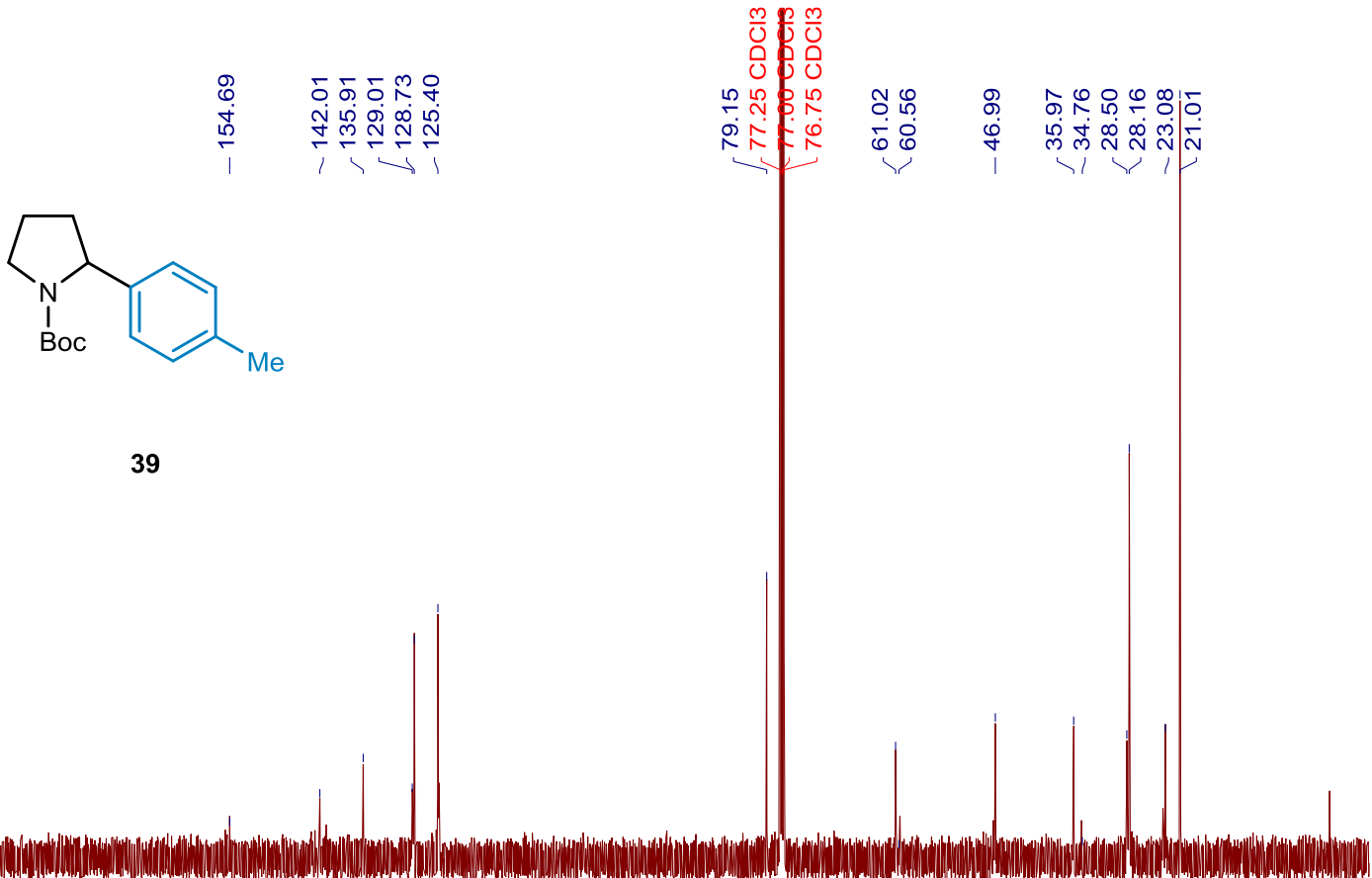

${ }^{1} \mathrm{H} \mathrm{NMR} 500 \mathrm{MHz}, \mathrm{CDCl}_{3}$ 


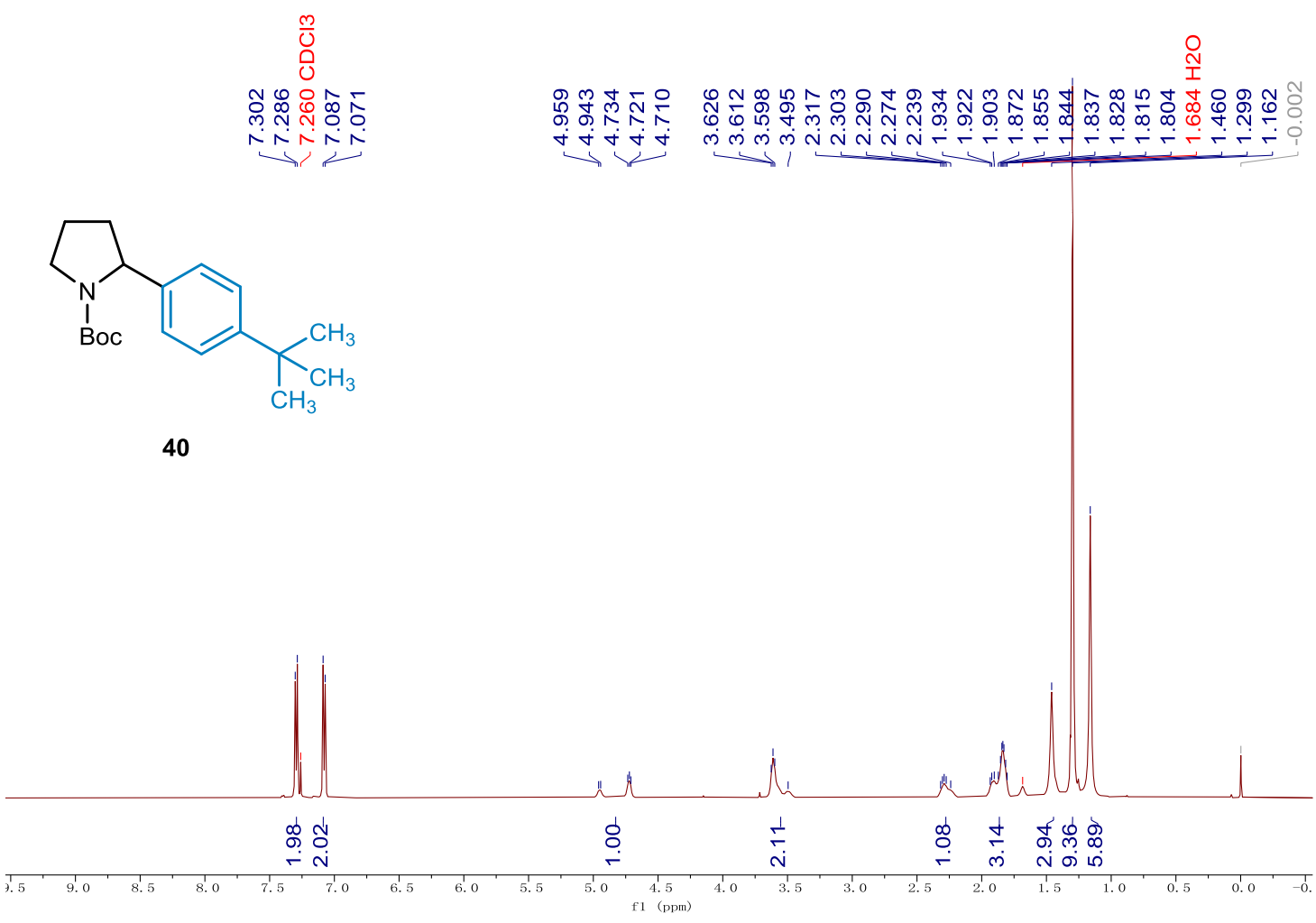

${ }^{13} \mathrm{C} \mathrm{NMR} 125 \mathrm{MHz}, \mathrm{CDCl}_{3}$

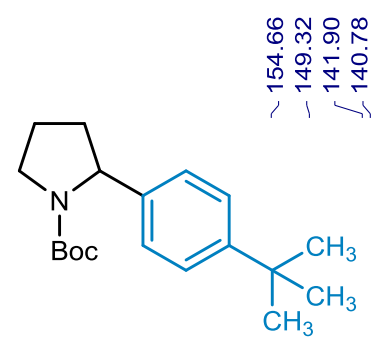

$\stackrel{\infty}{\infty} \underset{\infty}{\infty}$

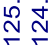

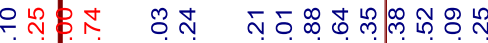

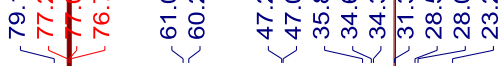

40

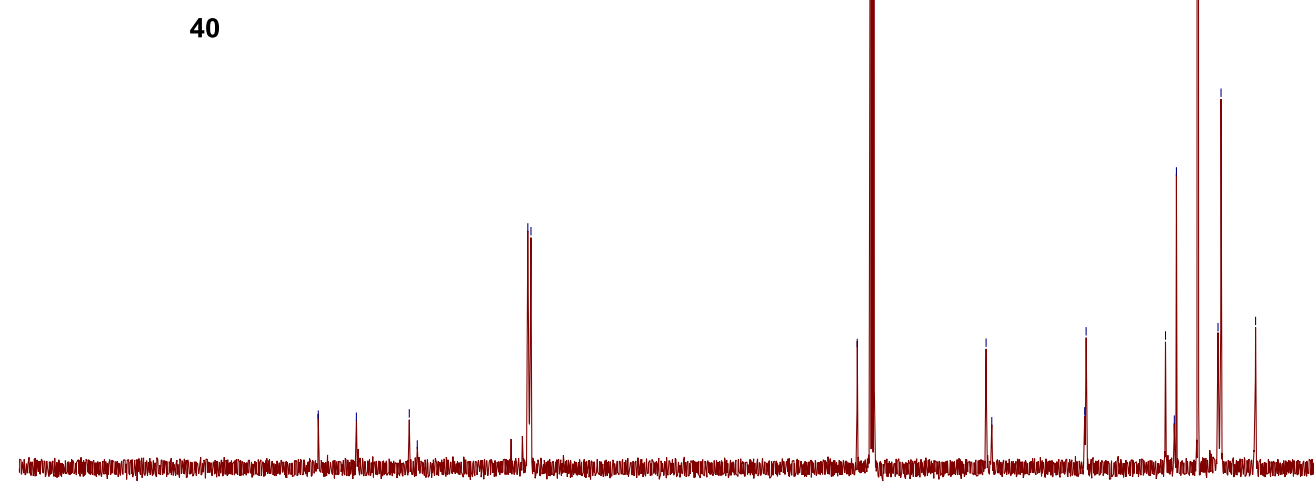

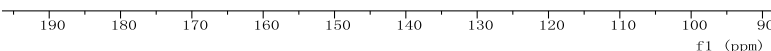

${ }^{1} \mathrm{H}$ NMR $500 \mathrm{MHz}, \mathrm{CDCl}_{3}$ 

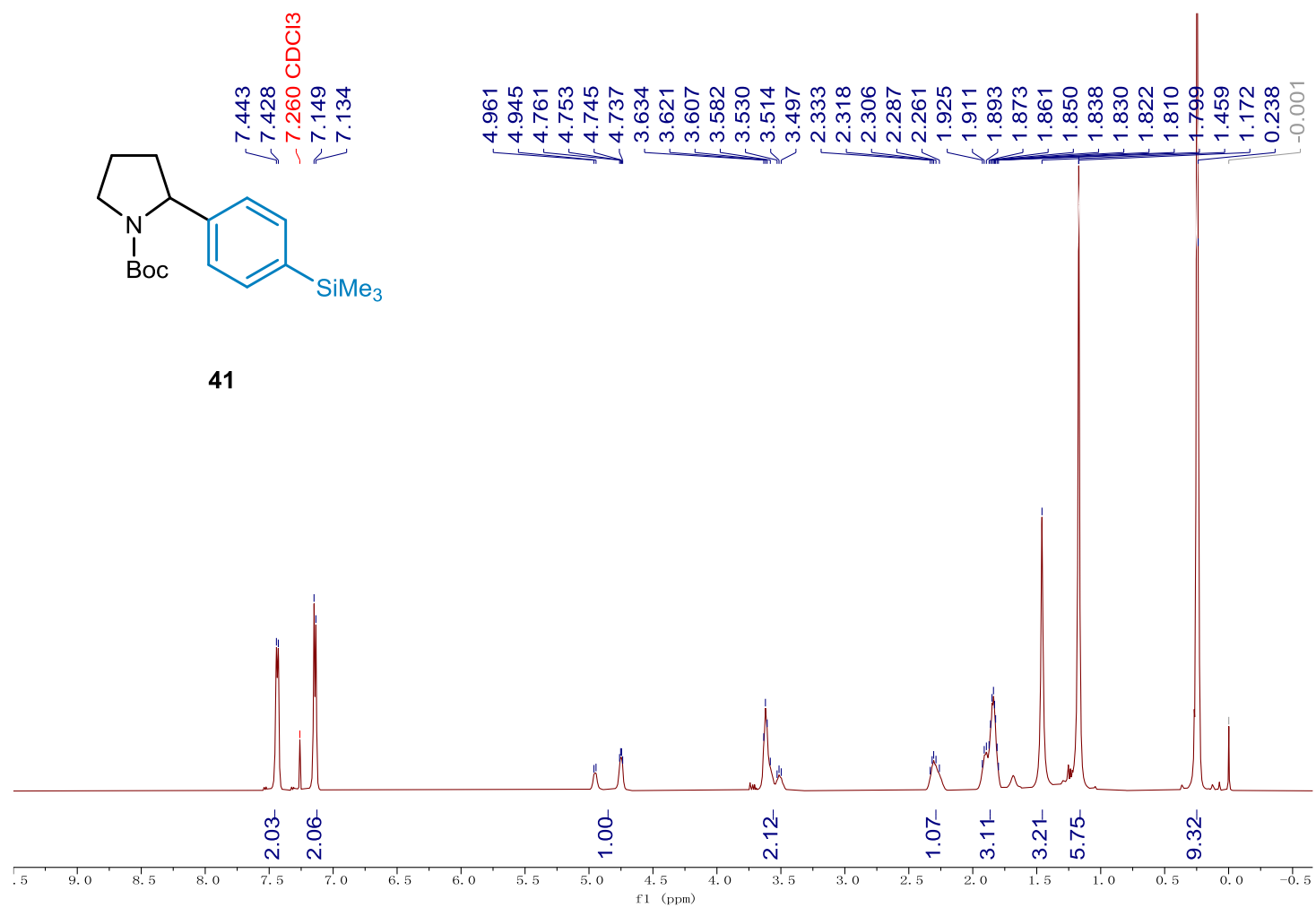

${ }^{13} \mathrm{C} \mathrm{NMR} 125 \mathrm{MHz}, \mathrm{CDCl}_{3}$

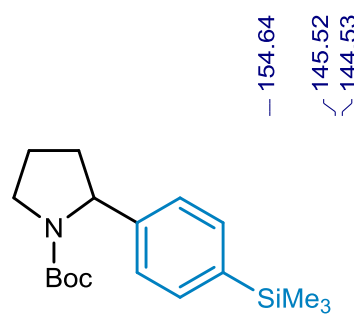

41

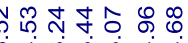

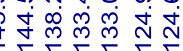

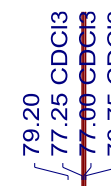

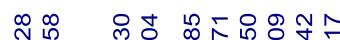

ঢ, 


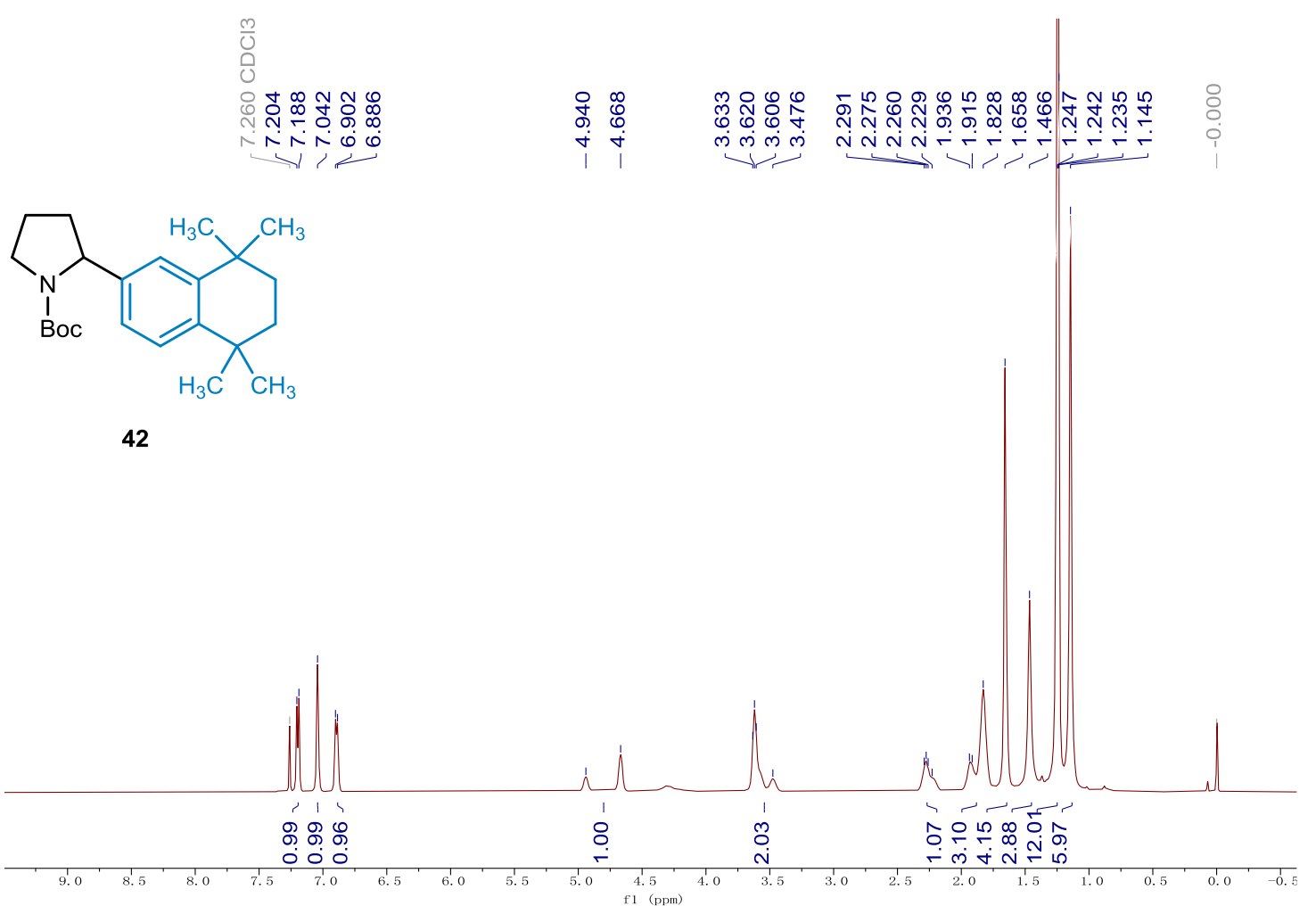

${ }^{13} \mathrm{C}$ NMR $125 \mathrm{MHz}, \mathrm{CDCl}_{3}$
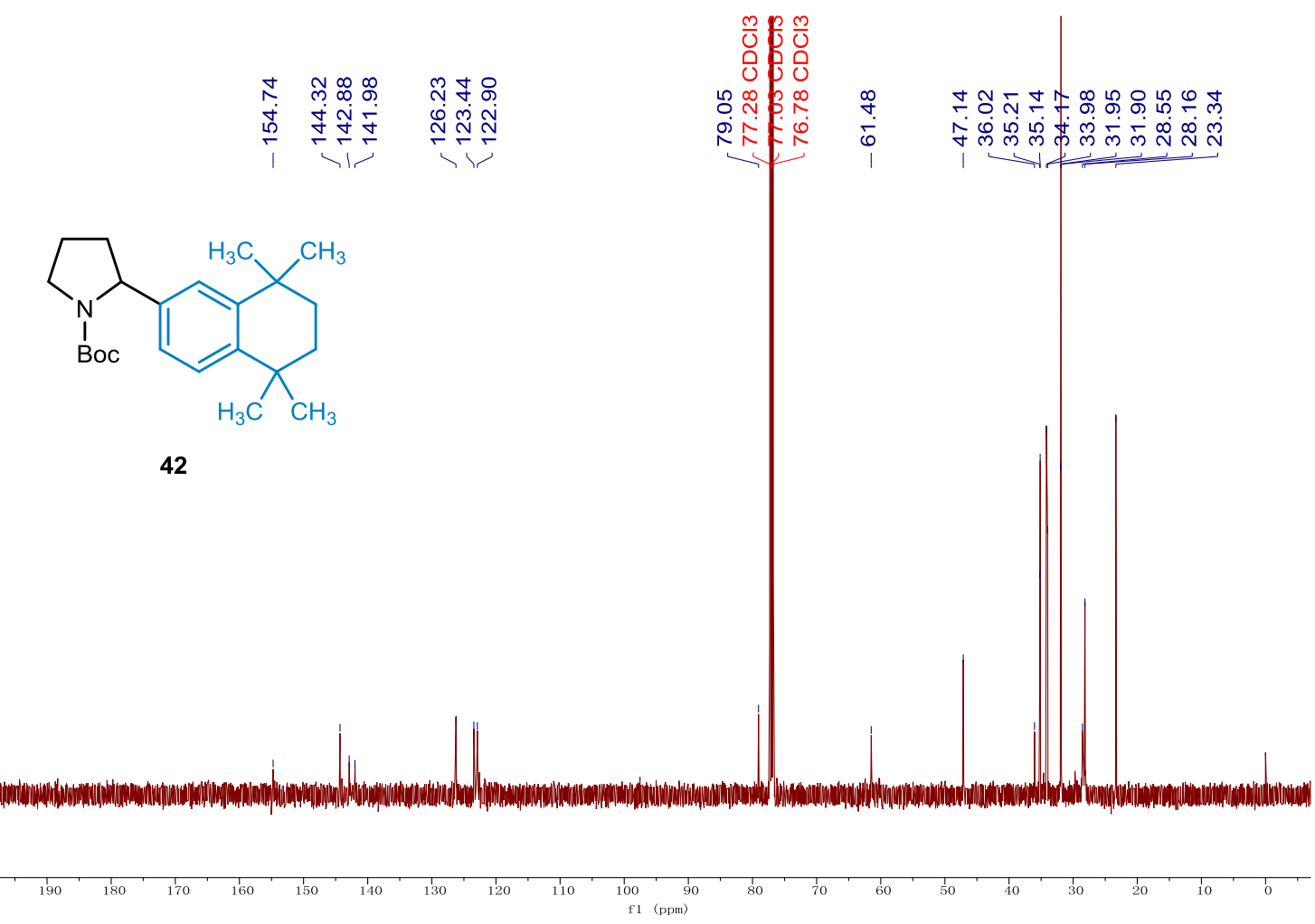

${ }^{1} \mathrm{H}$ NMR $500 \mathrm{MHz}, \mathrm{CDCl}_{3}$ 


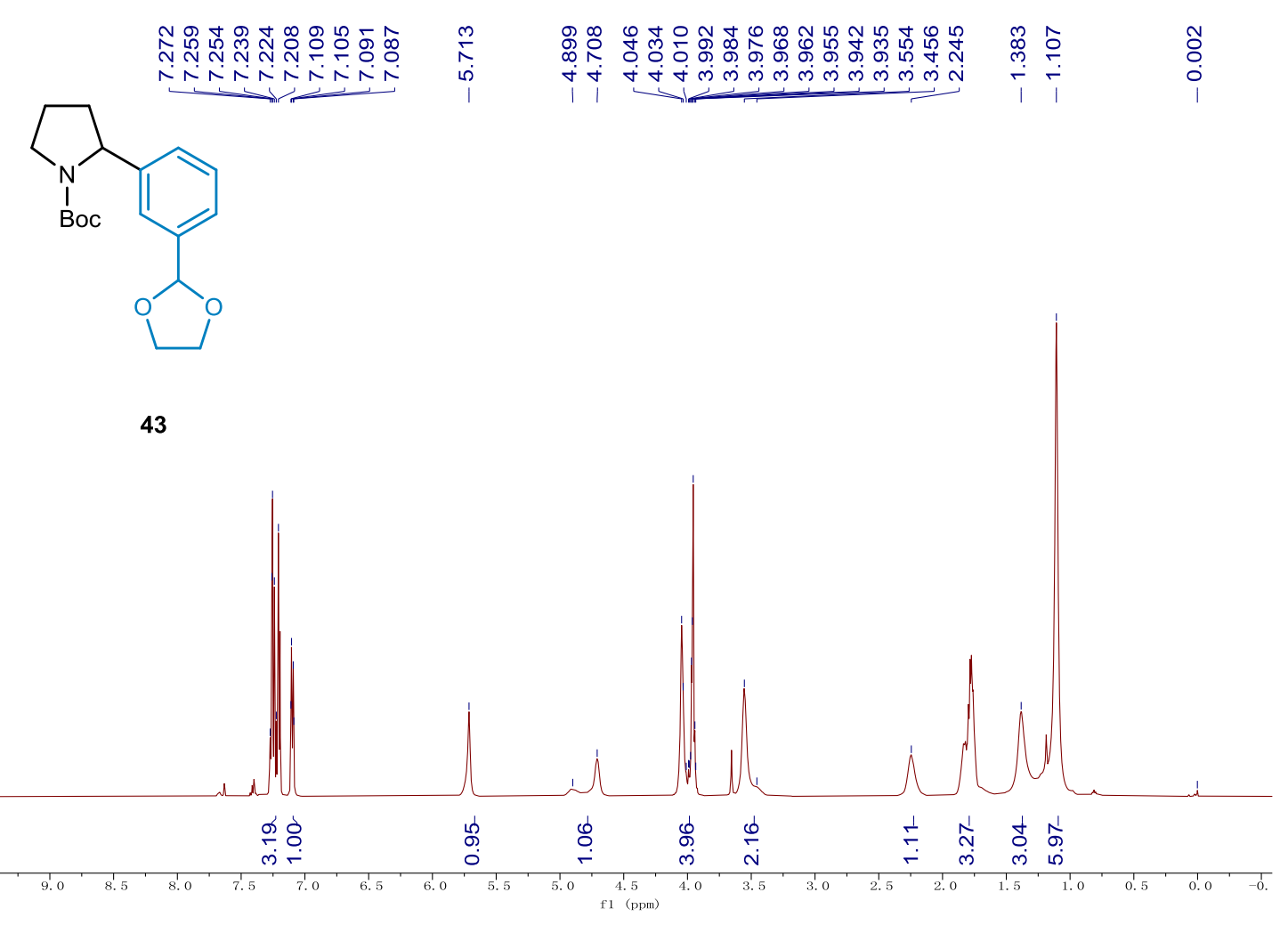

${ }^{13} \mathrm{C}$ NMR $125 \mathrm{MHz}, \mathrm{CDCl}_{3}$
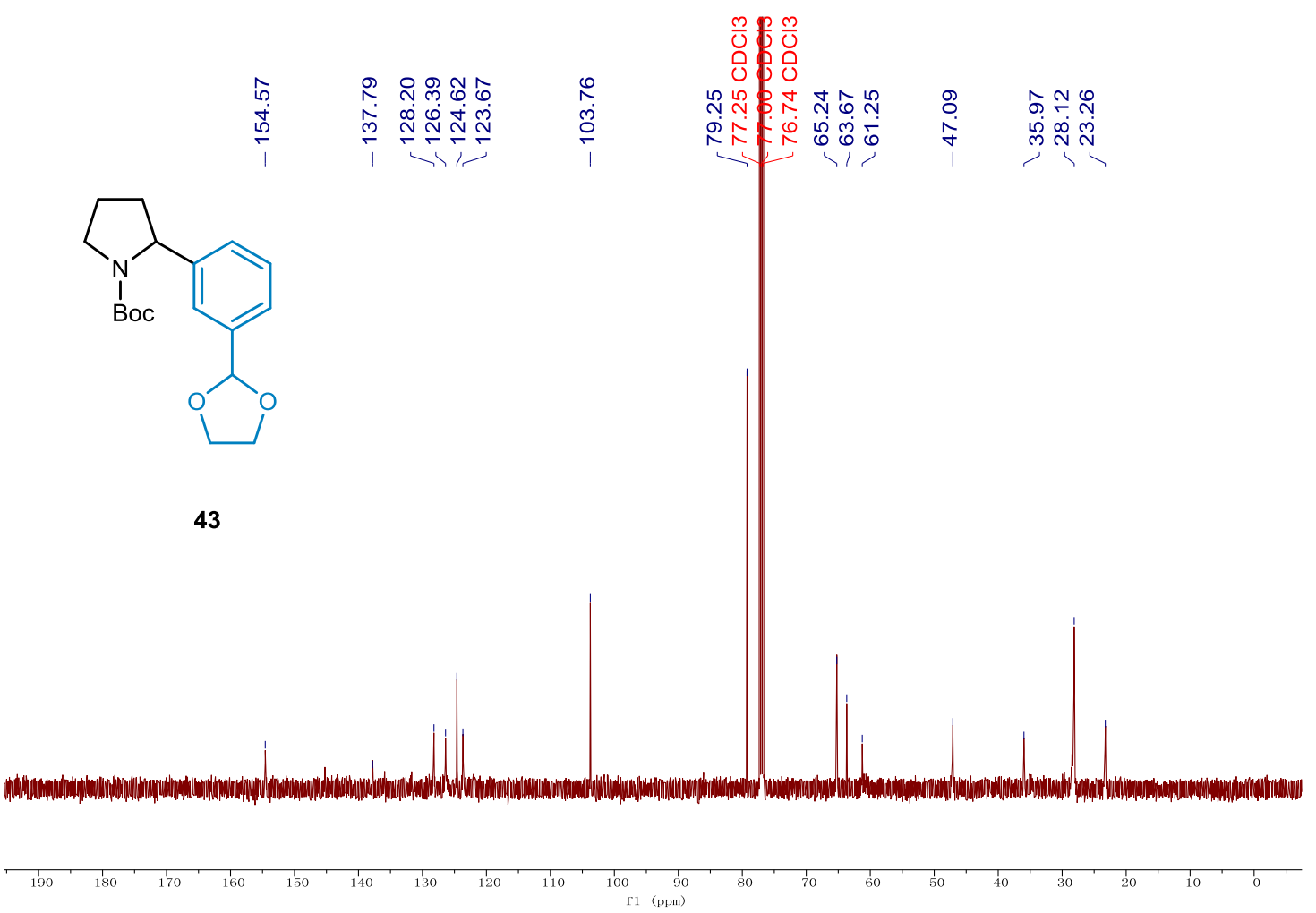

${ }^{1} \mathrm{H}$ NMR $500 \mathrm{MHz}, \mathrm{CDCl}_{3}$ 


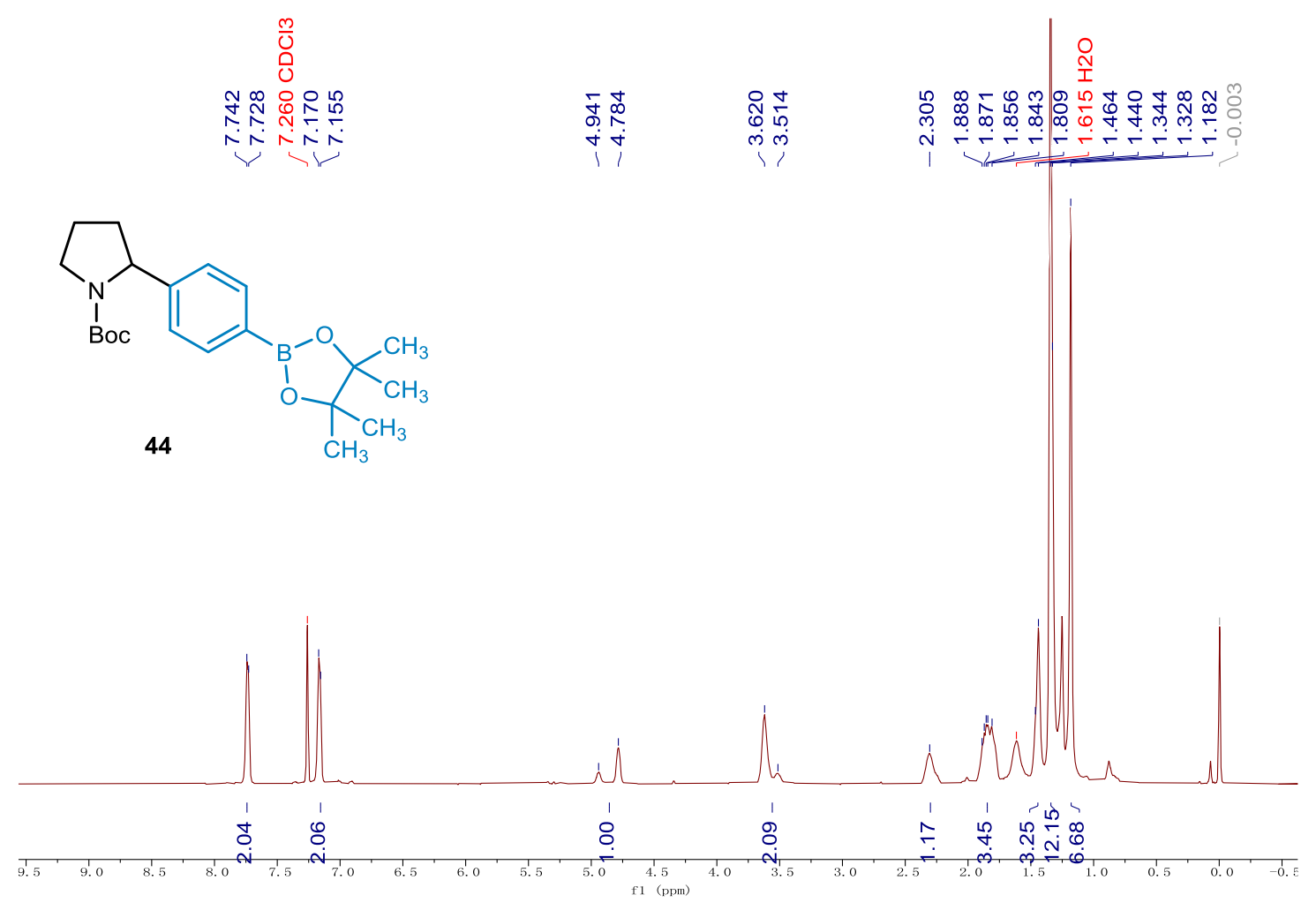

${ }^{13} \mathrm{C}$ NMR $125 \mathrm{MHz}, \mathrm{CDCl}_{3}$
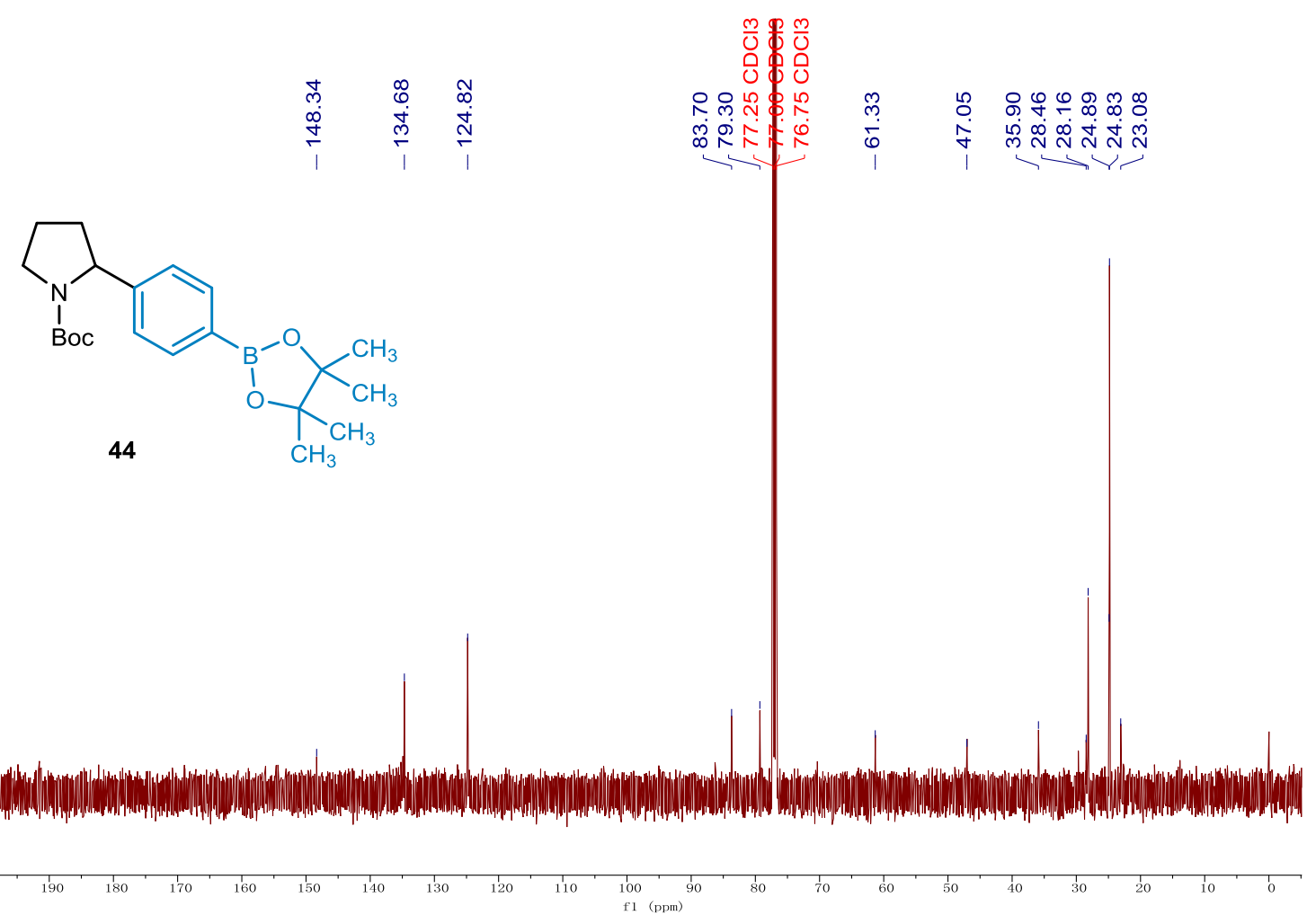

${ }^{1} \mathrm{H}$ NMR $500 \mathrm{MHz}, \mathrm{CDCl}_{3}$ 


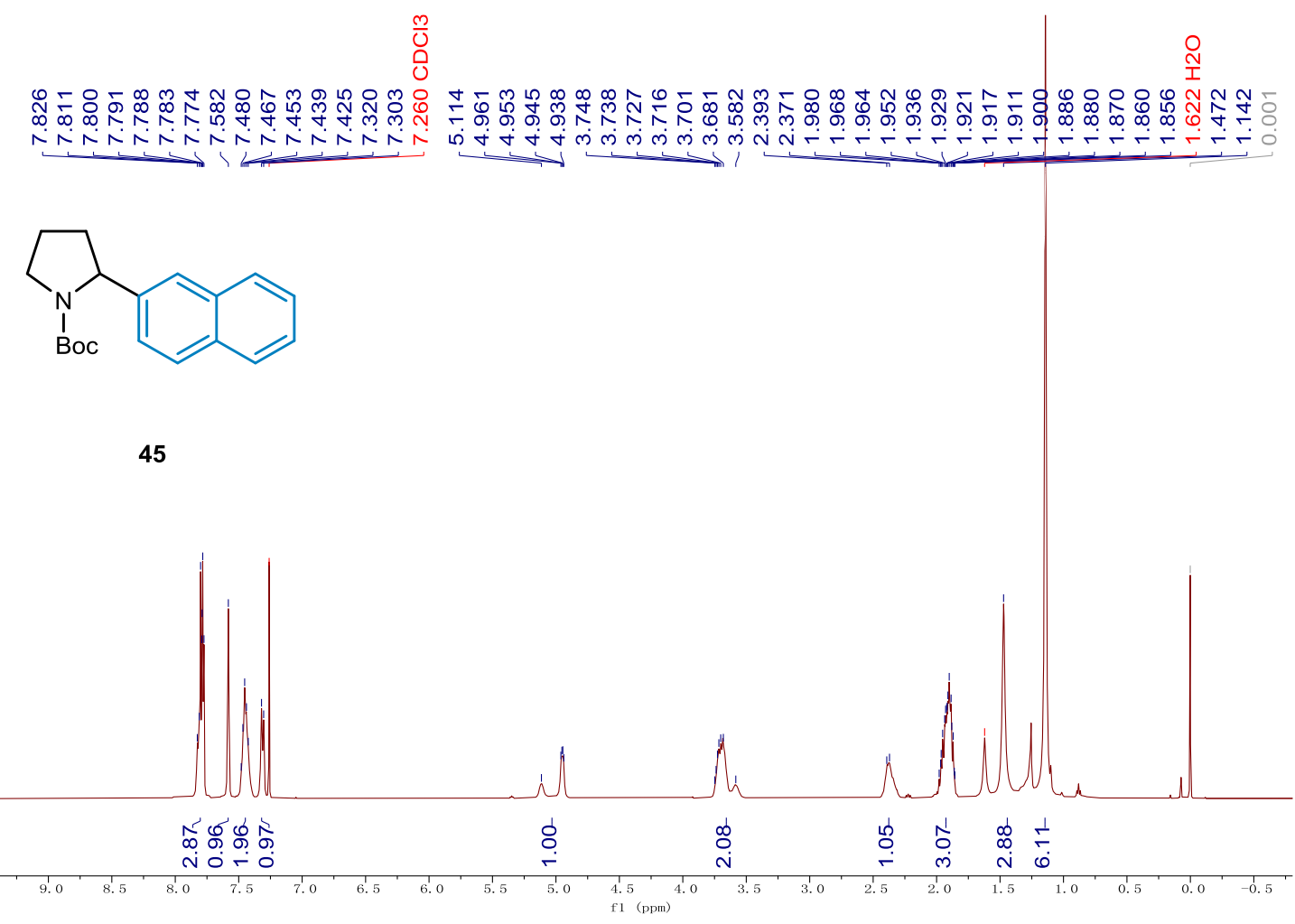

${ }^{13} \mathrm{C}$ NMR $125 \mathrm{MHz}, \mathrm{CDCl}_{3}$

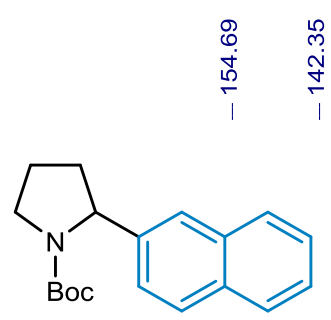

${ }^{1} \mathrm{H}$ NMR $500 \mathrm{MHz}, \mathrm{CDCl}_{3}$ 


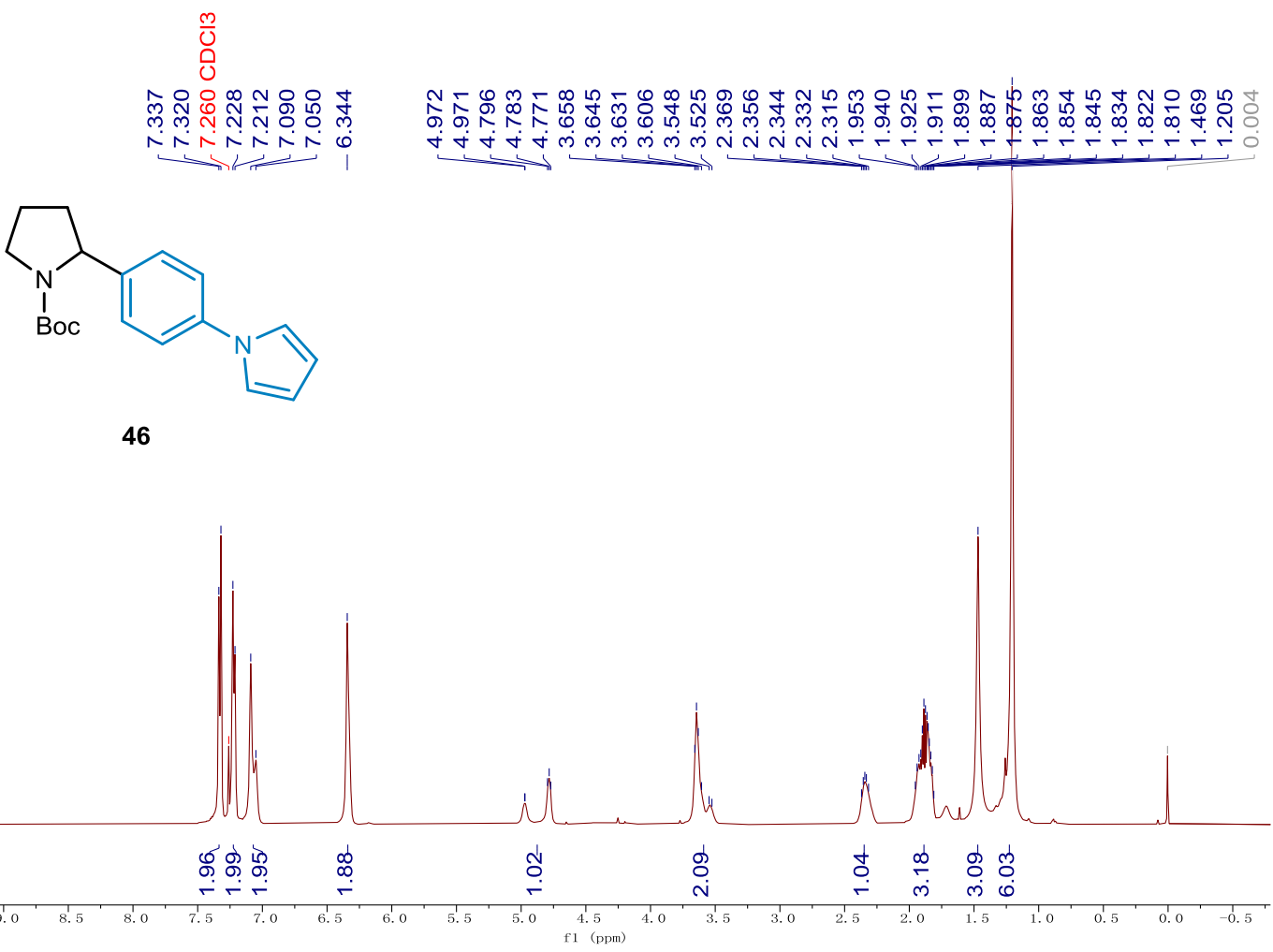

${ }^{13} \mathrm{C} \mathrm{NMR} 125 \mathrm{MHz}, \mathrm{CDCl}_{3}$

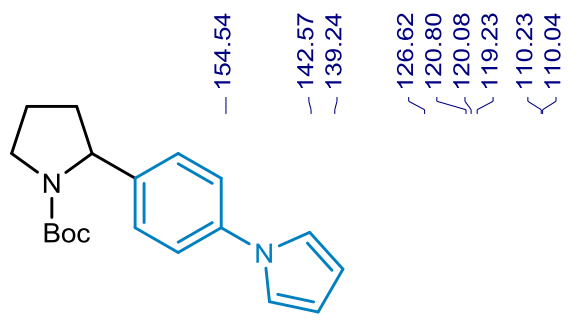

46
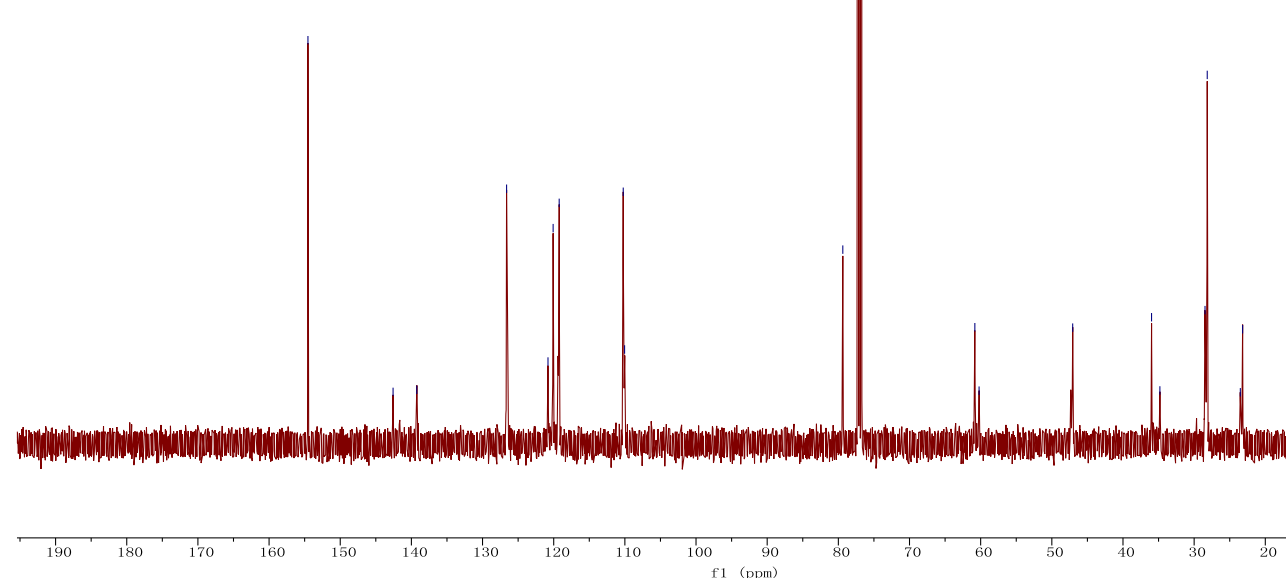

${ }^{1} \mathrm{H}$ NMR $500 \mathrm{MHz}, \mathrm{CDCl}_{3}$ 


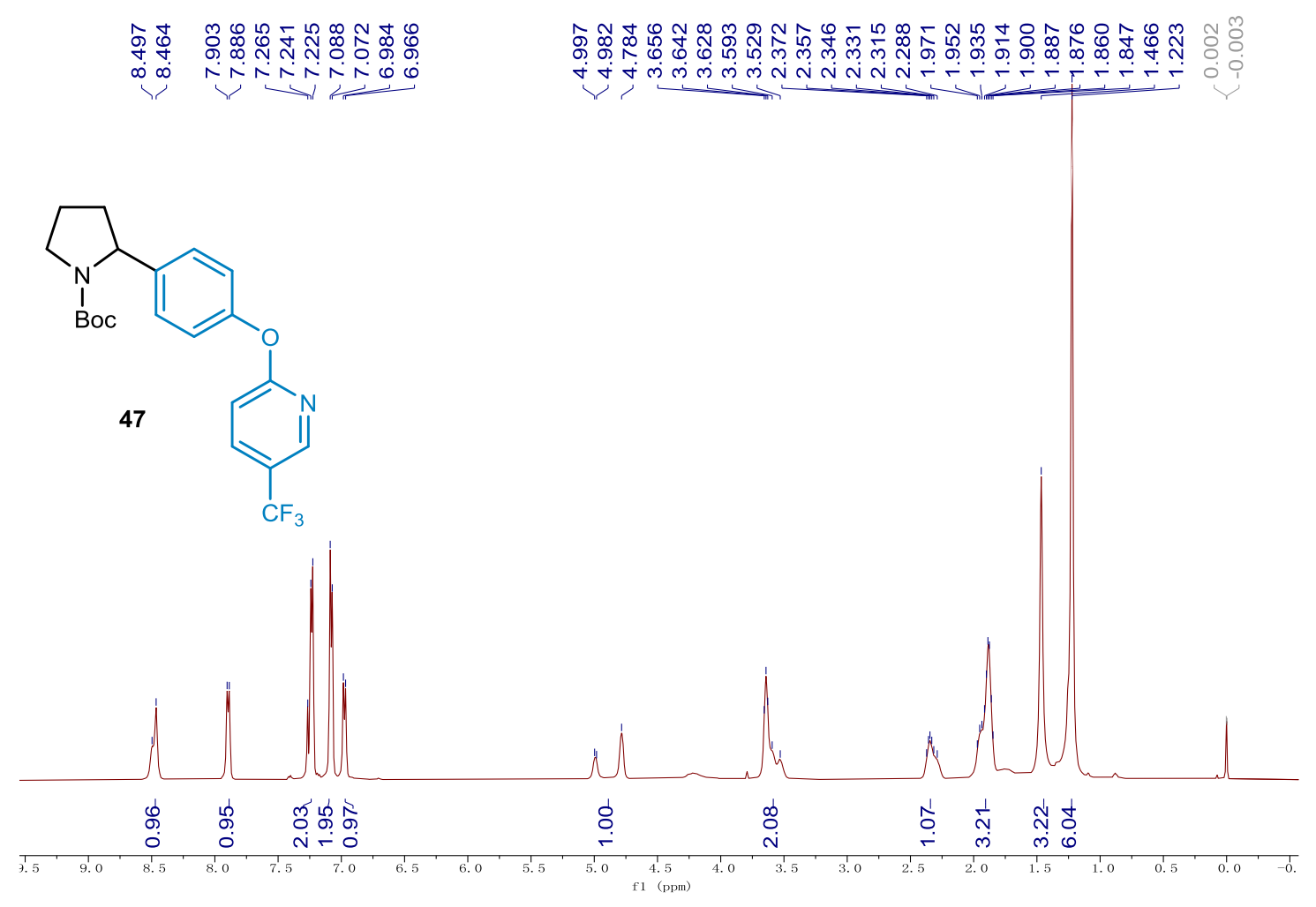

${ }^{13} \mathrm{C}$ NMR $125 \mathrm{MHz}, \mathrm{CDCl}_{3}$

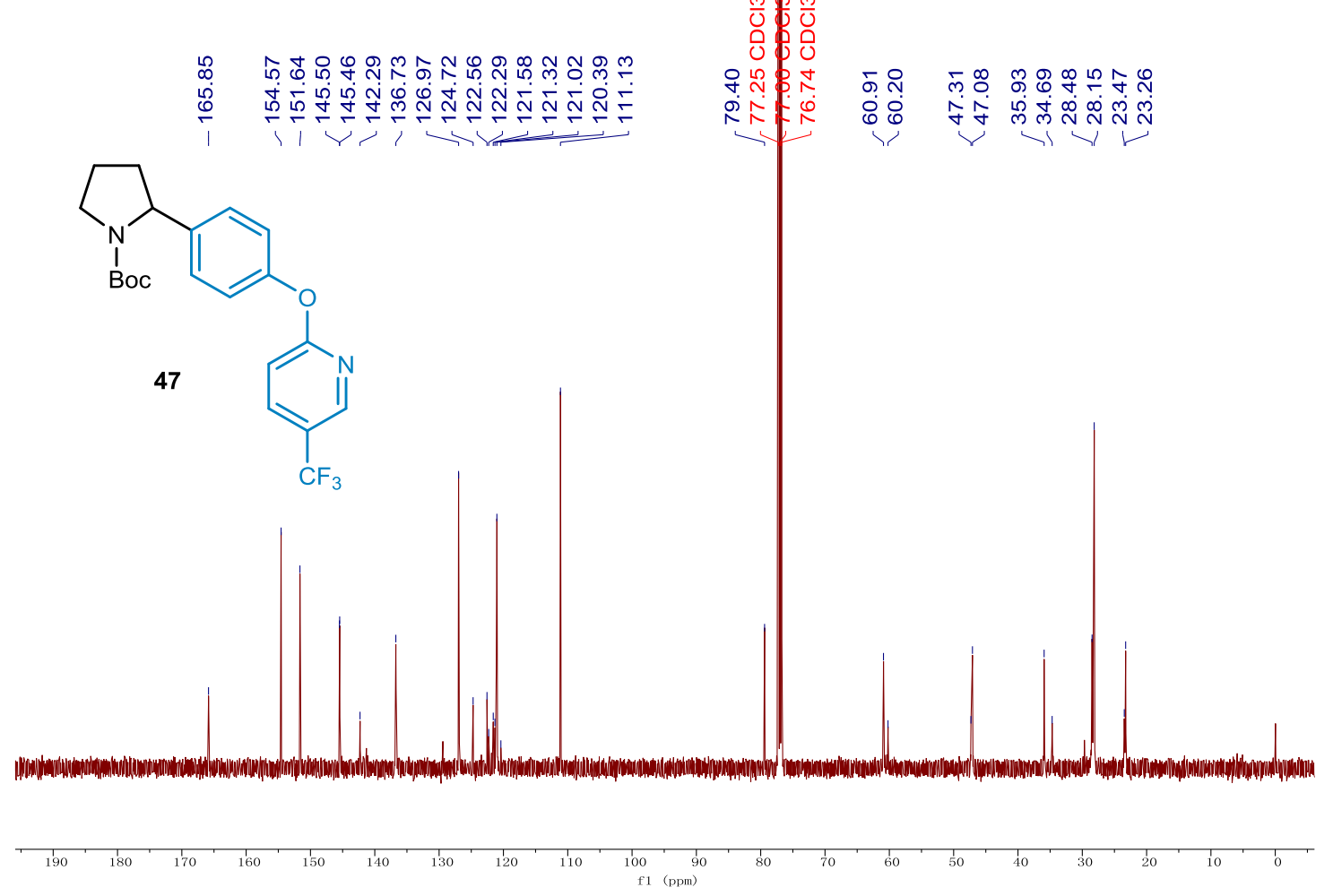

${ }^{19} \mathrm{~F}$ NMR $470 \mathrm{MHz}, \mathrm{CDCl}_{3}$ 


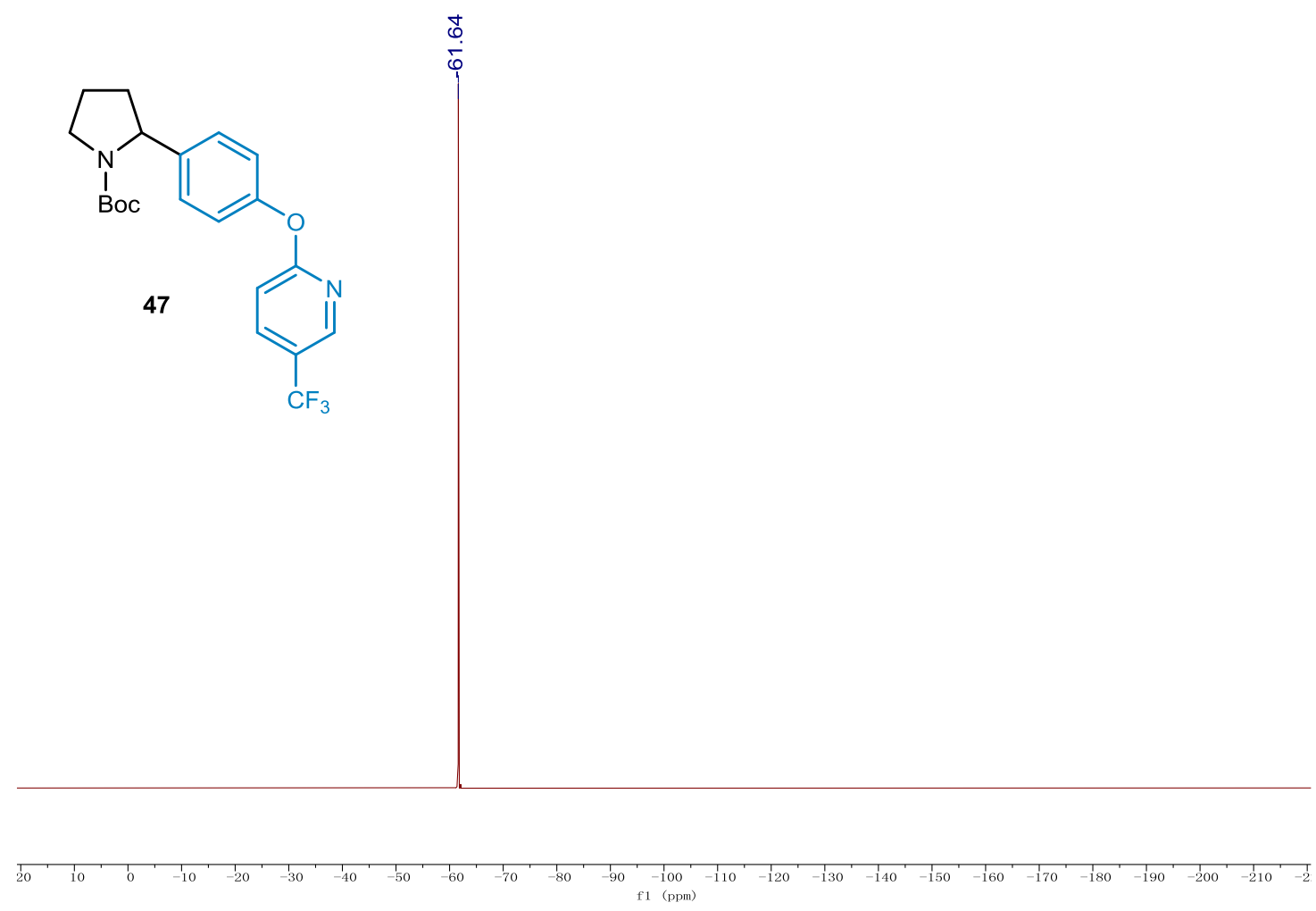

${ }^{1} \mathrm{H}$ NMR $500 \mathrm{MHz}, \mathrm{CDCl}_{3}$ 

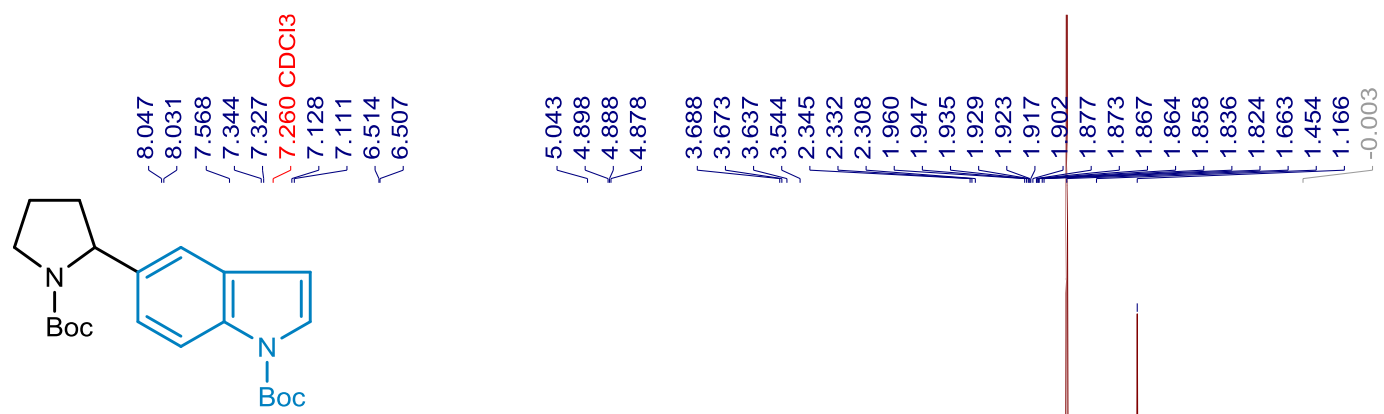

48

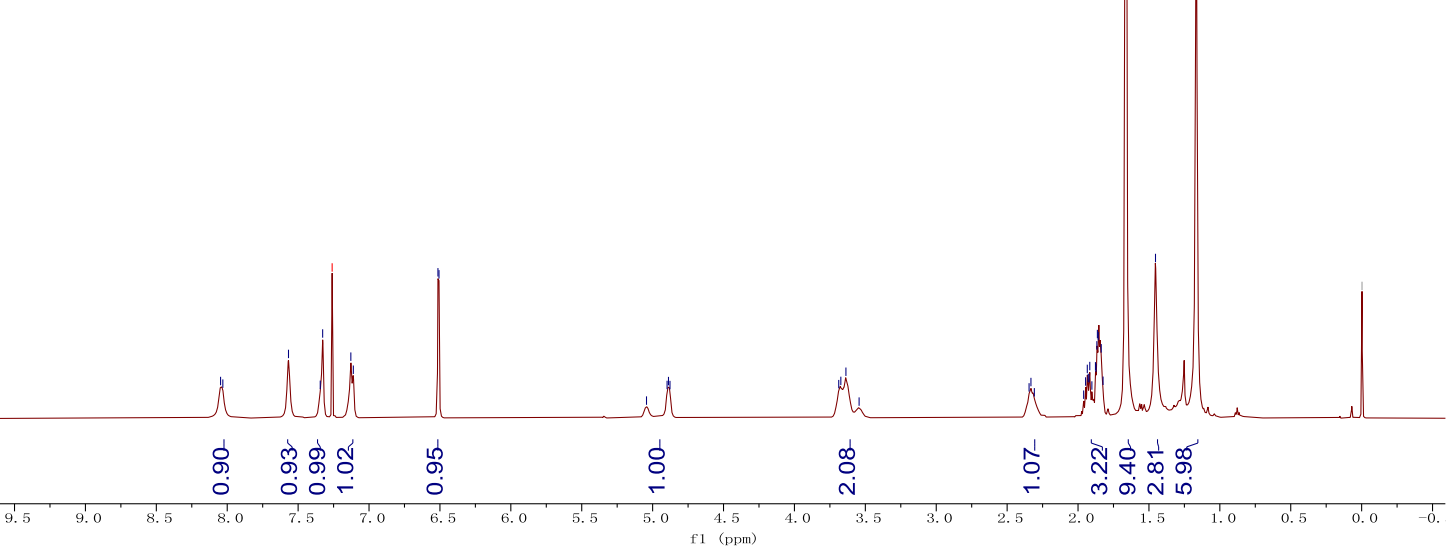

${ }^{13} \mathrm{C} \mathrm{NMR} 125 \mathrm{MHz}, \mathrm{CDCl}_{3}$

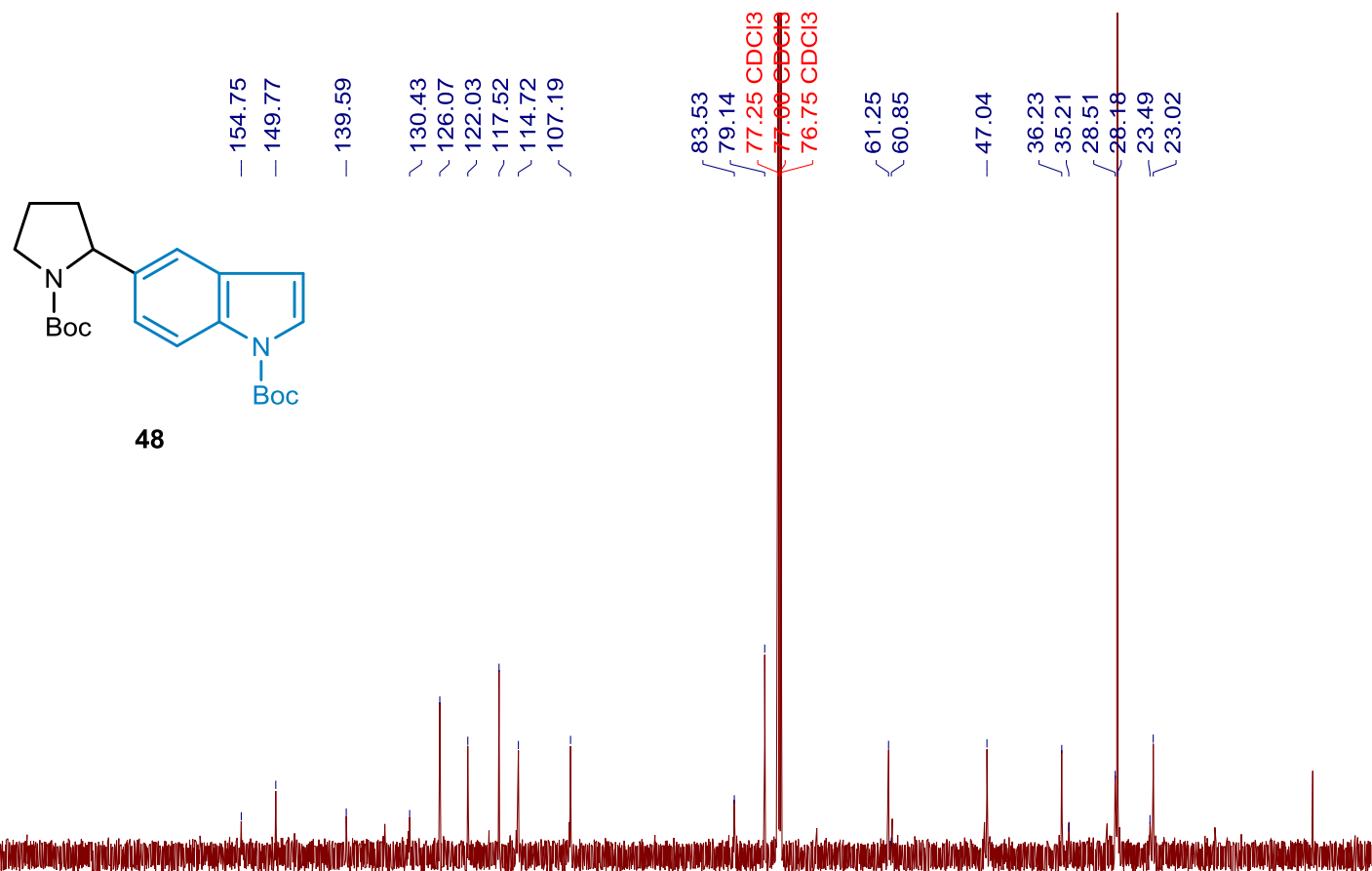

${ }^{1} \mathrm{H}$ NMR $500 \mathrm{MHz}, \mathrm{CDCl}_{3}$ 

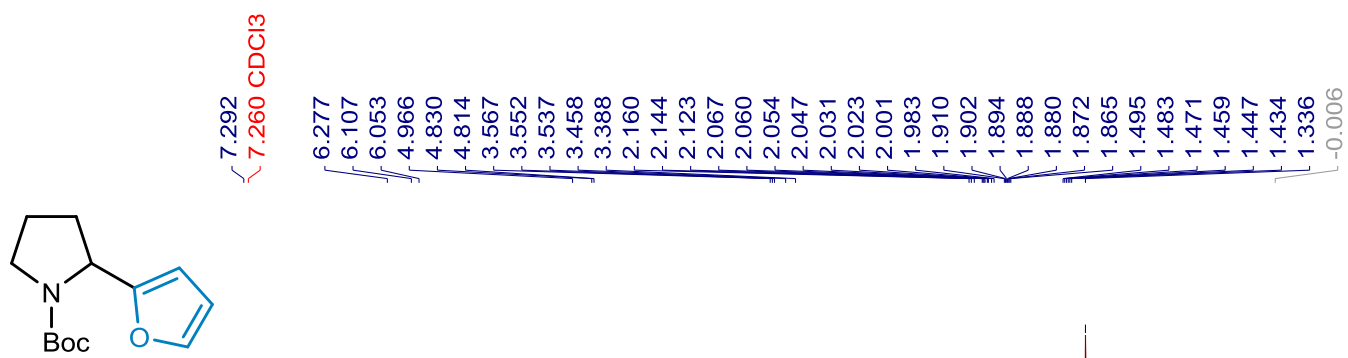

49

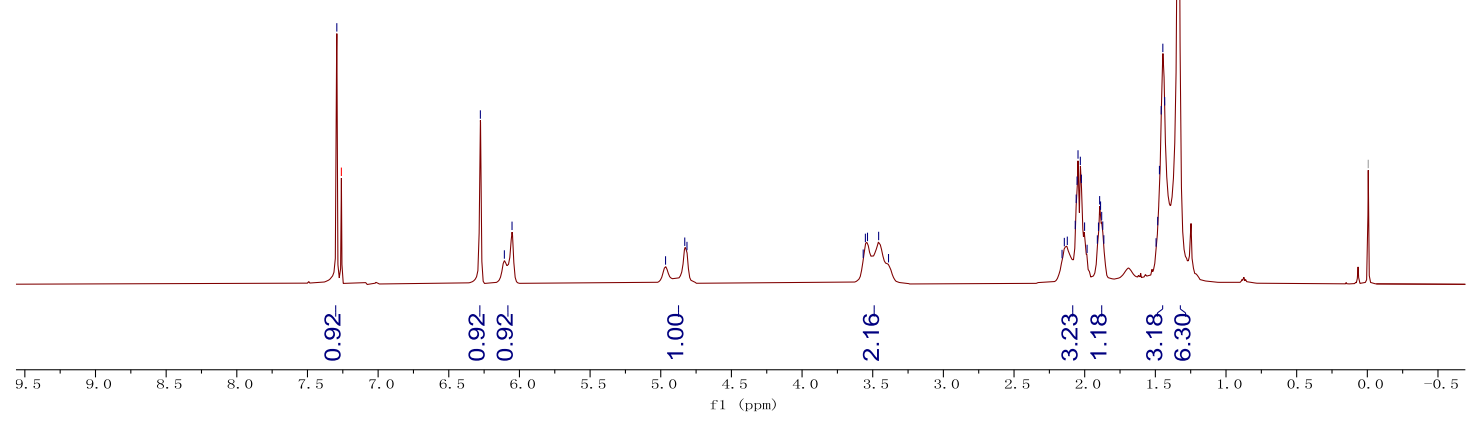

${ }^{13} \mathrm{C}$ NMR $125 \mathrm{MHz}, \mathrm{CDCl}_{3}$
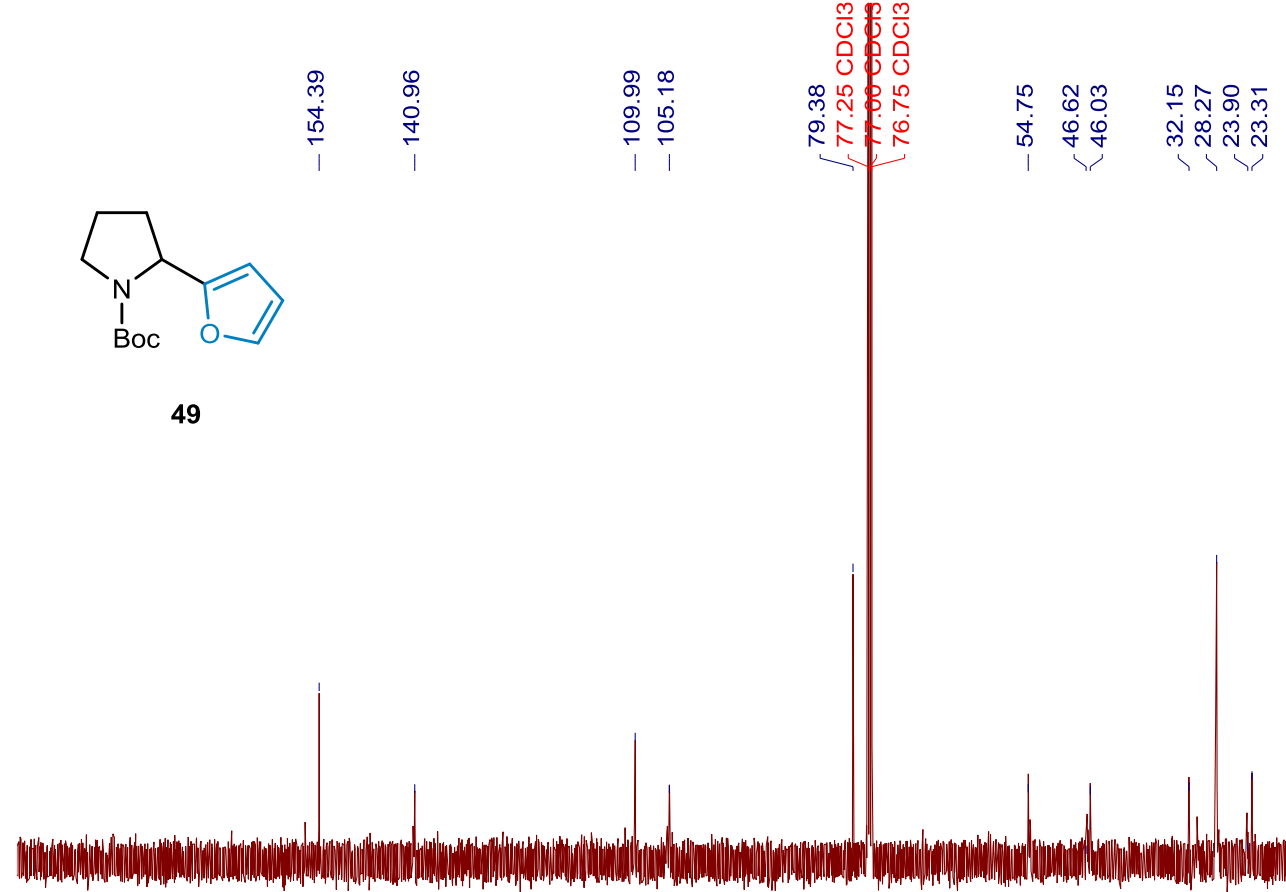

${ }^{1} \mathrm{H}$ NMR $400 \mathrm{MHz}, \mathrm{CDCl}_{3}$ 


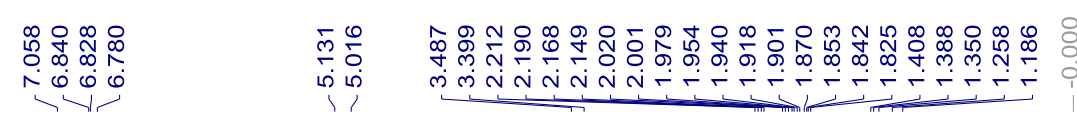<smiles>O=C(c1ccccc1)N1CCCC1c1cccs1</smiles>

50

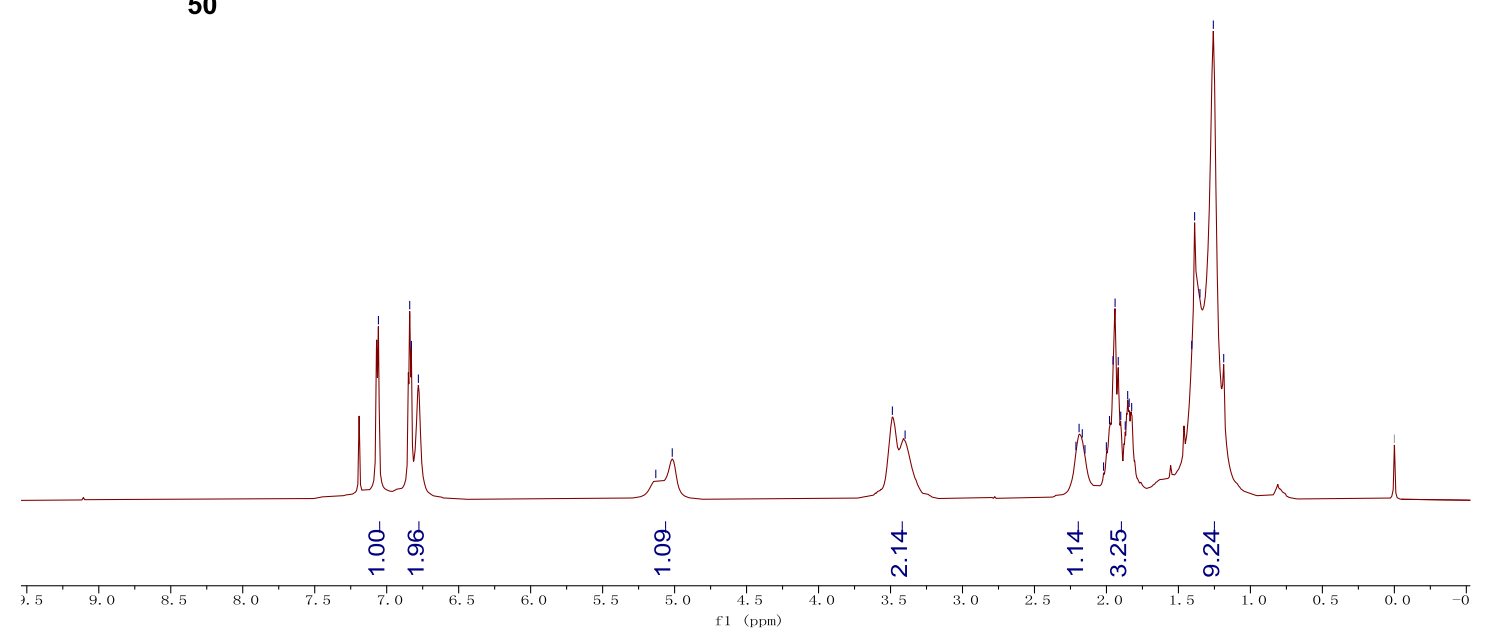

${ }^{13} \mathrm{C} \mathrm{NMR} 100 \mathrm{MHz}, \mathrm{CDCl}_{3}$

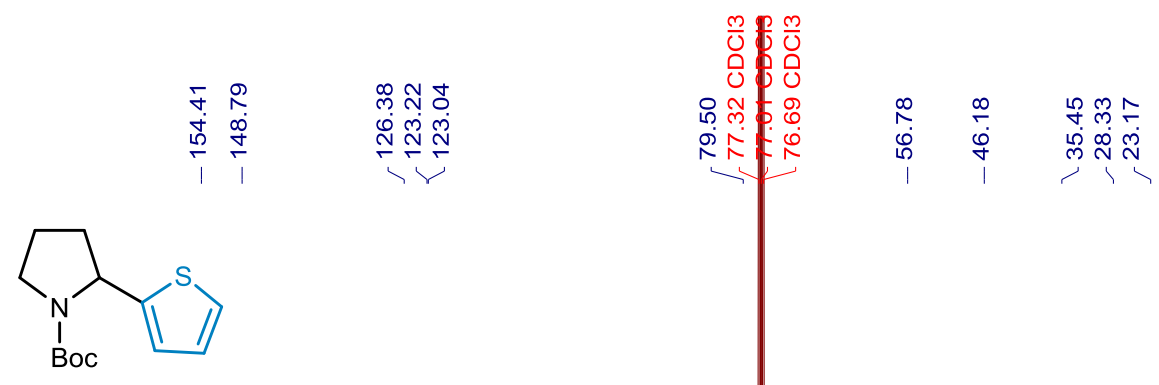

50
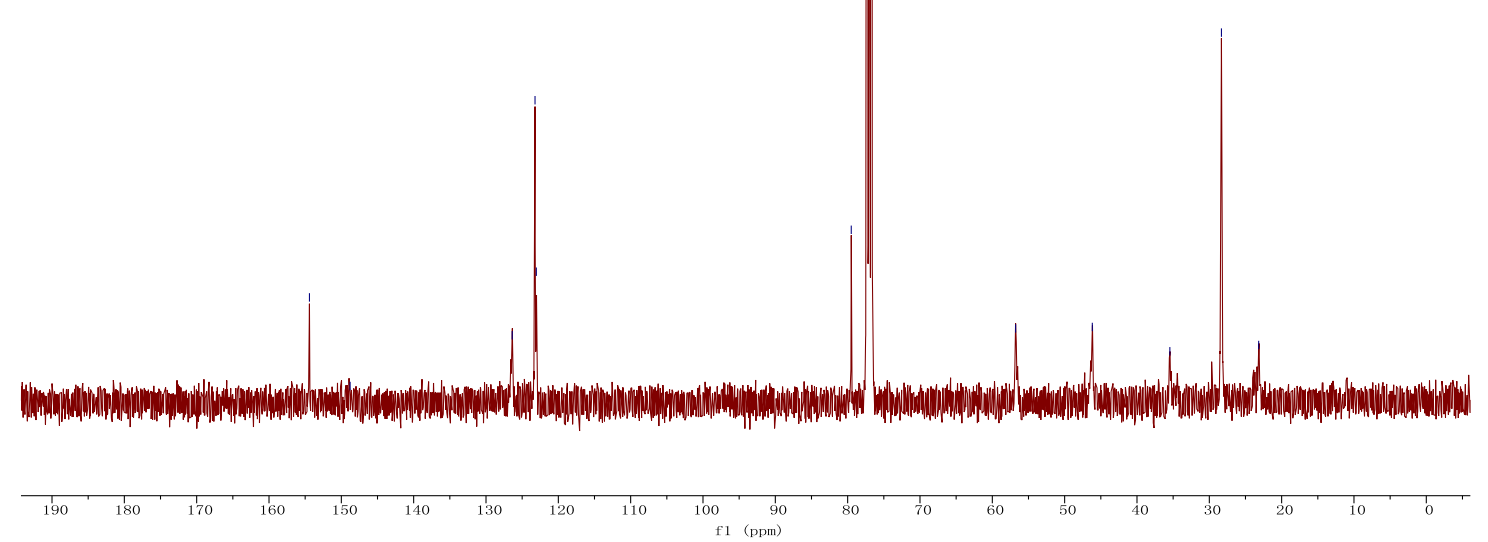

${ }^{1} \mathrm{H}$ NMR $500 \mathrm{MHz}, \mathrm{CDCl}_{3}$ 


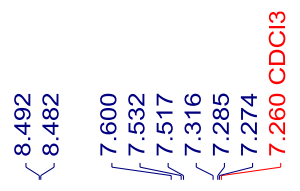

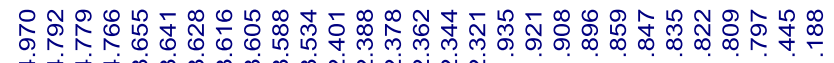

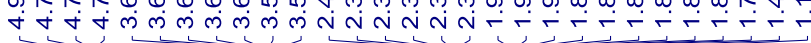<smiles>CC(C)(C)OC(=O)N1CCCC1c1cccnc1</smiles>

51

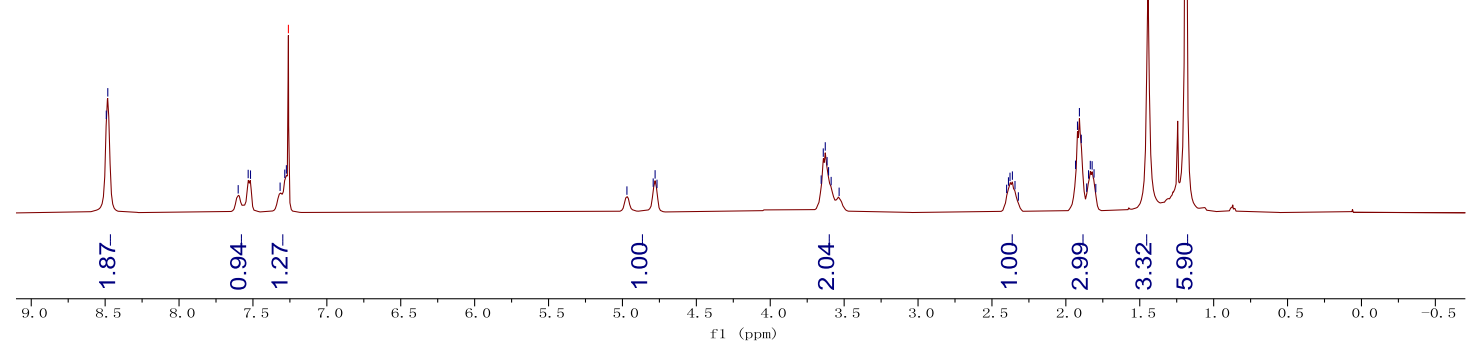

${ }^{13} \mathrm{C}$ NMR $125 \mathrm{MHz}, \mathrm{CDCl}_{3}$

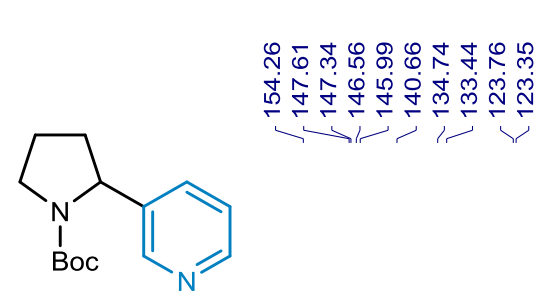

51

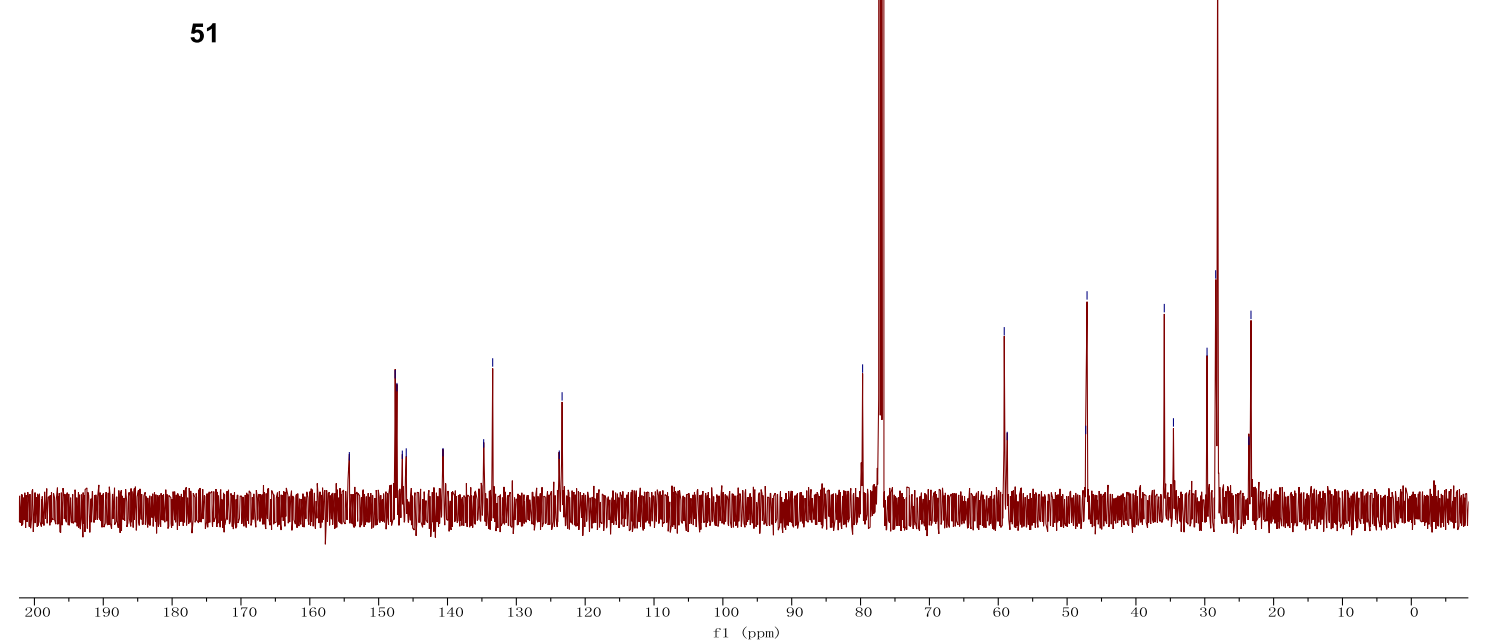

${ }^{1} \mathrm{H}$ NMR $500 \mathrm{MHz}, \mathrm{CDCl}_{3}$ 


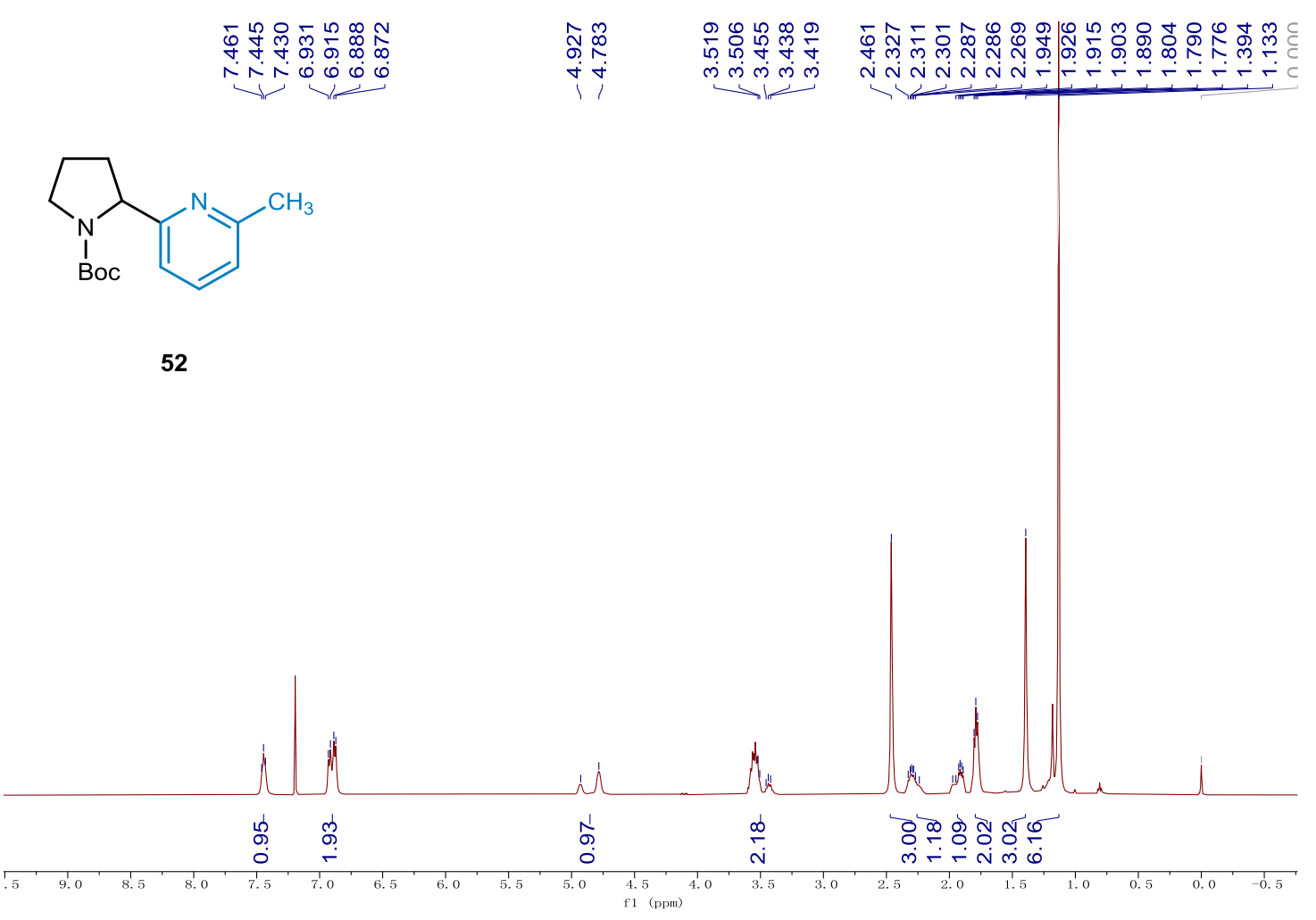

${ }^{13} \mathrm{C}$ NMR $125 \mathrm{MHz}, \mathrm{CDCl}_{3}$

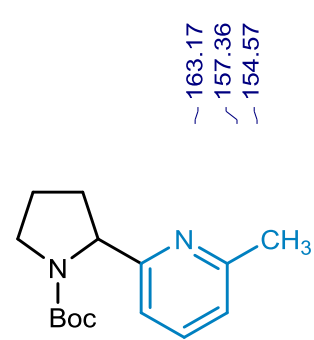

52

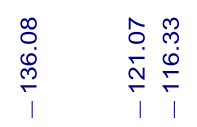

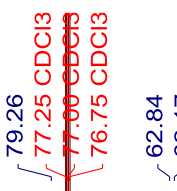

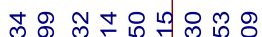

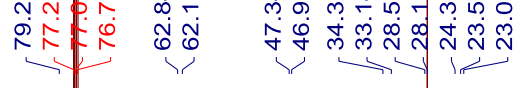

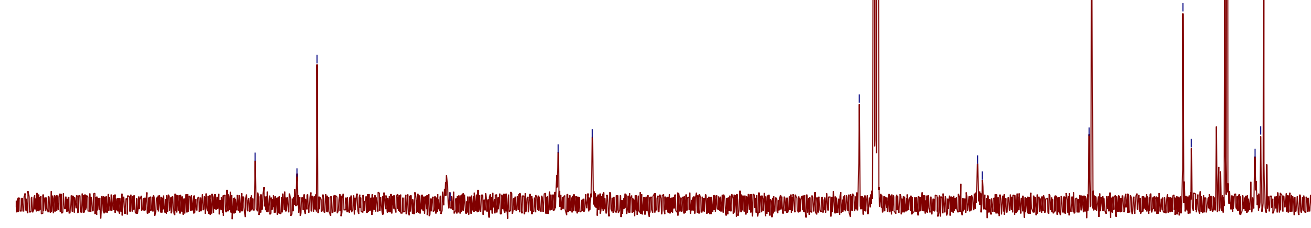

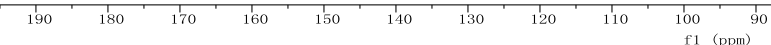

${ }^{1} \mathrm{H}$ NMR $500 \mathrm{MHz}, \mathrm{CDCl}_{3}$ 


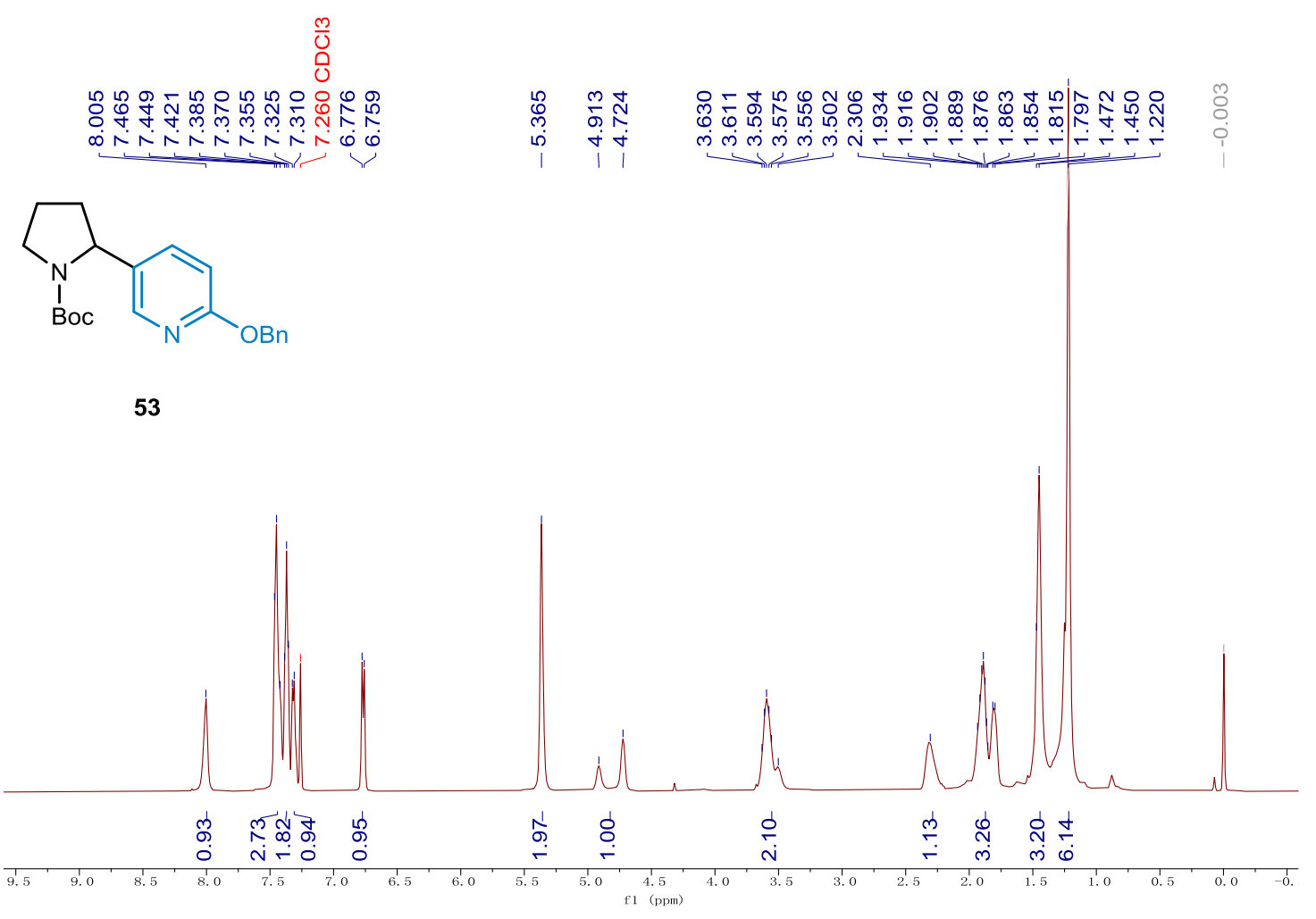

${ }^{13} \mathrm{C} \mathrm{NMR} 125 \mathrm{MHz}, \mathrm{CDCl}_{3}$

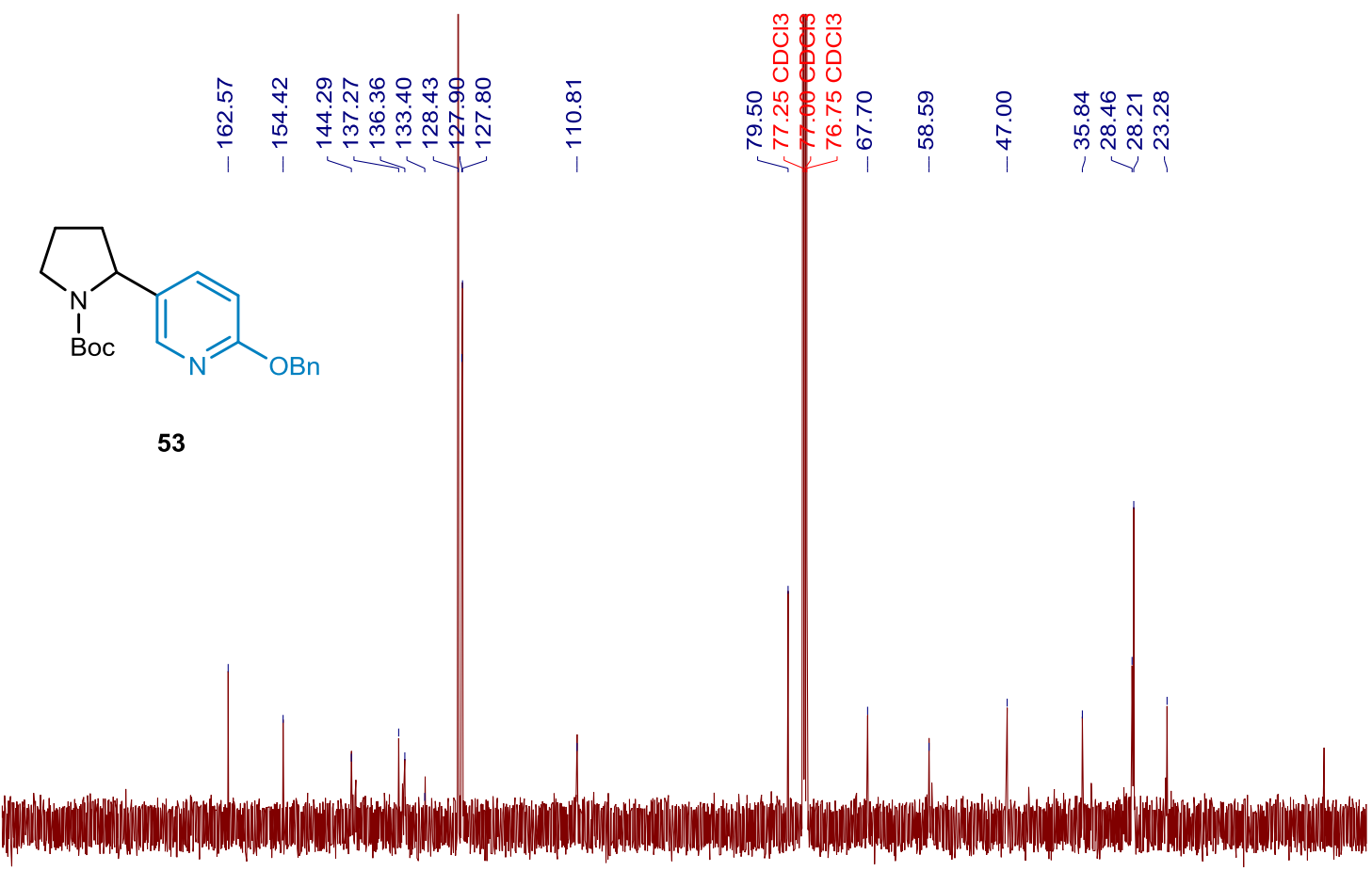

${ }^{1} \mathrm{H}$ NMR $500 \mathrm{MHz}, \mathrm{CDCl}_{3}$ 


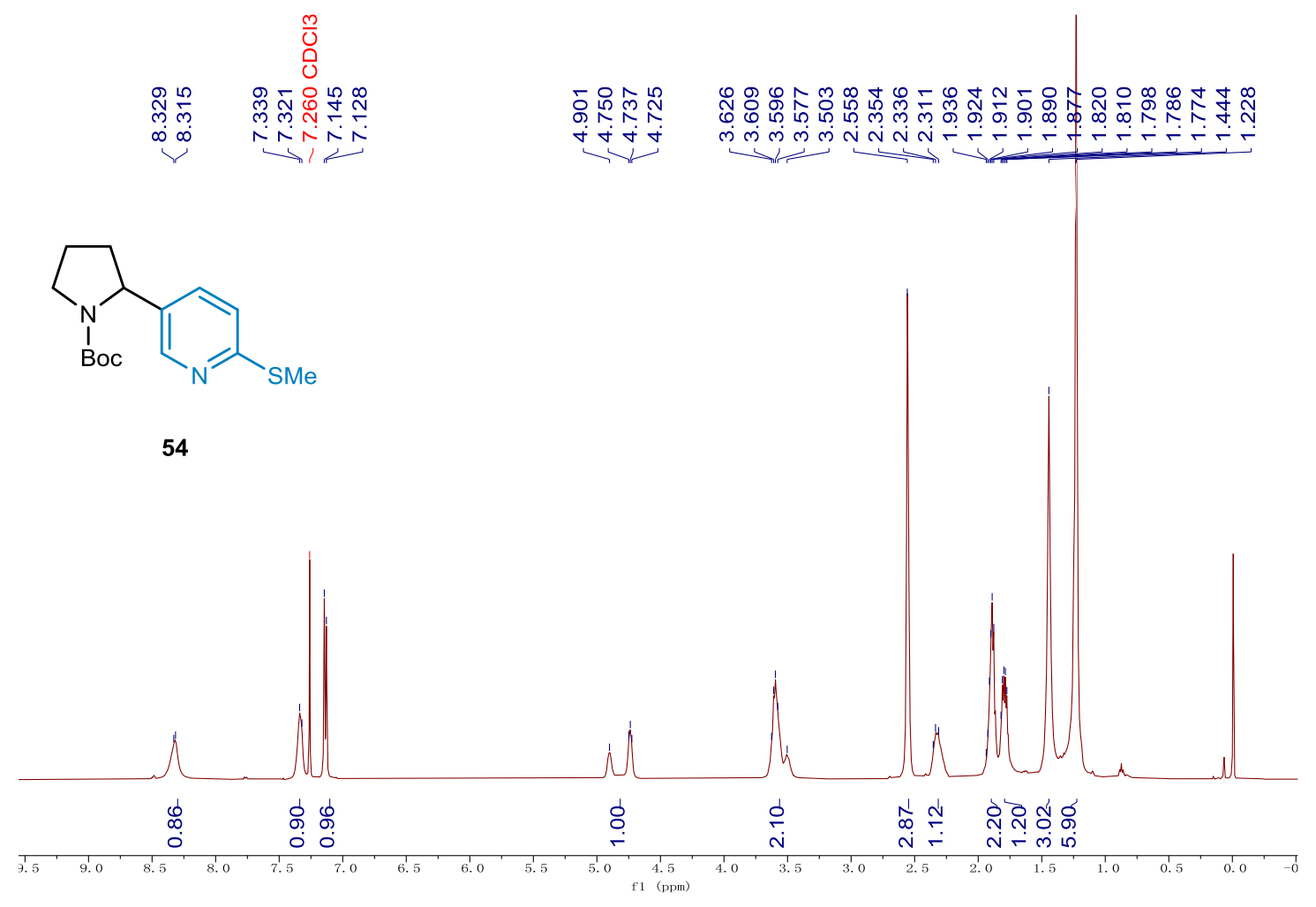

${ }^{13} \mathrm{C}$ NMR $125 \mathrm{MHz}, \mathrm{CDCl}_{3}$

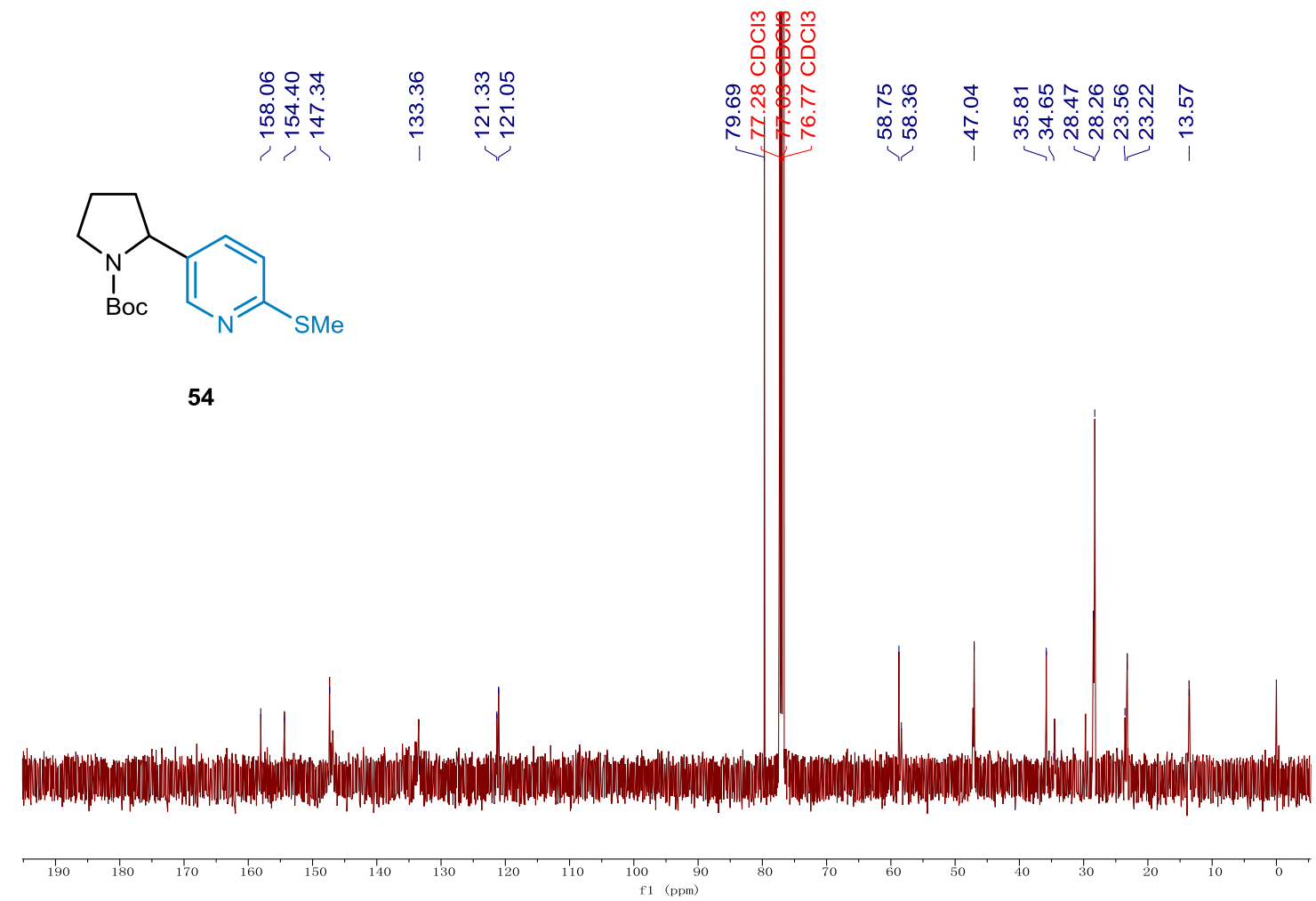

${ }^{1} \mathrm{H}$ NMR $500 \mathrm{MHz}, \mathrm{CDCl}_{3}$ 
$\frac{m}{0}$

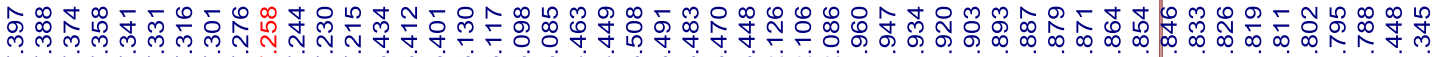

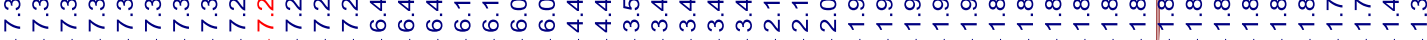<smiles>CC(C)(C)OC(=O)N1CCCC1/C=C\c1ccccc1</smiles>

$55, E / Z=4: 1$

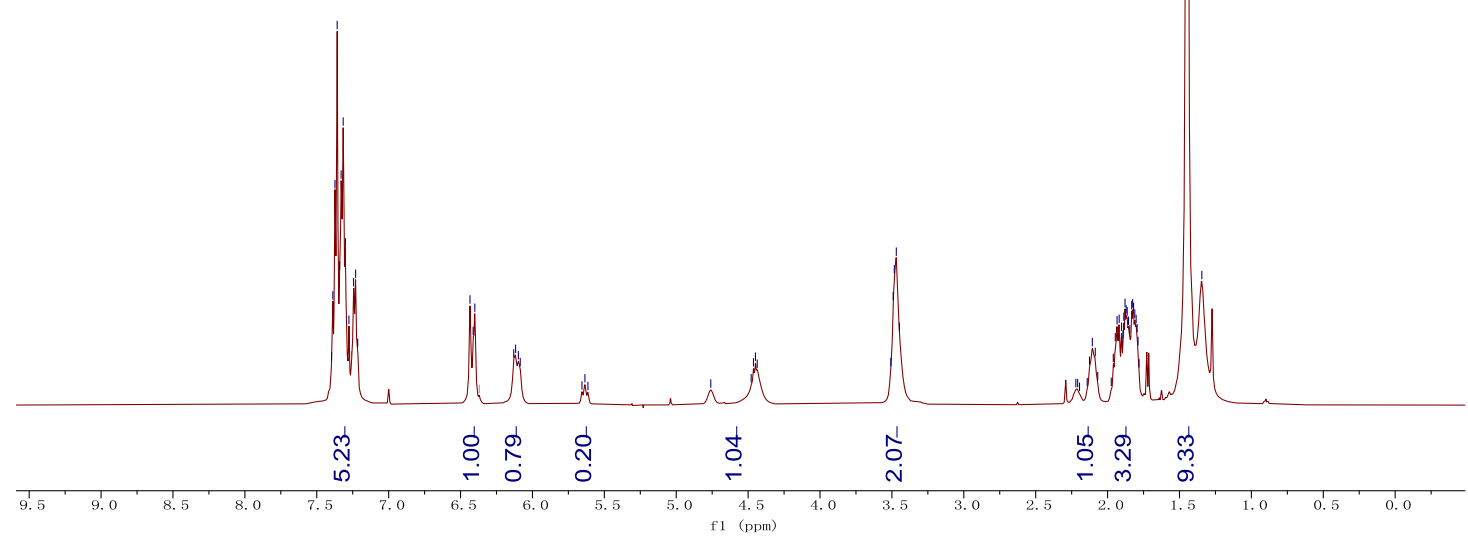

${ }^{1} \mathrm{H}$ NMR $400 \mathrm{MHz}, \mathrm{CDCl}_{3}$

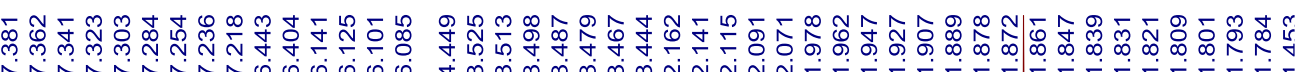

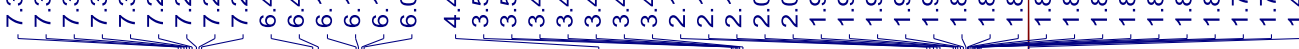<smiles>CC(C)(C)OC(=O)N1CCCC1/C=C/c1ccccc1</smiles>

E isomer

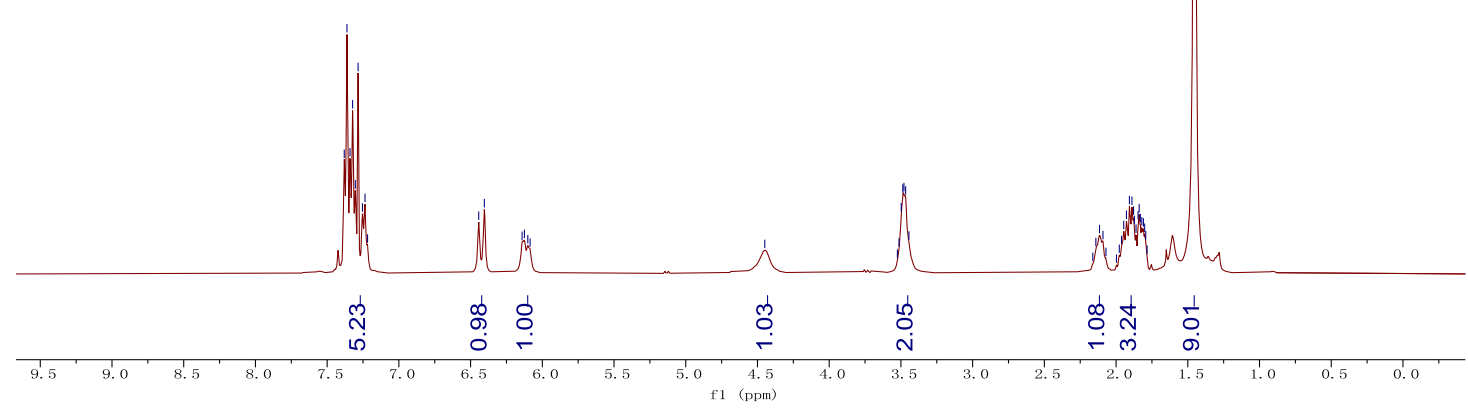

${ }^{13} \mathrm{C} \mathrm{NMR} 100 \mathrm{MHz}, \mathrm{CDCl}_{3}$ 

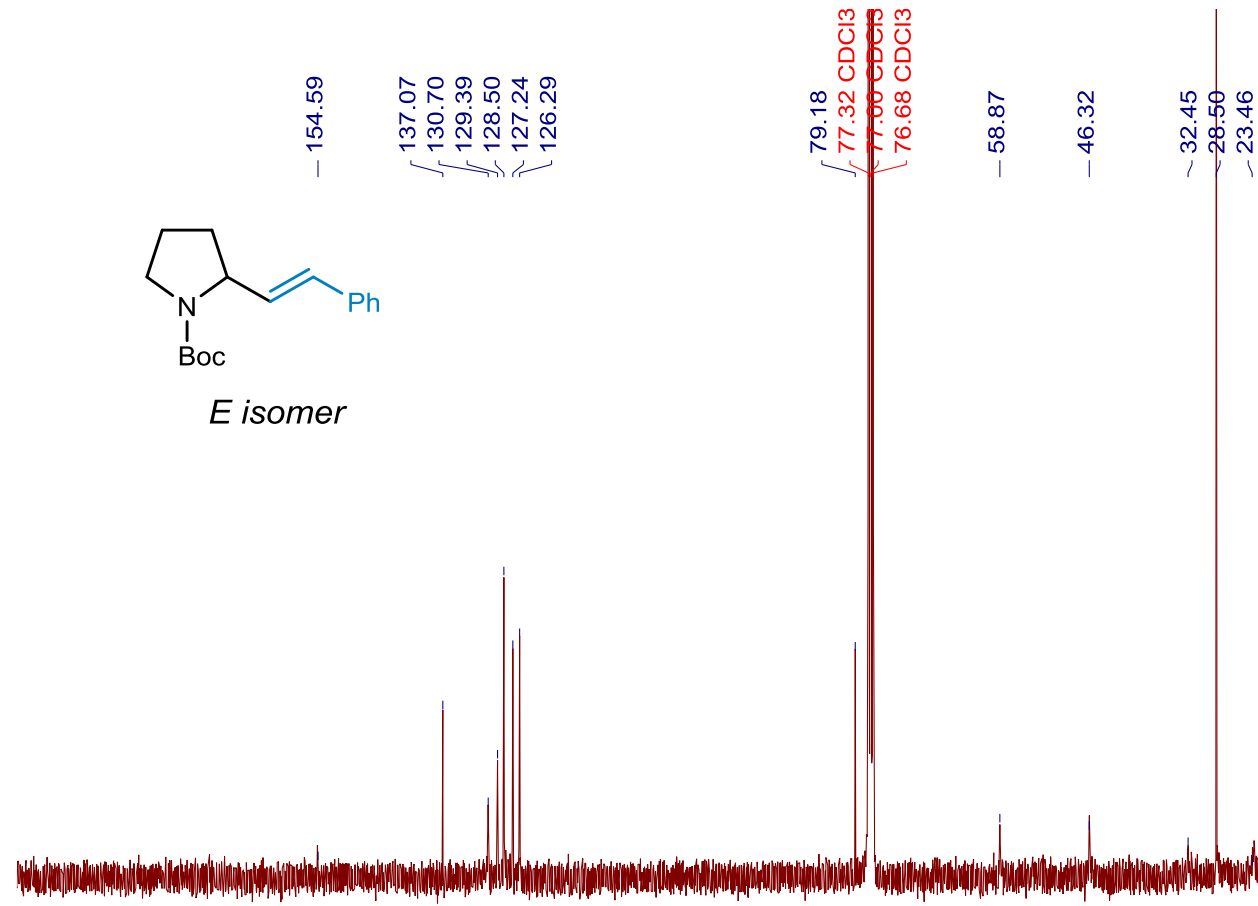

$E$ isomer

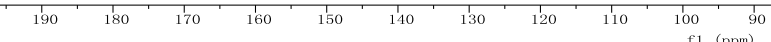




\section{XRD-characterization data}

X-Ray Crystal Structure of $\mathbf{5 6}$ (CCDC 2056187)

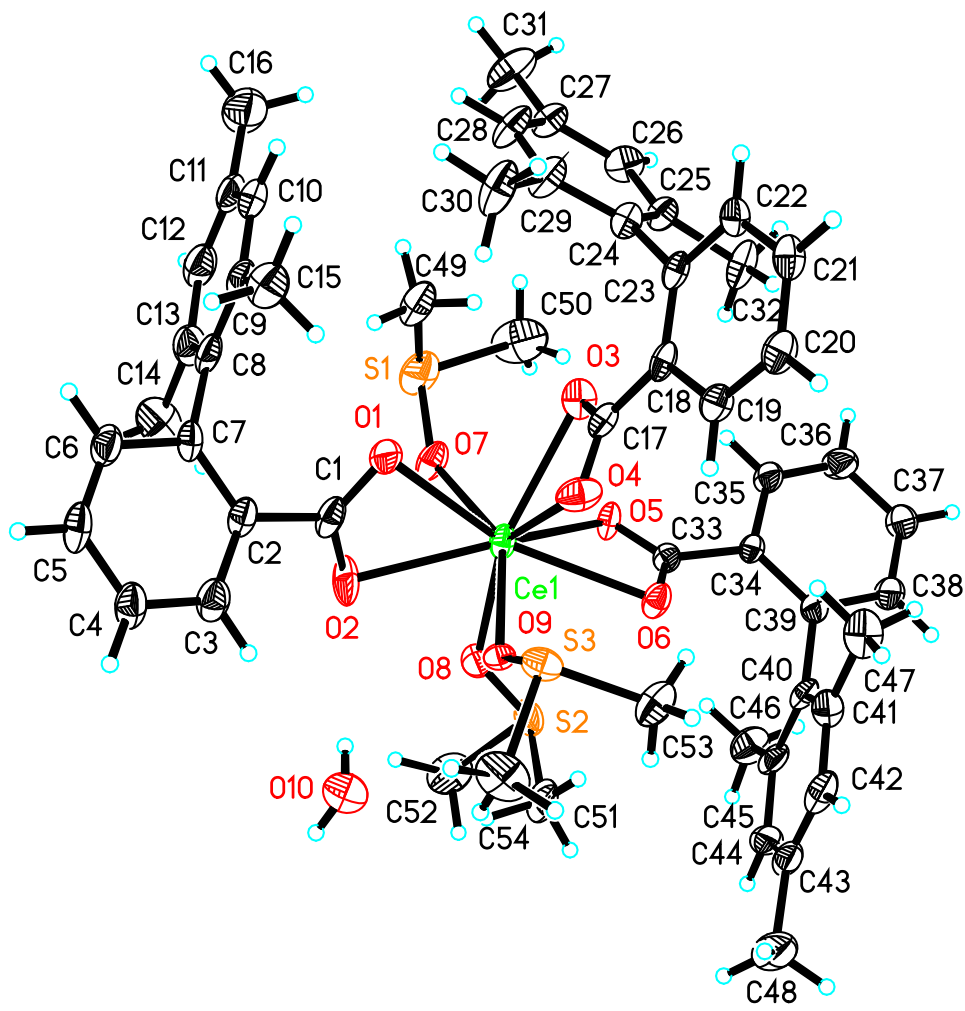

Table S6. Crystal data and structure refinement for complex $\mathbf{5 6}$.

Identification code

Empirical formula

Formula weight

Temperature

Wavelength

Crystal system

Space group

Unit cell dimensions

$106.887(2)^{\circ}$

Volume

$\mathrm{Z}$

Density (calculated) complex 56

C54 H64 Ce O9.50 S3

1101.35

193(2) K

$0.71073 \AA$

Monoclinic

$\mathrm{C} 2 / \mathrm{c}$

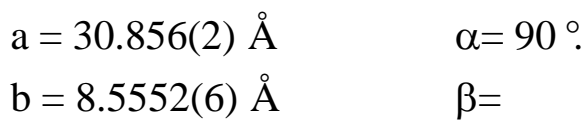

$\mathrm{c}=42.208(3) \AA \quad \gamma=90^{\circ}$.

10661.7(13) $\AA^{3}$

8

$1.372 \mathrm{Mg} / \mathrm{m}^{3}$ 
Absorption coefficient

$\mathrm{F}(000)$

Crystal size

Theta range for data collection

Index ranges

Reflections collected

Independent reflections

Completeness to theta $=25.242^{\circ}$

Absorption correction

Max. and min. transmission

Refinement method

Data / restraints / parameters

Goodness-of-fit on $\mathrm{F}^{2}$

Final $\mathrm{R}$ indices [I $>2 \operatorname{sigma}(\mathrm{I})]$

$\mathrm{R}$ indices (all data)

Extinction coefficient

Largest diff. peak and hole
$1.025 \mathrm{~mm}^{-1}$

4560

$0.170 \times 0.110 \times 0.060 \mathrm{~mm}^{3}$

1.931 to $24.999^{\circ}$.

$-36<=\mathrm{h}<=33,-10<=\mathrm{k}<=10,-50<=\mathrm{l}<=50$

46110

$9364[\mathrm{R}(\mathrm{int})=0.0853]$

$96.9 \%$

Semi-empirical from equivalents

0.7456 and 0.6223

Full-matrix least-squares on $\mathrm{F}^{2}$

9364 / 92 / 694

1.205

$\mathrm{R} 1=0.0819, \mathrm{wR} 2=0.1533$

$\mathrm{R} 1=0.1246, \mathrm{wR} 2=0.1696$

$\mathrm{n} / \mathrm{a}$

1.214 and -0.765 e. $\AA^{-3}$ 
Table S7. Atomic coordinates ( $\left.\quad 10^{4}\right)$ and equivalent isotropic displacement parameters $\left(\AA^{2} \times 10^{3}\right)$ for complex 56. U(eq) is defined as one third of the trace of the orthogonalized $\mathrm{U}^{\mathrm{ij}}$ tensor.

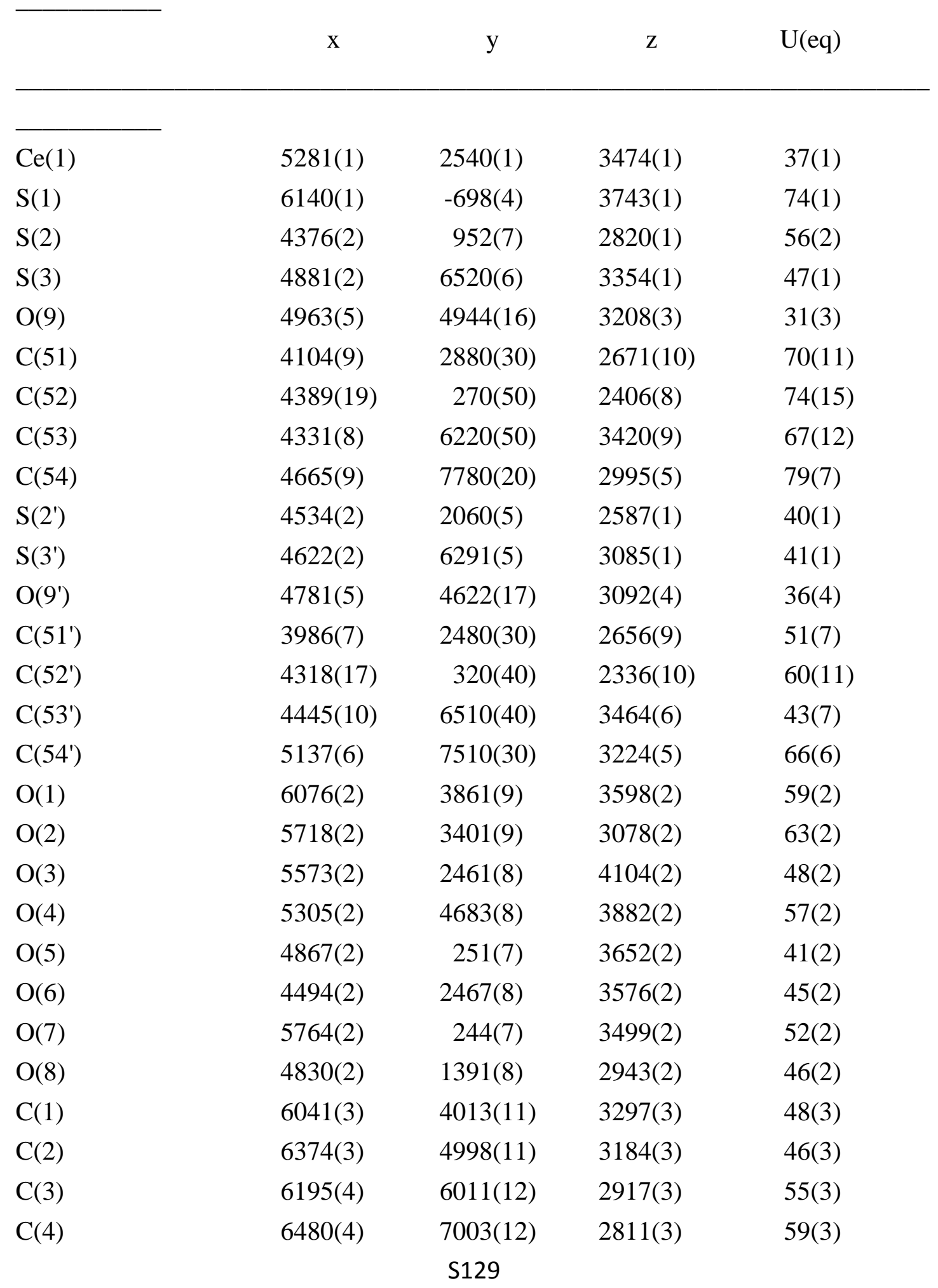




\begin{tabular}{|c|c|c|c|c|}
\hline$C(5)$ & $6935(4)$ & $6977(12)$ & 2963(3) & $60(3)$ \\
\hline$C(6)$ & $7119(3)$ & $5992(13)$ & $3221(3)$ & $55(3)$ \\
\hline$C(7)$ & $6835(3)$ & $4970(11)$ & $3334(2)$ & $45(2)$ \\
\hline$C(8)$ & $7075(3)$ & $3847(12)$ & $3598(3)$ & $49(3)$ \\
\hline $\mathrm{C}(9)$ & $7248(3)$ & $4335(12)$ & $3927(3)$ & $49(3)$ \\
\hline $\mathrm{C}(10)$ & $7492(3)$ & $3286(15)$ & $4166(3)$ & $60(3)$ \\
\hline $\mathrm{C}(11)$ & $7580(3)$ & $1769(15)$ & $4084(3)$ & $60(3)$ \\
\hline$C(12)$ & $7408(4)$ & $1310(14)$ & $3759(3)$ & $64(3)$ \\
\hline$C(13)$ & $7151(4)$ & $2274(13)$ & $3512(3)$ & $58(3)$ \\
\hline $\mathrm{C}(14)$ & $6956(5)$ & $1728(14)$ & $3160(3)$ & $83(4)$ \\
\hline$C(15)$ & 7158(4) & $5975(12)$ & $4027(3)$ & $65(3)$ \\
\hline$C(16)$ & $7838(4)$ & $636(16)$ & $4347(3)$ & $94(5)$ \\
\hline $\mathrm{C}(17)$ & $5467(3)$ & $3844(12)$ & 4134(3) & $43(2)$ \\
\hline $\mathrm{C}(18)$ & $5521(3)$ & $4557(10)$ & $4465(2)$ & $38(2)$ \\
\hline C(19) & $5251(3)$ & $5890(10)$ & $4477(2)$ & $43(2)$ \\
\hline$C(20)$ & $5271(3)$ & $6595(12)$ & $4771(3)$ & $51(3)$ \\
\hline$C(21)$ & $5560(3)$ & $6070(11)$ & $5062(3)$ & $47(3)$ \\
\hline $\mathrm{C}(22)$ & $5829(3)$ & $4733(11)$ & $5057(2)$ & $42(2)$ \\
\hline$C(23)$ & $5798(3)$ & $3990(11)$ & $4760(2)$ & $39(2)$ \\
\hline $\mathrm{C}(24)$ & $6106(3)$ & 2592(11) & $4789(2)$ & $40(2)$ \\
\hline$C(25)$ & $5957(3)$ & $1098(10)$ & $4841(2)$ & $39(2)$ \\
\hline$C(26)$ & $6250(3)$ & $-145(11)$ & $4903(2)$ & $51(3)$ \\
\hline $\mathrm{O}(10)$ & 5000 & $5656(13)$ & 2500 & $66(3)$ \\
\hline$C(27)$ & $6699(3)$ & $31(11)$ & 4904(3) & $48(3)$ \\
\hline$C(28)$ & $6837(3)$ & $1496(11)$ & $4844(3)$ & $58(3)$ \\
\hline C(29) & $6551(3)$ & $2787(10)$ & 4781(3) & $50(3)$ \\
\hline$C(30)$ & $6713(4)$ & $4365(12)$ & $4698(3)$ & $71(4)$ \\
\hline $\mathrm{C}(31)$ & 7011(4) & $-1374(12)$ & $4968(3)$ & $77(4)$ \\
\hline$C(32)$ & $5460(3)$ & $850(12)$ & $4823(3)$ & $60(3)$ \\
\hline$C(33)$ & $4548(3)$ & $1088(11)$ & $3678(2)$ & $35(2)$ \\
\hline$C(34)$ & $4232(3)$ & $373(10)$ & $3853(2)$ & $34(2)$ \\
\hline$C(35)$ & $4404(3)$ & $-738(11)$ & 4094(2) & $43(2)$ \\
\hline$C(36)$ & $4154(3)$ & $-1421(13)$ & $4269(3)$ & $59(3)$ \\
\hline$C(37)$ & $3700(3)$ & $-990(14)$ & $4207(3)$ & $62(3)$ \\
\hline$C(38)$ & $3516(3)$ & $80(14)$ & $3966(2)$ & $58(3)$ \\
\hline C(39) & $3782(3)$ & $818(12)$ & $3791(2)$ & $42(2)$ \\
\hline $\mathrm{C}(40)$ & $3543(3)$ & $1967(10)$ & $3523(2)$ & $38(2)$ \\
\hline \multirow[t]{2}{*}{$C(41)$} & $3528(4)$ & $3579(12)$ & $3599(3)$ & $51(3)$ \\
\hline & \multicolumn{4}{|c|}{ S130 } \\
\hline
\end{tabular}




$\begin{array}{llrrr}\mathrm{C}(42) & 3290(3) & 4566(13) & 3354(3) & 60(3) \\ \mathrm{C}(43) & 3092(3) & 4089(14) & 3034(3) & 56(3) \\ \mathrm{C}(44) & 3107(3) & 2523(16) & 2963(2) & 57(3) \\ \mathrm{C}(45) & 3338(3) & 1461(12) & 3206(3) & 46(3) \\ \mathrm{C}(46) & 3343(4) & -291(12) & 3110(3) & 64(3) \\ \mathrm{C}(47) & 3752(4) & 4154(14) & 3945(3) & 72(4) \\ \mathrm{C}(48) & 2858(4) & 5202(16) & 2761(3) & 94(5) \\ \mathrm{C}(49) & 6465(3) & 644(14) & 4036(3) & 74(4) \\ \mathrm{C}(50) & 5869(5) & -1605(16) & 4029(4) & 116(6)\end{array}$


Table S8. Bond lengths $[\AA]$ and angles $\left[{ }^{\circ}\right]$ for complex 56.

\begin{tabular}{|c|c|}
\hline $\mathrm{Ce}(1)-\mathrm{O}(9)$ & $2.410(13)$ \\
\hline $\mathrm{Ce}(1)-\mathrm{O}(7)$ & $2.450(6)$ \\
\hline $\mathrm{Ce}(1)-\mathrm{O}(8)$ & $2.472(6)$ \\
\hline $\mathrm{Ce}(1)-\mathrm{O}(4)$ & $2.501(7)$ \\
\hline $\mathrm{Ce}(1)-\mathrm{O}(2)$ & $2.543(6)$ \\
\hline $\mathrm{Ce}(1)-\mathrm{O}(3)$ & $2.551(6)$ \\
\hline $\mathrm{Ce}(1)-\mathrm{O}(5)$ & $2.567(6)$ \\
\hline $\mathrm{Ce}(1)-\mathrm{O}(6)$ & $2.586(5)$ \\
\hline $\mathrm{Ce}(1)-\mathrm{O}\left(9^{\prime}\right)$ & $2.588(14)$ \\
\hline $\mathrm{Ce}(1)-\mathrm{O}(1)$ & $2.613(6)$ \\
\hline $\mathrm{Ce}(1)-\mathrm{C}(17)$ & $2.901(10)$ \\
\hline $\mathrm{Ce}(1)-\mathrm{C}(33)$ & $2.920(8)$ \\
\hline $\mathrm{S}(1)-\mathrm{O}(7)$ & $1.536(7)$ \\
\hline$S(1)-C(49)$ & $1.770(12)$ \\
\hline$S(1)-C(50)$ & $1.828(13)$ \\
\hline $\mathrm{S}(2)-\mathrm{O}(8)$ & $1.396(8)$ \\
\hline$S(2)-C(52)$ & $1.852(19)$ \\
\hline$S(2)-C(51)$ & $1.875(18)$ \\
\hline $\mathrm{S}(3)-\mathrm{O}(9)$ & $1.533(15)$ \\
\hline$S(3)-C(53)$ & $1.819(17)$ \\
\hline$S(3)-C(54)$ & $1.820(15)$ \\
\hline $\mathrm{C}(51)-\mathrm{H}(51 \mathrm{~A})$ & 0.9800 \\
\hline $\mathrm{C}(51)-\mathrm{H}(51 \mathrm{~B})$ & 0.9800 \\
\hline $\mathrm{C}(51)-\mathrm{H}(51 \mathrm{C})$ & 0.9800 \\
\hline $\mathrm{C}(52)-\mathrm{H}(52 \mathrm{~A})$ & 0.9800 \\
\hline $\mathrm{C}(52)-\mathrm{H}(52 \mathrm{~B})$ & 0.9800 \\
\hline $\mathrm{C}(52)-\mathrm{H}(52 \mathrm{C})$ & 0.9800 \\
\hline $\mathrm{C}(53)-\mathrm{H}(53 \mathrm{~A})$ & 0.9800 \\
\hline $\mathrm{C}(53)-\mathrm{H}(53 \mathrm{~B})$ & 0.9800 \\
\hline $\mathrm{C}(53)-\mathrm{H}(53 \mathrm{C})$ & 0.9800 \\
\hline $\mathrm{C}(54)-\mathrm{H}(54 \mathrm{~A})$ & 0.9800 \\
\hline $\mathrm{C}(54)-\mathrm{H}(54 \mathrm{~B})$ & 0.9800 \\
\hline $\mathrm{C}(54)-\mathrm{H}(54 \mathrm{C})$ & 0.9800 \\
\hline $\mathrm{S}\left(2^{\prime}\right)-\mathrm{O}(8)$ & $1.618(8)$ \\
\hline $\mathrm{S}\left(2^{\prime}\right)-\mathrm{C}\left(51^{\prime}\right)$ & $1.831(17)$ \\
\hline$S\left(2^{\prime}\right)-C\left(52^{\prime}\right)$ & $1.84(2)$ \\
\hline
\end{tabular}




\begin{tabular}{|c|c|}
\hline $\mathrm{S}\left(3^{\prime}\right)-\mathrm{O}\left(9^{\prime}\right)$ & $1.508(15)$ \\
\hline$S\left(3^{\prime}\right)-C\left(53^{\prime}\right)$ & $1.843(18)$ \\
\hline$S\left(3^{\prime}\right)-C\left(54^{\prime}\right)$ & $1.849(15)$ \\
\hline $\mathrm{C}\left(51^{\prime}\right)-\mathrm{H}(51 \mathrm{D})$ & 0.9800 \\
\hline $\mathrm{C}\left(51^{\prime}\right)-\mathrm{H}(51 \mathrm{E})$ & 0.9800 \\
\hline $\mathrm{C}\left(51^{\prime}\right)-\mathrm{H}(51 \mathrm{~F})$ & 0.9800 \\
\hline $\mathrm{C}\left(52^{\prime}\right)-\mathrm{H}(52 \mathrm{D})$ & 0.9800 \\
\hline $\mathrm{C}\left(52^{\prime}\right)-\mathrm{H}(52 \mathrm{E})$ & 0.9800 \\
\hline $\mathrm{C}\left(52^{\prime}\right)-\mathrm{H}(52 \mathrm{~F})$ & 0.9800 \\
\hline $\mathrm{C}\left(53^{\prime}\right)-\mathrm{H}(53 \mathrm{D})$ & 0.9800 \\
\hline $\mathrm{C}\left(53^{\prime}\right)-\mathrm{H}(53 \mathrm{E})$ & 0.9800 \\
\hline $\mathrm{C}\left(53^{\prime}\right)-\mathrm{H}(53 \mathrm{~F})$ & 0.9800 \\
\hline $\mathrm{C}\left(54^{\prime}\right)-\mathrm{H}(54 \mathrm{D})$ & 0.9800 \\
\hline $\mathrm{C}\left(54^{\prime}\right)-\mathrm{H}(54 \mathrm{E})$ & 0.9800 \\
\hline $\mathrm{C}\left(54^{\prime}\right)-\mathrm{H}(54 \mathrm{~F})$ & 0.9800 \\
\hline $\mathrm{O}(1)-\mathrm{C}(1)$ & $1.249(12)$ \\
\hline $\mathrm{O}(2)-\mathrm{C}(1)$ & $1.261(12)$ \\
\hline $\mathrm{O}(3)-\mathrm{C}(17)$ & $1.244(11)$ \\
\hline $\mathrm{O}(4)-\mathrm{C}(17)$ & $1.261(11)$ \\
\hline $\mathrm{O}(5)-\mathrm{C}(33)$ & $1.246(10)$ \\
\hline $\mathrm{O}(6)-\mathrm{C}(33)$ & $1.251(10)$ \\
\hline $\mathrm{C}(1)-\mathrm{C}(2)$ & $1.512(13)$ \\
\hline$C(2)-C(7)$ & $1.378(13)$ \\
\hline$C(2)-C(3)$ & $1.401(13)$ \\
\hline$C(3)-C(4)$ & $1.386(13)$ \\
\hline $\mathrm{C}(3)-\mathrm{H}(3)$ & 0.9500 \\
\hline$C(4)-C(5)$ & $1.366(14)$ \\
\hline $\mathrm{C}(4)-\mathrm{H}(4)$ & 0.9500 \\
\hline$C(5)-C(6)$ & $1.364(14)$ \\
\hline $\mathrm{C}(5)-\mathrm{H}(5)$ & 0.9500 \\
\hline$C(6)-C(7)$ & $1.414(13)$ \\
\hline $\mathrm{C}(6)-\mathrm{H}(6)$ & 0.9500 \\
\hline$C(7)-C(8)$ & $1.495(13)$ \\
\hline $\mathrm{C}(8)-\mathrm{C}(9)$ & $1.398(14)$ \\
\hline$C(8)-C(13)$ & $1.430(14)$ \\
\hline$C(9)-C(10)$ & $1.396(14)$ \\
\hline $\mathrm{C}(9)-\mathrm{C}(15)$ & $1.514(14)$ \\
\hline$C(10)-C(11)$ & $1.391(15)$ \\
\hline
\end{tabular}




\begin{tabular}{|c|c|}
\hline $\mathrm{C}(10)-\mathrm{H}(10)$ & 0.9500 \\
\hline $\mathrm{C}(11)-\mathrm{C}(12)$ & $1.374(16)$ \\
\hline$C(11)-C(16)$ & $1.515(15)$ \\
\hline$C(12)-C(13)$ & $1.385(14)$ \\
\hline $\mathrm{C}(12)-\mathrm{H}(12)$ & 0.9500 \\
\hline$C(13)-C(14)$ & $1.505(15)$ \\
\hline $\mathrm{C}(14)-\mathrm{H}(14 \mathrm{~A})$ & 0.9800 \\
\hline $\mathrm{C}(14)-\mathrm{H}(14 \mathrm{~B})$ & 0.9800 \\
\hline $\mathrm{C}(14)-\mathrm{H}(14 \mathrm{C})$ & 0.9800 \\
\hline $\mathrm{C}(15)-\mathrm{H}(15 \mathrm{~A})$ & 0.9800 \\
\hline $\mathrm{C}(15)-\mathrm{H}(15 \mathrm{~B})$ & 0.9800 \\
\hline $\mathrm{C}(15)-\mathrm{H}(15 \mathrm{C})$ & 0.9800 \\
\hline $\mathrm{C}(16)-\mathrm{H}(16 \mathrm{~A})$ & 0.9800 \\
\hline $\mathrm{C}(16)-\mathrm{H}(16 \mathrm{~B})$ & 0.9800 \\
\hline $\mathrm{C}(16)-\mathrm{H}(16 \mathrm{C})$ & 0.9800 \\
\hline $\mathrm{C}(17)-\mathrm{C}(18)$ & $1.486(13)$ \\
\hline $\mathrm{C}(18)-\mathrm{C}(23)$ & $1.379(13)$ \\
\hline C(18)-C(19) & $1.421(12)$ \\
\hline $\mathrm{C}(19)-\mathrm{C}(20)$ & $1.364(13)$ \\
\hline $\mathrm{C}(19)-\mathrm{H}(19)$ & 0.9500 \\
\hline $\mathrm{C}(20)-\mathrm{C}(21)$ & $1.368(13)$ \\
\hline $\mathrm{C}(20)-\mathrm{H}(20)$ & 0.9500 \\
\hline $\mathrm{C}(21)-\mathrm{C}(22)$ & $1.418(12)$ \\
\hline $\mathrm{C}(21)-\mathrm{H}(21)$ & 0.9500 \\
\hline $\mathrm{C}(22)-\mathrm{C}(23)$ & $1.383(12)$ \\
\hline $\mathrm{C}(22)-\mathrm{H}(22)$ & 0.9500 \\
\hline $\mathrm{C}(23)-\mathrm{C}(24)$ & $1.510(13)$ \\
\hline $\mathrm{C}(24)-\mathrm{C}(29)$ & $1.394(12)$ \\
\hline $\mathrm{C}(24)-\mathrm{C}(25)$ & $1.398(13)$ \\
\hline$C(25)-C(26)$ & $1.371(12)$ \\
\hline $\mathrm{C}(25)-\mathrm{C}(32)$ & $1.527(12)$ \\
\hline $\mathrm{C}(26)-\mathrm{C}(27)$ & $1.391(13)$ \\
\hline $\mathrm{C}(26)-\mathrm{H}(26)$ & 0.9500 \\
\hline $\mathrm{O}(10)-\mathrm{H}(10 \mathrm{~A})$ & 0.8697 \\
\hline $\mathrm{O}(10)-\mathrm{H}(10 \mathrm{~B})$ & 0.8699 \\
\hline $\mathrm{C}(27)-\mathrm{C}(28)$ & $1.370(13)$ \\
\hline $\mathrm{C}(27)-\mathrm{C}(31)$ & $1.515(13)$ \\
\hline $\mathrm{C}(28)-\mathrm{C}(29)$ & $1.389(12)$ \\
\hline
\end{tabular}




\begin{tabular}{|c|c|}
\hline $\mathrm{C}(28)-\mathrm{H}(28)$ & 0.9500 \\
\hline $\mathrm{C}(29)-\mathrm{C}(30)$ & $1.516(13)$ \\
\hline $\mathrm{C}(30)-\mathrm{H}(30 \mathrm{~A})$ & 0.9800 \\
\hline $\mathrm{C}(30)-\mathrm{H}(30 \mathrm{~B})$ & 0.9800 \\
\hline $\mathrm{C}(30)-\mathrm{H}(30 \mathrm{C})$ & 0.9800 \\
\hline $\mathrm{C}(31)-\mathrm{H}(31 \mathrm{~A})$ & 0.9800 \\
\hline $\mathrm{C}(31)-\mathrm{H}(31 \mathrm{~B})$ & 0.9800 \\
\hline $\mathrm{C}(31)-\mathrm{H}(31 \mathrm{C})$ & 0.9800 \\
\hline $\mathrm{C}(32)-\mathrm{H}(32 \mathrm{~A})$ & 0.9800 \\
\hline $\mathrm{C}(32)-\mathrm{H}(32 \mathrm{~B})$ & 0.9800 \\
\hline $\mathrm{C}(32)-\mathrm{H}(32 \mathrm{C})$ & 0.9800 \\
\hline $\mathrm{C}(33)-\mathrm{C}(34)$ & $1.515(11)$ \\
\hline $\mathrm{C}(34)-\mathrm{C}(35)$ & $1.380(12)$ \\
\hline $\mathrm{C}(34)-\mathrm{C}(39)$ & $1.391(12)$ \\
\hline $\mathrm{C}(35)-\mathrm{C}(36)$ & $1.346(13)$ \\
\hline $\mathrm{C}(35)-\mathrm{H}(35)$ & 0.9500 \\
\hline $\mathrm{C}(36)-\mathrm{C}(37)$ & $1.398(14)$ \\
\hline $\mathrm{C}(36)-\mathrm{H}(36)$ & 0.9500 \\
\hline $\mathrm{C}(37)-\mathrm{C}(38)$ & $1.362(14)$ \\
\hline $\mathrm{C}(37)-\mathrm{H}(37)$ & 0.9500 \\
\hline $\mathrm{C}(38)-\mathrm{C}(39)$ & $1.405(12)$ \\
\hline $\mathrm{C}(38)-\mathrm{H}(38)$ & 0.9500 \\
\hline $\mathrm{C}(39)-\mathrm{C}(40)$ & $1.518(12)$ \\
\hline $\mathrm{C}(40)-\mathrm{C}(45)$ & $1.374(13)$ \\
\hline $\mathrm{C}(40)-\mathrm{C}(41)$ & $1.419(13)$ \\
\hline $\mathrm{C}(41)-\mathrm{C}(42)$ & $1.370(14)$ \\
\hline $\mathrm{C}(41)-\mathrm{C}(47)$ & $1.506(14)$ \\
\hline $\mathrm{C}(42)-\mathrm{C}(43)$ & $1.371(15)$ \\
\hline $\mathrm{C}(42)-\mathrm{H}(42)$ & 0.9500 \\
\hline $\mathrm{C}(43)-\mathrm{C}(44)$ & $1.377(16)$ \\
\hline $\mathrm{C}(43)-\mathrm{C}(48)$ & $1.508(14)$ \\
\hline $\mathrm{C}(44)-\mathrm{C}(45)$ & $1.399(14)$ \\
\hline $\mathrm{C}(44)-\mathrm{H}(44)$ & 0.9500 \\
\hline $\mathrm{C}(45)-\mathrm{C}(46)$ & $1.554(14)$ \\
\hline $\mathrm{C}(46)-\mathrm{H}(46 \mathrm{~A})$ & 0.9800 \\
\hline $\mathrm{C}(46)-\mathrm{H}(46 \mathrm{~B})$ & 0.9800 \\
\hline $\mathrm{C}(46)-\mathrm{H}(46 \mathrm{C})$ & 0.9800 \\
\hline $\mathrm{C}(47)-\mathrm{H}(47 \mathrm{~A})$ & 0.9800 \\
\hline
\end{tabular}




\begin{tabular}{|c|c|}
\hline $\mathrm{C}(47)-\mathrm{H}(47 \mathrm{~B})$ & 0.9800 \\
\hline $\mathrm{C}(47)-\mathrm{H}(47 \mathrm{C})$ & 0.9800 \\
\hline $\mathrm{C}(48)-\mathrm{H}(48 \mathrm{~A})$ & 0.9800 \\
\hline $\mathrm{C}(48)-\mathrm{H}(48 \mathrm{~B})$ & 0.9800 \\
\hline $\mathrm{C}(48)-\mathrm{H}(48 \mathrm{C})$ & 0.9800 \\
\hline C(49)-H(49A) & 0.9800 \\
\hline $\mathrm{C}(49)-\mathrm{H}(49 \mathrm{~B})$ & 0.9800 \\
\hline $\mathrm{C}(49)-\mathrm{H}(49 \mathrm{C})$ & 0.9800 \\
\hline $\mathrm{C}(50)-\mathrm{H}(50 \mathrm{~A})$ & 0.9800 \\
\hline $\mathrm{C}(50)-\mathrm{H}(50 \mathrm{~B})$ & 0.9800 \\
\hline $\mathrm{C}(50)-\mathrm{H}(50 \mathrm{C})$ & 0.9800 \\
\hline $\mathrm{O}(9)-\mathrm{Ce}(1)-\mathrm{O}(7)$ & $149.8(4)$ \\
\hline $\mathrm{O}(9)-\mathrm{Ce}(1)-\mathrm{O}(8)$ & $82.6(4)$ \\
\hline $\mathrm{O}(7)-\mathrm{Ce}(1)-\mathrm{O}(8)$ & $84.0(2)$ \\
\hline $\mathrm{O}(9)-\mathrm{Ce}(1)-\mathrm{O}(4)$ & $67.8(4)$ \\
\hline $\mathrm{O}(7)-\mathrm{Ce}(1)-\mathrm{O}(4)$ & $131.4(2)$ \\
\hline $\mathrm{O}(8)-\mathrm{Ce}(1)-\mathrm{O}(4)$ & $144.3(2)$ \\
\hline $\mathrm{O}(9)-\mathrm{Ce}(1)-\mathrm{O}(2)$ & $70.6(4)$ \\
\hline $\mathrm{O}(7)-\mathrm{Ce}(1)-\mathrm{O}(2)$ & $80.1(2)$ \\
\hline $\mathrm{O}(8)-\mathrm{Ce}(1)-\mathrm{O}(2)$ & $77.6(2)$ \\
\hline $\mathrm{O}(4)-\mathrm{Ce}(1)-\mathrm{O}(2)$ & $109.0(2)$ \\
\hline $\mathrm{O}(9)-\mathrm{Ce}(1)-\mathrm{O}(3)$ & $118.7(4)$ \\
\hline $\mathrm{O}(7)-\mathrm{Ce}(1)-\mathrm{O}(3)$ & $84.7(2)$ \\
\hline $\mathrm{O}(8)-\mathrm{Ce}(1)-\mathrm{O}(3)$ & $150.5(2)$ \\
\hline $\mathrm{O}(4)-\mathrm{Ce}(1)-\mathrm{O}(3)$ & $51.0(2)$ \\
\hline $\mathrm{O}(2)-\mathrm{Ce}(1)-\mathrm{O}(3)$ & $126.9(2)$ \\
\hline $\mathrm{O}(9)-\mathrm{Ce}(1)-\mathrm{O}(5)$ & $128.1(4)$ \\
\hline $\mathrm{O}(7)-\mathrm{Ce}(1)-\mathrm{O}(5)$ & $74.0(2)$ \\
\hline $\mathrm{O}(8)-\mathrm{Ce}(1)-\mathrm{O}(5)$ & $76.4(2)$ \\
\hline $\mathrm{O}(4)-\mathrm{Ce}(1)-\mathrm{O}(5)$ & $106.0(2)$ \\
\hline $\mathrm{O}(2)-\mathrm{Ce}(1)-\mathrm{O}(5)$ & $144.8(2)$ \\
\hline $\mathrm{O}(3)-\mathrm{Ce}(1)-\mathrm{O}(5)$ & $74.3(2)$ \\
\hline $\mathrm{O}(9)-\mathrm{Ce}(1)-\mathrm{O}(6)$ & $79.8(4)$ \\
\hline $\mathrm{O}(7)-\mathrm{Ce}(1)-\mathrm{O}(6)$ & $124.2(2)$ \\
\hline $\mathrm{O}(8)-\mathrm{Ce}(1)-\mathrm{O}(6)$ & $80.4(2)$ \\
\hline $\mathrm{O}(4)-\mathrm{Ce}(1)-\mathrm{O}(6)$ & $75.2(2)$ \\
\hline $\mathrm{O}(2)-\mathrm{Ce}(1)-\mathrm{O}(6)$ & $145.0(2)$ \\
\hline
\end{tabular}




\begin{tabular}{|c|c|}
\hline $\mathrm{O}(3)-\mathrm{Ce}(1)-\mathrm{O}(6)$ & $83.68(19)$ \\
\hline $\mathrm{O}(5)-\mathrm{Ce}(1)-\mathrm{O}(6)$ & $50.35(19)$ \\
\hline $\mathrm{O}(7)-\mathrm{Ce}(1)-\mathrm{O}\left(9^{\prime}\right)$ & $145.5(4)$ \\
\hline $\mathrm{O}(8)-\mathrm{Ce}(1)-\mathrm{O}\left(9^{\prime}\right)$ & $68.5(3)$ \\
\hline $\mathrm{O}(4)-\mathrm{Ce}(1)-\mathrm{O}\left(9^{\prime}\right)$ & $79.5(4)$ \\
\hline $\mathrm{O}(2)-\mathrm{Ce}(1)-\mathrm{O}\left(9^{\prime}\right)$ & $74.1(4)$ \\
\hline $\mathrm{O}(3)-\mathrm{Ce}(1)-\mathrm{O}\left(9^{\prime}\right)$ & $129.3(4)$ \\
\hline $\mathrm{O}(5)-\mathrm{Ce}(1)-\mathrm{O}\left(9^{\prime}\right)$ & $116.7(4)$ \\
\hline $\mathrm{O}(6)-\mathrm{Ce}(1)-\mathrm{O}\left(9^{\prime}\right)$ & $72.6(4)$ \\
\hline $\mathrm{O}(9)-\mathrm{Ce}(1)-\mathrm{O}(1)$ & $87.5(4)$ \\
\hline $\mathrm{O}(7)-\mathrm{Ce}(1)-\mathrm{O}(1)$ & $79.2(2)$ \\
\hline $\mathrm{O}(8)-\mathrm{Ce}(1)-\mathrm{O}(1)$ & $127.1(2)$ \\
\hline $\mathrm{O}(4)-\mathrm{Ce}(1)-\mathrm{O}(1)$ & $72.9(2)$ \\
\hline $\mathrm{O}(2)-\mathrm{Ce}(1)-\mathrm{O}(1)$ & $50.2(2)$ \\
\hline $\mathrm{O}(3)-\mathrm{Ce}(1)-\mathrm{O}(1)$ & $77.0(2)$ \\
\hline $\mathrm{O}(5)-\mathrm{Ce}(1)-\mathrm{O}(1)$ & $142.1(2)$ \\
\hline $\mathrm{O}(6)-\mathrm{Ce}(1)-\mathrm{O}(1)$ & $148.1(2)$ \\
\hline $\mathrm{O}\left(9^{\prime}\right)-\mathrm{Ce}(1)-\mathrm{O}(1)$ & $100.5(4)$ \\
\hline $\mathrm{O}(9)-\mathrm{Ce}(1)-\mathrm{C}(17)$ & $93.4(4)$ \\
\hline $\mathrm{O}(7)-\mathrm{Ce}(1)-\mathrm{C}(17)$ & $108.6(3)$ \\
\hline $\mathrm{O}(8)-\mathrm{Ce}(1)-\mathrm{C}(17)$ & $157.8(2)$ \\
\hline $\mathrm{O}(4)-\mathrm{Ce}(1)-\mathrm{C}(17)$ & $25.7(2)$ \\
\hline $\mathrm{O}(2)-\mathrm{Ce}(1)-\mathrm{C}(17)$ & $121.7(2)$ \\
\hline $\mathrm{O}(3)-\mathrm{Ce}(1)-\mathrm{C}(17)$ & $25.3(2)$ \\
\hline $\mathrm{O}(5)-\mathrm{Ce}(1)-\mathrm{C}(17)$ & $89.4(2)$ \\
\hline $\mathrm{O}(6)-\mathrm{Ce}(1)-\mathrm{C}(17)$ & $77.5(2)$ \\
\hline $\mathrm{O}\left(9^{\prime}\right)-\mathrm{Ce}(1)-\mathrm{C}(17)$ & $104.4(4)$ \\
\hline $\mathrm{O}(1)-\mathrm{Ce}(1)-\mathrm{C}(17)$ & $74.2(2)$ \\
\hline $\mathrm{O}(9)-\mathrm{Ce}(1)-\mathrm{C}(33)$ & $104.8(4)$ \\
\hline $\mathrm{O}(7)-\mathrm{Ce}(1)-\mathrm{C}(33)$ & $99.2(2)$ \\
\hline $\mathrm{O}(8)-\mathrm{Ce}(1)-\mathrm{C}(33)$ & $79.5(2)$ \\
\hline $\mathrm{O}(4)-\mathrm{Ce}(1)-\mathrm{C}(33)$ & $88.8(2)$ \\
\hline $\mathrm{O}(2)-\mathrm{Ce}(1)-\mathrm{C}(33)$ & $157.1(2)$ \\
\hline $\mathrm{O}(3)-\mathrm{Ce}(1)-\mathrm{C}(33)$ & $75.5(2)$ \\
\hline $\mathrm{O}(5)-\mathrm{Ce}(1)-\mathrm{C}(33)$ & $25.2(2)$ \\
\hline $\mathrm{O}(6)-\mathrm{Ce}(1)-\mathrm{C}(33)$ & $25.3(2)$ \\
\hline $\mathrm{O}\left(9^{\prime}\right)-\mathrm{Ce}(1)-\mathrm{C}(33)$ & $96.0(4)$ \\
\hline $\mathrm{O}(1)-\mathrm{Ce}(1)-\mathrm{C}(33)$ & $152.5(2)$ \\
\hline
\end{tabular}




\begin{tabular}{|c|c|}
\hline (1)-C(33) & $80.5(2)$ \\
\hline $\mathrm{O}(7)-\mathrm{S}(1)-\mathrm{C}(49)$ & $107.0(5)$ \\
\hline $\mathrm{O}(7)-\mathrm{S}(1)-\mathrm{C}(50)$ & $105.3(5)$ \\
\hline$C(49)-S(1)-C(50)$ & $95.1(6)$ \\
\hline $\mathrm{O}(8)-\mathrm{S}(2)-\mathrm{C}(52)$ & $98.2(16)$ \\
\hline $\mathrm{O}(8)-\mathrm{S}(2)-\mathrm{C}(51)$ & $101.0(10)$ \\
\hline$C(52)-S(2)-C(51)$ & $95(2)$ \\
\hline $\mathrm{O}(9)-\mathrm{S}(3)-\mathrm{C}(53)$ & $101.9(14)$ \\
\hline $\mathrm{O}(9)-\mathrm{S}(3)-\mathrm{C}(54)$ & $104.4(9)$ \\
\hline$C(53)-S(3)-C(54)$ & $94.5(16)$ \\
\hline $\mathrm{S}(3)-\mathrm{O}(9)-\mathrm{Ce}(1)$ & $131.0(8)$ \\
\hline $\mathrm{S}(2)-\mathrm{C}(51)-\mathrm{H}(51 \mathrm{~A})$ & 109.5 \\
\hline $\mathrm{S}(2)-\mathrm{C}(51)-\mathrm{H}(51 \mathrm{~B})$ & 109.5 \\
\hline $\mathrm{H}(51 \mathrm{~A})-\mathrm{C}(51)-\mathrm{H}(51 \mathrm{~B})$ & 109.5 \\
\hline$S(2)-C(51)-H(51 C)$ & 109.5 \\
\hline $\mathrm{H}(51 \mathrm{~A})-\mathrm{C}(51)-\mathrm{H}(51 \mathrm{C})$ & 109.5 \\
\hline $\mathrm{H}(51 \mathrm{~B})-\mathrm{C}(51)-\mathrm{H}(51 \mathrm{C})$ & 109.5 \\
\hline $\mathrm{S}(2)-\mathrm{C}(52)-\mathrm{H}(52 \mathrm{~A})$ & 109.5 \\
\hline $\mathrm{S}(2)-\mathrm{C}(52)-\mathrm{H}(52 \mathrm{~B})$ & 109.5 \\
\hline $\mathrm{H}(52 \mathrm{~A})-\mathrm{C}(52)-\mathrm{H}(52 \mathrm{~B})$ & 109.5 \\
\hline $\mathrm{S}(2)-\mathrm{C}(52)-\mathrm{H}(52 \mathrm{C})$ & 109.5 \\
\hline $\mathrm{H}(52 \mathrm{~A})-\mathrm{C}(52)-\mathrm{H}(52 \mathrm{C})$ & 109.5 \\
\hline $\mathrm{H}(52 \mathrm{~B})-\mathrm{C}(52)-\mathrm{H}(52 \mathrm{C})$ & 109.5 \\
\hline $\mathrm{S}(3)-\mathrm{C}(53)-\mathrm{H}(53 \mathrm{~A})$ & 109.5 \\
\hline $\mathrm{S}(3)-\mathrm{C}(53)-\mathrm{H}(53 \mathrm{~B})$ & 109.5 \\
\hline $\mathrm{H}(53 \mathrm{~A})-\mathrm{C}(53)-\mathrm{H}(53 \mathrm{~B})$ & 109.5 \\
\hline $\mathrm{S}(3)-\mathrm{C}(53)-\mathrm{H}(53 \mathrm{C})$ & 109.5 \\
\hline $\mathrm{H}(53 \mathrm{~A})-\mathrm{C}(53)-\mathrm{H}(53 \mathrm{C})$ & 109.5 \\
\hline $\mathrm{H}(53 \mathrm{~B})-\mathrm{C}(53)-\mathrm{H}(53 \mathrm{C})$ & 109.5 \\
\hline $\mathrm{S}(3)-\mathrm{C}(54)-\mathrm{H}(54 \mathrm{~A})$ & 109.5 \\
\hline $\mathrm{S}(3)-\mathrm{C}(54)-\mathrm{H}(54 \mathrm{~B})$ & 109.5 \\
\hline $\mathrm{H}(54 \mathrm{~A})-\mathrm{C}(54)-\mathrm{H}(54 \mathrm{~B})$ & 109.5 \\
\hline $\mathrm{S}(3)-\mathrm{C}(54)-\mathrm{H}(54 \mathrm{C})$ & 109.5 \\
\hline $\mathrm{H}(54 \mathrm{~A})-\mathrm{C}(54)-\mathrm{H}(54 \mathrm{C})$ & 109.5 \\
\hline $\mathrm{H}(54 \mathrm{~B})-\mathrm{C}(54)-\mathrm{H}(54 \mathrm{C})$ & 109.5 \\
\hline $\mathrm{O}(8)-\mathrm{S}\left(2^{\prime}\right)-\mathrm{C}\left(51^{\prime}\right)$ & $102.8(12)$ \\
\hline $\mathrm{O}(8)-\mathrm{S}\left(2^{\prime}\right)-\mathrm{C}\left(52^{\prime}\right)$ & $105.1(17)$ \\
\hline$C\left(51^{\prime}\right)-S\left(2^{\prime}\right)-C\left(52^{\prime}\right)$ & $93.1(19)$ \\
\hline
\end{tabular}




\begin{tabular}{|c|c|}
\hline $\mathrm{O}\left(9^{\prime}\right)-\mathrm{S}\left(3^{\prime}\right)-\mathrm{C}\left(53^{\prime}\right)$ & 10 \\
\hline $\mathrm{O}\left(9^{\prime}\right)-\mathrm{S}\left(3^{\prime}\right)-\mathrm{C}\left(54^{\prime}\right)$ & $106.4(10)$ \\
\hline $\mathrm{C}\left(53^{\prime}\right)-\mathrm{S}\left(3^{\prime}\right)-\mathrm{C}\left(54^{\prime}\right)$ & $96.4(11)$ \\
\hline $\mathrm{S}\left(3^{\prime}\right)-\mathrm{O}\left(9^{\prime}\right)-\mathrm{Ce}(1)$ & $142.8(8)$ \\
\hline $\mathrm{S}\left(2^{\prime}\right)-\mathrm{C}\left(51^{\prime}\right)-\mathrm{H}(51 \mathrm{D})$ & 109.5 \\
\hline $\mathrm{S}\left(2^{\prime}\right)-\mathrm{C}\left(51^{\prime}\right)-\mathrm{H}(51 \mathrm{E})$ & 109.5 \\
\hline $\mathrm{H}(51 \mathrm{D})-\mathrm{C}\left(51^{\prime}\right)-\mathrm{H}(51 \mathrm{E})$ & 109.5 \\
\hline $\mathrm{S}\left(2^{\prime}\right)-\mathrm{C}\left(51^{\prime}\right)-\mathrm{H}(51 \mathrm{~F})$ & 109.5 \\
\hline $\mathrm{H}(51 \mathrm{D})-\mathrm{C}\left(51^{\prime}\right)-\mathrm{H}(51 \mathrm{~F})$ & 109.5 \\
\hline $\mathrm{H}(51 \mathrm{E})-\mathrm{C}\left(51^{\prime}\right)-\mathrm{H}(51 \mathrm{~F})$ & 109.5 \\
\hline $\mathrm{S}\left(2^{\prime}\right)-\mathrm{C}\left(52^{\prime}\right)-\mathrm{H}(52 \mathrm{D})$ & 109.5 \\
\hline $\mathrm{S}\left(2^{\prime}\right)-\mathrm{C}\left(52^{\prime}\right)-\mathrm{H}(52 \mathrm{E})$ & 109.5 \\
\hline $\mathrm{H}(52 \mathrm{D})-\mathrm{C}\left(52^{\prime}\right)-\mathrm{H}(52 \mathrm{E})$ & 109.5 \\
\hline $\mathrm{S}\left(2^{\prime}\right)-\mathrm{C}\left(52^{\prime}\right)-\mathrm{H}(52 \mathrm{~F})$ & 109.5 \\
\hline $\mathrm{H}(52 \mathrm{D})-\mathrm{C}\left(52^{\prime}\right)-\mathrm{H}(52 \mathrm{~F})$ & 109.5 \\
\hline $\mathrm{H}(52 \mathrm{E})-\mathrm{C}\left(52^{\prime}\right)-\mathrm{H}(52 \mathrm{~F})$ & 109.5 \\
\hline $\mathrm{S}\left(3^{\prime}\right)-\mathrm{C}\left(53^{\prime}\right)-\mathrm{H}(53 \mathrm{D})$ & 109.5 \\
\hline $\mathrm{S}\left(3^{\prime}\right)-\mathrm{C}\left(53^{\prime}\right)-\mathrm{H}(53 \mathrm{E})$ & 109.5 \\
\hline $\mathrm{H}(53 \mathrm{D})-\mathrm{C}\left(53^{\prime}\right)-\mathrm{H}(53 \mathrm{E})$ & 109.5 \\
\hline $\mathrm{S}\left(3^{\prime}\right)-\mathrm{C}\left(53^{\prime}\right)-\mathrm{H}(53 \mathrm{~F})$ & 109.5 \\
\hline $\mathrm{H}(53 \mathrm{D})-\mathrm{C}\left(53^{\prime}\right)-\mathrm{H}(53 \mathrm{~F})$ & 109.5 \\
\hline $\mathrm{H}(53 \mathrm{E})-\mathrm{C}\left(53^{\prime}\right)-\mathrm{H}(53 \mathrm{~F})$ & 109.5 \\
\hline $\mathrm{S}\left(3^{\prime}\right)-\mathrm{C}\left(54^{\prime}\right)-\mathrm{H}(54 \mathrm{D})$ & 109.5 \\
\hline $\mathrm{S}\left(3^{\prime}\right)-\mathrm{C}\left(54^{\prime}\right)-\mathrm{H}(54 \mathrm{E})$ & 109.5 \\
\hline $\mathrm{H}(54 \mathrm{D})-\mathrm{C}\left(54^{\prime}\right)-\mathrm{H}(54 \mathrm{E})$ & 109.5 \\
\hline $\mathrm{S}\left(3^{\prime}\right)-\mathrm{C}\left(54^{\prime}\right)-\mathrm{H}(54 \mathrm{~F})$ & 109.5 \\
\hline $\mathrm{H}(54 \mathrm{D})-\mathrm{C}\left(54^{\prime}\right)-\mathrm{H}(54 \mathrm{~F})$ & 109.5 \\
\hline $\mathrm{H}(54 \mathrm{E})-\mathrm{C}\left(54^{\prime}\right)-\mathrm{H}(54 \mathrm{~F})$ & 109.5 \\
\hline $\mathrm{C}(1)-\mathrm{O}(1)-\mathrm{Ce}(1)$ & $92.3(6)$ \\
\hline $\mathrm{C}(1)-\mathrm{O}(2)-\mathrm{Ce}(1)$ & $95.3(6)$ \\
\hline $\mathrm{C}(17)-\mathrm{O}(3)-\mathrm{Ce}(1)$ & $93.2(6)$ \\
\hline $\mathrm{C}(17)-\mathrm{O}(4)-\mathrm{Ce}(1)$ & $95.2(6)$ \\
\hline $\mathrm{C}(33)-\mathrm{O}(5)-\mathrm{Ce}(1)$ & $93.4(5)$ \\
\hline $\mathrm{C}(33)-\mathrm{O}(6)-\mathrm{Ce}(1)$ & $92.4(5)$ \\
\hline $\mathrm{S}(1)-\mathrm{O}(7)-\mathrm{Ce}(1)$ & $141.4(4)$ \\
\hline $\mathrm{S}(2)-\mathrm{O}(8)-\mathrm{Ce}(1)$ & $133.1(4)$ \\
\hline $\mathrm{S}\left(2^{\prime}\right)-\mathrm{O}(8)-\mathrm{Ce}(1)$ & $135.8(4)$ \\
\hline $\mathrm{O}(1)-\mathrm{C}(1)-\mathrm{O}(2)$ & $121.5(9)$ \\
\hline
\end{tabular}




\begin{tabular}{|c|c|}
\hline $\mathrm{O}(1)-\mathrm{C}(1)-\mathrm{C}(2)$ & $120.8(10)$ \\
\hline $\mathrm{O}(2)-\mathrm{C}(1)-\mathrm{C}(2)$ & $117.6(10)$ \\
\hline $\mathrm{O}(1)-\mathrm{C}(1)-\mathrm{Ce}(1)$ & $62.6(5)$ \\
\hline $\mathrm{O}(2)-\mathrm{C}(1)-\mathrm{Ce}(1)$ & $59.4(5)$ \\
\hline $\mathrm{C}(2)-\mathrm{C}(1)-\mathrm{Ce}(1)$ & $170.1(7)$ \\
\hline$C(7)-C(2)-C(3)$ & $119.6(9)$ \\
\hline$C(7)-C(2)-C(1)$ & $123.4(9)$ \\
\hline $\mathrm{C}(3)-\mathrm{C}(2)-\mathrm{C}(1)$ & $117.0(9)$ \\
\hline$C(4)-C(3)-C(2)$ & $120.0(10)$ \\
\hline $\mathrm{C}(4)-\mathrm{C}(3)-\mathrm{H}(3)$ & 120.0 \\
\hline $\mathrm{C}(2)-\mathrm{C}(3)-\mathrm{H}(3)$ & 120.0 \\
\hline$C(5)-C(4)-C(3)$ & $120.0(10)$ \\
\hline $\mathrm{C}(5)-\mathrm{C}(4)-\mathrm{H}(4)$ & 120.0 \\
\hline $\mathrm{C}(3)-\mathrm{C}(4)-\mathrm{H}(4)$ & 120.0 \\
\hline$C(6)-C(5)-C(4)$ & $121.1(10)$ \\
\hline $\mathrm{C}(6)-\mathrm{C}(5)-\mathrm{H}(5)$ & 119.4 \\
\hline $\mathrm{C}(4)-\mathrm{C}(5)-\mathrm{H}(5)$ & 119.4 \\
\hline$C(5)-C(6)-C(7)$ & $119.9(10)$ \\
\hline $\mathrm{C}(5)-\mathrm{C}(6)-\mathrm{H}(6)$ & 120.1 \\
\hline $\mathrm{C}(7)-\mathrm{C}(6)-\mathrm{H}(6)$ & 120.1 \\
\hline$C(2)-C(7)-C(6)$ & $119.4(9)$ \\
\hline $\mathrm{C}(2)-\mathrm{C}(7)-\mathrm{C}(8)$ & $125.1(8)$ \\
\hline $\mathrm{C}(6)-\mathrm{C}(7)-\mathrm{C}(8)$ & 115.3(9) \\
\hline $\mathrm{C}(9)-\mathrm{C}(8)-\mathrm{C}(13)$ & $119.3(10)$ \\
\hline $\mathrm{C}(9)-\mathrm{C}(8)-\mathrm{C}(7)$ & $120.8(9)$ \\
\hline $\mathrm{C}(13)-\mathrm{C}(8)-\mathrm{C}(7)$ & $119.8(10)$ \\
\hline $\mathrm{C}(10)-\mathrm{C}(9)-\mathrm{C}(8)$ & $119.7(10)$ \\
\hline$C(10)-C(9)-C(15)$ & $119.9(11)$ \\
\hline $\mathrm{C}(8)-\mathrm{C}(9)-\mathrm{C}(15)$ & $120.3(10)$ \\
\hline $\mathrm{C}(11)-\mathrm{C}(10)-\mathrm{C}(9)$ & $121.5(12)$ \\
\hline $\mathrm{C}(11)-\mathrm{C}(10)-\mathrm{H}(10)$ & 119.2 \\
\hline $\mathrm{C}(9)-\mathrm{C}(10)-\mathrm{H}(10)$ & 119.2 \\
\hline $\mathrm{C}(12)-\mathrm{C}(11)-\mathrm{C}(10)$ & $117.9(11)$ \\
\hline $\mathrm{C}(12)-\mathrm{C}(11)-\mathrm{C}(16)$ & $121.2(12)$ \\
\hline $\mathrm{C}(10)-\mathrm{C}(11)-\mathrm{C}(16)$ & $120.8(13)$ \\
\hline $\mathrm{C}(11)-\mathrm{C}(12)-\mathrm{C}(13)$ & $123.5(12)$ \\
\hline $\mathrm{C}(11)-\mathrm{C}(12)-\mathrm{H}(12)$ & 118.3 \\
\hline $\mathrm{C}(13)-\mathrm{C}(12)-\mathrm{H}(12)$ & 118.3 \\
\hline
\end{tabular}




\begin{tabular}{|c|c|}
\hline 13$)-C(8)$ & $118.0(11)$ \\
\hline$C(12)-C(13)-C(14)$ & $122.4(11)$ \\
\hline$C(8)-C(13)-C(14)$ & $119.7(10)$ \\
\hline $\mathrm{C}(13)-\mathrm{C}(14)-\mathrm{H}(14 \mathrm{~A})$ & 109.5 \\
\hline $\mathrm{C}(13)-\mathrm{C}(14)-\mathrm{H}(14 \mathrm{~B})$ & 109.5 \\
\hline $\mathrm{H}(14 \mathrm{~A})-\mathrm{C}(14)-\mathrm{H}(14 \mathrm{~B})$ & 109.5 \\
\hline $\mathrm{C}(13)-\mathrm{C}(14)-\mathrm{H}(14 \mathrm{C})$ & 109.5 \\
\hline $\mathrm{H}(14 \mathrm{~A})-\mathrm{C}(14)-\mathrm{H}(14 \mathrm{C})$ & 109.5 \\
\hline $\mathrm{H}(14 \mathrm{~B})-\mathrm{C}(14)-\mathrm{H}(14 \mathrm{C})$ & 109.5 \\
\hline $\mathrm{C}(9)-\mathrm{C}(15)-\mathrm{H}(15 \mathrm{~A})$ & 109.5 \\
\hline $\mathrm{C}(9)-\mathrm{C}(15)-\mathrm{H}(15 \mathrm{~B})$ & 109.5 \\
\hline $\mathrm{H}(15 \mathrm{~A})-\mathrm{C}(15)-\mathrm{H}(15 \mathrm{~B})$ & 109.5 \\
\hline $\mathrm{C}(9)-\mathrm{C}(15)-\mathrm{H}(15 \mathrm{C})$ & 109.5 \\
\hline $\mathrm{H}(15 \mathrm{~A})-\mathrm{C}(15)-\mathrm{H}(15 \mathrm{C})$ & 109.5 \\
\hline $\mathrm{H}(15 \mathrm{~B})-\mathrm{C}(15)-\mathrm{H}(15 \mathrm{C})$ & 109.5 \\
\hline $\mathrm{C}(11)-\mathrm{C}(16)-\mathrm{H}(16 \mathrm{~A})$ & 109.5 \\
\hline $\mathrm{C}(11)-\mathrm{C}(16)-\mathrm{H}(16 \mathrm{~B})$ & 109.5 \\
\hline $\mathrm{H}(16 \mathrm{~A})-\mathrm{C}(16)-\mathrm{H}(16 \mathrm{~B})$ & 109.5 \\
\hline $\mathrm{C}(11)-\mathrm{C}(16)-\mathrm{H}(16 \mathrm{C})$ & 109.5 \\
\hline $\mathrm{H}(16 \mathrm{~A})-\mathrm{C}(16)-\mathrm{H}(16 \mathrm{C})$ & 109.5 \\
\hline $\mathrm{H}(16 \mathrm{~B})-\mathrm{C}(16)-\mathrm{H}(16 \mathrm{C})$ & 109.5 \\
\hline $\mathrm{O}(3)-\mathrm{C}(17)-\mathrm{O}(4)$ & $120.5(9)$ \\
\hline $\mathrm{O}(3)-\mathrm{C}(17)-\mathrm{C}(18)$ & 121.6(9) \\
\hline $\mathrm{O}(4)-\mathrm{C}(17)-\mathrm{C}(18)$ & $117.9(9)$ \\
\hline $\mathrm{O}(3)-\mathrm{C}(17)-\mathrm{Ce}(1)$ & $61.4(5)$ \\
\hline $\mathrm{O}(4)-\mathrm{C}(17)-\mathrm{Ce}(1)$ & $59.2(5)$ \\
\hline $\mathrm{C}(18)-\mathrm{C}(17)-\mathrm{Ce}(1)$ & $175.0(6)$ \\
\hline $\mathrm{C}(23)-\mathrm{C}(18)-\mathrm{C}(19)$ & 117.7(9) \\
\hline $\mathrm{C}(23)-\mathrm{C}(18)-\mathrm{C}(17)$ & $125.0(8)$ \\
\hline $\mathrm{C}(19)-\mathrm{C}(18)-\mathrm{C}(17)$ & 117.2(9) \\
\hline$C(20)-C(19)-C(18)$ & 121.1(9) \\
\hline $\mathrm{C}(20)-\mathrm{C}(19)-\mathrm{H}(19)$ & 119.4 \\
\hline $\mathrm{C}(18)-\mathrm{C}(19)-\mathrm{H}(19)$ & 119.4 \\
\hline$C(19)-C(20)-C(21)$ & $121.0(9)$ \\
\hline $\mathrm{C}(19)-\mathrm{C}(20)-\mathrm{H}(20)$ & 119.5 \\
\hline $\mathrm{C}(21)-\mathrm{C}(20)-\mathrm{H}(20)$ & 119.5 \\
\hline$C(20)-C(21)-C(22)$ & $118.9(9)$ \\
\hline $\mathrm{C}(20)-\mathrm{C}(21)-\mathrm{H}(21)$ & 120.5 \\
\hline
\end{tabular}




$\begin{array}{ll}\mathrm{C}(22)-\mathrm{C}(21)-\mathrm{H}(21) & 120.5 \\ \mathrm{C}(23)-\mathrm{C}(22)-\mathrm{C}(21) & 119.8(9) \\ \mathrm{C}(23)-\mathrm{C}(22)-\mathrm{H}(22) & 120.1 \\ \mathrm{C}(21)-\mathrm{C}(22)-\mathrm{H}(22) & 120.1 \\ \mathrm{C}(18)-\mathrm{C}(23)-\mathrm{C}(22) & 121.3(9) \\ \mathrm{C}(18)-\mathrm{C}(23)-\mathrm{C}(24) & 124.2(9) \\ \mathrm{C}(22)-\mathrm{C}(23)-\mathrm{C}(24) & 114.5(9) \\ \mathrm{C}(29)-\mathrm{C}(24)-\mathrm{C}(25) & 119.0(9) \\ \mathrm{C}(29)-\mathrm{C}(24)-\mathrm{C}(23) & 120.4(8) \\ \mathrm{C}(25)-\mathrm{C}(24)-\mathrm{C}(23) & 120.6(7) \\ \mathrm{C}(26)-\mathrm{C}(25)-\mathrm{C}(24) & 120.7(9) \\ \mathrm{C}(26)-\mathrm{C}(25)-\mathrm{C}(32) & 119.9(8) \\ \mathrm{C}(24)-\mathrm{C}(25)-\mathrm{C}(32) & 119.4(8) \\ \mathrm{C}(25)-\mathrm{C}(26)-\mathrm{C}(27) & 121.2(9) \\ \mathrm{C}(25)-\mathrm{C}(26)-\mathrm{H}(26) & 119.4 \\ \mathrm{C}(27)-\mathrm{C}(26)-\mathrm{H}(26) & 119.4 \\ \mathrm{H}(10 \mathrm{~A})-\mathrm{O}(10)-\mathrm{H}(10 \mathrm{~B}) & 104.5 \\ \mathrm{C}(28)-\mathrm{C}(27)-\mathrm{C}(26) & 117.4(9) \\ \mathrm{C}(28)-\mathrm{C}(27)-\mathrm{C}(31) & 122.9(9) \\ \mathrm{C}(26)-\mathrm{C}(27)-\mathrm{C}(31) & 119.7(9) \\ \mathrm{C}(27)-\mathrm{C}(28)-\mathrm{C}(29) & 123.3(9) \\ \mathrm{C}(27)-\mathrm{C}(28)-\mathrm{H}(28) & 118.4 \\ \mathrm{C}(29)-\mathrm{C}(28)-\mathrm{H}(28) & 118.4 \\ \mathrm{C}(28)-\mathrm{C}(29)-\mathrm{C}(24) & 118.4(9) \\ \mathrm{C}(28)-\mathrm{C}(29)-\mathrm{C}(30) & 121.3(9) \\ \mathrm{C}(24)-\mathrm{C}(29)-\mathrm{C}(30) & 120.3(9) \\ \mathrm{C}(29)-\mathrm{C}(30)-\mathrm{H}(30 \mathrm{~A}) & 109.5 \\ \mathrm{C}(29)-\mathrm{C}(30)-\mathrm{H}(30 \mathrm{~B}) & 109.5 \\ \mathrm{H}(30 \mathrm{~A})-\mathrm{C}(30)-\mathrm{H}(30 \mathrm{~B}) & 109.5 \\ \mathrm{C}(29)-\mathrm{C}(30)-\mathrm{H}(30 \mathrm{C}) & 109.5 \\ \mathrm{H}(30 \mathrm{~A})-\mathrm{C}(30)-\mathrm{H}(30 \mathrm{C}) & 109.5 \\ \mathrm{H}(30 \mathrm{~B})-\mathrm{C}(30)-\mathrm{H}(30 \mathrm{C}) & 109.5 \\ \mathrm{C}(27)-\mathrm{C}(31)-\mathrm{H}(31 \mathrm{~A}) & 109.5 \\ \mathrm{C}(27)-\mathrm{C}(31)-\mathrm{H}(31 \mathrm{~B}) & 109.5 \\ \mathrm{H}(31)-\mathrm{H}(31 \mathrm{~B}) & 109.5 \\ \mathrm{H}(31 \mathrm{C}) & 109.5 \\ 109.5 \\ \mathrm{H}(31 \mathrm{C}) & 109.5 \\ \end{array}$




\begin{tabular}{|c|c|}
\hline $\mathrm{C}(25)-\mathrm{C}(32)-\mathrm{H}(32 \mathrm{~A})$ & 109.5 \\
\hline $\mathrm{C}(25)-\mathrm{C}(32)-\mathrm{H}(32 \mathrm{~B})$ & 109.5 \\
\hline $\mathrm{H}(32 \mathrm{~A})-\mathrm{C}(32)-\mathrm{H}(32 \mathrm{~B})$ & 109.5 \\
\hline $\mathrm{C}(25)-\mathrm{C}(32)-\mathrm{H}(32 \mathrm{C})$ & 109.5 \\
\hline $\mathrm{H}(32 \mathrm{~A})-\mathrm{C}(32)-\mathrm{H}(32 \mathrm{C})$ & 109.5 \\
\hline $\mathrm{H}(32 \mathrm{~B})-\mathrm{C}(32)-\mathrm{H}(32 \mathrm{C})$ & 109.5 \\
\hline $\mathrm{O}(5)-\mathrm{C}(33)-\mathrm{O}(6)$ & $122.7(8)$ \\
\hline $\mathrm{O}(5)-\mathrm{C}(33)-\mathrm{C}(34)$ & $116.6(8)$ \\
\hline $\mathrm{O}(6)-\mathrm{C}(33)-\mathrm{C}(34)$ & $120.6(8)$ \\
\hline $\mathrm{O}(5)-\mathrm{C}(33)-\mathrm{Ce}(1)$ & $61.4(4)$ \\
\hline $\mathrm{O}(6)-\mathrm{C}(33)-\mathrm{Ce}(1)$ & $62.2(4)$ \\
\hline $\mathrm{C}(34)-\mathrm{C}(33)-\mathrm{Ce}(1)$ & $168.5(6)$ \\
\hline $\mathrm{C}(35)-\mathrm{C}(34)-\mathrm{C}(39)$ & $118.5(8)$ \\
\hline $\mathrm{C}(35)-\mathrm{C}(34)-\mathrm{C}(33)$ & $118.4(8)$ \\
\hline $\mathrm{C}(39)-\mathrm{C}(34)-\mathrm{C}(33)$ & $123.2(8)$ \\
\hline$C(36)-C(35)-C(34)$ & 123.1(9) \\
\hline $\mathrm{C}(36)-\mathrm{C}(35)-\mathrm{H}(35)$ & 118.5 \\
\hline $\mathrm{C}(34)-\mathrm{C}(35)-\mathrm{H}(35)$ & 118.5 \\
\hline $\mathrm{C}(35)-\mathrm{C}(36)-\mathrm{C}(37)$ & $118.9(10)$ \\
\hline $\mathrm{C}(35)-\mathrm{C}(36)-\mathrm{H}(36)$ & 120.6 \\
\hline $\mathrm{C}(37)-\mathrm{C}(36)-\mathrm{H}(36)$ & 120.6 \\
\hline $\mathrm{C}(38)-\mathrm{C}(37)-\mathrm{C}(36)$ & $119.8(9)$ \\
\hline $\mathrm{C}(38)-\mathrm{C}(37)-\mathrm{H}(37)$ & 120.1 \\
\hline $\mathrm{C}(36)-\mathrm{C}(37)-\mathrm{H}(37)$ & 120.1 \\
\hline $\mathrm{C}(37)-\mathrm{C}(38)-\mathrm{C}(39)$ & $120.9(9)$ \\
\hline $\mathrm{C}(37)-\mathrm{C}(38)-\mathrm{H}(38)$ & 119.5 \\
\hline $\mathrm{C}(39)-\mathrm{C}(38)-\mathrm{H}(38)$ & 119.5 \\
\hline $\mathrm{C}(34)-\mathrm{C}(39)-\mathrm{C}(38)$ & 118.8(9) \\
\hline $\mathrm{C}(34)-\mathrm{C}(39)-\mathrm{C}(40)$ & $124.2(8)$ \\
\hline $\mathrm{C}(38)-\mathrm{C}(39)-\mathrm{C}(40)$ & $116.9(8)$ \\
\hline $\mathrm{C}(45)-\mathrm{C}(40)-\mathrm{C}(41)$ & 119.4(9) \\
\hline$C(45)-C(40)-C(39)$ & $120.5(9)$ \\
\hline $\mathrm{C}(41)-\mathrm{C}(40)-\mathrm{C}(39)$ & $120.1(10)$ \\
\hline $\mathrm{C}(42)-\mathrm{C}(41)-\mathrm{C}(40)$ & $118.3(10)$ \\
\hline $\mathrm{C}(42)-\mathrm{C}(41)-\mathrm{C}(47)$ & $121.6(11)$ \\
\hline $\mathrm{C}(40)-\mathrm{C}(41)-\mathrm{C}(47)$ & $120.0(10)$ \\
\hline$C(41)-C(42)-C(43)$ & $123.1(11)$ \\
\hline $\mathrm{C}(41)-\mathrm{C}(42)-\mathrm{H}(42)$ & 118.4 \\
\hline
\end{tabular}




\begin{tabular}{|c|c|}
\hline $\mathrm{C}(43)-\mathrm{C}$ & 118.4 \\
\hline$C(42)-C(43)-C(44)$ & $118.1(10)$ \\
\hline $\mathrm{C}(42)-\mathrm{C}(43)-\mathrm{C}(48)$ & $122.8(12)$ \\
\hline$C(44)-C(43)-C(48)$ & $119.1(12)$ \\
\hline $\mathrm{C}(43)-\mathrm{C}(44)-\mathrm{C}(45)$ & $121.0(11)$ \\
\hline $\mathrm{C}(43)-\mathrm{C}(44)-\mathrm{H}(44)$ & 119.5 \\
\hline $\mathrm{C}(45)-\mathrm{C}(44)-\mathrm{H}(44)$ & 119.5 \\
\hline$C(40)-C(45)-C(44)$ & $120.0(10)$ \\
\hline $\mathrm{C}(40)-\mathrm{C}(45)-\mathrm{C}(46)$ & $121.4(9)$ \\
\hline $\mathrm{C}(44)-\mathrm{C}(45)-\mathrm{C}(46)$ & $118.6(10)$ \\
\hline $\mathrm{C}(45)-\mathrm{C}(46)-\mathrm{H}(46 \mathrm{~A})$ & 109.5 \\
\hline $\mathrm{C}(45)-\mathrm{C}(46)-\mathrm{H}(46 \mathrm{~B})$ & 109.5 \\
\hline $\mathrm{H}(46 \mathrm{~A})-\mathrm{C}(46)-\mathrm{H}(46 \mathrm{~B})$ & 109.5 \\
\hline $\mathrm{C}(45)-\mathrm{C}(46)-\mathrm{H}(46 \mathrm{C})$ & 109.5 \\
\hline $\mathrm{H}(46 \mathrm{~A})-\mathrm{C}(46)-\mathrm{H}(46 \mathrm{C})$ & 109.5 \\
\hline $\mathrm{H}(46 \mathrm{~B})-\mathrm{C}(46)-\mathrm{H}(46 \mathrm{C})$ & 109.5 \\
\hline $\mathrm{C}(41)-\mathrm{C}(47)-\mathrm{H}(47 \mathrm{~A})$ & 109.5 \\
\hline $\mathrm{C}(41)-\mathrm{C}(47)-\mathrm{H}(47 \mathrm{~B})$ & 109.5 \\
\hline $\mathrm{H}(47 \mathrm{~A})-\mathrm{C}(47)-\mathrm{H}(47 \mathrm{~B})$ & 109.5 \\
\hline $\mathrm{C}(41)-\mathrm{C}(47)-\mathrm{H}(47 \mathrm{C})$ & 109.5 \\
\hline $\mathrm{H}(47 \mathrm{~A})-\mathrm{C}(47)-\mathrm{H}(47 \mathrm{C})$ & 109.5 \\
\hline $\mathrm{H}(47 \mathrm{~B})-\mathrm{C}(47)-\mathrm{H}(47 \mathrm{C})$ & 109.5 \\
\hline $\mathrm{C}(43)-\mathrm{C}(48)-\mathrm{H}(48 \mathrm{~A})$ & 109.5 \\
\hline $\mathrm{C}(43)-\mathrm{C}(48)-\mathrm{H}(48 \mathrm{~B})$ & 109.5 \\
\hline $\mathrm{H}(48 \mathrm{~A})-\mathrm{C}(48)-\mathrm{H}(48 \mathrm{~B})$ & 109.5 \\
\hline $\mathrm{C}(43)-\mathrm{C}(48)-\mathrm{H}(48 \mathrm{C})$ & 109.5 \\
\hline $\mathrm{H}(48 \mathrm{~A})-\mathrm{C}(48)-\mathrm{H}(48 \mathrm{C})$ & 109.5 \\
\hline $\mathrm{H}(48 \mathrm{~B})-\mathrm{C}(48)-\mathrm{H}(48 \mathrm{C})$ & 109.5 \\
\hline $\mathrm{S}(1)-\mathrm{C}(49)-\mathrm{H}(49 \mathrm{~A})$ & 109.5 \\
\hline $\mathrm{S}(1)-\mathrm{C}(49)-\mathrm{H}(49 \mathrm{~B})$ & 109.5 \\
\hline $\mathrm{H}(49 \mathrm{~A})-\mathrm{C}(49)-\mathrm{H}(49 \mathrm{~B})$ & 109.5 \\
\hline $\mathrm{S}(1)-\mathrm{C}(49)-\mathrm{H}(49 \mathrm{C})$ & 109.5 \\
\hline $\mathrm{H}(49 \mathrm{~A})-\mathrm{C}(49)-\mathrm{H}(49 \mathrm{C})$ & 109.5 \\
\hline H(49B)-C(49)-H(49C) & 109.5 \\
\hline $\mathrm{S}(1)-\mathrm{C}(50)-\mathrm{H}(50 \mathrm{~A})$ & 109.5 \\
\hline $\mathrm{S}(1)-\mathrm{C}(50)-\mathrm{H}(50 \mathrm{~B})$ & 109.5 \\
\hline $\mathrm{H}(50 \mathrm{~A})-\mathrm{C}(50)-\mathrm{H}(50 \mathrm{~B})$ & 109.5 \\
\hline $\mathrm{S}(1)-\mathrm{C}(50)-\mathrm{H}(50 \mathrm{C})$ & 109.5 \\
\hline
\end{tabular}


$\mathrm{H}(50 \mathrm{~A})-\mathrm{C}(50)-\mathrm{H}(50 \mathrm{C}) \quad 109.5$

$\mathrm{H}(50 \mathrm{~B})-\mathrm{C}(50)-\mathrm{H}(50 \mathrm{C}) \quad 109.5$

Symmetry transformations used to generate equivalent atoms: 
Table S9. Anisotropic displacement parameters $\left(\AA^{2} \times 10^{3}\right)$ for complex 56.

The anisotropic displacement factor exponent takes the form: $\quad-2 \pi^{2}\left[h^{2} a^{* 2} U^{11}+\ldots\right.$ $+2 \mathrm{~h} \mathrm{k} \mathrm{a}^{*} \mathrm{~b}^{*} \mathrm{U}^{12}$ ]
U11
$\mathrm{U}^{22}$
$\mathrm{U}^{33}$
$\mathrm{U}^{23}$
U13
$\mathrm{U}^{12}$

\begin{tabular}{|c|c|c|c|c|c|c|}
\hline $\mathrm{Ce}(1)$ & $38(1)$ & $29(1)$ & $51(1)$ & $3(1)$ & $23(1)$ & $1(1)$ \\
\hline$S(1)$ & $64(2)$ & $57(2)$ & 107(3) & $6(2)$ & $32(2)$ & $29(2)$ \\
\hline$S(2)$ & $49(3)$ & $79(4)$ & $45(3)$ & $0(3)$ & $19(2)$ & $-28(3)$ \\
\hline$S(3)$ & $63(3)$ & $31(3)$ & $42(3)$ & $3(2)$ & $8(3)$ & $4(2)$ \\
\hline $\mathrm{O}(9)$ & $33(7)$ & $23(6)$ & $35(7)$ & $5(5)$ & $6(6)$ & $6(5)$ \\
\hline$C(51)$ & $34(13)$ & $100(20)$ & $82(16)$ & $-5(14)$ & $24(13)$ & $-23(13)$ \\
\hline $\mathrm{C}(52)$ & $58(19)$ & 67(19) & $80(20)$ & $-29(14)$ & $-2(15)$ & $-30(14)$ \\
\hline $\mathrm{C}(53)$ & $48(17)$ & $64(19)$ & $92(19)$ & $-9(13)$ & $23(13)$ & $10(14)$ \\
\hline $\mathrm{C}(54)$ & 131(16) & $25(11)$ & $83(14)$ & $8(10)$ & $36(12)$ & 21(11) \\
\hline $\mathrm{S}\left(2^{\prime}\right)$ & $47(3)$ & $33(3)$ & $41(3)$ & $1(2)$ & $12(2)$ & $-6(2)$ \\
\hline$S\left(3^{\prime}\right)$ & $50(3)$ & $30(2)$ & $39(3)$ & $1(2)$ & $9(2)$ & $7(2)$ \\
\hline $\mathrm{O}\left(9^{\prime}\right)$ & $35(7)$ & $31(7)$ & $37(7)$ & $-5(6)$ & $1(6)$ & $10(6)$ \\
\hline $\mathrm{C}\left(51^{\prime}\right)$ & $31(13)$ & $56(12)$ & $69(14)$ & $-3(13)$ & 19(11) & $-11(12)$ \\
\hline $\mathrm{C}\left(52^{\prime}\right)$ & $52(17)$ & $62(17)$ & $57(14)$ & $-24(13)$ & $-1(14)$ & $-18(13)$ \\
\hline$C\left(53^{\prime}\right)$ & $24(11)$ & $42(13)$ & $61(14)$ & $-20(10)$ & $9(10)$ & $-19(10)$ \\
\hline $\mathrm{C}\left(54^{\prime}\right)$ & $67(12)$ & $58(11)$ & $89(13)$ & $26(12)$ & $48(10)$ & $4(11)$ \\
\hline $\mathrm{O}(1)$ & $36(4)$ & $79(5)$ & $61(5)$ & $31(4)$ & $14(4)$ & $-10(4)$ \\
\hline $\mathrm{O}(2)$ & $63(5)$ & $73(5)$ & $71(5)$ & $-21(4)$ & $46(4)$ & $-35(4)$ \\
\hline $\mathrm{O}(3)$ & $59(4)$ & $27(3)$ & $62(4)$ & $-4(4)$ & $26(3)$ & $0(4)$ \\
\hline $\mathrm{O}(4)$ & $55(4)$ & $42(4)$ & $62(5)$ & $-7(4)$ & $-1(4)$ & $14(4)$ \\
\hline $\mathrm{O}(5)$ & $38(4)$ & $30(3)$ & $66(4)$ & $5(3)$ & $33(3)$ & $3(3)$ \\
\hline $\mathrm{O}(6)$ & $37(3)$ & $35(3)$ & $69(4)$ & $14(4)$ & $26(3)$ & $5(3)$ \\
\hline $\mathrm{O}(7)$ & $41(4)$ & $37(4)$ & $85(5)$ & $3(4)$ & $27(4)$ & $10(3)$ \\
\hline $\mathrm{O}(8)$ & $48(4)$ & $49(4)$ & $44(4)$ & $-3(3)$ & $15(3)$ & $-5(3)$ \\
\hline $\mathrm{C}(1)$ & $36(6)$ & $35(6)$ & $82(8)$ & $8(6)$ & $30(6)$ & $8(5)$ \\
\hline $\mathrm{C}(2)$ & $37(6)$ & $42(6)$ & $67(7)$ & $5(5)$ & $26(5)$ & $0(5)$ \\
\hline $\mathrm{C}(3)$ & $52(7)$ & $45(6)$ & $78(8)$ & $10(6)$ & $33(6)$ & $8(5)$ \\
\hline
\end{tabular}




\begin{tabular}{|c|c|c|c|c|c|c|}
\hline $\mathrm{C}(4)$ & $57(7)$ & $46(6)$ & $86(8)$ & $19(6)$ & $43(6)$ & $9(5)$ \\
\hline$C(5)$ & $52(7)$ & $54(7)$ & $89(9)$ & $1(6)$ & $45(6)$ & $-12(5)$ \\
\hline$C(6)$ & $39(6)$ & $66(7)$ & $63(7)$ & $-3(6)$ & $22(5)$ & $-14(6)$ \\
\hline$C(7)$ & $42(6)$ & $44(6)$ & $58(6)$ & $3(5)$ & $31(5)$ & $-6(5)$ \\
\hline$C(8)$ & $30(5)$ & $53(6)$ & $72(8)$ & $4(6)$ & $29(5)$ & $9(5)$ \\
\hline $\mathrm{C}(9)$ & $32(5)$ & $55(7)$ & $67(7)$ & $7(6)$ & $23(5)$ & $-5(5)$ \\
\hline$C(10)$ & $34(6)$ & $85(9)$ & $66(8)$ & $7(7)$ & $24(6)$ & $-1(6)$ \\
\hline $\mathrm{C}(11)$ & $34(6)$ & $80(8)$ & $75(9)$ & $24(7)$ & $28(6)$ & $22(6)$ \\
\hline$C(12)$ & $49(7)$ & $59(7)$ & $91(9)$ & $17(7)$ & $33(7)$ & $13(6)$ \\
\hline$C(13)$ & $58(6)$ & $56(7)$ & $69(7)$ & $12(6)$ & $33(6)$ & $17(6)$ \\
\hline$C(14)$ & $118(12)$ & $60(8)$ & $82(9)$ & $-1(7)$ & $45(9)$ & $29(8)$ \\
\hline$C(15)$ & $61(7)$ & $54(7)$ & $81(8)$ & $0(7)$ & $22(6)$ & $-2(6)$ \\
\hline$C(16)$ & $77(9)$ & $97(11)$ & $106(11)$ & $39(9)$ & $27(8)$ & $20(8)$ \\
\hline $\mathrm{C}(17)$ & $24(5)$ & $47(6)$ & $58(7)$ & $-3(5)$ & $13(5)$ & $-2(5)$ \\
\hline$C(18)$ & $30(5)$ & $26(5)$ & $64(7)$ & $-10(5)$ & $24(5)$ & $1(4)$ \\
\hline$C(19)$ & $44(6)$ & $34(5)$ & $55(6)$ & $-3(5)$ & $18(5)$ & $6(4)$ \\
\hline$C(20)$ & $49(6)$ & $40(6)$ & $69(7)$ & $-9(6)$ & $25(6)$ & $6(5)$ \\
\hline $\mathrm{C}(21)$ & $55(6)$ & $34(5)$ & $60(7)$ & $-11(5)$ & $31(6)$ & $-7(5)$ \\
\hline$C(22)$ & $45(6)$ & $38(5)$ & $50(6)$ & $2(5)$ & $24(5)$ & $0(5)$ \\
\hline$C(23)$ & $28(5)$ & $34(5)$ & $60(7)$ & $-2(5)$ & $22(5)$ & $-7(4)$ \\
\hline$C(24)$ & $37(5)$ & $30(5)$ & $54(5)$ & $-9(5)$ & $16(4)$ & $-4(5)$ \\
\hline$C(25)$ & $34(5)$ & $30(5)$ & $54(6)$ & $-2(5)$ & $13(5)$ & $-6(4)$ \\
\hline$C(26)$ & $61(7)$ & $26(5)$ & $63(7)$ & $6(5)$ & $14(6)$ & $7(5)$ \\
\hline $\mathrm{O}(10)$ & $78(9)$ & $54(7)$ & $62(7)$ & 0 & $16(7)$ & 0 \\
\hline$C(27)$ & $34(6)$ & $41(6)$ & $67(7)$ & $0(5)$ & $9(5)$ & $3(5)$ \\
\hline $\mathrm{C}(28)$ & $28(5)$ & $39(6)$ & $106(9)$ & $3(6)$ & $17(6)$ & $6(5)$ \\
\hline$C(29)$ & $37(5)$ & $27(6)$ & $86(8)$ & $-2(5)$ & $18(5)$ & $-1(4)$ \\
\hline$C(30)$ & $52(7)$ & $35(6)$ & $140(12)$ & $11(7)$ & $49(7)$ & $1(5)$ \\
\hline $\mathrm{C}(31)$ & $50(7)$ & $38(6)$ & 131(11) & $-3(7)$ & $8(7)$ & $21(5)$ \\
\hline$C(32)$ & $47(6)$ & $38(6)$ & $108(9)$ & $-12(6)$ & $45(6)$ & $-6(5)$ \\
\hline$C(33)$ & $30(5)$ & $38(5)$ & $36(5)$ & $-4(4)$ & $10(4)$ & $-10(4)$ \\
\hline$C(34)$ & $26(5)$ & $39(5)$ & $38(5)$ & $2(4)$ & $11(4)$ & $-5(4)$ \\
\hline$C(35)$ & $34(5)$ & $45(6)$ & $52(6)$ & $11(5)$ & $14(5)$ & $-4(5)$ \\
\hline$C(36)$ & $46(6)$ & $67(7)$ & $62(7)$ & $25(6)$ & $10(5)$ & $-2(6)$ \\
\hline$C(37)$ & $46(6)$ & $83(9)$ & $61(7)$ & $30(7)$ & $23(6)$ & $-8(6)$ \\
\hline $\mathrm{C}(38)$ & $33(6)$ & $85(8)$ & $57(7)$ & $22(6)$ & $16(5)$ & $-9(6)$ \\
\hline C(39) & $37(5)$ & $50(6)$ & $42(6)$ & $12(5)$ & $17(5)$ & $5(5)$ \\
\hline$C(40)$ & $23(5)$ & $34(5)$ & $59(7)$ & $10(4)$ & $14(5)$ & $3(4)$ \\
\hline
\end{tabular}




$\begin{array}{lcccccc}\mathrm{C}(41) & 53(7) & 51(6) & 53(7) & 1(6) & 24(6) & 1(6) \\ \mathrm{C}(42) & 41(6) & 50(7) & 100(10) & 16(7) & 36(7) & 9(5) \\ \mathrm{C}(43) & 38(6) & 62(8) & 76(8) & 34(7) & 28(6) & 15(6) \\ \mathrm{C}(44) & 30(5) & 89(8) & 50(6) & 23(7) & 6(4) & -9(7) \\ \mathrm{C}(45) & 20(5) & 56(6) & 61(7) & 5(6) & 9(5) & -2(5) \\ \mathrm{C}(46) & 61(7) & 49(7) & 78(8) & -10(6) & 15(6) & 10(6) \\ \mathrm{C}(47) & 79(9) & 59(8) & 88(10) & -6(7) & 40(8) & -5(7) \\ \mathrm{C}(48) & 62(8) & 103(11) & 120(11) & 72(10) & 30(8) & 14(8) \\ \mathrm{C}(49) & 45(7) & 74(8) & 108(10) & 8(8) & 31(7) & 5(6) \\ \mathrm{C}(50) & 93(11) & 91(11) & 146(14) & 67(10) & 4(10) & -40(9) \\ \end{array}$


Table S10. Hydrogen coordinates ( x 104) and isotropic displacement parameters $\left(\AA^{2} \times 10^{3}\right)$ for complex $\mathbf{5 6}$.

\begin{tabular}{|c|c|c|c|c|}
\hline & $\mathrm{x}$ & $\mathrm{y}$ & $\mathrm{z}$ & $\mathrm{U}(\mathrm{eq})$ \\
\hline $\mathrm{H}(51 \mathrm{~A})$ & 4114 & 3550 & 2861 & 105 \\
\hline $\mathrm{H}(51 \mathrm{~B})$ & 3789 & 2712 & 2541 & 105 \\
\hline $\mathrm{H}(51 \mathrm{C})$ & 4268 & 3389 & 2532 & 105 \\
\hline $\mathrm{H}(52 \mathrm{~A})$ & 4131 & 715 & 2236 & 111 \\
\hline $\mathrm{H}(52 \mathrm{~B})$ & 4370 & -873 & 2397 & 111 \\
\hline $\mathrm{H}(52 \mathrm{C})$ & 4672 & 609 & 2366 & 111 \\
\hline $\mathrm{H}(53 \mathrm{~A})$ & 4112 & 5890 & 3211 & 101 \\
\hline $\mathrm{H}(53 \mathrm{~B})$ & 4228 & 7192 & 3496 & 101 \\
\hline $\mathrm{H}(53 \mathrm{C})$ & 4355 & 5401 & 3587 & 101 \\
\hline $\mathrm{H}(54 \mathrm{~A})$ & 4906 & 8480 & 2974 & 118 \\
\hline $\mathrm{H}(54 \mathrm{~B})$ & 4411 & 8396 & 3022 & 118 \\
\hline $\mathrm{H}(54 \mathrm{C})$ & 4562 & 7134 & 2795 & 118 \\
\hline $\mathrm{H}(51 \mathrm{D})$ & 3841 & 1499 & 2687 & 77 \\
\hline $\mathrm{H}(51 \mathrm{E})$ & 3792 & 3035 & 2463 & 77 \\
\hline $\mathrm{H}(51 \mathrm{~F})$ & 4033 & 3136 & 2853 & 77 \\
\hline $\mathrm{H}(52 \mathrm{D})$ & 4516 & -575 & 2422 & 91 \\
\hline $\mathrm{H}(52 \mathrm{E})$ & 4310 & 511 & 2106 & 91 \\
\hline $\mathrm{H}(52 \mathrm{~F})$ & 4011 & 83 & 2346 & 91 \\
\hline $\mathrm{H}(53 \mathrm{D})$ & 4151 & 6003 & 3432 & 65 \\
\hline $\mathrm{H}(53 \mathrm{E})$ & 4421 & 7622 & 3511 & 65 \\
\hline $\mathrm{H}(53 \mathrm{~F})$ & 4670 & 6017 & 3650 & 65 \\
\hline $\mathrm{H}(54 \mathrm{D})$ & 5339 & 7075 & 3429 & 99 \\
\hline $\mathrm{H}(54 \mathrm{E})$ & 5053 & 8579 & 3265 & 99 \\
\hline $\mathrm{H}(54 \mathrm{~F})$ & 5294 & 7521 & 3053 & 99 \\
\hline $\mathrm{H}(3)$ & 5879 & 6017 & 2809 & 66 \\
\hline $\mathrm{H}(4)$ & 6358 & 7702 & 2632 & 70 \\
\hline $\mathrm{H}(5)$ & 7128 & 7655 & 2887 & 71 \\
\hline $\mathrm{H}(6)$ & 7436 & 5993 & 3325 & 65 \\
\hline
\end{tabular}




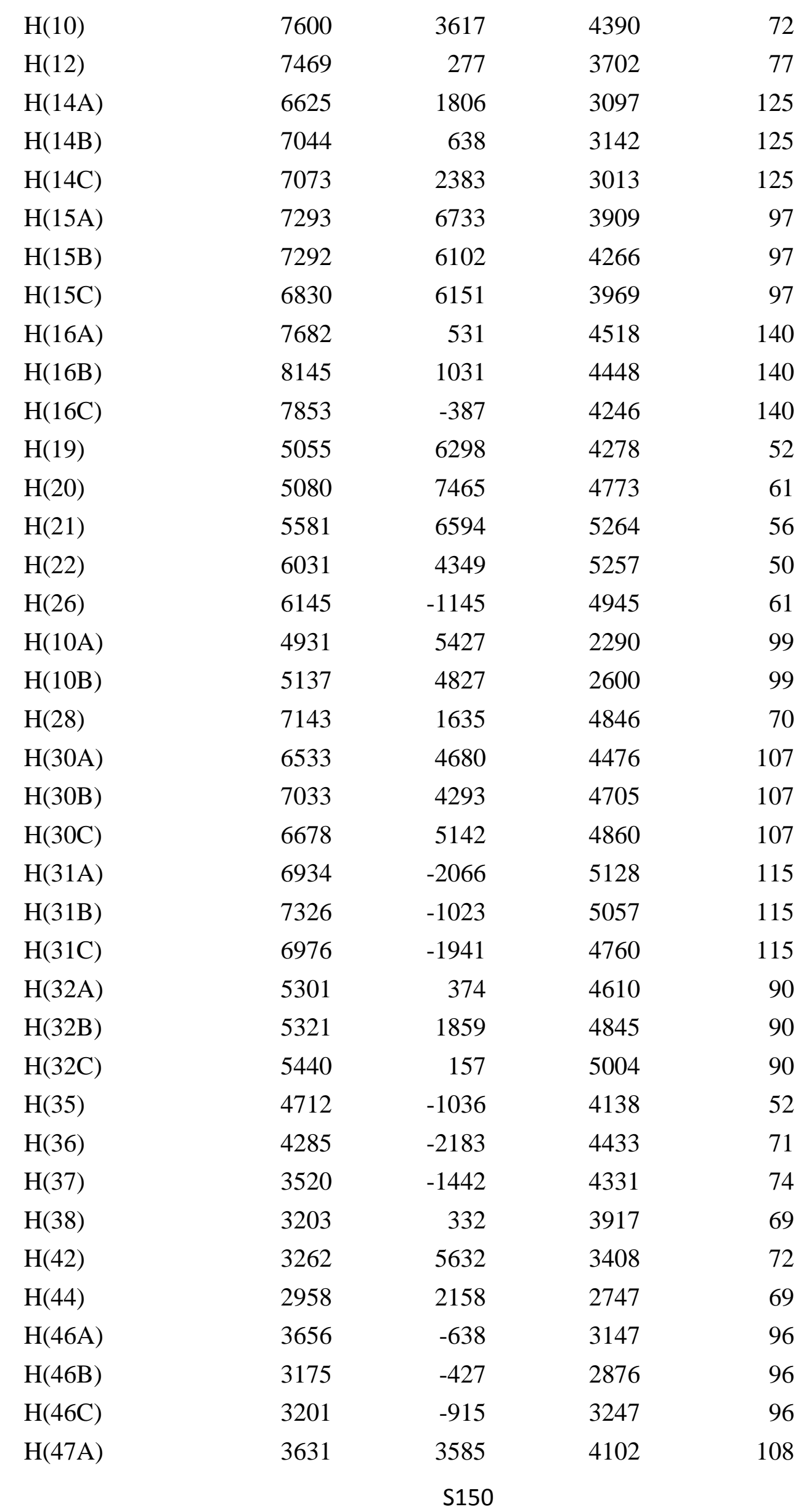




\begin{tabular}{llrll}
$\mathrm{H}(47 \mathrm{~B})$ & 3693 & 5274 & 3958 & 108 \\
$\mathrm{H}(47 \mathrm{C})$ & 4080 & 3979 & 4000 & 108 \\
$\mathrm{H}(48 \mathrm{~A})$ & 2897 & 4835 & 2551 & 141 \\
$\mathrm{H}(48 \mathrm{~B})$ & 2991 & 6246 & 2811 & 141 \\
$\mathrm{H}(48 \mathrm{C})$ & 2535 & 5247 & 2743 & 141 \\
$\mathrm{H}(49 \mathrm{~A})$ & 6566 & 1500 & 3920 & 111 \\
$\mathrm{H}(49 B)$ & 6730 & 108 & 4181 & 111 \\
$\mathrm{H}(49 \mathrm{C})$ & 6280 & 1065 & 4169 & 111 \\
$\mathrm{H}(50 \mathrm{~A})$ & 5673 & -840 & 4092 & 175 \\
$\mathrm{H}(50 B)$ & 6102 & -1963 & 4227 & 175 \\
$\mathrm{H}(50 \mathrm{C})$ & 5687 & -2500 & 3920 & 175 \\
& & & & \\
\hline
\end{tabular}


Table S11. Torsion angles $\left[{ }^{\circ}\right]$ for complex 56.

\begin{tabular}{|c|c|}
\hline $\mathrm{C}(53)-\mathrm{S}(3)-\mathrm{O}(9)-\mathrm{Ce}(1)$ & $-85.6(17)$ \\
\hline $\mathrm{C}(54)-\mathrm{S}(3)-\mathrm{O}(9)-\mathrm{Ce}(1)$ & $176.5(12)$ \\
\hline $\mathrm{C}\left(53^{\prime}\right)-\mathrm{S}\left(3^{\prime}\right)-\mathrm{O}\left(9^{\prime}\right)-\mathrm{Ce}(1)$ & $-46.9(18)$ \\
\hline $\mathrm{C}\left(54^{\prime}\right)-\mathrm{S}\left(3^{\prime}\right)-\mathrm{O}\left(9^{\prime}\right)-\mathrm{Ce}(1)$ & $54.5(17)$ \\
\hline $\mathrm{C}(49)-\mathrm{S}(1)-\mathrm{O}(7)-\mathrm{Ce}(1)$ & $-32.5(8)$ \\
\hline $\mathrm{C}(50)-\mathrm{S}(1)-\mathrm{O}(7)-\mathrm{Ce}(1)$ & $67.9(8)$ \\
\hline $\mathrm{C}(52)-\mathrm{S}(2)-\mathrm{O}(8)-\mathrm{S}\left(2^{\prime}\right)$ & $52.0(17)$ \\
\hline $\mathrm{C}(51)-\mathrm{S}(2)-\mathrm{O}(8)-\mathrm{S}\left(2^{\prime}\right)$ & $-44.9(13)$ \\
\hline $\mathrm{C}(52)-\mathrm{S}(2)-\mathrm{O}(8)-\mathrm{Ce}(1)$ & $179.1(17)$ \\
\hline $\mathrm{C}(51)-\mathrm{S}(2)-\mathrm{O}(8)-\mathrm{Ce}(1)$ & $82.2(14)$ \\
\hline $\mathrm{C}\left(51^{\prime}\right)-\mathrm{S}\left(2^{\prime}\right)-\mathrm{O}(8)-\mathrm{S}(2)$ & $40.0(11)$ \\
\hline $\mathrm{C}\left(52^{\prime}\right)-\mathrm{S}\left(2^{\prime}\right)-\mathrm{O}(8)-\mathrm{S}(2)$ & $-56.8(16)$ \\
\hline $\mathrm{C}\left(51^{\prime}\right)-\mathrm{S}\left(2^{\prime}\right)-\mathrm{O}(8)-\mathrm{Ce}(1)$ & $-83.4(11)$ \\
\hline $\mathrm{C}\left(52^{\prime}\right)-\mathrm{S}\left(2^{\prime}\right)-\mathrm{O}(8)-\mathrm{Ce}(1)$ & $179.8(16)$ \\
\hline $\mathrm{Ce}(1)-\mathrm{O}(1)-\mathrm{C}(1)-\mathrm{O}(2)$ & $-8.0(10)$ \\
\hline $\mathrm{Ce}(1)-\mathrm{O}(1)-\mathrm{C}(1)-\mathrm{C}(2)$ & $169.3(8)$ \\
\hline $\mathrm{Ce}(1)-\mathrm{O}(2)-\mathrm{C}(1)-\mathrm{O}(1)$ & $8.3(10)$ \\
\hline $\mathrm{Ce}(1)-\mathrm{O}(2)-\mathrm{C}(1)-\mathrm{C}(2)$ & $-169.1(7)$ \\
\hline $\mathrm{O}(1)-\mathrm{C}(1)-\mathrm{C}(2)-\mathrm{C}(7)$ & $42.8(14)$ \\
\hline $\mathrm{O}(2)-\mathrm{C}(1)-\mathrm{C}(2)-\mathrm{C}(7)$ & $-139.8(10)$ \\
\hline $\mathrm{O}(1)-\mathrm{C}(1)-\mathrm{C}(2)-\mathrm{C}(3)$ & $-135.6(10)$ \\
\hline $\mathrm{O}(2)-\mathrm{C}(1)-\mathrm{C}(2)-\mathrm{C}(3)$ & $41.8(13)$ \\
\hline $\mathrm{C}(7)-\mathrm{C}(2)-\mathrm{C}(3)-\mathrm{C}(4)$ & $-1.6(15)$ \\
\hline$C(1)-C(2)-C(3)-C(4)$ & $176.8(9)$ \\
\hline $\mathrm{C}(2)-\mathrm{C}(3)-\mathrm{C}(4)-\mathrm{C}(5)$ & $1.1(16)$ \\
\hline$C(3)-C(4)-C(5)-C(6)$ & $-0.5(17)$ \\
\hline$C(4)-C(5)-C(6)-C(7)$ & $0.4(17)$ \\
\hline$C(3)-C(2)-C(7)-C(6)$ & $1.6(15)$ \\
\hline $\mathrm{C}(1)-\mathrm{C}(2)-\mathrm{C}(7)-\mathrm{C}(6)$ & $-176.8(9)$ \\
\hline$C(3)-C(2)-C(7)-C(8)$ & $-173.8(10)$ \\
\hline $\mathrm{C}(1)-\mathrm{C}(2)-\mathrm{C}(7)-\mathrm{C}(8)$ & $7.9(16)$ \\
\hline$C(5)-C(6)-C(7)-C(2)$ & $-1.0(16)$ \\
\hline$C(5)-C(6)-C(7)-C(8)$ & $174.8(10)$ \\
\hline$C(2)-C(7)-C(8)-C(9)$ & $-106.7(12)$ \\
\hline $\mathrm{C}(6)-\mathrm{C}(7)-\mathrm{C}(8)-\mathrm{C}(9)$ & $77.8(12)$ \\
\hline$C(2)-C(7)-C(8)-C(13)$ & $76.6(13)$ \\
\hline
\end{tabular}




\begin{tabular}{|c|c|}
\hline$C(6)-C(7)-C(8)-C(13)$ & $-98.9(11)$ \\
\hline $\mathrm{C}(13)-\mathrm{C}(8)-\mathrm{C}(9)-\mathrm{C}(10)$ & $0.1(14)$ \\
\hline $\mathrm{C}(7)-\mathrm{C}(8)-\mathrm{C}(9)-\mathrm{C}(10)$ & $-176.6(8)$ \\
\hline$C(13)-C(8)-C(9)-C(15)$ & $-177.7(9)$ \\
\hline$C(7)-C(8)-C(9)-C(15)$ & $5.5(14)$ \\
\hline$C(8)-C(9)-C(10)-C(11)$ & $1.9(15)$ \\
\hline $\mathrm{C}(15)-\mathrm{C}(9)-\mathrm{C}(10)-\mathrm{C}(11)$ & $179.8(9)$ \\
\hline$C(9)-C(10)-C(11)-C(12)$ & $-1.8(15)$ \\
\hline$C(9)-C(10)-C(11)-C(16)$ & $-178.9(10)$ \\
\hline$C(10)-C(11)-C(12)-C(13)$ & $-0.4(16)$ \\
\hline $\mathrm{C}(16)-\mathrm{C}(11)-\mathrm{C}(12)-\mathrm{C}(13)$ & $176.6(10)$ \\
\hline $\mathrm{C}(11)-\mathrm{C}(12)-\mathrm{C}(13)-\mathrm{C}(8)$ & $2.4(16)$ \\
\hline $\mathrm{C}(11)-\mathrm{C}(12)-\mathrm{C}(13)-\mathrm{C}(14)$ & $-178.3(11)$ \\
\hline $\mathrm{C}(9)-\mathrm{C}(8)-\mathrm{C}(13)-\mathrm{C}(12)$ & $-2.2(15)$ \\
\hline $\mathrm{C}(7)-\mathrm{C}(8)-\mathrm{C}(13)-\mathrm{C}(12)$ & $174.6(9)$ \\
\hline $\mathrm{C}(9)-\mathrm{C}(8)-\mathrm{C}(13)-\mathrm{C}(14)$ & $178.5(10)$ \\
\hline $\mathrm{C}(7)-\mathrm{C}(8)-\mathrm{C}(13)-\mathrm{C}(14)$ & $-4.7(15)$ \\
\hline $\mathrm{Ce}(1)-\mathrm{O}(3)-\mathrm{C}(17)-\mathrm{O}(4)$ & $-3.8(9)$ \\
\hline $\mathrm{Ce}(1)-\mathrm{O}(3)-\mathrm{C}(17)-\mathrm{C}(18)$ & $175.4(7)$ \\
\hline $\mathrm{Ce}(1)-\mathrm{O}(4)-\mathrm{C}(17)-\mathrm{O}(3)$ & $3.9(9)$ \\
\hline $\mathrm{Ce}(1)-\mathrm{O}(4)-\mathrm{C}(17)-\mathrm{C}(18)$ & $-175.4(7)$ \\
\hline $\mathrm{O}(3)-\mathrm{C}(17)-\mathrm{C}(18)-\mathrm{C}(23)$ & $19.6(14)$ \\
\hline $\mathrm{O}(4)-\mathrm{C}(17)-\mathrm{C}(18)-\mathrm{C}(23)$ & $-161.1(9)$ \\
\hline $\mathrm{O}(3)-\mathrm{C}(17)-\mathrm{C}(18)-\mathrm{C}(19)$ & $-157.8(8)$ \\
\hline $\mathrm{O}(4)-\mathrm{C}(17)-\mathrm{C}(18)-\mathrm{C}(19)$ & $21.4(12)$ \\
\hline $\mathrm{C}(23)-\mathrm{C}(18)-\mathrm{C}(19)-\mathrm{C}(20)$ & $0.8(13)$ \\
\hline $\mathrm{C}(17)-\mathrm{C}(18)-\mathrm{C}(19)-\mathrm{C}(20)$ & $178.4(9)$ \\
\hline $\mathrm{C}(18)-\mathrm{C}(19)-\mathrm{C}(20)-\mathrm{C}(21)$ & $2.0(15)$ \\
\hline$C(19)-C(20)-C(21)-C(22)$ & $-2.6(15)$ \\
\hline $\mathrm{C}(20)-\mathrm{C}(21)-\mathrm{C}(22)-\mathrm{C}(23)$ & $0.4(14)$ \\
\hline$C(19)-C(18)-C(23)-C(22)$ & $-3.0(13)$ \\
\hline $\mathrm{C}(17)-\mathrm{C}(18)-\mathrm{C}(23)-\mathrm{C}(22)$ & $179.6(8)$ \\
\hline$C(19)-C(18)-C(23)-C(24)$ & $-179.6(8)$ \\
\hline $\mathrm{C}(17)-\mathrm{C}(18)-\mathrm{C}(23)-\mathrm{C}(24)$ & $2.9(14)$ \\
\hline $\mathrm{C}(21)-\mathrm{C}(22)-\mathrm{C}(23)-\mathrm{C}(18)$ & $2.4(13)$ \\
\hline $\mathrm{C}(21)-\mathrm{C}(22)-\mathrm{C}(23)-\mathrm{C}(24)$ & $179.4(8)$ \\
\hline $\mathrm{C}(18)-\mathrm{C}(23)-\mathrm{C}(24)-\mathrm{C}(29)$ & $90.9(12)$ \\
\hline $\mathrm{C}(22)-\mathrm{C}(23)-\mathrm{C}(24)-\mathrm{C}(29)$ & $-86.0(11)$ \\
\hline
\end{tabular}




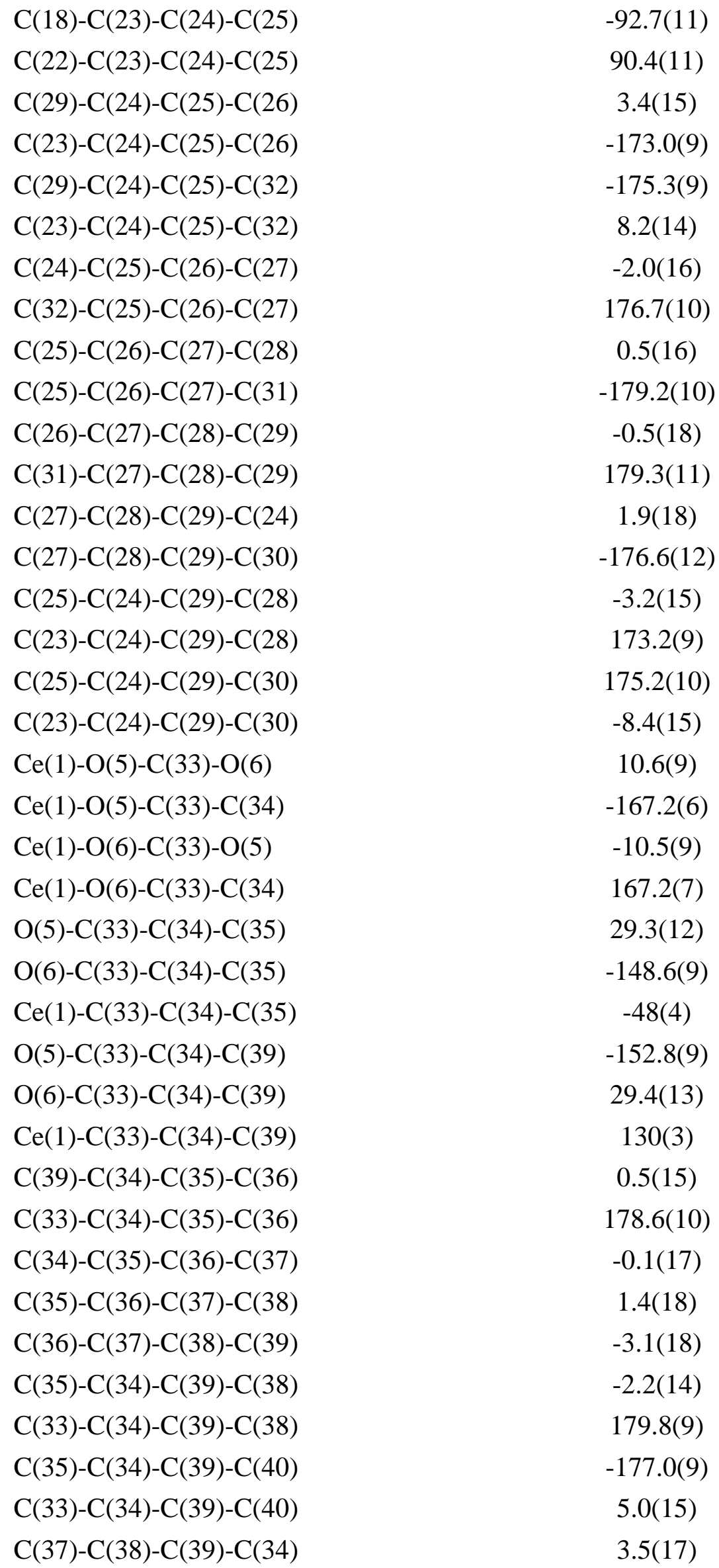




$\begin{array}{lc}\mathrm{C}(37)-\mathrm{C}(38)-\mathrm{C}(39)-\mathrm{C}(40) & 178.8(10) \\ \mathrm{C}(34)-\mathrm{C}(39)-\mathrm{C}(40)-\mathrm{C}(45) & 87.1(12) \\ \mathrm{C}(38)-\mathrm{C}(39)-\mathrm{C}(40)-\mathrm{C}(45) & -87.8(12) \\ \mathrm{C}(34)-\mathrm{C}(39)-\mathrm{C}(40)-\mathrm{C}(41) & -93.0(12) \\ \mathrm{C}(38)-\mathrm{C}(39)-\mathrm{C}(40)-\mathrm{C}(41) & 92.1(12) \\ \mathrm{C}(45)-\mathrm{C}(40)-\mathrm{C}(41)-\mathrm{C}(42) & 3.0(15) \\ \mathrm{C}(39)-\mathrm{C}(40)-\mathrm{C}(41)-\mathrm{C}(42) & -177.0(8) \\ \mathrm{C}(45)-\mathrm{C}(40)-\mathrm{C}(41)-\mathrm{C}(47) & -179.6(9) \\ \mathrm{C}(39)-\mathrm{C}(40)-\mathrm{C}(41)-\mathrm{C}(47) & 0.5(15) \\ \mathrm{C}(40)-\mathrm{C}(41)-\mathrm{C}(42)-\mathrm{C}(43) & -4.4(16) \\ \mathrm{C}(47)-\mathrm{C}(41)-\mathrm{C}(42)-\mathrm{C}(43) & 178.2(10) \\ \mathrm{C}(41)-\mathrm{C}(42)-\mathrm{C}(43)-\mathrm{C}(44) & 4.6(16) \\ \mathrm{C}(41)-\mathrm{C}(42)-\mathrm{C}(43)-\mathrm{C}(48) & -175.8(10) \\ \mathrm{C}(42)-\mathrm{C}(43)-\mathrm{C}(44)-\mathrm{C}(45) & -3.4(15) \\ \mathrm{C}(48)-\mathrm{C}(43)-\mathrm{C}(44)-\mathrm{C}(45) & 177.0(9) \\ \mathrm{C}(41)-\mathrm{C}(40)-\mathrm{C}(45)-\mathrm{C}(44) & -1.9(14) \\ \mathrm{C}(39)-\mathrm{C}(40)-\mathrm{C}(45)-\mathrm{C}(44) & 178.0(8) \\ \mathrm{C}(41)-\mathrm{C}(40)-\mathrm{C}(45)-\mathrm{C}(46) & -179.7(9) \\ \mathrm{C}(39)-\mathrm{C}(40)-\mathrm{C}(45)-\mathrm{C}(46) & 0.3(14) \\ \mathrm{C}(43)-\mathrm{C}(44)-\mathrm{C}(45)-\mathrm{C}(40) & 2.2(14) \\ \mathrm{C}(43)-\mathrm{C}(44)-\mathrm{C}(45)-\mathrm{C}(46) & 180.0(9) \\ & \end{array}$

Symmetry transformations used to generate equivalent atoms: 
Table S12. Hydrogen bonds for complex 56 [ $\AA^{\circ}$ and $\left.{ }^{\circ}\right]$.

\begin{tabular}{lcccc}
\hline & & & & \\
$\mathrm{D}-\mathrm{H} \ldots \mathrm{A}$ & $\mathrm{d}(\mathrm{D}-\mathrm{H})$ & $\mathrm{d}(\mathrm{H} \ldots \mathrm{A})$ & $\mathrm{d}(\mathrm{D} \ldots \mathrm{A})$ & $<(\mathrm{DHA})$ \\
& & & & \\
\hline $\mathrm{C}(1)-\mathrm{H}(1) \ldots \mathrm{F}(9)$ & 0.95 & 2.54 & $3.199(2)$ & 127.0 \\
$\mathrm{C}(2)-\mathrm{H}(2) \ldots \mathrm{F}(2) \# 1$ & 0.95 & 2.58 & $3.471(2)$ & 155.8 \\
$\mathrm{C}(4)-\mathrm{H}(4) \ldots \mathrm{F}(18) \# 2$ & 0.95 & 2.42 & $3.274(2)$ & 148.9 \\
$\mathrm{C}(14)-\mathrm{H}(14) \ldots \mathrm{F}(14) \# 3$ & 0.95 & 2.55 & $3.442(2)$ & 155.7 \\
$\mathrm{C}(15)-\mathrm{H}(15) \ldots \mathrm{F}(12)$ & 0.95 & 2.45 & $3.394(2)$ & 172.3 \\
$\mathrm{C}(16)-\mathrm{H}(16) \ldots \mathrm{F}(16) \# 4$ & 1.00 & 2.48 & $3.327(2)$ & 141.7 \\
$\mathrm{C}(16)-\mathrm{H}(16) \ldots \mathrm{N}(2)$ & 1.00 & 2.69 & $3.242(2)$ & 115.1 \\
$\mathrm{C}(19)-\mathrm{H}(19) \ldots \mathrm{O}(1)$ & 1.00 & 2.50 & $3.029(2)$ & 112.5 \\
$\mathrm{C}(22)-\mathrm{H}(22) \ldots \mathrm{O}(2)$ & 1.00 & 2.24 & $2.881(2)$ & 120.9 \\
$\mathrm{O}(4)-\mathrm{H}(4 \mathrm{~A}) \ldots \mathrm{O}(3) \# 5$ & 0.84 & 1.81 & $2.6299(17)$ & 164.5 \\
$\mathrm{C}(25)-\mathrm{H}(25) \ldots \mathrm{F}(15) \# 5$ & 1.00 & 2.50 & $3.231(2)$ & 129.6 \\
$\mathrm{C}(25)-\mathrm{H}(25) \ldots \mathrm{F}(17) \# 5$ & 1.00 & 2.59 & $3.257(2)$ & 124.2 \\
& & & & \\
\hline
\end{tabular}

Symmetry transformations used to generate equivalent atoms:

$\# 1-\mathrm{x}+1,-\mathrm{y}+1,-\mathrm{z}+1 \quad \# 2-\mathrm{x}+1,-\mathrm{y},-\mathrm{z}+1 \quad \# 3-\mathrm{x}+1,-\mathrm{y}+1,-\mathrm{z}$

$\# 4 \mathrm{x}+1, \mathrm{y}, \mathrm{z} \quad \# 5 \mathrm{x}, \mathrm{y}+1, \mathrm{z}$ 


\section{References}

1. Malaestean, I. L.; Kutluca-Alıc1, M.; Ellern, A.; Leusen, J.; Schilder, H.; Speldrich, M.; Baca, S. G.; Kögerler, P.; Cryst. Growth Des. 2012, 12, 1593-1602.

2. De Bruin-Dickason, C. N.; Deacon, G. B.; Forsyth, C. M.; Hanf, S.; Heilmann, O.B.; Hinton, B. R. W.; Junk, P. C.; Somers, A. E.; Tan, Y.-Q.; Turner, D. R. Aust. J. Chem. 2017, 70, 478 .

3. Morton, C. M.; Zhu, Q.; Ripberger, H.; Troian-Gautier, L.; Toa, Z. S. D.; Knowles, R. R.; Alexanian, E. J. J. Am. Chem. Soc. 2019, 141, 13253-13260.

4. Korolev, D. N.; Bumagin, Nikolay. A. Tetrahedron Letters 2006, 47, 4225-4229. 